\title{
Determinants of microvascular function in individuals with and without type 2 diabetes
}

Citation for published version (APA):

Sörensen, B. M. (2018). Determinants of microvascular function in individuals with and without type 2 diabetes: a population-based approach. [Doctoral Thesis, Maastricht University]. Maastricht University. https://doi.org/10.26481/dis.20180131bms

Document status and date:

Published: 01/01/2018

DOI:

10.26481/dis.20180131bms

Document Version:

Publisher's PDF, also known as Version of record

\section{Please check the document version of this publication:}

- A submitted manuscript is the version of the article upon submission and before peer-review. There can be important differences between the submitted version and the official published version of record.

People interested in the research are advised to contact the author for the final version of the publication, or visit the DOI to the publisher's website.

- The final author version and the galley proof are versions of the publication after peer review.

- The final published version features the final layout of the paper including the volume, issue and page numbers.

Link to publication

\footnotetext{
General rights rights.

- You may freely distribute the URL identifying the publication in the public portal. please follow below link for the End User Agreement:

www.umlib.nl/taverne-license

Take down policy

If you believe that this document breaches copyright please contact us at:

repository@maastrichtuniversity.nl

providing details and we will investigate your claim.
}

Copyright and moral rights for the publications made accessible in the public portal are retained by the authors and/or other copyright owners and it is a condition of accessing publications that users recognise and abide by the legal requirements associated with these

- Users may download and print one copy of any publication from the public portal for the purpose of private study or research.

- You may not further distribute the material or use it for any profit-making activity or commercial gain

If the publication is distributed under the terms of Article $25 \mathrm{fa}$ of the Dutch Copyright Act, indicated by the "Taverne" license above, 
Determinants of microvascular function in individuals with and without type 2 diabetes:

a population-based approach 
(C) Ben Sörensen, Maastricht 2017

No part of this book may be reproduced or transmitted in any form or by any means, without prior permission in writing by the author, or when appropriate, by the publishers of the publications.

Paranimfen:

Dana Sörensen

Marnix van Agtmaal

Layout: Tiny Wouters

Printed by: Proefschriftenprinten.nl

Cover: Jean Scheijen, Sylvia van der Lubbe \& Ben Sörensen

ISBN: 978-94-92679-24-6 


\title{
Determinants of microvascular function in individuals with and without type 2 diabetes: \\ a population-based approach
}

\author{
PROEFSCHRIFT \\ ter verkrijging van de graad van doctor aan de Universiteit Maastricht, \\ op gezag van de Rector Magnificus, Prof. dr. Rianne M. Letschert, \\ volgens het besluit van het College van Decanen, \\ in het openbaar te verdedigen \\ op woensdag 31 januari 2018 om 14.00 uur
}

door

Ben Madhu Sörensen 


\section{Promotor}

Prof. dr. C.D.A. Stehouwer

\section{Copromotores}

Dr. A.J.H.M. Houben

Dr. M.T. Schram

\section{Beoordelingscommissie}

Prof. dr. H.A.J. Struijker-Boudier (voorzitter)

Dr. J.W.J. Beulens (VU medisch centrum, Amsterdam)

Prof. dr. H.J.G.M. Crijns

Prof. dr. M.K.C. Hesselink

Dr. R.G. IJzerman (VU medisch centrum, Amsterdam) 


\section{Contents}

Chapter $1 \quad$ General introduction 7

$\begin{array}{lll}\text { PART I } & \text { (Pre)diabetes and generalized microvascular dysfunction } & 21\end{array}$

Chapter $2 \quad$ Prediabetes and type 2 diabetes are associated with 23

generalized microvascular dysfunction: The Maastricht Study

Chapter 3 Hyperglycemia is the main mediator of prediabetes- and type $2 \quad 59$

diabetes-associated impairment of microvascular function:

The Maastricht Study

PART II (Modifiable) cardiovascular risk factors as determinants of microvascular function

Chapter $4 \quad$ Cardiovascular risk factors as determinants of retinal and skin microvascular function: The Maastricht Study

Chapter 5 Higher levels of daily physical activity are associated with better skin microvascular function in type 2 diabetes:

The Maastricht Study

Chapter 6 Summary and general discussion

Nederlandstalige samenvatting

Valorization addendum

Scientific output

Dankwoord

Curriculum Vitae 



\section{Chapter 1}

General introduction 



\section{General introduction}

Type 2 diabetes mellitus (T2D) is a chronic metabolic disease characterized by hyperglycemia and caused by defects in insulin action and/or deficits in insulin secretion. T2D is strongly associated with an adverse cardiovascular risk factor profile with obesity ${ }^{1}$, hypertension ${ }^{2}$, low-grade inflammation ${ }^{1}$, and dyslipidemia ${ }^{3}$. Importantly, cardiovascular risk not only affects individuals with diabetes, but also those without. Both an adverse cardiovascular risk profile and/or having T2D are associated with an increased risk of diseases of macrovascular origin (e.g. myocardial infarction, stroke, and peripheral arterial disease $)^{4}$, and diseases which are partly or wholly of microvascular origin (e.g. heart failure ${ }^{5}$, (lacunar) stroke ${ }^{6}$, depression ${ }^{7}$, cognitive decline $^{8}$, retinopathy ${ }^{9}$, chronic kidney disease ${ }^{10}$, and neuropathy $\left.{ }^{9}\right)$. Macrovascular and microvascular diseases impose a substantial burden on patients, their surroundings, and health care systems. Therefore, there is a need to understand their causes, in order to identify targets for treatment and prevention. Microvascular dysfunction has been shown to be associated with the aforementioned diseases of microvascular origin, and therefore may be an important underlying mechanism ${ }^{5-10}$. This dissertation will further focus on determinants of microvascular dysfunction in order to understand its pathophysiology better. Microvascular, as opposed to macrovascular research comprises a relatively new and growing field. This is predominantly due to recent technical advances which now enable accurate and direct measurement of microvascular function on a population-based level. In this chapter we describe definition, function, and assessment of the microcirculation. Hypotheses on major cardiovascular risk factors as determinants of microvascular (dys)function will be explained. Finally the aims and outline of this dissertation will be formulated.

\section{Microcirculation: definition, function, and assessment}

\section{Definition and function}

The endothelium is a monolayer of endothelial cells that line the interior surface of all blood vessels, both in the macrocirculation and microcirculation, and is important in blood vessel physiology. As the microvasculature covers $\sim 98 \%$ of the total vascular surface area, $\sim 98 \%$ of the endothelium is within the microcirculation ${ }^{11}$. The microcirculation can be defined anatomically as blood vessels smaller than 200-150 $\mu \mathrm{m}$ in diameter and comprises capillaries, venules, and arterioles ${ }^{12}$.

The microcirculation is essential for the regulation of body and tissue metabolism, and blood pressure ${ }^{13,14}$. First, the microcirculation regulates the distribution of nutrients, 
oxygen, and metabolites, primarily by vasodilation and vasoconstriction in order to match the metabolic demand of the surrounding tissue. Second, the microcirculation regulates overall peripheral resistance, which avoids large fluctuations of hydrostatic pressure and stabilizes pressure at the level of the capillaries optimal for capillary exchange ${ }^{15,16}$. Finally, the microvasculature and endothelium are involved in regulation of many other processes such as hemostatic balance, blood cell trafficking, innate and adaptive immunity, and vasomotor tone ${ }^{17}$. In normal conditions, local, regional, and systemic auto-regulatory mechanisms ensure adequate progress of these microcirculatory functions. Microvascular function is the result of the complex interrelation among structure and function of vessel wall components (matrix, smooth muscle cells, and endothelium), which are also closely related to metabolic and neurogenic influences ${ }^{12}$.

\section{Estimates of microvascular function used in this dissertation}

Several techniques can be applied to assess microvascular (endothelial) function at different sites of the body ${ }^{18,19}$. The techniques used to assess microvascular function applied in The Maastricht Study, the primary source of data in this dissertation, will be described below. In the studies of this dissertation we will focus on the (nitric oxide / endothelium-dependent) vasodilation capacity of the retinal arteriolar microcirculation and the skin microcirculation under stimulated conditions ${ }^{20,21}$. We specifically chose the retina and skin, as these are easily accessible sites enabling direct, non-invasive, and reproducible $^{22,23}$ assessment of microvascular (endothelial) function ${ }^{20,21}$. Retinal microvascular (endothelial) function was measured by flicker light-induced retinal arteriolar dilation and skin microvascular (endothelial) function was measured by heatinduced skin hyperemia.

Retinal arteriolar vasodilation to flicker light exposure was measured by the Dynamic Vessel Analyzer (Imedos, Jena, Germany), and is thought to be related to nutritive demands of activated retinal neurons ${ }^{24}$. During the flicker light stimulation period, the retinal arteriolar vessel diameter (expressed in measurement units, where 1 measurement unit equals $1 \mu \mathrm{m}$ of the Gullstrand ey $\mathrm{e}^{25}$ ) was likely to increase, predominantly as a consequence of endothelium-dependent vascular smooth muscle cell relaxation ${ }^{20}$. Heat-induced skin hyperemia was measured by laser-Doppler flowmetry (Perimed, Järfälla, Sweden), and is thought to be related to the skin thermoregulatory function, in order to dissipate heat ${ }^{16}$. During the heating phase the skin was locally heated to $44^{\circ} \mathrm{C}$, after which an increase in skin blood flow (measured as perfusion units, i.e. the product of the velocity and concentration of moving red blood cells ${ }^{26}$ ) was observed due to endothelium-dependent local microvascular vasodilation ${ }^{21}$. 
Impairments in stimulus-induced retinal arteriolar and skin microvascular vasodilation capacity can be seen as a reflection of microvascular endothelial dysfunction, as both responses are (partly) blunted by inhibition of $N^{G}$-monomethylL-arginine (L-NMMA); a non-selective inhibitor of nitric oxide synthase ${ }^{20,27,28}$. Nitric oxide synthase in endothelial cells is an enzyme catalyzing the production of nitric oxide from L-arginine. Nitric oxide is an important endothelium-derived cellular signaling molecule, which relaxes vascular smooth muscle cells and thereby acts as a potent vasodilator. However, this implies that impairments in retinal and skin microvascular vasodilation responses possibly also depend on vascular smooth muscle cell dysfunction ${ }^{29,30}$. In addition, these responses may also be caused by neuronal dysfunction, as intact retinal and skin nerve signaling is necessary to sense and conduct the flicker and heat stimulus, respectively ${ }^{21,31}$.

\section{Other microvascular measures assessed in The Maastricht Study}

In addition to the measurements used in this dissertation, other techniques to assess microvascular function applied in The Maastricht Study will only be described briefly; e.g. in the skin (by nailfold capillaroscopy), retina (by fundus photography), tongue (by glycocalyx assessment), and brain (by magnetic resonance imaging markers of cerebral small vessel disease) ${ }^{32}$. Microvascular responses in the skin can be studied during the basal state and/or after applying a stimulus, such as heating, ischemia, or systemic or local administration of endothelium-(in)dependent vasoactive agents such as sodium nitroprusside and acetylcholine. Lower responses generally reflect microvascular (endothelial) dysfunction ${ }^{21,33,34}$. In addition, microvascular morphology can be investigated via retinal microvascular calibers ${ }^{35}$ and/or tortuosity ${ }^{36}$, and skin capillary density ${ }^{37}$.

An indirect method to assess microvascular function is the measurement of plasma biomarkers, such as soluble intracellular adhesion molecule 1 (sICAM-1), soluble vascular adhesion molecule (sVCAM-1), von Willebrand factor (VWF), and soluble E-selectin. Increased levels of these markers are assumed to reflect procoagulant and prothrombotic activity (i.e. VWF) ${ }^{38,39}$ and endothelial permeability to leucocytes (i.e. sICAM-1, sVCAM-1, and E-selectin) ${ }^{38,39}$. Higher levels of these biomarkers are likely to reflect microvascular endothelial dysfunction, as they mainly originate from the microvascular endothelium ${ }^{38,40}$. Another alternative indirect measure of microvascular endothelial function is urinary albumin excretion ${ }^{38,41}$, as it is thought that urinary albumin excretion reflects generalized microvascular endothelial permeability ${ }^{42}$. 


\section{Is it likely that microvascular endothelial dysfunction is a feature of prediabetes and type 2 diabetes?}

It is well known that T2D is associated with an increased risk of cardiovascular disease, which can be partly explained by large-artery endothelial dysfunction. However, largeartery endothelial dysfunction is already present in prediabetes ${ }^{43-45}$, which suggests that the pathogenesis of macrovascular disease in individuals with T2D starts prior to the diagnosis of T2D (ticking clock hypothesis) ${ }^{46}$. This may also explain the increased risk of macrovascular disease in individuals with prediabetes ${ }^{47}$.

The ticking clock hypothesis was originally based on the observation that an adverse cardiovascular risk profile (including elevated total and low-density lipoprotein cholesterol levels, triglycerides levels, body mass index, blood pressure, and lower levels of high-density lipoprotein cholesterol) was already present years before the clinical diagnosis of $\mathrm{T}^{2} \mathrm{D}^{48}$. It is however unknown whether microvascular endothelial dysfunction, similar to the ticking clock phenomenon of macrovascular endothelial dysfunction, also occurs in prediabetes. If this is the case it may explain the increased risk of complications that are (partly) of microvascular origin, which can occur in prediabetes or early in the course of $\mathrm{T}_{2} \mathrm{D}^{49}$. In view of the above, we hypothesized that microvascular endothelial dysfunction is present in prediabetes and further deteriorates in T2D.

If microvascular endothelial dysfunction proves to be a feature of prediabetes (and further deteriorates in T2D), it is important to understand the underlying mechanisms. Several studies suggest that both metabolic and vascular risk factors are likely to be involved ${ }^{13,50-55}$. Key metabolic features of $T 2 \mathrm{D}$ are progressive insulin resistance and $\beta$-cell dysfunction, which can lead to hyperglycemia and abnormal insulin signaling, which further aggravate hyperglycemia. Hyperglycemia may impair microvascular function, via the formation of advanced glycation end products and reactive oxygen species. Both can quench endothelium-derived nitric oxide and directly inhibit nitric oxide synthase activity, which leads to impaired nitric oxide bioavailability ${ }^{52,56-60}$ and may thereby impair microvascular vasodilation. However, T2D typically is associated with an adverse cardiovascular risk profile consisting of low-grade inflammation ${ }^{1}$, dyslipidemia ${ }^{3}$, hypertension ${ }^{2}$, and arterial stiffness ${ }^{44}$. Importantly, there is evidence that each of these comorbid vascular risk factors is also linked to microvascular dysfunction, for instance through adverse effects on the insulin signaling pathway, impairment of nitric oxide bioavailability, abnormal regulation of vasomotor tone, and/or increased pulsatile flow $^{50,51,53-55}$, all of which may affect microvascular vasodilation.

While multiple studies ${ }^{13,50-55}$ have shown pathways through which hyperglycemia, insulin resistance, hypertension, dyslipidemia, arterial stiffness, and low-grade inflammation may impact microvascular function, it has not been investigated whether 
the effects of prediabetes and T2D on microvascular endothelial function are dependent on the degree of those metabolic and vascular risk factors. This is important, as it may help to unravel the etiology of (pre)diabetes-associated microvascular endothelial dysfunction. Microvascular endothelial dysfunction is thought to be involved in the pathophysiology of numerous common diseases such as heart failure, (lacunar) stroke, depression, cognitive decline, retinopathy, chronic kidney disease, and neuropathy ${ }^{5-10,42,61}$. The ultimate goal is to find targets for strategies to prevent or reduce these common diseases, which frequently occur in individuals with $T 2 D^{62}$.

\section{Is it likely that microvascular endothelial dysfunction is affected by cardiovascular risk factors?}

Cardiovascular risk factors are associated with, and thus determinants of macrovascular diseases $^{63-65}$. The pathogenesis of macrovascular diseases involves large-artery endothelial dysfunction ${ }^{4}$, atherosclerosis ${ }^{43}$, and arterial stiffening ${ }^{44}$. However, due to endothelial cell heterogeneity (i.e. endothelial cells differ remarkably in function and structure depending on their localization), this does not necessarily imply that microvascular endothelial function is affected similarly ${ }^{66,67}$.

The pathophysiology of common diseases such as heart failure, (lacunar) stroke, depression, cognitive decline, retinopathy, chronic kidney disease, and neuropathy is thought to involve microvascular endothelial dysfunction ${ }^{5-10,42,61}$. As these diseases, which are (in part) of microvascular origin, affect both individuals with and without T2D, it is important to elucidate determinants of microvascular endothelial function in the general population.

Several small (mostly intervention) studies ${ }^{55,68-74}$ have investigated major cardiovascular risk factors as determinants of microvascular function. These cardiovascular risk factors included (directly) modifiable factors (e.g. current smoking, low levels of physical activity, high levels of sedentary time, hypertension, dyslipidemia, hyperglycemia, and obesity) and unmodifiable factors (e.g. aging and male sex). However, these studies ${ }^{55,68-74}$ were conducted with small numbers of highly selected individuals. For example, study populations were comprised of lean versus obese subjects with a body mass index of $21 \mathrm{~kg} / \mathrm{m}^{2}$ versus $39 \mathrm{~kg} / \mathrm{m}^{2}{ }^{68}$, or compared highly trained professional football players with untrained individuals ${ }^{74}$, which is not representative for habitual physical activity. Importantly and additionally, these studies were insufficiently adjusted for potential confounders. Both the insufficient adjustment and the highly selected study populations limit the translation of these findings to the general population.

Many cardiovascular risk factors of macrovascular diseases are also associated with microvascular diseases ${ }^{49,75-77}$, we therefore hypothesized that aging, male sex, 
hypertension, dyslipidemia, hyperglycemia, higher waist circumference, current smoking, low levels of physical activity, and high levels of sedentary time are also determinants of microvascular endothelial function.

\section{Cohort study used in this dissertation}

The results presented in this dissertation are based on data of The Maastricht Study ${ }^{32}$, an ongoing prospective population-based cohort study that focuses on the etiology, pathophysiology, complications, and comorbidities of T2D. A large sample of individuals aged 40-75 was recruited from the Maastricht and Heuvelland region with oversampling of individuals with T2D. The Maastricht Study is characterized by an extensive phenotyping approach, including an oral glucose tolerance test to determine prediabetes and T2D status (which is the gold standard), 24-h ambulatory blood pressure measurements, objectively measured levels of habitual physical activity and sedentary time, and extensive assessment of microvascular function. This approach provides unique opportunities to study associations of prediabetes, T2D, as well as major cardiovascular risk factors as determinants of microvascular function. For this dissertation, data were used from the first 3451 individuals who completed the baseline survey between November 2010 and September 2013.

\section{Aims and outline of this dissertation}

Microvascular endothelial dysfunction is an important underlying mechanism of common diseases which are (partly) of microvascular origin, such as heart failure, (lacunar) stroke, depression, cognitive decline, retinopathy, chronic kidney disease, and neuropathy ${ }^{5-10,42,61}$. Therefore, it is important to unravel determinants of microvascular (dys)function in the general population and specifically in individuals with T2D (who are at increased risk of developing these diseases), as it may highlight targets for the prevention of microvascular diseases. In view of the above, the general aims of this dissertation were to investigate, in a population-based setting, associations of prediabetes, T2D, and (modifiable) cardiovascular risk factors with retinal arteriolar and skin microvascular endothelial function (Figure 1.1). In addition, we investigated whether (pre)diabetes-associated microvascular dysfunction was potentially attributable to hyperglycemia, insulin resistance, hypertension, dyslipidemia, arterial stiffness, and low-grade inflammation. Strengths of the studies in this dissertation include the epidemiological approach; the size of the study population; the population-based 
design; the extensive phenotyping approach of individuals, which enabled the adjustment for extensive series of potential confounders, in order to reduce the risk of residual confounding and thereby allow the investigation of relatively unbiased associations; and the use of two independent methods to directly assess microvascular endothelial function in different microvascular beds.

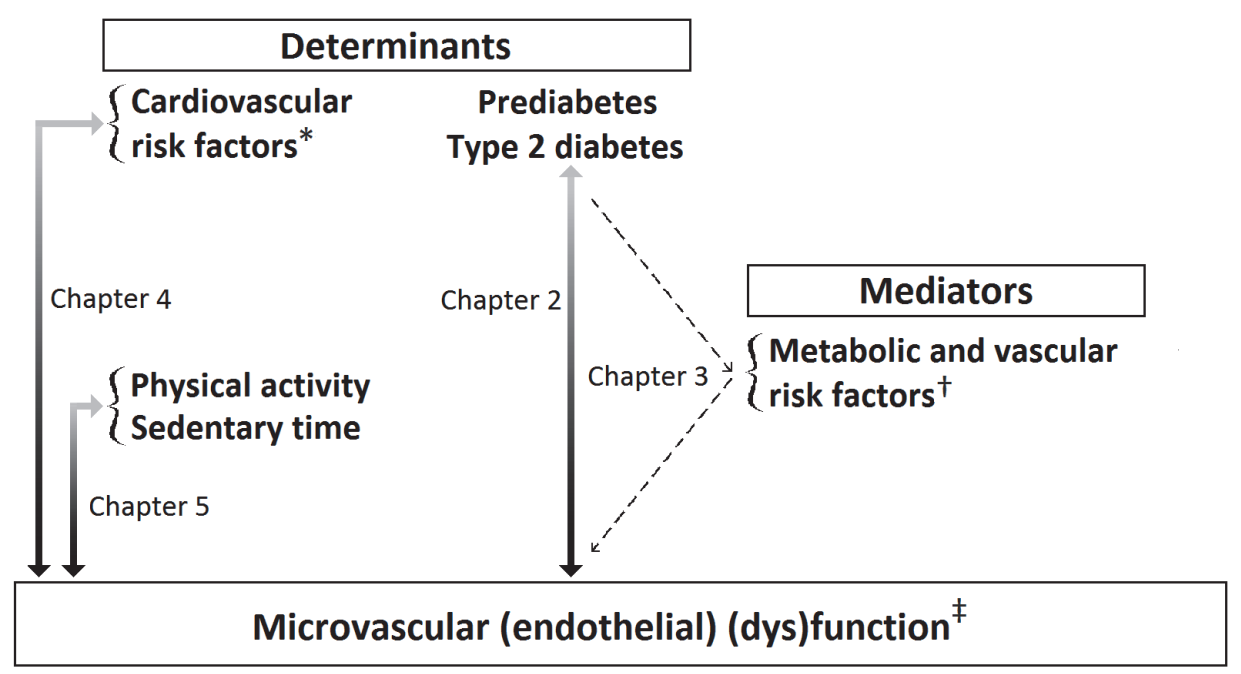

Figure 1.1 Schematic representation of the associations (bold arrows) and mediating effects (dashed arrows) investigated in the present dissertation.

* Cardiovascular risk factors investigated as proposed determinants of microvascular (endothelial) function included aging, male sex, hypertension, dyslipidemia, hyperglycemia (including prediabetes and type 2 diabetes), higher waist circumference, and current smoking. + Metabolic and vascular risk factors investigated as proposed mediators of (pre)diabetesassociated microvascular (endothelial) dysfunction included hyperglycemia, insulin resistance, arterial stiffness, hypertension, dyslipidemia, and low-grade inflammation. ${ }^{\ddagger}$ Microvascular (endothelial) function was assessed in the retina and skin as flicker light-induced retinal arteriolar dilation and heat-induced skin hyperemia, respectively. The bold black arrows indicate directions of the associations as hypothesized in this dissertation. Note that the direction of the associations of hyperglycemia (including prediabetes and type 2 diabetes) ${ }^{54}$, low levels of physical activity ${ }^{54}$, and some of the other cardiovascular risk factors (such as hypertension ${ }^{78}$ and dyslipidemia $^{79}$ ) with microvascular (endothelial) function can hypothetically also be reversed (bold gray arrows). 
In chapter 2 we investigated whether microvascular endothelial dysfunction already occurs in prediabetes. We tested the hypothesis that microvascular dysfunction is already present in prediabetes, and further deteriorates in T2D, by investigating the associations of prediabetes, T2D, and continuous plasma levels of glycemia with retinal arteriolar and skin microvascular endothelial function.

In chapter 3 we investigated, using a mediation analysis approach, whether, and to what extent, prediabetes- and T2D-associated retinal arteriolar and skin microvascular endothelial dysfunction were attributable to hyperglycemia, insulin resistance, blood pressure, lipid profile, arterial stiffness, and low-grade inflammation.

In chapter 4 we investigated whether determinants of large-artery endothelial dysfunction such as aging, male sex, hypertension, dyslipidemia, hyperglycemia, higher waist circumference, and current smoking are also determinants of retinal and skin microvascular endothelial dysfunction.

Low levels of physical activity and high levels of sedentary time are both directly modifiable cardiovascular risk factors. In chapter 5 we examined whether objectively measured levels of habitual physical activity and sedentary time were associated with skin microvascular and retinal arteriolar endothelial function.

Finally, chapter 6 summarizes and discusses the results of the studies in this dissertation and puts the findings into a broader perspective. In addition, methodological limitations will be considered as well as implications for future research. 


\section{References}

1. Hajer GR, van Haeften TW and Visseren FL. Adipose tissue dysfunction in obesity, diabetes, and vascular diseases. Eur Heart J. 2008;29:2959-71.

2. Lonati C, Morganti A, Comarella L, Mancia G, Zanchetti A and Group IS. Prevalence of type 2 diabetes among patients with hypertension under the care of 30 Italian clinics of hypertension: results of the (Iper)tensione and (dia)bete study. J Hypertens. 2008;26:1801-8.

3. Anderson RA, Evans ML, Ellis GR, Graham J, Morris K, Jackson SK, Lewis MJ, Rees A and Frenneaux MP. The relationships between post-prandial lipaemia, endothelial function and oxidative stress in healthy individuals and patients with type 2 diabetes. Atherosclerosis. 2001;154:475-83.

4. van Sloten TT, Henry RM, Dekker JM, Nijpels G, Unger T, Schram MT and Stehouwer CD. Endothelial dysfunction plays a key role in increasing cardiovascular risk in type 2 diabetes: the Hoorn study. Hypertension. 2014;64:1299-305.

5. Lee JF, Barrett-O'Keefe Z, Garten RS, Nelson AD, Ryan JJ, Nativi JN, Richardson RS and Wray DW. Evidence of microvascular dysfunction in heart failure with preserved ejection fraction. Heart. 2016;102:278-84.

6. Knottnerus IL, Ten Cate H, Lodder J, Kessels F and van Oostenbrugge RJ. Endothelial dysfunction in lacunar stroke: a systematic review. Cerebrovasc Dis. 2009;27:519-26.

7. Santos M, Xekardaki A, Kovari E, Gold G, Bouras C and Giannakopoulos P. Microvascular pathology in late-life depression. J Neurol Sci. 2012;322:46-9.

8. De Silva TM and Faraci FM. Microvascular Dysfunction and Cognitive Impairment. Cell Mol Neurobiol. 2016;36:241-58.

9. Gupta A and Bhatnagar S. Vasoregression: A Shared Vascular Pathology Underlying Macrovascular And Microvascular Pathologies? OMICS. 2015;19:733-53.

10. Zafrani L and Ince C. Microcirculation in Acute and Chronic Kidney Diseases. Am J Kidney Dis. 2015;66:1083-94.

11. Wolinsky H. A proposal linking clearance of circulating lipoproteins to tissue metabolic activity as a basis for understanding atherogenesis. Circ Res. 1980;47:301-11.

12. Houben AJ, Martens RJ and Stehouwer CD. Assessing microvascular function in humans from a chronic disease perspective. J Am Soc Nephrol. 2017; in press.

13. De Boer MP, Meijer RI, Wijnstok NJ, Jonk AM, Houben AJ, Stehouwer CD, Smulders YM, Eringa EC and Serne $\mathrm{EH}$. Microvascular dysfunction: a potential mechanism in the pathogenesis of obesity-associated insulin resistance and hypertension. Microcirculation. 2012;19:5-18.

14. Houben AJ, Eringa EC, Jonk AM, Serne EH, Smulders YM and Stehouwer CD. Perivascular Fat and the Microcirculation: Relevance to Insulin Resistance, Diabetes, and Cardiovascular Disease. Curr Cardiovasc Risk Rep. 2012;6:80-90.

15. Levy BI, Ambrosio G, Pries AR and Struijker-Boudier HA. Microcirculation in hypertension: a new target for treatment? Circulation. 2001;104:735-40.

16. Roustit $\mathrm{M}$ and Cracowski JL. Assessment of endothelial and neurovascular function in human skin microcirculation. Trends Pharmacol Sci. 2013;34:373-84.

17. Aird WC. Phenotypic heterogeneity of the endothelium: I. Structure, function, and mechanisms. Circ Res. 2007;100:158-73.

18. Knotzer $\mathrm{H}$ and Hasibeder WR. Microcirculatory function monitoring at the bedside - a view from the intensive care. Physiol Meas. 2007;28:R65-86.

19. Rizzoni D, Aalkjaer C, De Ciuceis C, Porteri E, Rossini C, Rosei CA, Sarkar A and Rosei EA. How to assess microvascular structure in humans. High Blood Press Cardiovasc Prev. 2011;18:169-77.

20. Dorner GT, Garhofer G, Kiss B, Polska E, Polak K, Riva CE and Schmetterer L. Nitric oxide regulates retinal vascular tone in humans. Am J Physiol Heart Circ Physiol. 2003;285:H631-6.

21. Minson CT, Berry LT and Joyner MJ. Nitric oxide and neurally mediated regulation of skin blood flow during local heating. J Appl Physiol (1985). 2001;91:1619-26.

22. Nguyen TT, Kreis AJ, Kawasaki R, Wang JJ, Seifert BU, Vilser W, Nagel E and Wong TY. Reproducibility of the retinal vascular response to flicker light in Asians. Curr Eye Res. 2009;34:1082-8. 
23. Agarwal SC, Allen J, Murray A and Purcell IF. Comparative reproducibility of dermal microvascular blood flow changes in response to acetylcholine iontophoresis, hyperthermia and reactive hyperaemia. Physiol Meas. 2010;31:1-11.

24. Nagel $E$ and Vilser W. Flicker observation light induces diameter response in retinal arterioles: a clinical methodological study. Br J Ophthalmol. 2004;88:54-6.

25. Nagel E, Vilser W, Fink A and Riemer T. Variance of retinal vessel diameter response to flicker light. A methodical clinical study. Ophthalmologe. 2006;103:114-9.

26. Braverman IM, Schechner JS, Silverman DG and Keh-Yen A. Topographic mapping of the cutaneous microcirculation using two outputs of laser-Doppler flowmetry: flux and the concentration of moving blood cells. Microvasc Res. 1992;44:33-48.

27. Choi PJ, Brunt VE, Fujii N and Minson CT. New approach to measure cutaneous microvascular function: an improved test of NO-mediated vasodilation by thermal hyperemia. J Appl Physiol (1985). 2014;117:277-83.

28. Kellogg DL, Jr., Liu Y, Kosiba IF and O'Donnell D. Role of nitric oxide in the vascular effects of local warming of the skin in humans. J App/ Physiol (1985). 1999;86:1185-90.

29. Montero D, Pierce GL, Stehouwer CD, Padilla J and Thijssen DH. The impact of age on vascular smooth muscle function in humans. J Hypertens. 2015;33:445-53.

30. Lacolley P, Regnault V, Nicoletti A, Li Z and Michel JB. The vascular smooth muscle cell in arterial pathology: a cell that can take on multiple roles. Cardiovasc Res. 2012;95:194-204.

31. Falsini B, Riva CE and Logean E. Flicker-evoked changes in human optic nerve blood flow: relationship with retinal neural activity. Invest Ophthalmol Vis Sci. 2002;43:2309-16.

32. Schram MT, Sep SJ, van der Kallen CJ, Dagnelie PC, Koster A, Schaper NC, Henry RM and Stehouwer CD. The Maastricht Study: an extensive phenotyping study on determinants of type 2 diabetes, its complications and its comorbidities. Eur J Epidemiol. 2014;29:439-51.

33. Jonk AM, Houben AJ, de Jongh RT, Serne EH, Schaper NC and Stehouwer CD. Microvascular dysfunction in obesity: a potential mechanism in the pathogenesis of obesity-associated insulin resistance and hypertension. Physiology. 2007;22:252-60.

34. Serne EH, Gans RO, ter Maaten JC, Tangelder GJ, Donker AJ and Stehouwer CD. Impaired skin capillary recruitment in essential hypertension is caused by both functional and structural capillary rarefaction. Hypertension. 2001;38:238-42.

35. Guedri H, Ben Abdallah M, Echouchene F and Belmabrouk H. Novel Computerized Method for Measurement of Retinal Vessel Diameters. Biomedicines. 2017;5.

36. Cheung CY, Zheng Y, Hsu W, Lee ML, Lau QP, Mitchell P, Wang JJ, Klein R and Wong TY. Retinal vascular tortuosity, blood pressure, and cardiovascular risk factors. Ophthalmology. 2011;118:812-8.

37. Gronenschild EH, Muris DM, Schram MT, Karaca U, Stehouwer CD and Houben AJ. Semi-automatic assessment of skin capillary density: proof of principle and validation. Microvasc Res. 2013;90:192-8.

38. Stehouwer CD. Is measurement of endothelial dysfunction clinically useful? Eur J Clin Invest. 1999;29:459-61.

39. Stehouwer CD. Endothelial dysfunction in diabetic nephropathy: state of the art and potential significance for non-diabetic renal disease. Nephrol Dial Transplant. 2004;19:778-81.

40. Szmitko PE, Wang CH, Weisel RD, de Almeida JR, Anderson TJ and Verma S. New markers of inflammation and endothelial cell activation: Part I. Circulation. 2003;108:1917-23.

41. Deckert T, Feldt-Rasmussen B, Borch-Johnsen K, Jensen T and Kofoed-Enevoldsen A. Albuminuria reflects widespread vascular damage. The Steno hypothesis. Diabetologia. 1989;32:219-26.

42. Seliger SL, Salimi S, Pierre V, Giffuni J, Katzel L and Parsa A. Microvascular endothelial dysfunction is associated with albuminuria and CKD in older adults. BMC Nephrol. 2016;17:82.

43. Mostaza JM, Lahoz C, Salinero-Fort MA, de Burgos-Lunar C, Laguna F, Estirado E, Garcia-Iglesias F, Gonzalez-Alegre T, Cornejo-Del-Rio V, Sabin C, Lopez S and Group S-. Carotid atherosclerosis severity in relation to glycemic status: a cross-sectional population study. Atherosclerosis. 2015;242:377-82.

44. Schram MT, Henry RM, van Dijk RA, Kostense PJ, Dekker JM, Nijpels G, Heine RJ, Bouter LM, Westerhof N and Stehouwer CD. Increased central artery stiffness in impaired glucose metabolism and type 2 diabetes: the Hoorn Study. Hypertension. 2004;43:176-81.

45. Su Y, Liu XM, Sun YM, Wang YY, Luan Y and Wu Y. Endothelial dysfunction in impaired fasting glycemia, impaired glucose tolerance, and type 2 diabetes mellitus. Am J Cardiol. 2008;102:497-8. 
46. Wong MS, Gu K, Heng D, Chew SK, Chew LS and Tai ES. The Singapore impaired glucose tolerance followup study: does the ticking clock go backward as well as forward? Diabetes Care. 2003;26:3024-30.

47. Ford ES, Zhao G and Li C. Pre-diabetes and the risk for cardiovascular disease: a systematic review of the evidence. J Am Coll Cardiol. 2010;55:1310-7.

48. Haffner SM, Stern MP, Hazuda HP, Mitchell BD and Patterson JK. Cardiovascular risk factors in confirmed prediabetic individuals. Does the clock for coronary heart disease start ticking before the onset of clinical diabetes? JAMA. 1990;263:2893-8.

49. Buysschaert M, Medina JL, Bergman M, Shah A and Lonier J. Prediabetes and associated disorders. Endocrine. 2015;48:371-93.

50. Mitchell GF, Vita JA, Larson MG, Parise H, Keyes MJ, Warner E, Vasan RS, Levy D and Benjamin EJ. Crosssectional relations of peripheral microvascular function, cardiovascular disease risk factors, and aortic stiffness: the Framingham Heart Study. Circulation. 2005;112:3722-8.

51. Shore AC and Tooke JE. Microvascular function in human essential hypertension. J Hypertens. 1994;12:717-28.

52. Serne EH, Stehouwer CD, ter Maaten JC, ter Wee PM, Rauwerda JA, Donker AJ and Gans RO. Microvascular function relates to insulin sensitivity and blood pressure in normal subjects. Circulation. 1999;99:896-902.

53. Cheng C and Daskalakis C. Association of Adipokines with Insulin Resistance, Microvascular Dysfunction, and Endothelial Dysfunction in Healthy Young Adults. Mediators Inflamm. 2015;2015:594039.

54. Muris DM, Houben AJ, Schram MT and Stehouwer CD. Microvascular dysfunction: an emerging pathway in the pathogenesis of obesity-related insulin resistance. Rev Endocr Metab Disord. 2013;14:29-38.

55. Reimann $\mathrm{M}$, Weiss $\mathrm{N}$ and Ziemssen T. Different responses of the retinal and cutaneous microcirculation to transient dysmetabolic conditions. Atheroscler Suppl. 2015;18:1-7.

56. Kim JA, Montagnani M, Koh KK and Quon MJ. Reciprocal relationships between insulin resistance and endothelial dysfunction: molecular and pathophysiological mechanisms. Circulation. 2006;113:1888-904.

57. Soro-Paavonen A, Zhang WZ, Venardos K, Coughlan MT, Harris E, Tong DC, Brasacchio D, Paavonen K, Chin-Dusting J, Cooper ME, Kaye D, Thomas MC and Forbes JM. Advanced glycation end-products induce vascular dysfunction via resistance to nitric oxide and suppression of endothelial nitric oxide synthase. J Hypertens. 2010;28:780-8.

58. Clark MG. Impaired microvascular perfusion: a consequence of vascular dysfunction and a potential cause of insulin resistance in muscle. Am J Physiol Endocrinol Metab. 2008;295:E732-50.

59. Verbeke $P$, Perichon $M$, Friguet $B$ and Bakala H. Inhibition of nitric oxide synthase activity by early and advanced glycation end products in cultured rabbit proximal tubular epithelial cells. Biochim Biophys Acta. 2000;1502:481-94.

60. Vallejo S, Angulo J, Peiro C, Cercas E, Sanchez-Ferrer A, Nevado J, Llergo JL, Rodriguez-Manas L and Sanchez-Ferrer CF. Treatment with acarbose may improve endothelial dysfunction in streptozotocininduced diabetic rats. J Cardiovasc Pharmacol. 2000;36:255-62.

61. Malecki MT, Osmenda G, Walus-Miarka M, Skupien J, Cyganek K, Mirkiewicz-Sieradzka B, damek-Guzik TA, Guzik TJ and Sieradzki J. Retinopathy in type 2 diabetes mellitus is associated with increased intimamedia thickness and endothelial dysfunction. Eur J Clin Invest. 2008;38:925-30.

62. Stratton IM, Adler Al, Neil HA, Matthews DR, Manley SE, Cull CA, Hadden D, Turner RC and Holman RR. Association of glycaemia with macrovascular and microvascular complications of type 2 diabetes (UKPDS 35): prospective observational study. BMJ. 2000;321:405-12.

63. Tsao CW and Vasan RS. Cohort Profile: The Framingham Heart Study (FHS): overview of milestones in cardiovascular epidemiology. Int J Epidemiol. 2015;44:1800-13.

64. Kannel WB, Dawber TR, Kagan A, Revotskie N and Stokes J, 3rd. Factors of risk in the development of coronary heart disease: six year follow-up experience. The Framingham Study. Ann Intern Med. 1961;55:33-50.

65. Singer DE, Nathan DM, Anderson KM, Wilson PW and Evans JC. Association of HbA1c with prevalent cardiovascular disease in the original cohort of the Framingham Heart Study. Diabetes. 1992;41:202-8.

66. Aird WC. Endothelial cell heterogeneity. Cold Spring Harb Perspect Med. 2012;2:a006429.

67. Aird WC. Mechanisms of endothelial cell heterogeneity in health and disease. Circ Res. 2006;98:159-62. 
68. de Jongh RT, Serne EH, IJzerman RG, de Vries G and Stehouwer CD. Impaired microvascular function in obesity: implications for obesity-associated microangiopathy, hypertension, and insulin resistance. Circulation. 2004;109:2529-35.

69. Nagel E, Vilser W and Lanzl I. Age, blood pressure, and vessel diameter as factors influencing the arterial retinal flicker response. Invest Ophthalmol Vis Sci. 2004;45:1486-92.

70. IJzerman RG, Serne EH, van Weissenbruch MM, de Jongh RT and Stehouwer CD. Cigarette smoking is associated with an acute impairment of microvascular function in humans. Clin Sci. 2003;104: 247-52.

71. IJzerman RG, de Jongh RT, Beijk MA, van Weissenbruch MM, Delemarre-van de Waal HA, Serne EH and Stehouwer CD. Individuals at increased coronary heart disease risk are characterized by an impaired microvascular function in skin. Eur J Clin Invest. 2003;33:536-42.

72. Irving RJ, Walker BR, Noon JP, Watt GC, Webb DJ and Shore AC. Microvascular correlates of blood pressure, plasma glucose, and insulin resistance in health. Cardiovasc Res. 2002;53:271-6.

73. Caballero AE, Arora S, Saouaf R, Lim SC, Smakowski P, Park JY, King GL, LoGerfo FW, Horton ES and Veves A. Microvascular and macrovascular reactivity is reduced in subjects at risk for type 2 diabetes. Diabetes. 1999;48:1856-62.

74. Roche DM, Rowland TW, Garrard M, Marwood S and Unnithan VB. Skin microvascular reactivity in trained adolescents. Eur J Appl Physiol. 2010;108:1201-8.

75. Fraser-Bell S, Symes R and Vaze A. Hypertensive eye disease: a review. Clin Exp Ophthalmol. 2017;45: 45-53.

76. Wickman $\mathrm{C}$ and Kramer H. Obesity and kidney disease: potential mechanisms. Semin Nephrol. 2013;33:14-22.

77. Parekh A, Smeeth D, Milner Y and Thure S. The Role of Lipid Biomarkers in Major Depression. Healthcare. 2017;5

78. Karaca U, Schram MT, Houben AJ, Muris DM and Stehouwer CD. Microvascular dysfunction as a link between obesity, insulin resistance and hypertension. Diabetes Res Clin Pract. 2014;103:382-7.

79. Olivecrona G. Role of lipoprotein lipase in lipid metabolism. Curr Opin Lipidol. 2016;27:233-41. 
Part

(Pre)diabetes and generalized microvascular dysfunction 



\section{Chapter}

Prediabetes and type 2 diabetes are associated with generalized microvascular dysfunction:

The Maastricht Study

Ben M. Sörensen, Alfons J.H.M. Houben, Tos T.J.M. Berendschot, Jan S.A.G. Schouten, Abraham A. Kroon, Carla J.H. van der Kallen, Ronald M.A. Henry, Annemarie Koster, Simone J.S. Sep, Pieter C. Dagnelie, Nicolaas C. Schaper, Miranda T. Schram, Coen D.A. Stehouwer

Circulation. 2016;134:1339-52 


\section{Abstract}

\section{Objective}

Type 2 diabetes (T2D) is associated with an increased risk of cardiovascular disease. This can be partly explained by large-artery dysfunction, which already occurs in prediabetes ('ticking clock hypothesis'). Whether a similar phenomenon also applies to microvascular dysfunction is not known. We therefore tested the hypothesis that microvascular dysfunction is already present in prediabetes and is more severe in T2D. To do so, we investigated the associations of prediabetes, $\mathrm{T} 2 \mathrm{D}$, and measures of hyperglycemia with microvascular function measured as flicker lightinduced retinal arteriolar dilation and heat-induced skin hyperemia.

\section{Methods}

In The Maastricht Study, a T2D-enriched population-based cohort study ( $n=2213,51 \%$ men, aged $59.7 \pm 8.2$ years), we determined flicker light-induced retinal arteriolar \%-dilation (Dynamic Vessel Analyzer), heat-induced skin \%-hyperemia (laser-Doppler flowmetry), and glucose metabolism status (OGTT; normal glucose metabolism (NGM) ( $n=1269)$, prediabetes $(n=335)$, or T2D $(n=609)$ ). Differences were assessed with multivariable regression analyses adjusted for age, sex, body mass index, smoking, physical activity, systolic blood pressure, lipid profile, retinopathy, estimated glomerular filtration rate, (micro)albuminuria, the use of lipid-modifying and/or blood pressurelowering medication, and history of cardiovascular disease.

\section{Results}

Retinal arteriolar \%-dilation was (mean \pm SD) $3.4 \pm 2.8$ in NGM, 3.0 \pm 2.7 in prediabetes, and $2.3 \pm 2.6$ in T2D. Adjusted analyses showed a lower arteriolar \%-dilation in prediabetes $(B=-0.20$, $95 \% \mathrm{Cl}(-0.56 ; 0.15))$, with further deterioration in T2D $(B=-0.61,(-0.97 ;-0.25))$ versus NGM, $P$ for trend $=0.001$. Skin \%-hyperemia was (mean \pm SD) $1235 \pm 810$ in NGM, $1109 \pm 748$ in prediabetes, and $937 \pm 683$ in T2D. Adjusted analyses showed a lower \%-hyperemia in prediabetes $(B=-46,(-163 ; 72))$, with further deterioration in T2D $(B=-184,(-297 ;-71))$ versus NGM, $P$ for trend=0.001. In addition, higher $\mathrm{HbA1C}$ and fasting plasma glucose (FPG) were associated with lower retinal arteriolar \%-dilation and skin \%-hyperemia in fully adjusted models (for HbA1c, standardized $B(s t B)=-0.10,(-0.15 ;-0.05), P<0.001$ and $s t B=-0.13, \quad(-0.19 ;-0.07), P<0.001$, respectively; for $F P G$, st $B=-0.09,(-0.15 ;-0.04), P<0.001$ and $s t B=-0.10,(-0.15 ;-0.04), P=0.002$, respectively).

\section{Conclusions}

Prediabetes, T2D, and measures of hyperglycemia are independently associated with impaired microvascular function in the retina and skin. These findings support the concept that microvascular dysfunction precedes and thus may contribute to T2D-associated cardiovascular disease and other complications which may in part have a microvascular origin, such as impaired cognition and heart failure. 


\section{Introduction}

The current worldwide epidemic of type 2 diabetes (T2D) ${ }^{1}$ implies an epidemic of its complications, both macrovascular (myocardial infarction, stroke, and peripheral arterial disease $)^{1-3}$ and microvascular. The latter not only comprises retinopathy and nephropathy ${ }^{3}$, but also complications that are partly of microvascular origin, notably neuropathy, heart failure ${ }^{4}$, stroke, depression, and cognitive dysfunction ${ }^{5}$.

The pathogenesis of diabetic macrovascular complications is thought to involve large-artery endothelial dysfunction ${ }^{2}$, atherosclerosis ${ }^{6}$, and arterial stiffening ${ }^{7}$. Such macrovascular dysfunction also occurs, although in less severe forms, in prediabetes ${ }^{6-8}$, suggesting that the pathogenesis of T2D-associated macrovascular disease starts prior to the diagnosis of T2D (ticking clock hypothesis ${ }^{9}$ ), which also explains the increased risk of macrovascular disease in individuals with prediabetes ${ }^{10}$.

Whether individuals with prediabetes in addition have microvascular dysfunction has not been studied systematically ${ }^{8,11-13}$. However, some individuals have microvascular complications at the time of diagnosis of T2D, and prediabetes has been associated not only with risk of retinopathy and nephropathy, but also with that of neuropathy, heart failure, stroke, and cognitive decline, which may, in part, have a microvascular origin ${ }^{5}$. Taken together, these data ${ }^{5,9,10}$ raise the possibility that microvascular, similar to macrovascular, dysfunction also may occur prior to the diagnosis of T2D.

In view of these considerations, we tested, in a population-based cohort study, the hypothesis that prediabetes, T2D, and measures of hyperglycemia are associated with microvascular function in the retina and skin, independently of potential confounders. We chose retina and skin as these are unique sites enabling direct and reproducible ${ }^{14,15}$ assessment of microvascular function, as measured by flicker light-induced retinal arteriolar dilation and heat-induced skin hyperemia ${ }^{16,17}$.

\section{Methods}

\section{Study population and design}

We used data from The Maastricht Study, an observational prospective populationbased cohort study. The rationale and methodology have been described previously ${ }^{18}$. In brief, the study focuses on the etiology, pathophysiology, complications, and comorbidities of T2D and is characterized by an extensive phenotyping approach. Eligible for participation were all individuals aged between 40 and 75 years and living in the southern part of the Netherlands. Participants were recruited through mass media campaigns and from the municipal registries and the regional Diabetes Patient Registry 
via mailings. Recruitment was stratified according to known T2D status, with an oversampling of individuals with T2D, for reasons of efficiency. The present report includes cross-sectional data from the first 3451 participants, who completed the baseline survey between November 2010 and September 2013. The examinations of each participant were performed within a time window of three months. The study has been approved by the institutional medical ethical committee (NL31329.068.10) and the Minister of Health, Welfare and Sports of The Netherlands (Permit 131088-105234-PG). All participants gave written informed consent. From the initial 3451 participants included, those with other types of diabetes than T2D were excluded $(n=41)$. Of the remaining 3410 participants, retinal arteriolar reactivity data were available in 2261 participants. The main reasons for missing data were logistical $(n=882)$, contraindications $(n=59)$, or insufficient measurement quality $(n=208)$. Covariates were missing in 48 participants. The retinal arteriolar reactivity study population thus consisted of 2213 participants. Heat-induced skin hyperemia data were available in 1647 of the 3410 participants. The reason for missing data were logistical ( $n=1650)$, technical $(n=24)$, or insufficient measurement quality $(n=89)$. Covariates were missing in 52 participants. The heat-induced skin hyperemia study population thus consisted of 1595 participants (Figure 2.1 shows the flow chart).

\section{Assessment of glucose metabolism status}

To assess glucose metabolism status, all participants (except those who used insulin) underwent a standardized 2-h 75 gram oral glucose tolerance test (OGTT) after an overnight fast. For safety reasons, participants with a fasting glucose level above $11.0 \mathrm{mmol} / \mathrm{l}$, as determined by a finger prick, did not undergo the OGTT. For these individuals fasting glucose level and information about diabetes medication use were used to assess glucose metabolism status. Glucose metabolism status was defined according to the World Health Organization 2006 criteria as normal glucose metabolism (NGM), impaired fasting glucose, impaired glucose tolerance (combined as prediabetes), or $\mathrm{T}^{\mathrm{D}} \mathrm{D}^{18}$.

\section{Assessment of microvascular function}

All participants were asked to refrain from smoking and drinking caffeine-containing beverages three hours before the measurement ${ }^{19}$. A light meal (breakfast and (or) lunch), low in fat content, was allowed at last 90 minutes prior to the start of the measurements. For retinal measurements pupils were dilated with $0.5 \%$ tropicamide and $2.5 \%$ phenylephrine at least 15 minutes prior to the start of the examination. Skin blood flow measurements were performed in a climate-controlled room at $24^{\circ} \mathrm{C}^{20}$. 
(Pre)diabetes and microvascular dysfunction

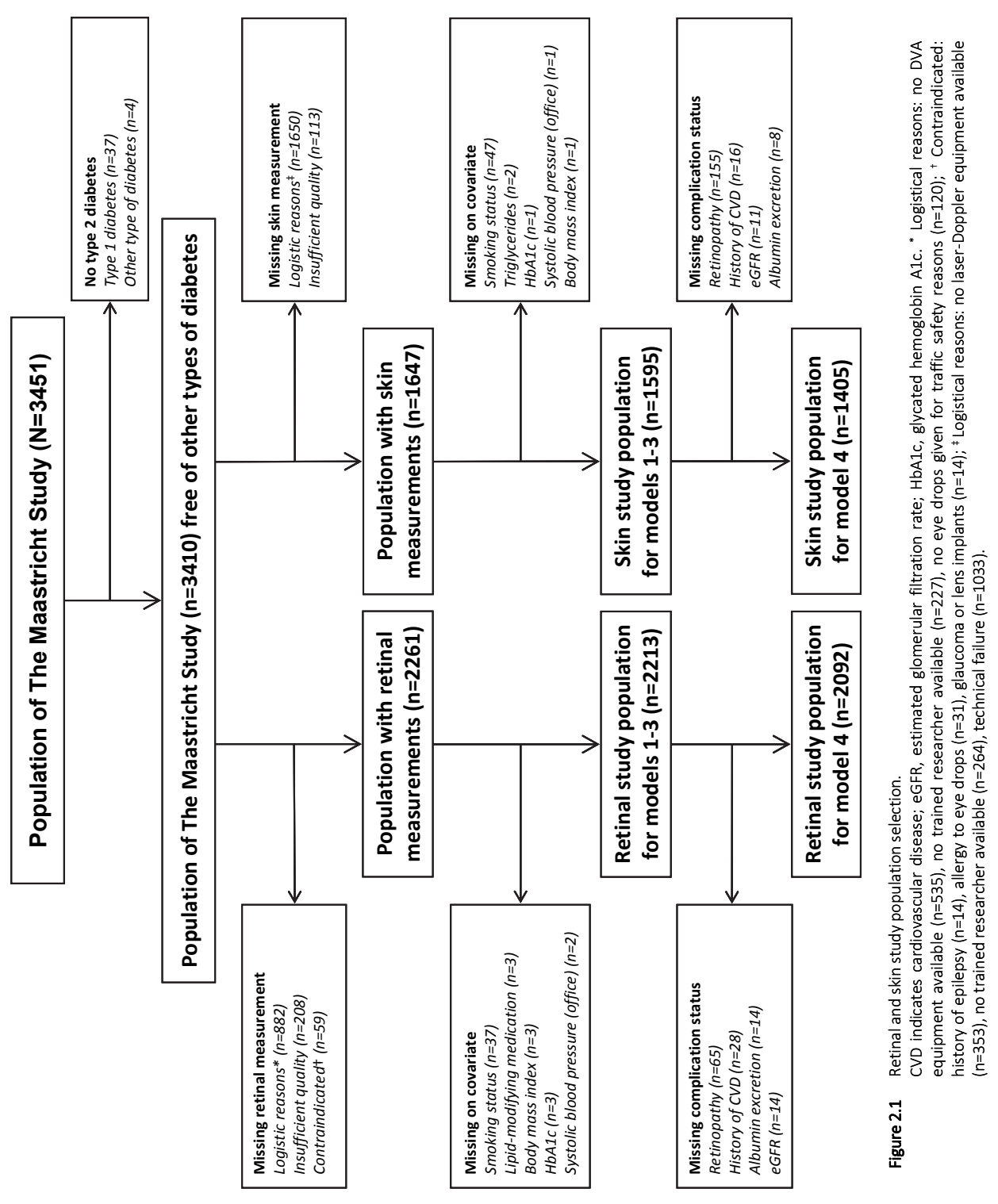




\section{Retinal arteriolar dilation response}

The retinal arteriolar dilation response to flicker light, which is thought to be related to nutritive demands of activated retinal neurons ${ }^{21}$, was measured in a dimly lit room by use of the Dynamic Vessel Analyzer (DVA) (Imedos, Jena, Germany). For safety reasons, participants with an intraocular pressure exceeding $30 \mathrm{mmHg}$ were excluded from retinal measurements. Per participant, we randomly measured the left or right eye.

During the measurement, the participant was instructed and encouraged to focus on the tip of a fixated needle inside the retinal camera (FF450; Carl Zeiss GmbH, Jena, Germany), while the fundus of the eye was examined under green measuring light (530-600 nm, illumination of fundus approximately 6500 lux). A straight arteriolar segment of approximately $1.5 \mathrm{~mm}$ in length located 0.5 to 2.0 disc diameter from the margin of the optic disc in the temporal section was examined. When the specific vessel profile was recognized, vessel diameter was automatically and continuously measured for 150 seconds. A baseline recording of 50 seconds was followed by a 40-second flicker light exposure period (flicker frequency $12.5 \mathrm{~Hz}$, bright-to-dark contrast ratio 25:1) followed by a 60-second recovery period. The DVA automatically corrected for alterations in luminance caused by, for example, slight eye movements. During blinks and small eye movements, the registration stopped and restarted once the vessel segment was automatically re-identified ${ }^{21}$.

The integrated DVA software (version 4.51, Imedos) automatically calculated baseline diameter and percentage dilation. Baseline diameter was calculated as the average diameter size of the 20-50 seconds recording and was expressed in measurement units (MU), where $1 \mathrm{MU}$ is equal to $1 \mu \mathrm{m}$ of the Gullstrand eye $\mathrm{e}^{22}$. Percentage dilation over baseline was based on the average dilation achieved at timepoints 10 and 40 seconds during the flicker stimulation period. Two regression lines were drawn (at interval 0-10 seconds and 10-40 seconds during flicker stimulation) and averaged to assess average percentage dilation (Figure 2.2A). The software successfully assessed two regression lines in $95.4 \%$ of the curves; only 102 dilation curves $(4.6 \%)$ were based on one regression line. The purpose of taking the average dilation was to account for inter-individual variation in the curve shape during dilation.

\section{Skin hyperemic response}

Skin blood flow was measured as described previously by means of a laser-Doppler system (Periflux 5000, Perimed, Järfälla, Sweden), equipped with a thermostatic laserDoppler probe (PF457; Perimed) at the dorsal side of the wrist of the left hand ${ }^{23}$. The laser-Doppler output was recorded for 25 minutes with a sample rate of $32 \mathrm{~Hz}$, which gives semi-quantitative assessment of skin blood flow expressed in arbitrary perfusion 


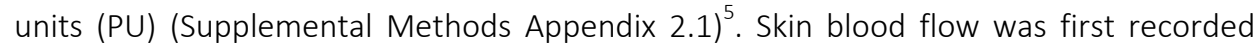
unheated for 2 minutes to serve as a baseline. After the 2 minutes of baseline, the temperature of the probe was rapidly and locally increased to $44^{\circ} \mathrm{C}$, and was then kept constant until the end of the registration. The heat-induced skin hyperemic response was expressed as the percentage increase in average PU during the 23 minutes heating phase over the average baseline PU (Figure 2.2B). The response is thought to be related to skin thermoregulatory function ${ }^{24}$.

A

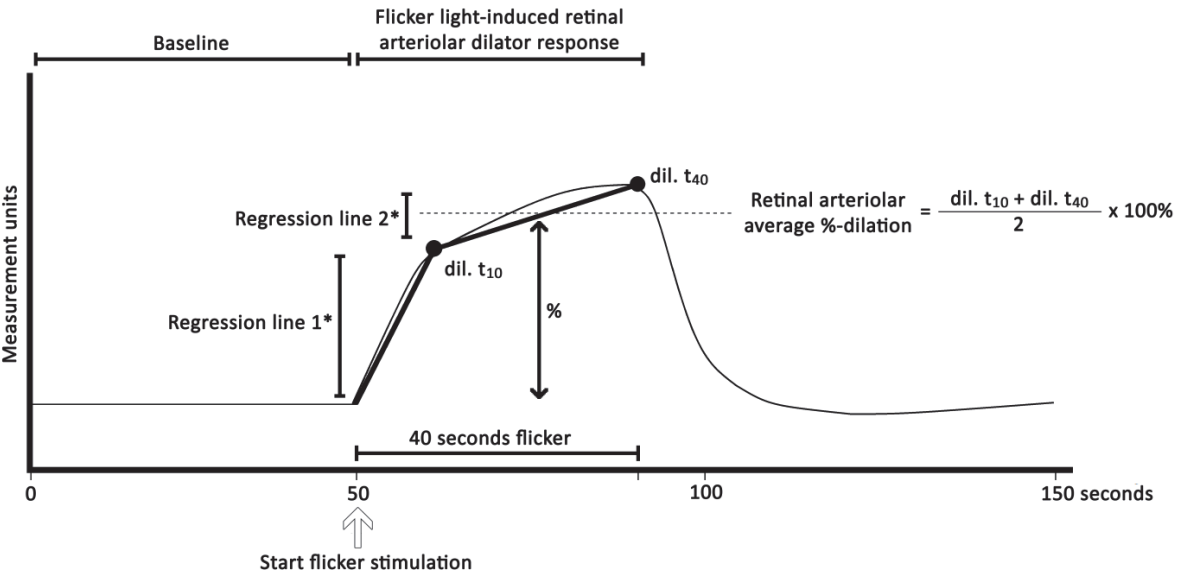

B

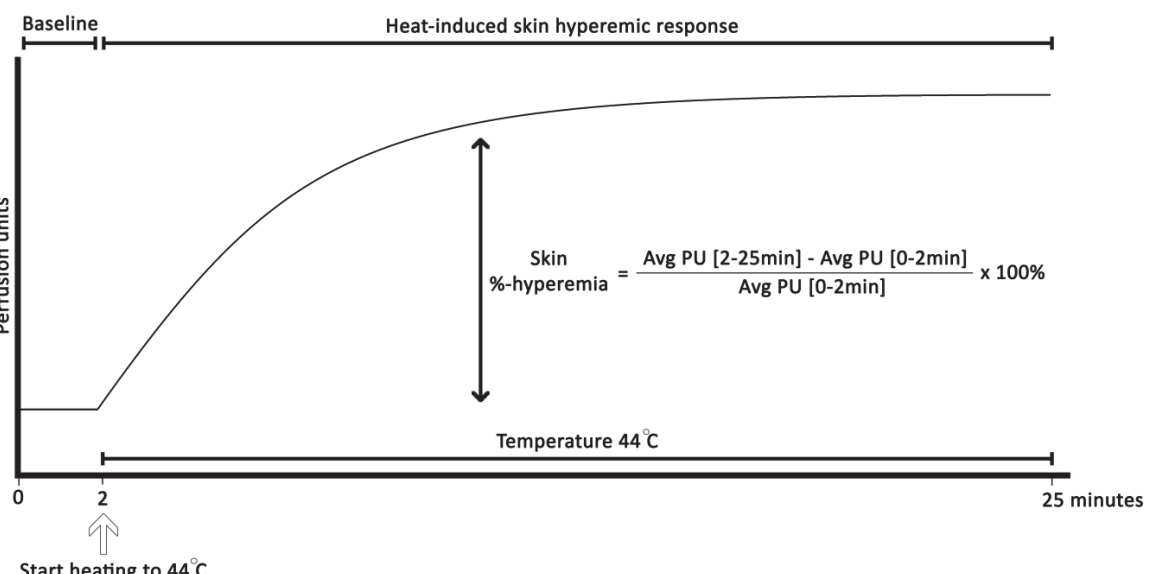

Figure 2.2 (A) Schematic Dynamic Vessel Analyzer registration of a flicker light-induced retinal arteriolar dilation response. (B) Schematic laser-Doppler registration of a heat-induced skin hyperemic response. Dil=dilation, Avg=average, $\mathrm{PU}=$ perfusion units, min=minutes. * Regression lines were drawn at interval 0-10 seconds and 10-40 seconds during flicker stimulation. 


\section{Validation of measurements}

Retinal and skin vasodilation measurements were performed by different observers, after an intensive training period. Inter-observer reliability (ICC) of the retinal arteriolar baseline diameter and percentage dilation response $(n=9)$ between two randomly selected observers were 0.980 and 0.796 , respectively. Retinal arteriolar dilation response curves were analyzed by one observer for measurement quality decisions, while heat-induced skin hyperemic response curves were analyzed by two observers ( $n=1760, I C C=0.839$ ). Retinal and skin response curves with insufficient measurement quality, e.g. insufficient measurement points or movement artifacts were evaluated and discussed with a second observer, and excluded on mutual agreement. To assess the ICC of retinal response curves quality decisions 50 curves were evaluated by two observers (ICC=0.883).

\section{Measurement of covariates}

History of cardiovascular disease, duration of diabetes, menopausal status, physical activity (hours/week), and smoking status (never, former, current) were assessed by questionnaire $^{18}$. Use of lipid-modifying, antihypertensive, and glucose-lowering medication as well as postmenopausal hormone replacement therapy was assessed during a medication interview where generic name, dose, and frequency were registered ${ }^{18}$. We measured weight, height, body mass index, waist circumference, office and ambulatory 24-h blood pressure, plasma glucose levels, serum creatinine, 24-h urinary albumin excretion (twice), glycated hemoglobin A1c (HbA1c), and plasma lipid profile as described elsewhere ${ }^{18}$. Estimated glomerular filtration rate (eGFR; in $\mathrm{ml} / \mathrm{min} / 1.73 \mathrm{~m}^{2}$ ) was calculated with the Chronic Kidney Disease Epidemiology Collaboration (CDK-epi) equation based on both serum creatinine and serum cystatin $\mathrm{C}^{25}$. The presence of retinopathy was based on fundus photographs taken with an auto fundus camera (Model AFC-230, Nidek, Gamagori, Japan) ${ }^{18}$.

\section{Statistical analysis}

All analyses were performed by use of the Statistical Package for Social Sciences version 22.0 (IBM SPSS, Armonk, USA). Multiple linear regression analysis was used to determine the association of glucose metabolism (NGM, prediabetes, and T2D), fasting plasma glucose (FPG), 2-h postload OGTT plasma glucose levels, or HbA1c with retinal arteriolar dilation and heat-induced skin hyperemia. For linear trend analyses, the categorical variable glucose metabolism status ( $\mathrm{NGM}=0$, prediabetes $=1$, and $\mathrm{T} 2 \mathrm{D}=2$ ) was used in the regression models. To assess regression coefficients per glucose metabolism group pairwise analyses with dummy variables for prediabetes and T2D were used. Model 1 
was the crude model, model 2 was adjusted for age and sex, and model 3 was additionally adjusted for cardiovascular risk factors that have previously been associated with altered vessel responses, and may therefore be potential confounders (body mass index, smoking status, systolic blood pressure, physical activity, use of antihypertensive and/or lipid-modifying drugs, fasting triglycerides, and total-to-HDL-cholesterol levels). In model 4 we additionally adjusted for history of cardiovascular disease, retinopathy, eGFR, and urinary albumin excretion (the latter two as continuous variables). A P-value $<0.05$ was considered statistically significant. Interaction terms (e.g. prediabetes $*$ sex or $\mathrm{HbA} 1 \mathrm{c} * \mathrm{sex}$ ) were incorporated in the regression models to test for interaction between, on the one hand, prediabetes, T2D, and measures of hyperglycemia, and, on the other hand, sex, on retinal arteriolar dilation and heat-induced skin hyperemia. A $\mathrm{P}_{\text {interaction }}<0.10$ was considered statistically significant.

\section{Results}

\section{Characteristics of study population}

General characteristics of the population in which retinal reactivity data were available are shown in Table 2.1, stratified for glucose metabolism status. The study population consisted of 2213 individuals with a mean \pm standard deviation (SD) age of $59.7 \pm 8.2$ years of whom $51.1 \%$ were men. Individuals with T2D were, by design, oversampled (27.5\%). The cardiometabolic risk profile deteriorated with glucose metabolism status. Individuals with T2D were older, more often male and/or current smoker and had a higher body mass index, waist circumference, blood pressure, FPG and triglycerides levels, and less physical activity, than individuals without T2D (Table 2.1). The population in which heat-induced skin hyperemia data were available ( $n=1595)$, overlapped for $73 \%$ with the population in which retinal reactivity data were available, and was comparable with regard to age, sex, and cardiometabolic risk profile (Supplemental Tables S2.1 and S2.2). Individuals with missing data on retinal or skin reactivity measurements or covariates were to a great extent comparable with regard to age, sex, and cardiometabolic risk profile as compared to individuals included in the study populations (Supplemental Tables S2.1 and S2.2). 
Table 2.1 General characteristics and retinal and skin measures of the retinal study population according to glucose metabolism status

\begin{tabular}{|c|c|c|c|}
\hline Characteristic & $\begin{array}{c}\mathrm{NGM} \\
\mathrm{n}=1269\end{array}$ & $\begin{array}{l}\text { Prediabetes } \\
\quad \mathrm{n}=335\end{array}$ & $\begin{array}{c}\text { Type } 2 \text { diabetes } \\
\mathrm{n}=609\end{array}$ \\
\hline Age (years) & $57.8 \pm 8.2$ & $61.3 \pm 7.5$ & $62.8 \pm 7.6$ \\
\hline Women & $737(58.1)$ & $155(46.3)$ & $191(31.4)$ \\
\hline - Postmenopausal & $542(75.2)$ & $121(81.2)$ & $148(87.6)$ \\
\hline - Hormone replacement therapy & $13(1.8)$ & $2(1.3)$ & $3(1.6)$ \\
\hline Diabetes duration (years) ${ }^{*}$ & - & - & $8.2 \pm 7.0$ \\
\hline Body mass index $\left(\mathrm{kg} / \mathrm{m}^{2}\right)$ & $25.4 \pm 3.5$ & $27.5 \pm 4.3$ & $29.8 \pm 4.9$ \\
\hline \multicolumn{4}{|l|}{ Waist circumference $(\mathrm{cm})$} \\
\hline- Men & $96.1 \pm 9.2$ & $101.9 \pm 10.8$ & $107.4 \pm 12.7$ \\
\hline - Women & $85.5 \pm 9.9$ & $92.2 \pm 12.4$ & $101.3 \pm 13.9$ \\
\hline History of cardiovascular disease & $141(11.3)$ & $40(12.0)$ & $158(26.3)$ \\
\hline Office SBP (mmHg) & $130.9 \pm 17.3$ & $137.3 \pm 17.2$ & $142.1 \pm 17.6$ \\
\hline Office DBP (mmHg) & $75.4 \pm 9.9$ & $78.1 \pm 9.7$ & $77.4 \pm 9.6$ \\
\hline Ambulatory 24-h SBP (mmHg) & $117.3 \pm 11.4$ & $119.9 \pm 11.2$ & $122.5 \pm 11.7$ \\
\hline Ambulatory 24-h DBP (mmHg) & $73.6 \pm 7.2$ & $74.4 \pm 7.2$ & $72.9 \pm 7.0$ \\
\hline Physical activity (hours/week) & $14.8 \pm 8.0$ & $14.6 \pm 8.0$ & $11.9 \pm 7.5$ \\
\hline \multicolumn{4}{|l|}{ Smoking } \\
\hline - Never / former / current & $508 / 614 / 147$ & 95/199/41 & $177 / 346 / 86$ \\
\hline - \% (never / former / current) & $40.0 / 48.4 / 11.6$ & $28.4 / 59.4 / 12.2$ & $29.1 / 56.8 / 14.1$ \\
\hline Fasting glucose $(\mathrm{mmol} / \mathrm{l})$ & $5.2 \pm 0.4$ & $5.9 \pm 0.6$ & $7.9 \pm 2.1$ \\
\hline 2-h postload glucose $(\mathrm{mmol} / \mathrm{l})^{+}$ & $5.4 \pm 1.1$ & $8.1 \pm 1.7$ & $14.3 \pm 3.9$ \\
\hline HbA1c (\%) & $5.4 \pm 0.3$ & $5.7 \pm 0.4$ & $6.9 \pm 1.0$ \\
\hline $\mathrm{HbA} 1 \mathrm{c}(\mathrm{mmol} / \mathrm{mol})$ & $35.7 \pm 3.7$ & $38.5 \pm 4.5$ & $51.7 \pm 11.4$ \\
\hline Triglycerides (mmol/l) & $1.2 \pm 0.6$ & $1.6 \pm 1.0$ & $1.8 \pm 1.0$ \\
\hline Total-to-HDL cholesterol ratio & $3.5 \pm 1.1$ & $3.8 \pm 1.2$ & $3.7 \pm 1.1$ \\
\hline Total cholesterol (mmol/l) & $5.6 \pm 1.0$ & $5.5 \pm 1.2$ & $4.4 \pm 1.0$ \\
\hline HDL cholesterol (mmol/l) & $1.7 \pm 0.5$ & $1.5 \pm 0.4$ & $1.3 \pm 0.4$ \\
\hline LDL cholesterol (mmol/l) & $3.3 \pm 0.9$ & $3.2 \pm 1.0$ & $2.4 \pm 0.9$ \\
\hline Antihypertensive medication use & $266(21.0)$ & $141(42.1)$ & 438 (71.9) \\
\hline Lipid-modifying medication use & $203(16.0)$ & $117(34.9)$ & $454(74.5)$ \\
\hline Diabetes medication use & $0(0)$ & $0(0)$ & $476(78.2)$ \\
\hline - Insulin & - & - & $121(19.9)$ \\
\hline - Metformin & - & - & $423(69.5)$ \\
\hline - Sulfonylureas & - & - & $119(19.5)$ \\
\hline - Thiazolidinediones & - & - & $6(1.0)$ \\
\hline - GLP-1 analogs & - & - & $3(0.5)$ \\
\hline - DPP-4 inhibitors & - & - & $43(7.1)$ \\
\hline $\operatorname{eGFR}\left(\mathrm{ml} / \mathrm{min} / 1.73 \mathrm{~m}^{2}\right)$ & $90.3 \pm 13.0$ & $87.2 \pm 13.7$ & $84.7 \pm 17.3$ \\
\hline eGFR<60 $\left(\mathrm{ml} / \mathrm{min} / 1.73 \mathrm{~m}^{2}\right)$ & $19(1.5)$ & $11(3.3)$ & $62(10.3)$ \\
\hline (Micro)albuminuria ${ }^{\ddagger}$ & $55(4.4)$ & $18(5.4)$ & $106(17.4)$ \\
\hline Retinopathy & $1(0.1)$ & $1(0.3)$ & $25(4.2)$ \\
\hline Baseline arteriolar diameter (MU) & $115.5 \pm 15.5$ & $114.5 \pm 15.8$ & $115.9 \pm 15.9$ \\
\hline \multicolumn{4}{|l|}{ Arteriolar average dilation (\%) } \\
\hline - Mean \pm SD & $3.4 \pm 2.8$ & $3.0 \pm 2.7$ & $2.3 \pm 2.6$ \\
\hline - Median [interquartile range] & $3.1[1.2-5.3]$ & $2.7[0.8-4.9]$ & $1.5[0.4-3.8]$ \\
\hline Baseline skin blood flow $(P U)^{\S}$ & $11.1 \pm 6.8$ & $11.5 \pm 6.8$ & $10.9 \pm 5.6$ \\
\hline \multicolumn{4}{|l|}{ Skin hyperemic response $(\%)^{\S}$} \\
\hline- Mean \pm SD & $1234.9 \pm 810.4$ & $1108.7 \pm 747.9$ & $936.7 \pm 683.2$ \\
\hline - Median [interquartile range] & $1097.4[646.8-1630.7]$ & $995.5[567.0-1518.5]$ & $821.3[480.0-1207.8]$ \\
\hline
\end{tabular}

Data are reported as mean \pm SD or number (percentage \%) as appropriate. Data present the retinal study population for regression models 1-3. NGM, normal glucose metabolism; SBP, systolic blood pressure; DBP, diastolic blood pressure; HbA1c, glycated hemoglobin A1c; HDL, high-density lipoprotein; LDL, low-density lipoprotein; GLP, glucagon-like peptide; DPP-4, dipeptidyl peptidase-4; eGFR, estimated glomerular filtration rate; MU, measurement units; PU, perfusion units. "Available in $420 \mathrm{~T} 2 \mathrm{D}$ individuals. ${ }^{+}$Available in $471 \mathrm{~T} 2 \mathrm{D}$ individuals. ${ }^{\ddagger}$ (Micro)albuminuria was defined as a urinary albumin excretion of $>30 \mathrm{mg}$ per 24 hours. ${ }^{\S}$ heat-induced skin hyperemia measures were available in a different subset of $n=1595$ (Supplemental Table S2.1). 


\section{Glucose metabolism status and flicker light-induced retinal arteriolar dilation}

Arteriolar baseline diameter did not significantly differ among the three groups of glucose metabolism in crude or adjusted analyses, while the average \%-dilation was lower in individuals with prediabetes and T2D as compared to NGM ( $P$ for trend $<0.001$, Figure 2.3A and Table 2.2). Adjustment for age and sex attenuated the difference in \%-dilation between individuals with T2D or prediabetes and NGM, but the association remained statistically significant $(P$ for trend $<0.001$ ). Additional adjustment for cardiovascular risk factors (model 3) and for history of cardiovascular disease, retinopathy, eGFR, and urinary albumin excretion (model 4) further attenuated the difference in \%-dilation between individuals with T2D or prediabetes and NGM, but the association again remained statistically significant, with the unstandardized regression coefficient of prediabetes consistently $\sim 1 / 2$ to $1 / 4$ of the T2D coefficient (Figure 2.3A and Table 2.2). Possible confounders, in the fully adjusted model, that were associated with the retinal dilator response were age $(B=-0.04,95 \%$ confidence interval $(95 \% \mathrm{Cl})$ $(-0.06 ;-0.02))$, systolic blood pressure $(B=0.01,(0.00 ; 0.02))$, triglycerides $(B=-0.18$, $(-0.37 ; 0.01))$, and retinopathy $(B=-1.34,(-2.41 ;-0.26))$; betas indicate the change in $\%$-dilation per 1 unit higher age, systolic blood pressure, and triglycerides, or when having retinopathy.

\section{Glucose metabolism status and heat-induced skin hyperemic outcomes}

Baseline skin blood flow did not significantly differ among the three groups of glucose metabolism in crude or adjusted analyses, while the hyperemic response was lower in individuals with prediabetes and T2D as compared to NGM ( $P$ for trend $<0.001$, Figure 2.3B and Table 2.2). Adjustment for age and sex attenuated the difference in hyperemic response between individuals with T2D or prediabetes and NGM, but the association remained statistically significant ( $P$ for trend $<0.001$ ). Additional adjustment for cardiovascular risk factors (model 3) and for history of cardiovascular disease, retinopathy, eGFR, and urinary albumin excretion (model 4) further attenuated the difference in hyperemic response between individuals with T2D or prediabetes and NGM, but the association again remained statistically significant, with the unstandardized regression coefficient of prediabetes consistently $\sim 1 / 2$ to $1 / 4$ of the T2D coefficient (Figure 2.3B and Table 2.2). Possible confounders, in the fully adjusted model, that were significantly associated with the skin hyperemic response were age $(B=-12$, $95 \% \mathrm{Cl}(-19 ;-6))$, female sex $(B=311,(224 ; 397))$, body mass index $(B=11,(1 ; 21))$, smoking status $(B=-265,(-398 ;-132))$, systolic blood pressure $(B=3,(0 ; 5))$, and triglycerides $(B=-89,(-144 ;-33))$; betas indicate the change in \%-hyperemia per unit higher age, body mass index, systolic blood pressure, and triglycerides, or being female or a current (versus never) smoker. 
A

Flicker light-induced retinal arteriolar \%-dilation

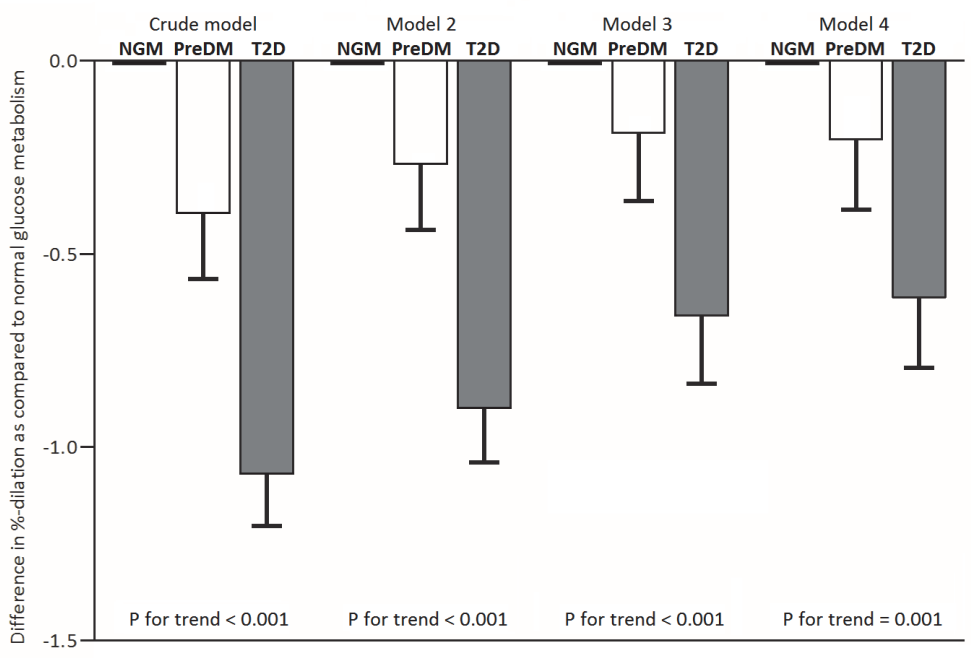

B

Heat-induced skin \%-hyperemia

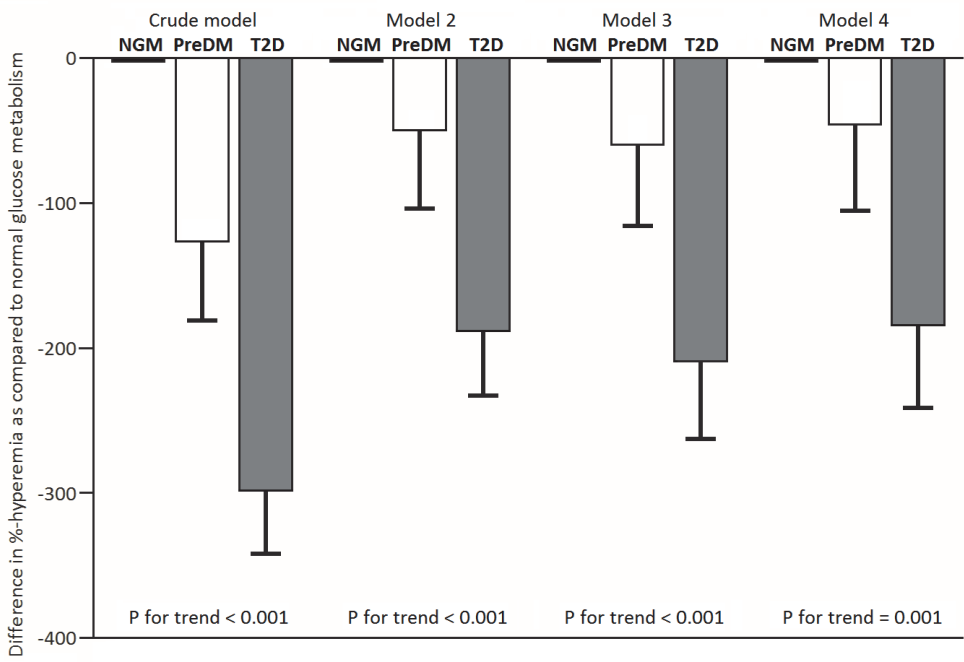

Figure 2.3 Multivariable-adjusted differences in flicker light-induced retinal arteriolar \%-dilation (A) and heat-induced skin \%-hyperemia (B) between individuals with prediabetes (PreDM) and type 2 diabetes (T2D) compared to normal glucose metabolism (NGM).

Bars represent the mean difference with standard error in retinal arteriolar \%-dilation or heatinduced skin \%-hyperemia for prediabetes and T2D as compared to NGM. P-values indicate trend analyses among NGM, prediabetes, and T2D. NGM is the reference and is set to zero. Model 2: adjusted for age and sex. Model 3: additionally adjusted for body mass index, triglyceride levels, total-to-HDL-cholesterol ratio, smoking status, systolic blood pressure, and use of antihypertensive and/or lipid-modifying drugs. Model 4: additionally adjusted for history of cardiovascular disease, retinopathy, eGFR, and urinary albumin excretion. 
Table 2.2 Multivariable-adjusted differences in retinal arteriolar baseline diameter, flicker light-induced retinal arteriolar \%-dilation, skin baseline blood flow, and heat-induced skin \%-hyperemia among individuals with normal glucose metabolism (NGM), prediabetes, and type 2 diabetes

\begin{tabular}{|c|c|c|c|}
\hline Characteristic & Prediabetes* & Type 2 diabetes* & P for trend \\
\hline Retinal arteriolar baseline diameter (MU) & $\mathrm{B}(95 \% \mathrm{Cl})$ & $\mathrm{B}(95 \% \mathrm{Cl})$ & \\
\hline Crude & $-1.02(-2.91 ; 0.86)$ & $0.39(-1.12 ; 1.90)$ & 0.749 \\
\hline Model 2 & $-0.69(-2.60 ; 1.22)$ & $0.91(-0.69 ; 2.51)$ & 0.334 \\
\hline Model 3 & $-0.62(-2.58 ; 1.35)$ & $0.84(-1.13 ; 2.80)$ & 0.490 \\
\hline Model 4 & $-0.05(-2.06 ; 1.96)$ & $0.83(-1.20 ; 2.86)$ & 0.457 \\
\hline Retinal arteriolar average dilation (\%) & $\mathrm{B}(95 \% \mathrm{Cl})$ & $\mathrm{B}(95 \% \mathrm{Cl})$ & \\
\hline Crude & $-0.39(-0.73 ;-0.06)$ & $-1.07(-1.34 ;-0.80)$ & $<0.001$ \\
\hline Model 2 & $-0.27(-0.60 ; 0.07)$ & $-0.90(-1.18 ;-0.62)$ & $<0.001$ \\
\hline Model 3 & $-0.19(-0.53 ; 0.16)$ & $-0.66(-1.01 ;-0.31)$ & $<0.001$ \\
\hline Model 4 & $-0.20(-0.56 ; 0.15)$ & $-0.61(-0.97 ;-0.25)$ & 0.001 \\
\hline Retinal arteriolar average dilation (SD) & stB $(95 \% \mathrm{Cl})$ & stB $(95 \% \mathrm{Cl})$ & \\
\hline Crude & $-0.05(-0.09 ;-0.01)$ & $-0.17(-0.21 ;-0.13)$ & $<0.001$ \\
\hline Model 2 & $-0.03(-0.08 ; 0.01)$ & $-0.14(-0.19 ;-0.10)$ & $<0.001$ \\
\hline Model 3 & $-0.02(-0.07 ; 0.02)$ & $-0.11(-0.16 ;-0.05)$ & $<0.001$ \\
\hline Model 4 & $-0.03(-0.07 ; 0.02)$ & $-0.10(-0.16 ;-0.04)$ & 0.001 \\
\hline Skin baseline blood flow (PU) & $\mathrm{B}(95 \% \mathrm{Cl})$ & $\mathrm{B}(95 \% \mathrm{Cl})$ & \\
\hline Crude & $0.37(-0.53 ; 1.27)$ & $-0.19(-0.91 ; 0.53)$ & 0.689 \\
\hline Model 2 & $0.05(-0.87 ; 0.96)$ & $-0.58(-1.33 ; 0.18)$ & 0.151 \\
\hline Model 3 & $0.35(-0.59 ; 1.28)$ & $0.00(-0.90 ; 0.91)$ & 0.930 \\
\hline Model 4 & $0.15(-0.86 ; 1.17)$ & $-0.25(-1.23 ; 0.72)$ & 0.655 \\
\hline Heat-induced skin hyperemia (\%) & $\mathrm{B}(95 \% \mathrm{Cl})$ & $\mathrm{B}(95 \% \mathrm{Cl})$ & \\
\hline Crude & $-126(-233 ;-19)$ & $-298(-384 ;-213)$ & $<0.001$ \\
\hline Model 2 & $-49(-156 ; 57)$ & $-188(-276 ;-100)$ & $<0.001$ \\
\hline Model 3 & $-60(-169 ; 50)$ & $-209(-314 ;-104)$ & $<0.001$ \\
\hline Model 4 & $-46(-163 ; 72)$ & $-184(-297 ;-71)$ & 0.001 \\
\hline Heat-induced skin hyperemia (SD) & stB $(95 \% \mathrm{Cl})$ & stB $(95 \% \mathrm{Cl})$ & \\
\hline Crude & $-0.06(-0.11 ;-0.01)$ & $-0.18(-0.23 ;-0.13)$ & $<0.001$ \\
\hline Model 2 & $-0.02(-0.07 ; 0.03)$ & $-0.11(-0.16 ;-0.06)$ & $<0.001$ \\
\hline Model 3 & $-0.03(-0.08 ; 0.02)$ & $-0.12(-0.19 ;-0.06)$ & $<0.001$ \\
\hline Model 4 & $-0.02(-0.08 ; 0.03)$ & $-0.11(-0.18 ;-0.04)$ & 0.001 \\
\hline
\end{tabular}

Regression coefficients $(B)$ indicate the mean difference $(95 \% \mathrm{Cl}$ ) in retinal and skin measures (in \%) with NGM as reference. Standardized regression coefficients (stB) and $95 \% \mathrm{Cl}$ represent the change in retinal and skin measures (in SD) as compared to NGM. SD, standard deviation; $\mathrm{Cl}$, confidence interval.

Model 2: adjusted for age and sex. Model 3: additionally adjusted for body mass index, triglyceride levels, total-to-HDL-cholesterol ratio, smoking status, systolic blood pressure, and use of antihypertensive and/or lipid-modifying drugs. Model 4: additionally adjusted for history of cardiovascular disease, retinopathy, eGFR, and urinary albumin excretion. ${ }^{*}$ The retinal reactivity population consisted of 335 individuals with prediabetes and 609 with type 2 diabetes, the heat-induced skin hyperemia population consisted of 254 individuals with prediabetes and 478 with type 2 diabetes.

\section{Associations of measures of hyperglycemia with retinal arteriolar dilation and heat-induced skin hyperemia}

$\mathrm{HbA} 1 \mathrm{c}, \mathrm{FPG}$, and 2-h postload glucose levels were not associated with baseline retinal arteriolar diameter or baseline skin blood flow, either in crude or adjusted models (data not shown). In contrast, both $\mathrm{HbA1c}$ and FPG were associated with the average 
percentage dilation and hyperemic response, both in crude and adjusted models (Table 2.3 and Figure 2.4). The association of 2-h postload glucose levels with retinal arteriolar percentage dilation was non-significant after adjustment for cardiovascular risk factors and remained significant for the skin hyperemic response (Table 2.3 and Figure 2.4).

Table 2.3 Multivariable-adjusted associations of $\mathrm{HbA} 1 \mathrm{c}$, fasting glucose, and 2-h postload glucose levels with the flicker light-induced retinal arteriolar dilator and the heat-induced skin hyperemic response

\begin{tabular}{|c|c|c|c|c|c|c|}
\hline \multirow[b]{2}{*}{ Characteristic } & \multicolumn{2}{|l|}{$\mathrm{HbA1c}$} & \multicolumn{2}{|c|}{ Fasting glucose } & \multicolumn{2}{|c|}{ 2-h postload glucose } \\
\hline & stB $(95 \% \mathrm{Cl})$ & P-value & stB $(95 \% \mathrm{Cl})$ & P-value & stB $(95 \% \mathrm{Cl})$ & P-value \\
\hline \multirow{2}{*}{\multicolumn{7}{|c|}{$\begin{array}{l}\text { Retinal arteriolar } \\
\text { average dilation (\%) }\end{array}$}} \\
\hline & & & & & & \\
\hline Crude & $-0.17(-0.21 ;-0.13)$ & $<0.001$ & $-0.16(-0.20 ;-0.12)$ & $<0.001$ & $-0.11(-0.15 ;-0.07)$ & $<0.001$ \\
\hline Model 2 & $-0.15(-0.19 ;-0.11)$ & $<0.001$ & $-0.14(-0.18 ;-0.10)$ & $<0.001$ & $-0.08(-0.13 ;-0.04)$ & $<0.001$ \\
\hline Model 3 & $-0.12(-0.17 ;-0.07)$ & $<0.001$ & $-0.11(-0.16 ;-0.06)$ & $<0.001$ & $-0.05(-0.10 ; 0.00)$ & 0.054 \\
\hline Model 4 & $-0.10(-0.15 ;-0.05)$ & $<0.001$ & $-0.09(-0.15 ;-0.04)$ & $<0.001$ & $-0.04(-0.10 ; 0.01)$ & 0.123 \\
\hline \multirow{2}{*}{\multicolumn{7}{|c|}{$\begin{array}{l}\text { Heat-induced skin } \\
\text { hyperemia (\%) }\end{array}$}} \\
\hline & & & & & & \\
\hline Crude & $-0.17(-0.21 ;-0.12)$ & $<0.001$ & $-0.16(-0.20 ;-0.11)$ & $<0.001$ & $-0.14(-0.19 ;-0.09)$ & $<0.001$ \\
\hline Model 2 & $-0.12(-0.17 ;-0.07)$ & $<0.001$ & $-0.10(-0.15 ;-0.05)$ & $<0.001$ & $-0.08(-0.13 ;-0.03)$ & 0.002 \\
\hline Model 3 & $-0.13(-0.18 ;-0.07)$ & $<0.001$ & $-0.10(-0.15 ;-0.04)$ & 0.001 & $-0.10(-0.16 ;-0.04)$ & 0.001 \\
\hline Model 4 & $-0.13(-0.19 ;-0.07)$ & $<0.001$ & $-0.10(-0.15 ;-0.04)$ & 0.002 & $-0.09(-0.15 ;-0.02)$ & 0.007 \\
\hline
\end{tabular}

Point estimates (standardized betas) and $95 \% \mathrm{Cls}$ represent the change in flicker light-induced retinal arteriolar \%-dilation (in SD) and heat-induced skin \%-hyperemia (in SD) per SD increase in HbA1c, fasting plasma glucose, or 2-h postload glucose level. stB; standardized beta, $\mathrm{Cl}$, confidence interval; SD, standard deviation.

Model 2: adjusted for age and sex. Model 3: additionally adjusted for body mass index, triglyceride levels, total-to-HDL-cholesterol ratio, smoking status, systolic blood pressure, and use of antihypertensive and/or lipid-modifying drugs. Model 4: additionally adjusted for history of cardiovascular disease, retinopathy, eGFR, and urinary albumin excretion. 


\section{A}

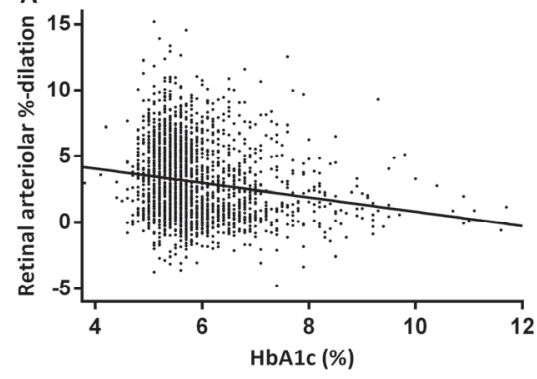

$\mathrm{B}(95 \% \mathrm{Cl})=-0.32(-0.48 ;-0.16), \mathrm{P}<0.001$

C

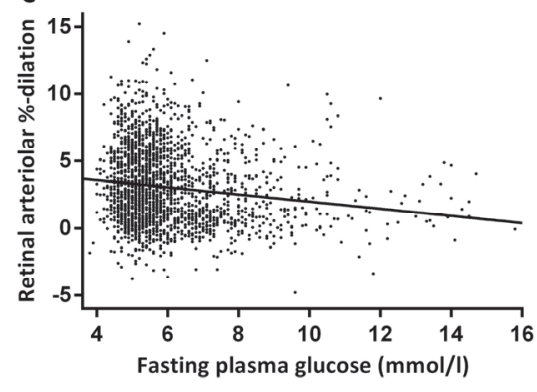

B $(95 \% \mathrm{Cl})=-0.16(-0.24 ;-0.07), \mathrm{P}<0.001$

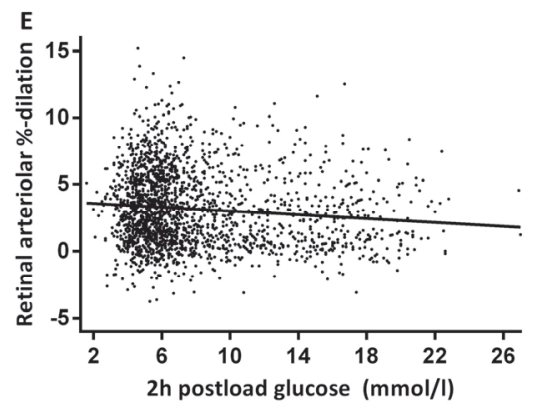

$B(95 \% C l)=-0.03(-0.06 ; 0.01), P=0.123$
B

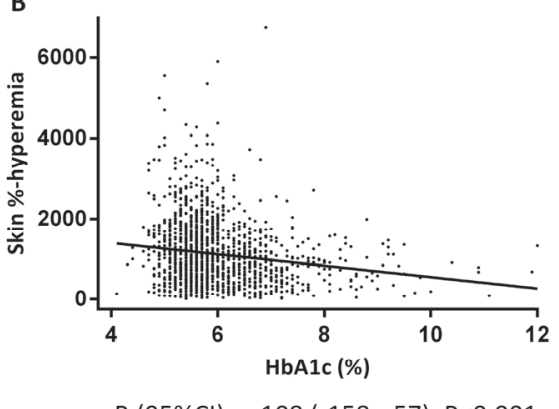

D

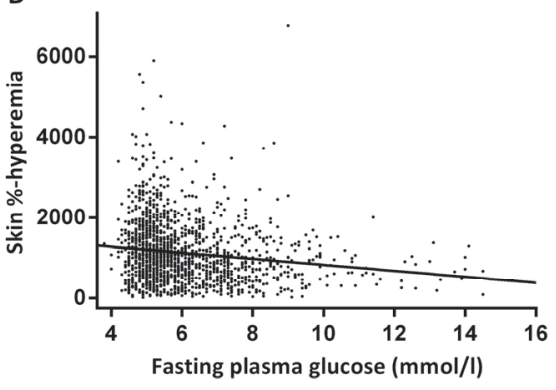

$B(95 \% C l)=-45(-74 ;-17), P=0.002$

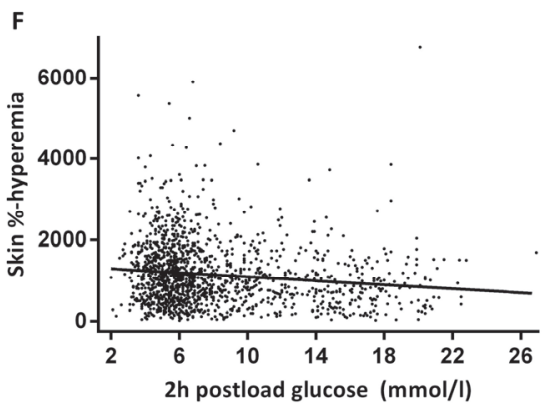

$B(95 \% C l)=-16(-27 ;-4), \quad P=0.007$

Figure 2.4 Associations of $\mathrm{HbA1c}$ (top), fasting plasma glucose (middle), and 2-h postload glucose (bottom) with the flicker light-induced retinal arteriolar dilator (left) and heat-induced skin hyperemic response (right).

Association between $\mathrm{HbA1C}$ and flicker light-induced retinal arteriolar \%-dilation (A), Association between $\mathrm{HbA1c}$ and heat-induced skin \%-hyperemia (B), Association between fasting plasma glucose and flicker light-induced retinal arteriolar \%-dilation (C), Association between fasting plasma glucose and heat-induced skin \%-hyperemia (D), Association between 2-h postload glucose and flicker light-induced retinal arteriolar \%-dilation (E), Association between 2-h postload glucose and heat-induced skin \%-hyperemia (F). Regression coefficients (B) indicate the adjusted mean difference and $95 \%$ confidence interval $(95 \% \mathrm{Cl})$ in retinal arteriolar \%-dilation and skin \%-hyperemia per one percent point increase in $\mathrm{HbA} 1 \mathrm{c}$ or per $\mathrm{mmol} / \mathrm{l}$ increase in fasting glucose or 2-h postload glucose level. Note that the regression coefficients between retinal and skin responses differ due to differences in the scales of the response effect size. HbA1c indicates glycated hemoglobin A1c. 


\section{Additional analyses}

With regard to the retinal arteriolar dilation analyses, the associations of prediabetes and T2D, fasting glucose, 2-h postload glucose, or HbA1c with average percentage dilation remained unchanged after excluding participants of whom the average percentage dilation was based on 1 instead of 2 regression lines ( $n=102$, data not shown). Second, no significant differences were found between average percentage dilation measured in the right versus the left eye $(3.0 \pm 2.7 \%$ and $3.1 \pm 2.9 \%$, respectively, $\mathrm{P}=0.153)$. Third, qualitatively similar associations of prediabetes, $\mathrm{T} 2 \mathrm{D}$, fasting glucose, 2-h postload glucose, or $\mathrm{HbA} 1 \mathrm{c}$ with the retinal arteriolar dilator response were found when using absolute retinal arteriolar diameter corrected for baseline arteriolar diameter (data not shown). Similar associations also remained when substituting office systolic blood pressure for 24-h ambulatory systolic blood pressure (for retinal analyses, 24-h ambulatory blood pressure was available in $n=1962$ individuals; Supplemental Table S2.3), or adding physical activity (for retinal analyses, physical activity data were available in $n=1974$ individuals; Supplemental Table S2.4), or further specification of blood pressure-lowering medication into renin-angiotensin-aldosterone system (RAAS) inhibitors and other types of antihypertensives in the regression models (Supplemental Table 2.5). Fourth, analyses excluding T2D participants with microvascular complications (retinopathy, eGFR<60 ml/min $/ 1.73 \mathrm{~m}^{2}$, and/or urinary albumin excretion $>30$ mg/24 hour; Supplemental Table S2.6), or using 'newer' types of anti-diabetic medication (such as glucagon-like peptide-1 (GLP-1) analogs, thiazolidinediones, or dipeptidyl peptidase-4 (DPP-4) inhibitors; Supplemental Table S2.7), gave similar results with regard to the associations of prediabetes and T2D, fasting glucose, 2-h postload glucose, or HbA1c with average percentage dilation.

Fifth, with regard to heat-induced skin hyperemia analyses, qualitatively similar associations of prediabetes, T2D, fasting glucose, 2-h postload glucose, or HbA1c with the heat-induced skin hyperemic response were found when using absolute skin blood flow corrected for baseline skin blood flow (data not shown). Similar associations also remained when substituting office systolic blood pressure for 24-h ambulatory systolic blood pressure (for skin analyses, 24-h ambulatory blood pressure was available in $\mathrm{n}=1402$ individuals; Supplemental Table S2.3), or adding physical activity (for skin analyses, physical activity data were available in $n=1406$ individuals; Supplemental Table S2.4), or further specification of blood pressure-lowering medication into RAAS inhibitors and other types of antihypertensives in the regression models (Supplemental Table S2.5). Sixth, analyses excluding T2D participants with microvascular complications (Supplemental Table S2.6), or using 'newer' types of anti-diabetic medication (Supplemental Table S2.7), gave similar results with regard to the associations of 
prediabetes and T2D, fasting glucose, 2-h postload glucose, or HbA1c with the heatinduced skin hyperemic response.

Seventh, qualitatively similar associations of prediabetes, T2D, fasting glucose, 2-h postload glucose, or HbA1c with the flicker light-induced retinal arteriolar dilation and heat-induced skin hyperemic response were found when excluding outliers (defined as $<3 S D$ or $>3 S D ; n=11$ for retinal measures, $n=25$ for skin measures) (data not shown) or when selecting individuals who both had a retinal and skin measurement $(n=1162)$ (data not shown). Finally, the associations between prediabetes, T2D, and measures of hyperglycemia with flicker light-induced retinal arteriolar dilation and heat-induced skin hyperemia did not statistically significantly differ between men and women (P-values for interaction $>0.140$ ). Moreover, additional adjustment for postmenopausal status and/or hormone replacement therapy in women did not influence the associations of prediabetes, T2D, fasting glucose, 2-h postload glucose, or HbA1c with flicker lightinduced retinal arteriolar dilation or heat-induced skin hyperemia (Supplemental Table S2.8).

\section{Discussion}

This study demonstrates that both prediabetes and T2D are associated with microvascular dysfunction in retina and skin. In addition, measures of glycemia (HbA1c, fasting or 2-h postload glucose levels) are linearly associated with microvascular dysfunction. These associations were independent of major cardiovascular risk factors and clinically defined diabetic retinopathy and nephropathy. Taken together, these data support the concept that generalized microvascular dysfunction occurs prior to the diagnosis of T2D and may play a role in disorders that are (in part) of microvascular origin and that may occur early in the course of T2D and indeed in prediabetes, notably retinopathy, nephropathy, neuropathy, heart failure, stroke, and cognitive decline.

This is the first large, population-based study to show that microvascular dysfunction is a feature of prediabetes and T2D. Importantly, and in contrast to previous, smaller studies $^{12,13,26-28}$, we showed that these findings were independent of a broad array of potential confounders, which, as compared to age- and sex-adjusted estimates, explained about 2 to $32 \%$ of the associations between (pre)diabetes and microvascular dysfunction (Table 2.2 and Supplemental Table S2.4-S2.6). In addition, we showed that baseline values of retinal arteriolar diameters and skin perfusion were similar among the groups, and thus did not explain group differences in microvascular responses. Retinal arteriolar diameters were obtained in a single, temporally located vessel; our data therefore do not necessarily contradict prior findings of wider central retinal arteriolar 
equivalent (CRAE) in prevalent diabetes ${ }^{16}$, as CRAE represents averaged diameter of at least six arterioles radiating from the optic disc, whether temporally or nasally located.

We used linear trend analyses to test for a graded decline in microvascular function from NGM to prediabetes to T2D, as a similar trend has been shown for macrovascular function $^{6-8}$. Indeed, the decline in microvascular function in prediabetes was $\sim 1 / 2$ to $1 / 4$ of that in T2D (Table 2.2). Our interpretation of a graded decline in microvascular function with worsening glucose tolerance is supported by the significant associations of higher levels of $\mathrm{HbA1C}$ and FPG with attenuated retinal and skin microvascular responses (Table 2.3 and Figure 2.4), which suggests that microvascular dysfunction is closely related to hyperglycemia. Thus, we attribute the fact that direct comparison of microvascular function between the NGM and prediabetes groups was not statistically significant in adjusted analyses to a type 2 statistical error, as power in such betweengroup comparisons is reduced compared to analyses of trends and of continuous variables such as $\mathrm{HbA1C}$.

Impairments in both flicker light-induced retinal arteriolar dilation (which is nitric oxide-dependent ${ }^{29,30}$ ) and heat-induced skin hyperemia (which depends on nitric oxide and endothelium-derived hyperpolarizing factors ${ }^{31,32}$ ) are likely to reflect microvascular endothelial dysfunction ${ }^{29,32}$, possibly in conjunction with neuronal dysfunction ${ }^{13,17,31,33}$. The mechanism that may underlie the association between hyperglycemia and microvascular endothelial dysfunction may be bidirectional, i.e. microvascular endothelial dysfunction may act as both consequence and cause of hyperglycemia ${ }^{2}$. On the one hand, hyperglycemia can impair endothelial function through formation of advanced glycation end products, intraendothelial accumulation of glucose, and increased oxidative stress ${ }^{34}$. On the other hand, microvascular endothelial dysfunction can cause or aggravate hyperglycemia, and lead to (pre)diabetes ${ }^{35,36}$, both by impairing the timely access of glucose and insulin to their target tissues ${ }^{37}$ as well as by impairing insulin secretion $^{38}$. Consequently, a vicious circle may exist between hyperglycemia and microvascular endothelial dysfunction.

Strengths of our study include its population-based design with oversampling of individuals with $\mathrm{T} 2 \mathrm{D}$, which enables an accurate comparison of individuals without and with T2D; the use of an OGTT and HbA1c levels to characterize glucose metabolism; the use of two, independent, methods to directly assess microvascular function, instead of relying on indirect biomarkers ${ }^{36,39}$ or estimates of microvascular structure ${ }^{35}$; the extensive assessment of potential confounders, including 24-h ambulatory blood pressure, and the broad array of additional analyses, which all gave consistent results.

Our study had some limitations. First, the data were cross-sectional; therefore, we cannot exclude reverse causality. However, from the association between diabetes and microangiopathy it follows that there is a strong a priori likelihood that hyperglycemia 
can cause microvascular dysfunction. On the other hand, it appears likely that microvascular dysfunction can cause hyperglycemia ${ }^{37}$, and we in fact hypothesize that the association is bidirectional ${ }^{36,40}$. Second, we measured microvascular dysfunction in retina and skin, and infer that findings can be generalized to other microvascular beds ${ }^{41}$, but this inference needs to be formally tested. Third, we studied Caucasian individuals aged $40-75$ years who were relatively intensively treated with regard to vascular and metabolic risk factors; therefore extrapolation to other groups will need further study. Fourth, our fully adjusted model may have been overadjusted ${ }^{42}$, as diabetic retinopathy and nephropathy have a microvascular origin. However, qualitatively similar results were obtained when we excluded T2D participants with clinically apparent diabetic microangiopathy. Fifth, although we adjusted for many potential confounders, we cannot fully exclude residual confounding by variables not included in these analyses (e.g. dietary habits). Sixth, a single OGTT may result in misclassification of long-term glucose tolerance status ${ }^{43}$. Therefore, what may be considered prediabetes may be unrecognized T2D (which would overestimate microvascular dysfunction in prediabetes), or vice versa, and what may be considered NGM may be unrecognized prediabetes, or vice versa (all of which would underestimate microvascular dysfunction in prediabetes). When group size of the NGM, prediabetes, and T2D groups and misclassification estimates $^{43}$ are taken into account, the net result of these opposing effects is likely to be underestimation of microvascular dysfunction in the prediabetes group.

In summary, we showed, in a population-based study, that prediabetes and T2D, and continuous measures of hyperglycemia, are associated with impaired microvascular function in retina and skin, independently of major cardiovascular risk factors and clinically defined diabetic retinopathy and nephropathy. These findings, therefore, support the concept that generalized microvascular dysfunction precedes the clinical diagnosis of T2D and may contribute to the development of microvascular complications in T2D and prediabetes, such as retinopathy, nephropathy, neuropathy, heart failure, stroke, and cognitive decline. These findings suggest that both early hyperglycemia and microvascular dysfunction should be considered as potential targets of intervention. 


\section{References}

1. Guariguata L, Whiting DR, Hambleton I, Beagley J, Linnenkamp U and Shaw JE. Global estimates of diabetes prevalence for 2013 and projections for 2035. Diabetes Res Clin Pract. 2014;103:137-49.

2. van Sloten TT, Henry RM, Dekker JM, Nijpels G, Unger T, Schram MT and Stehouwer CD. Endothelial dysfunction plays a key role in increasing cardiovascular risk in type 2 diabetes: the Hoorn study. Hypertension. 2014;64:1299-305.

3. Forouhi NG and Wareham NJ. Epidemiology of diabetes. Medicine. 2014;42:698-702.

4. Lee JF, Barrett-O'Keefe Z, Garten RS, Nelson AD, Ryan JJ, Nativi JN, Richardson RS and Wray DW. Evidence of microvascular dysfunction in heart failure with preserved ejection fraction. Heart. 2016;102:278-84.

5. Buysschaert M, Medina JL, Bergman M, Shah A and Lonier J. Prediabetes and associated disorders. Endocrine. 2015;48:371-93.

6. Mostaza JM, Lahoz C, Salinero-Fort MA, de Burgos-Lunar C, Laguna F, Estirado E, Garcia-Iglesias F, Gonzalez-Alegre T, Cornejo-Del-Rio V, Sabin C, Lopez S and Group S-. Carotid atherosclerosis severity in relation to glycemic status: a cross-sectional population study. Atherosclerosis. 2015;242:377-82.

7. Schram MT, Henry RM, van Dijk RA, Kostense PJ, Dekker JM, Nijpels G, Heine RJ, Bouter LM, Westerhof N and Stehouwer CD. Increased central artery stiffness in impaired glucose metabolism and type 2 diabetes: the Hoorn Study. Hypertension. 2004;43:176-81.

8. Su Y, Liu XM, Sun YM, Wang YY, Luan Y and Wu Y. Endothelial dysfunction in impaired fasting glycemia, impaired glucose tolerance, and type 2 diabetes mellitus. Am J Cardiol. 2008;102:497-8.

9. Wong MS, Gu K, Heng D, Chew SK, Chew LS and Tai ES. The Singapore impaired glucose tolerance followup study: does the ticking clock go backward as well as forward? Diabetes Care. 2003;26:3024-30.

10. Ford ES, Zhao G and Li C. Pre-diabetes and the risk for cardiovascular disease: a systematic review of the evidence. J Am Coll Cardiol. 2010;55:1310-7.

11. Keymel S, Heinen Y, Balzer J, Rassaf T, Kelm M, Lauer T and Heiss C. Characterization of macro- and microvascular function and structure in patients with type 2 diabetes mellitus. Am J Cardiovasc Dis. 2011;1:68-75.

12. Caballero AE, Arora S, Saouaf R, Lim SC, Smakowski P, Park JY, King GL, LoGerfo FW, Horton ES and Veves A. Microvascular and macrovascular reactivity is reduced in subjects at risk for type 2 diabetes. Diabetes. 1999;48:1856-62.

13. Garhofer G, Zawinka C, Resch H, Kothy $P$, Schmetterer L and Dorner GT. Reduced response of retinal vessel diameters to flicker stimulation in patients with diabetes. Br J Ophthalmol. 2004;88:887-91.

14. Nguyen TT, Kreis AJ, Kawasaki R, Wang JJ, Seifert BU, Vilser W, Nagel E and Wong TY. Reproducibility of the retinal vascular response to flicker light in Asians. Curr Eye Res. 2009;34:1082-8.

15. Agarwal SC, Allen J, Murray A and Purcell IF. Comparative reproducibility of dermal microvascular blood flow changes in response to acetylcholine iontophoresis, hyperthermia and reactive hyperaemia. Physiol Meas. 2010;31:1-11.

16. Cheung $\mathrm{CY}$, Ikram MK, Klein R and Wong TY. The clinical implications of recent studies on the structure and function of the retinal microvasculature in diabetes. Diabetologia. 2015;58:871-85.

17. Minson CT, Berry LT and Joyner MJ. Nitric oxide and neurally mediated regulation of skin blood flow during local heating. J App/ Physiol (1985). 2001;91:1619-26.

18. Schram MT, Sep SJ, van der Kallen CJ, Dagnelie PC, Koster A, Schaper NC, Henry RM and Stehouwer CD. The Maastricht Study: an extensive phenotyping study on determinants of type 2 diabetes, its complications and its comorbidities. Eur J Epidemiol. 2014;29:439-51.

19. Garhofer G, Resch H, Sacu S, Weigert G, SchmidI D, Lasta M and Schmetterer L. Effect of regular smoking on flicker induced retinal vasodilatation in healthy subjects. Microvasc Res. 2011;82:351-5.

20. Jonk AM, Houben AJ, Schaper NC, de Leeuw PW, Serne EH, Smulders YM and Stehouwer CD. Mealrelated increases in microvascular vasomotion are impaired in obese individuals: a potential mechanism in the pathogenesis of obesity-related insulin resistance. Diabetes Care. 2011;34 Suppl 2:S342-8.

21. Nagel $\mathrm{E}$ and Vilser W. Flicker observation light induces diameter response in retinal arterioles: a clinical methodological study. Br J Ophthalmol. 2004;88:54-6. 
22. Nagel E, Vilser W, Fink A and Riemer T. Variance of retinal vessel diameter response to flicker light. A methodical clinical study. Ophthalmologe. 2006;103:114-9.

23. Muris DM, Houben AJ, Kroon AA, Henry RM, van der Kallen CJ, Sep SJ, Koster A, Dagnelie PC, Schram MT and Stehouwer CD. Age, waist circumference, and blood pressure are associated with skin microvascular flow motion: the Maastricht Study. J Hypertens. 2014;32:2439-49.

24. Roustit $\mathrm{M}$ and Cracowski JL. Assessment of endothelial and neurovascular function in human skin microcirculation. Trends Pharmacol Sci. 2013;34:373-84.

25. Inker LA, Schmid CH, Tighiouart H, Eckfeldt JH, Feldman HI, Greene T, Kusek JW, Manzi J, Van Lente F, Zhang YL, Coresh J, Levey AS and Investigators C-E. Estimating glomerular filtration rate from serum creatinine and cystatin C. N Engl J Med. 2012;367:20-9.

26. Nguyen TT, Kawasaki R, Wang JJ, Kreis AJ, Shaw J, Vilser W and Wong TY. Flicker light-induced retinal vasodilation in diabetes and diabetic retinopathy. Diabetes Care. 2009;32:2075-80.

27. Lott ME, Slocomb JE, Shivkumar V, Smith B, Quillen D, Gabbay RA, Gardner TW and Bettermann K. Impaired retinal vasodilator responses in prediabetes and type 2 diabetes. Acta Ophthalmol. 2013;91:e462-9.

28. Sokolnicki LA, Roberts SK, Wilkins BW, Basu A and Charkoudian N. Contribution of nitric oxide to cutaneous microvascular dilation in individuals with type 2 diabetes mellitus. Am J Physiol Endocrinol Metab. 2007;292:E314-8.

29. Lim M, Sasongko MB, Ikram MK, Lamoureux E, Wang JJ, Wong TY and Cheung CY. Systemic associations of dynamic retinal vessel analysis: a review of current literature. Microcirculation. 2013;20:257-68.

30. Dorner GT, Garhofer G, Kiss B, Polska E, Polak K, Riva CE and Schmetterer L. Nitric oxide regulates retinal vascular tone in humans. Am J Physiol Heart Circ Physiol. 2003;285:H631-6.

31. Choi PJ, Brunt VE, Fujii N and Minson CT. New approach to measure cutaneous microvascular function: an improved test of NO-mediated vasodilation by thermal hyperemia. J Appl Physiol (1985). 2014;117:277-83

32. Kellogg DL, Jr., Liu Y, Kosiba IF and O'Donnell D. Role of nitric oxide in the vascular effects of local warming of the skin in humans. J Appl Physiol (1985). 1999;86:1185-90.

33. Kern TS and Barber AJ. Retinal ganglion cells in diabetes. J Physiol. 2008;586:4401-8.

34. Chilelli NC, Burlina S and Lapolla A. AGEs, rather than hyperglycemia, are responsible for microvascular complications in diabetes: a "glycoxidation-centric" point of view. Nutr Metab Cardiovasc Dis. 2013;23:913-9.

35. Sabanayagam C, Lye WK, Klein R, Klein BE, Cotch MF, Wang JJ, Mitchell P, Shaw JE, Selvin E, Sharrett AR and Wong TY. Retinal microvascular calibre and risk of diabetes mellitus: a systematic review and participant-level meta-analysis. Diabetologia. 2015;58:2476-85.

36. Muris DM, Houben AJ, Schram MT and Stehouwer CD. Microvascular dysfunction is associated with a higher incidence of type 2 diabetes mellitus: a systematic review and meta-analysis. Arterioscler Thromb Vasc Biol. 2012;32:3082-94.

37. Jonk AM, Houben AJ, de Jongh RT, Serne EH, Schaper NC and Stehouwer CD. Microvascular dysfunction in obesity: a potential mechanism in the pathogenesis of obesity-associated insulin resistance and hypertension. Physiology. 2007;22:252-60.

38. Hashimoto S, Kubota N, Sato H, Sasaki M, Takamoto I, Kubota T, Nakaya K, Noda M, Ueki K and Kadowaki T. Insulin receptor substrate-2 (Irs2) in endothelial cells plays a crucial role in insulin secretion. Diabetes. 2015;64:876-86.

39. Meigs JB, Hu FB, Rifai $\mathrm{N}$ and Manson JE. Biomarkers of endothelial dysfunction and risk of type 2 diabetes mellitus. JAMA. 2004;291:1978-86.

40. Muris DM, Houben AJ, Schram MT and Stehouwer CD. Microvascular dysfunction: an emerging pathway in the pathogenesis of obesity-related insulin resistance. Rev Endocr Metab Dis. 2013;14:29-38.

41. Prior JO, Quinones MJ, Hernandez-Pampaloni M, Facta AD, Schindler TH, Sayre JW, Hsueh WA and Schelbert HR. Coronary circulatory dysfunction in insulin resistance, impaired glucose tolerance, and type 2 diabetes mellitus. Circulation. 2005;111:2291-8.

42. Schisterman EF, Cole SR and Platt RW. Overadjustment bias and unnecessary adjustment in epidemiologic studies. Epidemiology. 2009;20:488-95. 


\section{Chapter 2}

43. Mooy JM, Grootenhuis PA, de Vries H, Kostense PJ, Popp-Snijders C, Bouter LM and Heine RJ. Intraindividual variation of glucose, specific insulin and proinsulin concentrations measured by two oral glucose tolerance tests in a general Caucasian population: the Hoorn Study. Diabetologia. 1996;39: 298-305. 


\section{Supplemental Material to chapter 2}

\section{Supplemental Methods}

\section{Statistical analysis}

Differences in general characteristics among individuals in the study populations and individuals excluded due to missing values were compared by Analyses of Variance (ANOVA) for continuous variables and $\chi^{2}$-test for categorical variables.

\section{Appendix 2.1}

In 596 individuals heat-induced skin hyperemia measurements were recorded between 20-25 minutes. These data were extrapolated to 25 minutes using the last minute average as reference; with a weighted correction factor of 1.017. This correction factor is the ratio of the increase in average perfusion units in the 20-25 interval as compared to the average perfusion units in the 19-20 interval.

\section{Supplemental Tables}




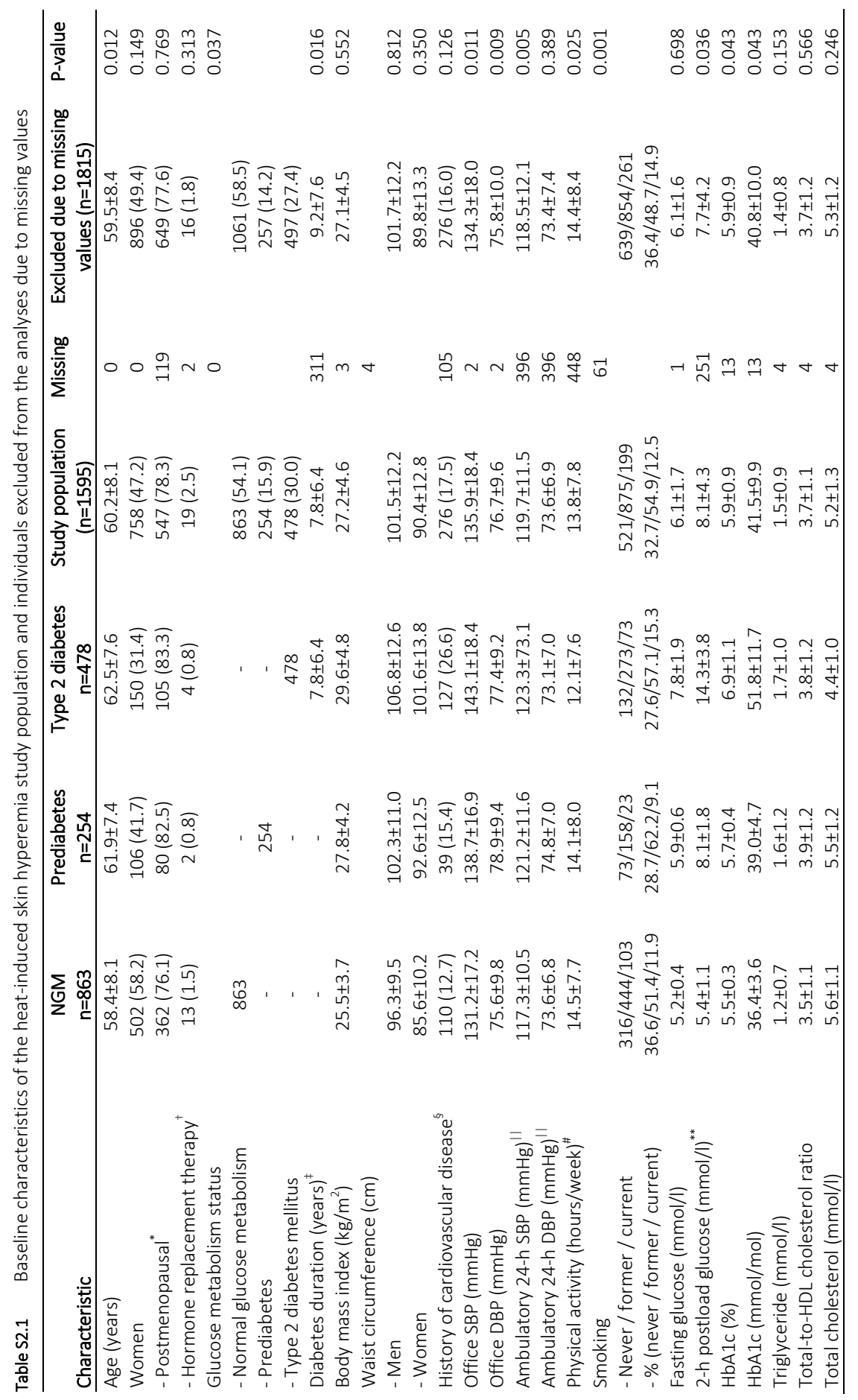




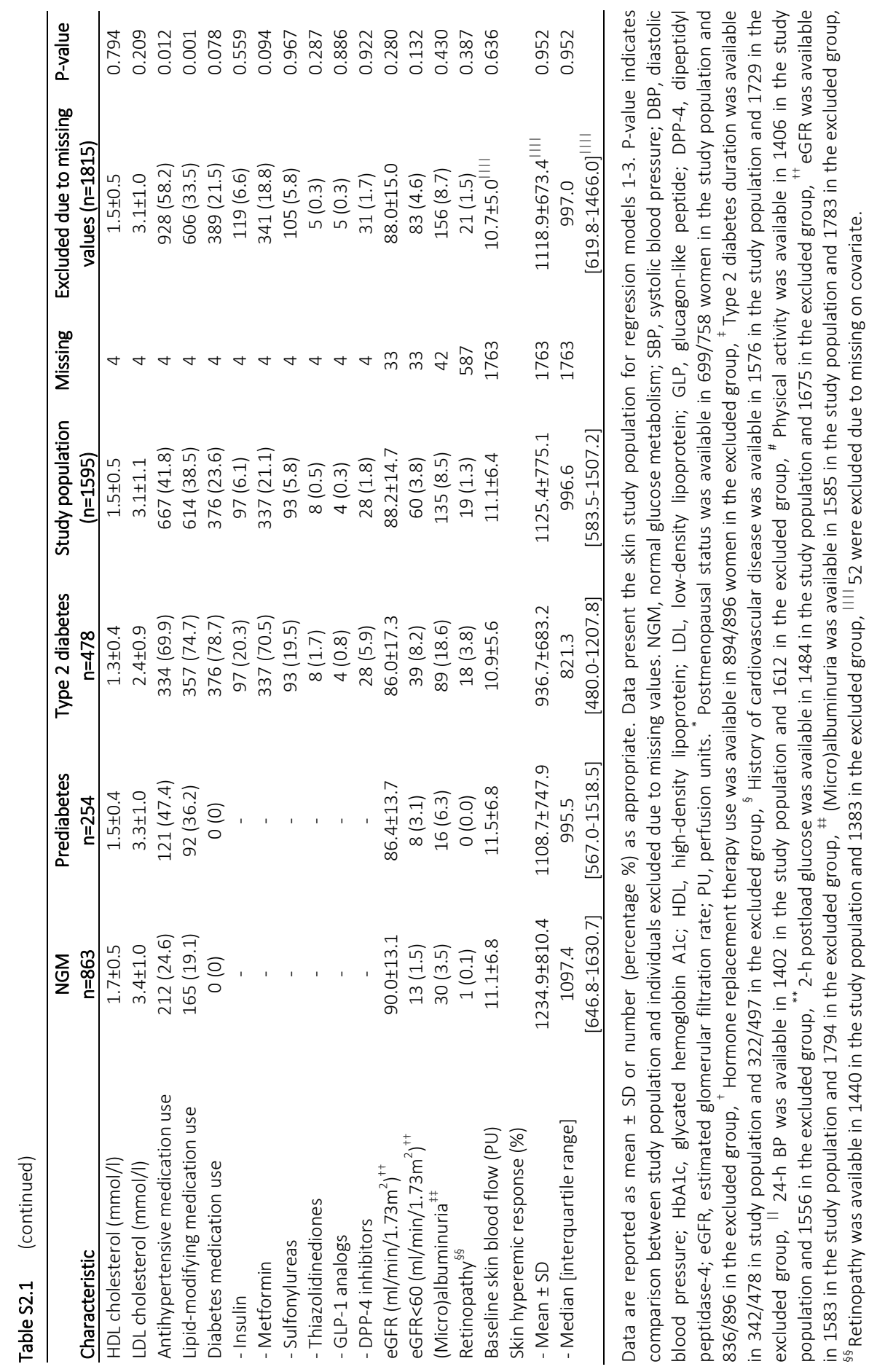


Table S2.2 Baseline characteristics of the retinal arteriolar reactivity study population and individuals excluded from the analyses due to missing values

\begin{tabular}{|c|c|c|c|c|}
\hline Characteristic & $\begin{array}{c}\text { Retinal reactivity study } \\
\text { population }(n=2213)\end{array}$ & Missing & $\begin{array}{c}\text { Excluded due to missing } \\
\text { values }(n=1197)\end{array}$ & P-value \\
\hline Age (years) & $59.7 \pm 8.2$ & 0 & $60.0 \pm 8.3$ & 0.202 \\
\hline Women & 1083 (48.9) & 0 & $571(47.7)$ & 0.257 \\
\hline - Postmenopausal ${ }^{*}$ & $811(78.1)$ & 119 & $385(77.6)$ & 0.848 \\
\hline - Hormone replacement therapy ${ }^{\dagger}$ & $18(1.7)$ & 2 & $17(3.0)$ & 0.075 \\
\hline Glucose metabolism status & & 0 & & 0.167 \\
\hline - Normal glucose metabolism & $1269(57.3)$ & & $655(54.7)$ & \\
\hline - Prediabetes & $335(15.1)$ & & $176(14.7)$ & \\
\hline - Type 2 diabetes mellitus & $609(27.5)$ & & $366(30.6)$ & \\
\hline Diabetes duration (years) $^{\ddagger}$ & $8.2 \pm 7.0$ & 311 & $9.0 \pm 7.1$ & 0.189 \\
\hline Body mass index $\left(\mathrm{kg} / \mathrm{m}^{2}\right)$ & $26.9 \pm 4.5$ & 3 & $27.5 \pm 4.7$ & 0.001 \\
\hline Waist circumference $(\mathrm{cm})$ & & 4 & & \\
\hline - Men & $101.2 \pm 12.0$ & & $102.2 \pm 12.3$ & 0.096 \\
\hline - Women & $89.2 \pm 12.6$ & & $91.7 \pm 13.7$ & $<0.001$ \\
\hline History of cardiovascular disease ${ }^{\S}$ & $339(15.5)$ & 105 & $213(19.0)$ & 0.007 \\
\hline Office SBP (mmHg) & $134.9 \pm 18.0$ & 2 & $135.2 \pm 18.5$ & 0.671 \\
\hline Office DBP (mmHg) & $76.4 \pm 9.9$ & 2 & $75.9 \pm 9.7$ & 0.183 \\
\hline Ambulatory 24-h SBP $(\mathrm{mmHg})^{\|}$ & $119.1 \pm 11.5$ & 396 & $118.9 \pm 12.4$ & 0.698 \\
\hline Ambulatory 24-h DBP (mmHg) ${ }^{\prime \prime}$ & $73.5 \pm 7.2$ & 396 & $73.5 \pm 7.2$ & 0.941 \\
\hline Physical activity (hours/week) & $14.0 \pm 8.0$ & 448 & $14.4 \pm 8.4$ & 0.143 \\
\hline Smoking & & 61 & & 0.006 \\
\hline - Never / former / current & $780 / 1159 / 274$ & & $380 / 570 / 186$ & \\
\hline - \% (never / former / current) & $35.2 / 52.4 / 12.4$ & & $33.5 / 50.2 / 16.4$ & \\
\hline Fasting glucose (mmol/l) & $6.0 \pm 1.7$ & 1 & $6.1 \pm 1.7$ & 0.088 \\
\hline 2-h postload glucose $(\mathrm{mmol} / \mathrm{l})^{* *}$ & $7.9 \pm 4.2$ & 251 & $7.9 \pm 4.3$ & 0.564 \\
\hline $\mathrm{HbA1c}(\%)$ & $5.9 \pm 0.9$ & 13 & $6.0 \pm 0.9$ & $<0.001$ \\
\hline $\mathrm{HbA} 1 \mathrm{c}(\mathrm{mmol} / \mathrm{mol})$ & $40.5 \pm 9.8$ & 13 & $42.1 \pm 10.3$ & $<0.001$ \\
\hline Triglyceride (mmol/l) & $1.4 \pm 0.8$ & 4 & $1.4 \pm 0.9$ & 0.651 \\
\hline Total-to-HDL cholesterol ratio & $3.6 \pm 1.1$ & 4 & $3.8 \pm 1.2$ & $<0.001$ \\
\hline Total cholesterol (mmol/l) & $5.2 \pm 1.2$ & 4 & $5.2 \pm 1.1$ & 0.182 \\
\hline HDL cholesterol (mmol/l) & $1.6 \pm 0.5$ & 4 & $1.5 \pm 0.4$ & $<0.001$ \\
\hline LDL cholesterol (mmol/l) & $3.1 \pm 1.0$ & 4 & $3.1 \pm 1.0$ & 0.131 \\
\hline Antihypertensive medication use & $845(38.2)$ & 4 & $510(42.7)$ & 0.005 \\
\hline Lipid-modifying medication use & $774(35.0)$ & 4 & $446(37.4)$ & 0.087 \\
\hline Diabetes medication use & $476(21.5)$ & 4 & $289(24.2)$ & 0.039 \\
\hline - Insulin & $121(5.5)$ & 4 & $95(8.0)$ & 0.004 \\
\hline - Metformin & $423(19.1)$ & 4 & $255(21.4)$ & 0.115 \\
\hline - Sulfonylureas & $119(5.4)$ & 4 & $79(6.6)$ & 0.139 \\
\hline - Thiazolidinediones & $6(0.3)$ & 4 & $7(0.6)$ & 0.154 \\
\hline - GLP-1 analogs & $3(0.1)$ & 4 & $6(0.5)$ & 0.046 \\
\hline - DPP-4 inhibitors & 43 (1.9) & 4 & $16(1.3)$ & 0.199 \\
\hline $\operatorname{eGFR}\left(\mathrm{ml} / \mathrm{min} / 1.73 \mathrm{~m}^{2}\right)^{\dagger+}$ & $88.3 \pm 14.6$ & 33 & $87.7 \pm 15.3$ & 0.280 \\
\hline eGFR<60 $\left(\mathrm{ml} / \mathrm{min} / 1.73 \mathrm{~m}^{2}\right)^{++}$ & $92(4.2)$ & 33 & $51(4.3)$ & 0.453 \\
\hline (Micro)albuminuria ${ }^{\ddagger \ddagger}$ & $179(8.1)$ & 42 & $112(9.6)$ & 0.089 \\
\hline Retinopathy ${ }^{\S \S}$ & $27(1.3)$ & 587 & $13(1.9)$ & 0.137 \\
\hline Baseline arteriolar diameter (MU) & $115.5 \pm 15.7$ & 1149 & $115.5 \pm 14.4^{\prime \prime \prime I}$ & 0.970 \\
\hline \multicolumn{5}{|l|}{ Arteriolar average dilation (\%) } \\
\hline- Mean \pm SD & $3.0 \pm 2.8$ & 1149 & $2.0 \pm 2.4^{1111}$ & 0.011 \\
\hline - Median [interquartile range] & $2.6[0.8-5.0]$ & 1149 & $1.5[0.1-3.7]^{|1| \mid}$ & 0.011 \\
\hline
\end{tabular}


Data are reported as mean \pm SD or number (percentage \%) as appropriate. P-value indicates comparison between study population and individuals excluded due to missing values. SBP, systolic blood pressure; DBP, diastolic blood pressure; HbA1c, glycated hemoglobin A1c; HDL, high-density lipoprotein; LDL, low-density lipoprotein; GLP, glucagon-like peptide; DPP-4, dipeptidyl peptidase-4; eGFR, estimated glomerular filtration rate; $\mathrm{MU}$, measurement units. ${ }^{*}$ Postmenopausal status was available in 1039/1083 women in study population and 496/571 in the excluded group, ${ }^{\dagger}$ Hormone replacement therapy use was available 569/571 women in the excluded group, ${ }^{\ddagger}$ Type 2 diabetes duration was available in 420/609 in study population and 244/366 in the excluded group, ${ }^{\S}$ History of cardiovascular disease was available in 2184 in the study population and 1121 in the excluded group, " 24-h BP was available in 1962 in the study population and 1052 in the excluded group, \# Physical activity was available in 1974 in the study population and 988 in the excluded group, ${ }^{* *}$ 2-h postload glucose was available in 2075 in the study population and 1084 in the excluded group, ${ }^{+\dagger}$ eGFR was available in 2199 in the study population and 1178 in the excluded group, ${ }^{\ddagger \ddagger}$ (Micro)albuminuria was available in 2199 in the study population and 1169 in the excluded group, ${ }^{\S \S}$ Retinopathy was available in 2148 in the study population and 675 in the excluded group, "III 48 were excluded due to missing on covariate.

Table S2.3 Multivariable-adjusted differences in flicker light-induced retinal arteriolar \%-dilation and heatinduced skin \%-hyperemia among individuals with normal glucose metabolism (NGM), prediabetes, and type 2 diabetes with replacement of office by 24-h systolic ambulatory blood pressure in regression models

\begin{tabular}{lccc}
\hline Characteristic & Prediabetes* & Type 2 diabetes* & P for trend \\
\hline Retinal arteriolar average dilation (\%) & $\mathrm{B}(95 \% \mathrm{Cl})$ & $\mathrm{B}(95 \% \mathrm{Cl})$ & \\
Crude & $-0.36(-0.72 ;-0.05)$ & $-1.05(-1.34 ;-0.77)$ & $<0.001$ \\
Model 2 & $-0.22(-0.58 ; 0.14)$ & $-0.86(-1.18 ;-0.60)$ & $<0.001$ \\
Model 3 & $-0.16(-0.53 ; 0.21)$ & $-0.65(-1.02 ;-0.29)$ & 0.001 \\
Model 4 & $-0.18(-0.56 ; 0.20)$ & $-0.59(-0.97 ;-0.22)$ & 0.003 \\
Retinal arteriolar average dilation (SD) & stB $(95 \% \mathrm{Cl})$ & stB $(95 \% \mathrm{Cl})$ & \\
Crude & $-0.05(-0.09 ;-0.00)$ & $-0.17(-0.21 ;-0.12)$ & $<0.001$ \\
Model 2 & $-0.03(-0.07 ; 0.02)$ & $-0.14(-0.18 ;-0.09)$ & $<0.001$ \\
Model 3 & $-0.02(-0.07 ; 0.03)$ & $-0.10(-0.16 ;-0.05)$ & 0.001 \\
Model 4 & $-0.02(-0.07 ; 0.03)$ & $-0.10(-0.16 ;-0.03)$ & 0.003 \\
\hline Heat-induced skin hyperemia (\%) & $\mathrm{B}(95 \% \mathrm{Cl})$ & $\mathrm{B}(95 \% \mathrm{Cl})$ & \\
Crude & $-134(-249 ;-19)$ & $-305(-397 ;-213)$ & $<0.001$ \\
Model 2 & $-59(-175 ; 54)$ & $-191(-285 ;-96)$ & $<0.001$ \\
Model 3 & $-57(-174 ; 60)$ & $-183(-294 ;-72)$ & 0.002 \\
Model 4 & $-40(-166 ; 85)$ & $-163(-283 ;-44)$ & 0.013 \\
Heat-induced skin hyperemia (SD) & stB $(95 \% \mathrm{Cl})$ & stB $(95 \% \mathrm{Cl})$ & \\
Crude & $-0.06(-0.12 ;-0.01)$ & $-0.18(-0.23 ;-0.13)$ & $<0.001$ \\
Model 2 & $-0.03(-0.08 ; 0.03)$ & $-0.11(-0.17 ;-0.06)$ & $<0.001$ \\
Model 3 & $-0.03(-0.08 ; 0.03)$ & $-0.11(-0.17 ;-0.04)$ & 0.002 \\
Model 4 & $-0.02(-0.08 ; 0.04)$ & $-0.10(-0.17 ;-0.03)$ & 0.013 \\
\hline
\end{tabular}

Regression coefficients $(B)$ indicate the mean difference $(95 \% \mathrm{Cl}$ ) in retinal and skin measures (in \%) with NGM as reference. Standardized regression coefficients (stB) and $95 \% \mathrm{Cl}$ represent the change in retinal and skin measures (in SD) as compared to NGM. SD, standard deviation; $\mathrm{Cl}$, confidence interval.

Model 2: adjusted for age and sex. Model 3: additionally adjusted for body mass index, triglyceride levels, total-to-HDL-cholesterol ratio, smoking status, 24-h systolic blood pressure, and use of antihypertensive and/or lipid-modifying drugs. Model 4: additionally adjusted for history of cardiovascular disease, retinopathy, eGFR, and urinary albumin excretion. ${ }^{*} 24-h$ ambulatory blood pressure was available in $n=1962$ of the retinal reactivity population (291 individuals with prediabetes and 544 with type 2 diabetes) and in $n=1402$ of the heat-induced skin hyperemia population (221 individuals with prediabetes and 420 with type 2 diabetes). 
Table S2.4 Multivariable-adjusted differences in flicker light-induced retinal arteriolar \%-dilation and heatinduced skin \%-hyperemia among individuals with normal glucose metabolism (NGM), prediabetes, and type 2 diabetes adjusted for physical activity

\begin{tabular}{|c|c|c|c|}
\hline Characteristic & Prediabetes* & Type 2 diabetes* & P for trend \\
\hline Retinal arteriolar average dilation (\%) & $\mathrm{B}(95 \% \mathrm{Cl})$ & $\mathrm{B}(95 \% \mathrm{Cl})$ & \\
\hline Crude & $-0.39(-0.74 ;-0.05)$ & $-1.06(-1.35 ;-0.78)$ & $<0.001$ \\
\hline Model 2 & $-0.27(-0.62 ; 0.08)$ & $-0.91(-1.21 ;-0.62)$ & $<0.001$ \\
\hline Model 3 & $-0.19(-0.55 ; 0.17)$ & $0.66(-1.03 ;-0.30)$ & 0.001 \\
\hline Model 4 & $-0.21(-0.58 ; 0.16)$ & $-0.62(-0.99 ;-0.24)$ & 0.002 \\
\hline Retinal arteriolar average dilation (SD) & stB $(95 \% \mathrm{Cl})$ & stB $(95 \% \mathrm{Cl})$ & \\
\hline Crude & $-0.05(-0.10 ;-0.01)$ & $-0.17(-0.21 ;-0.12)$ & $<0.001$ \\
\hline Model 2 & $-0.04(-0.08 ; 0.01)$ & $-0.15(-0.19 ;-0.10)$ & $<0.001$ \\
\hline Model 3 & $-0.02(-0.07 ; 0.02)$ & $-0.11(-0.16 ;-0.05)$ & 0.001 \\
\hline Model 4 & $-0.03(-0.08 ; 0.02)$ & $-0.10(-0.16 ;-0.04)$ & 0.002 \\
\hline Heat-induced skin hyperemia (\%) & $\mathrm{B}(95 \% \mathrm{Cl})$ & $\mathrm{B}(95 \% \mathrm{Cl})$ & \\
\hline Crude & $-144(-257 ;-31)$ & $-285(-378 ;-192)$ & $<0.001$ \\
\hline Model 2 & $-70(-183 ; 42)$ & $-174(-269 ;-79)$ & $<0.001$ \\
\hline Model 3 & $-79(-195 ; 37)$ & $-182(-295 ;-68)$ & $<0.001$ \\
\hline Model 4 & $-71(-195 ; 53)$ & $-155(-276 ;-34)$ & $<0.001$ \\
\hline Heat-induced skin hyperemia (SD) & stB $(95 \% \mathrm{Cl})$ & stB $(95 \% \mathrm{Cl})$ & \\
\hline Crude & $-0.07(-0.12 ;-0.02)$ & $-0.17(-0.22 ;-0.11)$ & $<0.001$ \\
\hline Model 2 & $-0.03(-0.09 ; 0.02)$ & $-0.10(-0.16 ;-0.05)$ & $<0.001$ \\
\hline Model 3 & $-0.04(-0.09 ; 0.02)$ & $-0.11(-0.17 ;-0.04)$ & $<0.001$ \\
\hline Model 4 & $-0.03(-0.09 ; 0.03)$ & $-0.09(-0.16 ;-0.02)$ & $<0.001$ \\
\hline
\end{tabular}

Regression coefficients $(B)$ indicate the mean difference $(95 \% \mathrm{Cl}$ ) in retinal and skin measures (in \%) with NGM as reference. Standardized regression coefficients $(\mathrm{stB})$ and $95 \% \mathrm{Cl}$ represent the change in retinal and skin measures (in SD) as compared to NGM. SD, standard deviation; $\mathrm{Cl}$, confidence interval.

Model 2: adjusted for age and sex. Model 3: additionally adjusted for body mass index, triglyceride levels, total-to-HDL-cholesterol ratio, smoking status, systolic blood pressure, use of antihypertensive and/or lipidmodifying drugs, and physical activity. Model 4: additionally adjusted for history of cardiovascular disease, retinopathy, eGFR, and urinary albumin excretion. * Physical activity data were available in $n=1974$ of the retinal reactivity population (304 individuals with prediabetes and 525 with type 2 diabetes) and in $n=1406$ of the heat-induced skin hyperemia population (229 individuals with prediabetes and 396 with type 2 diabetes). 
Table S2.5 Multivariable-adjusted differences in flicker light-induced retinal arteriolar \%-dilation and heatinduced skin \%-hyperemia among individuals with normal glucose metabolism (NGM), prediabetes, and type 2 diabetes adjusted for antihypertensive treatment

\begin{tabular}{|c|c|c|c|}
\hline Characteristic & Prediabetes & Type 2 diabetes & P for trend \\
\hline Retinal arteriolar average dilation (\%) & $\mathrm{B}(95 \% \mathrm{Cl})$ & $\mathrm{B}(95 \% \mathrm{Cl})$ & \\
\hline Crude & $-0.39(-0.73 ;-0.06)$ & $-1.07(-1.34 ;-0.80)$ & $<0.001$ \\
\hline Model 2 & $-0.27(-0.60 ; 0.07)$ & $-0.90(-1.18 ;-0.62)$ & $<0.001$ \\
\hline Model 2a & $-0.22(-0.56 ; 0.12)$ & $-0.78(-1.08 ;-0.47)$ & $<0.001$ \\
\hline Model 2b & $-0.26(-0.59 ; 0.08)$ & $-0.89(-1.17 ;-0.61)$ & $<0.001$ \\
\hline Model 2c & $-0.22(-0.56 ; 0.12)$ & $-0.78(-1.09 ;-0.47)$ & $<0.001$ \\
\hline Model 3 & $-0.19(-0.53 ; 0.16)$ & $-0.66(-1.01 ;-0.32)$ & $<0.001$ \\
\hline Model 4 & $-0.20(-0.56 ; 0.15)$ & $-0.62(-0.98 ;-0.26)$ & 0.001 \\
\hline Retinal arteriolar average dilation (SD) & stB $(95 \% \mathrm{Cl})$ & stB $(95 \% \mathrm{Cl})$ & \\
\hline Crude & $-0.05(-0.09 ;-0.01)$ & $-0.17(-0.21 ;-0.13)$ & $<0.001$ \\
\hline Model 2 & $-0.03(-0.08 ; 0.01)$ & $-0.14(-0.19 ;-0.10)$ & $<0.001$ \\
\hline Model 2a & $-0.03(-0.07 ; 0.02)$ & $-0.12(-0.17 ;-0.08)$ & $<0.001$ \\
\hline Model 2b & $-0.03(-0.08 ; 0.01)$ & $-0.14(-0.19 ;-0.10)$ & $<0.001$ \\
\hline Model 2c & $-0.03(-0.07 ; 0.02)$ & $-0.12(-0.17 ;-0.08)$ & $<0.001$ \\
\hline Model 3 & $-0.02(-0.07 ; 0.02)$ & $-0.11(-0.16 ;-0.05)$ & $<0.001$ \\
\hline Model 4 & $-0.03(-0.07 ; 0.02)$ & $-0.10(-0.16 ;-0.04)$ & 0.001 \\
\hline Heat-induced skin hyperemia (\%) & $\mathrm{B}(95 \% \mathrm{Cl})$ & $\mathrm{B}(95 \% \mathrm{Cl})$ & \\
\hline Crude & $-126(-233 ;-19)$ & $-298(-384 ;-213)$ & $<0.001$ \\
\hline Model 2 & $-49(-156 ; 57)$ & $-188(-276 ;-100)$ & $<0.001$ \\
\hline Model 2a & $-49(-157 ; 59)$ & $-187(-281 ;-93)$ & $<0.001$ \\
\hline Model 2b & $-50(-157 ; 57)$ & $-188(-276 ;-100)$ & $<0.001$ \\
\hline Model 2c & $-49(-157 ; 59)$ & $-186(-281 ;-92)$ & $<0.001$ \\
\hline Model 3 & $-60(-170 ; 49)$ & $-208(-313 ;-102)$ & $<0.001$ \\
\hline Model 4 & $-48(-166 ; 69)$ & $-181(-294 ;-68)$ & 0.002 \\
\hline Heat-induced skin hyperemia (SD) & stB $(95 \% \mathrm{Cl})$ & stB $(95 \% \mathrm{Cl})$ & \\
\hline Crude & $-0.06(-0.11 ;-0.01)$ & $-0.18(-0.23 ;-0.13)$ & $<0.001$ \\
\hline Model 2 & $-0.02(-0.07 ; 0.03)$ & $-0.11(-0.16 ;-0.06)$ & $<0.001$ \\
\hline Model 2a & $-0.02(-0.07 ; 0.03)$ & $-0.11(-0.17 ;-0.06)$ & $<0.001$ \\
\hline Model 2b & $-0.02(-0.07 ; 0.03)$ & $-0.11(-0.16 ;-0.06)$ & $<0.001$ \\
\hline Model 2c & $-0.02(-0.07 ; 0.03)$ & $-0.11(-0.17 ;-0.05)$ & $<0.001$ \\
\hline Model 3 & $-0.03(-0.08 ; 0.02)$ & $-0.12(-0.19 ;-0.06)$ & $<0.001$ \\
\hline Model 4 & $-0.02(-0.08 ; 0.03)$ & $-0.11(-0.17 ;-0.04)$ & 0.002 \\
\hline
\end{tabular}

Regression coefficients $(B)$ indicate the mean difference $(95 \% \mathrm{Cl}$ ) in retinal and skin measures (in \%) with NGM as reference. Standardized regression coefficients (stB) and $95 \% \mathrm{Cl}$ represent the change in retinal and skin measures (in SD) as compared to NGM. RAAS, renin-angiotensin-aldosterone system; SD, standard deviation; $\mathrm{Cl}$, confidence interval.

Model 2: adjusted for age and sex. Model 2a: as $2+$ adjusted for antihypertensives (all types). Model 2 b: as $2+$ adjusted for non-RAAS-inhibiting antihypertensives only. Model 2c: as $2+$ adjusted separately for RAAS-inhibiting* (without or with other types of antihypertensives) and non-RAAS-inhibiting antihypertensives only. Model 3: as 2c + additionally adjusted for body mass index, triglyceride levels, total-to-HDL-cholesterol ratio, smoking status, office systolic blood pressure, and lipid-modifying drugs. Model 4: additionally adjusted for history of cardiovascular disease, retinopathy, eGFR, and urinary albumin excretion. *RAAS-inhibiting antihypertensives included angiotensin-converting-enzyme inhibitors, angiotensin receptor blockers, and renin blockers. 
Table S2.6 Multivariable-adjusted differences in flicker light-induced retinal arteriolar \%-dilation and heatinduced skin \%-hyperemia among individuals with normal glucose metabolism (NGM), prediabetes, and type 2 diabetes without microvascular complications*

\begin{tabular}{|c|c|c|c|}
\hline Characteristic & Prediabetes & Type 2 diabetes $^{\dagger}$ & $\mathrm{P}$ for trend \\
\hline Retinal arteriolar average dilation (\%) & $\mathrm{B}(95 \% \mathrm{Cl})$ & $\mathrm{B}(95 \% \mathrm{Cl})$ & \\
\hline Crude & $-0.39(-0.73 ;-0.06)$ & $-0.83(-1.13 ;-0.52)$ & $<0.001$ \\
\hline Model 2 & $-0.28(-0.62 ; 0.06)$ & $-0.71(-1.02 ;-0.39)$ & $<0.001$ \\
\hline Model 3 & $-0.22(-0.57 ; 0.13)$ & $-0.51(-0.88 ;-0.14)$ & 0.007 \\
\hline Model $4^{\ddagger}$ & $-0.20(-0.55 ; 0.16)$ & $-0.50(-0.88 ;-0.12)$ & 0.011 \\
\hline Retinal arteriolar average dilation (SD) & stB $(95 \% \mathrm{Cl})$ & stB $(95 \% \mathrm{Cl})$ & \\
\hline Crude & $-0.05(-0.10 ;-0.01)$ & $-0.12(-0.17 ;-0.08)$ & $<0.001$ \\
\hline Model 2 & $-0.04(-0.08 ; 0.01)$ & $-0.10(-0.15 ;-0.06)$ & $<0.001$ \\
\hline Model 3 & $-0.03(-0.08 ; 0.02)$ & $-0.07(-0.13 ;-0.02)$ & 0.007 \\
\hline Model $4^{\ddagger}$ & $-0.03(-0.07 ; 0.02)$ & $-0.07(-0.13 ;-0.02)$ & 0.011 \\
\hline Heat-induced skin hyperemia (\%) & $\mathrm{B}(95 \% \mathrm{Cl})$ & $\mathrm{B}(95 \% \mathrm{Cl})$ & \\
\hline Crude & $-126(-235 ;-17)$ & $-301(-400 ;-202)$ & $<0.001$ \\
\hline Model 2 & $-44(-153 ; 64)$ & $-198(-298 ;-98)$ & $<0.001$ \\
\hline Model 3 & $-63(-175 ; 49)$ & $-234(-350 ;-117)$ & $<0.001$ \\
\hline Model $4^{\ddagger}$ & $-54(-167 ; 59)$ & $-222(-340 ;-103)$ & $<0.001$ \\
\hline Heat-induced skin hyperemia (SD) & stB $(95 \% \mathrm{Cl})$ & stB $(95 \% \mathrm{Cl})$ & \\
\hline Crude & $-0.06(-0.11 ;-0.01)$ & $-0.16(-0.21 ;-0.11)$ & $<0.001$ \\
\hline Model 2 & $-0.02(-0.07 ; 0.03)$ & $-0.11(-0.16 ;-0.05)$ & $<0.001$ \\
\hline Model 3 & $-0.03(-0.09 ; 0.02)$ & $-0.12(-0.19 ;-0.06)$ & $<0.001$ \\
\hline Model $4^{\ddagger}$ & $-0.03(-0.08 ; 0.03)$ & $-0.12(-0.18 ;-0.06)$ & $<0.001$ \\
\hline
\end{tabular}

Regression coefficients $(B)$ indicate the mean difference $(95 \% \mathrm{Cl}$ ) in retinal and skin measures (in \%) with NGM as reference. Standardized regression coefficients (stB) and $95 \% \mathrm{Cl}$ represent the change in retinal and skin measures (in SD) as compared to NGM. SD, standard deviation; $\mathrm{Cl}$, confidence interval.

* Diabetic microvascular complications were defined as having retinopathy, eGFR $<60 \mathrm{ml} / \mathrm{min} / 1.73 \mathrm{~m}^{2}$, or urinary albumin excretion $>30 \mathrm{mg} / 24$ hour. ${ }^{+}$The number of individuals with type 2 diabetes in the retinal reactivity and the heat-induced skin hyperemia populations were 429 and 325 , respectively. ${ }^{\ddagger}$ Note that eGFR and urinary albumin excretion are added in the model as continuous variables. Model 2: adjusted for age and sex. Model 3: additionally adjusted for body mass index, triglyceride levels, total-to-HDL-cholesterol ratio, smoking status, 24-h systolic blood pressure, and use of antihypertensive and/or lipid-modifying drugs. Model 4: additionally adjusted for history of cardiovascular disease, eGFR, and urinary albumin excretion. 
Table S2.7 Multivariable-adjusted differences in retinal arteriolar baseline diameter, flicker light-induced retinal arteriolar \%-dilation, skin baseline blood flow, and heat-induced skin \%-hyperemia among individuals with normal glucose metabolism (NGM), prediabetes, and type 2 diabetes (T2D), after exclusion of T2D individuals using GLP-1 analogs, thiazolidinediones, or DPP-4 inhibitors

\begin{tabular}{|c|c|c|c|}
\hline Characteristic & Prediabetes* & Type 2 diabetes* & P for trend \\
\hline Retinal arteriolar average dilation (\%) & $\mathrm{B}(95 \% \mathrm{Cl})$ & $\mathrm{B}(95 \% \mathrm{Cl})$ & \\
\hline Crude & $-0.39(-0.73 ;-0.06)$ & $-1.08(-1.36 ;-0.81)$ & $<0.001$ \\
\hline Model 2 & $-0.26(-0.60 ; 0.07)$ & $-0.91(-1.20 ;-0.62)$ & $<0.001$ \\
\hline Model 3 & $-0.19(-0.53 ; 0.16)$ & $-0.66(-1.02 ;-0.31)$ & $<0.001$ \\
\hline Model 4 & $-0.20(-0.55 ; 0.16)$ & $-0.62(-1.00 ;-0.25)$ & 0.001 \\
\hline Retinal arteriolar average dilation (SD) & stB $(95 \% \mathrm{Cl})$ & stB $(95 \% \mathrm{Cl})$ & \\
\hline Crude & $-0.05(-0.10 ;-0.01)$ & $-0.18(-0.22 ;-0.13)$ & $<0.001$ \\
\hline Model 2 & $-0.04(-0.08 ; 0.01)$ & $-0.15(-0.20 ;-0.10)$ & $<0.001$ \\
\hline Model 3 & $-0.03(-0.07 ; 0.02)$ & $-0.11(-0.17 ;-0.05)$ & $<0.001$ \\
\hline Model 4 & $-0.03(-0.07 ; 0.02)$ & $-0.10(-0.16 ;-0.04)$ & 0.001 \\
\hline Heat-induced skin hyperemia (\%) & $\mathrm{B}(95 \% \mathrm{Cl})$ & $\mathrm{B}(95 \% \mathrm{Cl})$ & \\
\hline Crude & $-126(-234 ;-19)$ & $-305(-393 ;-216)$ & $<0.001$ \\
\hline Model 2 & $-49(-156 ; 58)$ & $-196(-286 ;-105)$ & $<0.001$ \\
\hline Model 3 & $-61(-171 ; 49)$ & $-213(-321 ;-105)$ & $<0.001$ \\
\hline Model 4 & $-47(-165 ; 71)$ & $-177(-293 ;-62)$ & 0.003 \\
\hline Heat-induced skin hyperemia (SD) & stB $(95 \% \mathrm{Cl})$ & stB $(95 \% \mathrm{Cl})$ & \\
\hline Crude & $-0.06(-0.11 ;-0.01)$ & $-0.18(-0.23 ;-0.13)$ & $<0.001$ \\
\hline Model 2 & $-0.02(-0.07 ; 0.03)$ & $-0.12(-0.17 ;-0.06)$ & $<0.001$ \\
\hline Model 3 & $-0.03(-0.08 ; 0.02)$ & $-0.13(-0.19 ;-0.06)$ & $<0.001$ \\
\hline Model 4 & $-0.02(-0.08 ; 0.03)$ & $-0.11(-0.17 ;-0.04)$ & 0.003 \\
\hline
\end{tabular}

Regression coefficients (B) indicate the mean difference $(95 \% \mathrm{Cl}$ ) in retinal and skin measures (in \%) with NGM as reference. Standardized regression coefficients (stB) and $95 \% \mathrm{Cl}$ represent the change in retinal and skin measures (in SD) as compared to NGM. GLP, glucagon-like peptide; DPP-4, dipeptidyl peptidase-4; SD, standard deviation; $\mathrm{Cl}$, confidence interval.

Model 2: adjusted for age and sex. Model 3: additionally adjusted for body mass index, triglyceride levels, total-to-HDL-cholesterol ratio, smoking status, systolic blood pressure, and use of antihypertensive and/or lipid-modifying drugs. Model 4: additionally adjusted for history of cardiovascular disease, retinopathy, eGFR, and urinary albumin excretion. ${ }^{*}$ The retinal reactivity population consisted of 335 individuals with prediabetes and 557 with type 2 diabetes, the heat-induced skin hyperemia population consisted of 254 individuals with prediabetes and 438 with type 2 diabetes. 
Table S2.8 Multivariable-adjusted differences in flicker light-induced retinal arteriolar \%-dilation and heatinduced skin \%-hyperemia among individuals with normal glucose metabolism (NGM), prediabetes, and type 2 diabetes adjusted for postmenopausal status and/or the use of hormone replacement therapy

\begin{tabular}{|c|c|c|c|}
\hline Characteristic & Prediabetes* & Type 2 diabetes* & P for trend \\
\hline Retinal arteriolar average dilation (\%) & $\mathrm{B}(95 \% \mathrm{Cl})$ & $\mathrm{B}(95 \% \mathrm{Cl})$ & \\
\hline Crude & $-0.42(-0.75 ;-0.08)$ & $-1.09(-1.36 ;-0.82)$ & $<0.001$ \\
\hline Model 2a & $-0.29(-0.63 ; 0.05)$ & $-0.93(-1.21 ;-0.64)$ & $<0.001$ \\
\hline Model 2b & $-0.29(-0.63 ; 0.05)$ & $-0.93(-1.22 ;-0.65)$ & $<0.001$ \\
\hline Model 2c & $-0.29(-0.63 ; 0.05)$ & $-0.93(-1.21 ;-0.65)$ & $<0.001$ \\
\hline Model 2d & $-0.29(-0.63 ; 0.04)$ & $-0.93(-1.22 ;-0.65)$ & $<0.001$ \\
\hline Model 3 & $-0.21(-0.56 ; 0.14)$ & $-0.68(-1.03 ;-0.33)$ & $<0.001$ \\
\hline Model 4 & $-0.23(-0.58 ; 0.13)$ & $-0.63(-0.99 ;-0.27)$ & 0.001 \\
\hline Retinal arteriolar average dilation (SD) & stB $(95 \% \mathrm{Cl})$ & stB $(95 \% \mathrm{Cl})$ & \\
\hline Crude & $-0.05(-0.10 ;-0.01)$ & $-0.17(-0.22 ;-0.13)$ & $<0.001$ \\
\hline Model 2a & $-0.04(-0.08 ; 0.01)$ & $-0.15(-0.19 ;-0.10)$ & $<0.001$ \\
\hline Model 2b & $-0.04(-0.08 ; 0.01)$ & $-0.15(-0.19 ;-0.10)$ & $<0.001$ \\
\hline Model 2c & $-0.04(-0.08 ; 0.01)$ & $-0.15(-0.19 ;-0.10)$ & $<0.001$ \\
\hline Model 2d & $-0.04(-0.08 ; 0.01)$ & $-0.15(-0.19 ;-0.10)$ & $<0.001$ \\
\hline Model 3 & $-0.03(-0.07 ; 0.02)$ & $-0.11(-0.16 ;-0.05)$ & $<0.001$ \\
\hline Model 4 & $-0.03(-0.08 ; 0.02)$ & $-0.10(-0.16 ;-0.04)$ & 0.001 \\
\hline Heat-induced skin hyperemia (\%) & $\mathrm{B}(95 \% \mathrm{Cl})$ & $\mathrm{B}(95 \% \mathrm{Cl})$ & \\
\hline Crude & $-139(-247 ;-31)$ & $-301(-388 ;-215)$ & $<0.001$ \\
\hline Model 2a & $-59(-167 ; 49)$ & $-187(-276 ;-97)$ & $<0.001$ \\
\hline Model 2b & $-59(-167 ; 49)$ & $-187(-277 ;-98)$ & $<0.001$ \\
\hline Model 2c & $-58(-165 ; 50)$ & $-186(-276 ;-97)$ & $<0.001$ \\
\hline Model 2d & $-58(-166 ; 50)$ & $-187(-276 ;-98)$ & $<0.001$ \\
\hline Model 3 & $-61(-171 ; 50)$ & $-191(-298 ;-85)$ & 0.001 \\
\hline Model 4 & $-57(-175 ; 61)$ & $-172(-286 ;-58)$ & 0.004 \\
\hline Heat-induced skin hyperemia (SD) & stB $(95 \% \mathrm{Cl})$ & stB $(95 \% \mathrm{Cl})$ & \\
\hline Crude & $-0.07(-0.12 ;-0.02)$ & $-0.18(-0.23 ;-0.13)$ & $<0.001$ \\
\hline Model 2a & $-0.03(-0.08 ; 0.02)$ & $-0.11(-0.16 ;-0.06)$ & $<0.001$ \\
\hline Model 2b & $-0.03(-0.08 ; 0.02)$ & $-0.11(-0.16 ;-0.06)$ & $<0.001$ \\
\hline Model 2c & $-0.03(-0.08 ; 0.02)$ & $-0.11(-0.16 ;-0.06)$ & $<0.001$ \\
\hline Model 2d & $-0.03(-0.08 ; 0.02)$ & $-0.11(-0.16 ;-0.06)$ & $<0.001$ \\
\hline Model 3 & $-0.03(-0.08 ; 0.03)$ & $-0.10(-0.17 ;-0.03)$ & 0.001 \\
\hline Model 4 & $-0.03(-0.08 ; 0.03)$ & $-0.10(-0.17 ;-0.03)$ & 0.004 \\
\hline
\end{tabular}

Regression coefficients $(B)$ indicate the mean difference $(95 \% \mathrm{Cl}$ ) in retinal and skin measures (in \%) with NGM as reference. Standardized regression coefficients (stB) and $95 \% \mathrm{Cl}$ represent the change in retinal and skin measures (in SD) as compared to NGM. SD, standard deviation; $\mathrm{Cl}$, confidence interval.

Model 2a: adjusted for age. Model 2b: as 2a + adjusted for postmenopausal status. Model 2c: as 2a + adjusted for the use of hormone replacement therapy. Model $2 \mathrm{~d}$ : as $2 \mathrm{a}+$ adjusted for postmenopausal status and the use of hormone replacement therapy. Model 3: as $2 d+$ additionally adjusted for body mass index, triglyceride levels, total-to-HDL-cholesterol ratio, smoking status, systolic blood pressure, use of antihypertensive and/or lipid-modifying drugs. Model 4: additionally adjusted for history of cardiovascular disease, retinopathy, eGFR, and urinary albumin excretion. * Menopausal status data were available in $n=1039 / 1083$ women in the retinal reactivity population and in $n=699 / 758$ women in the heat-induced skin hyperemia population. 


\section{Addendum 2.1}

\section{Response to letters regarding article "Prediabetes and type 2 diabetes are associated with generalized microvascular dysfunction: The Maastricht Study"}

Ben M. Sörensen, Alfons J.H.M. Houben, Remy J.H. Martens, Coen D.A. Stehouwer

Circulation. 2017;135:e862-3

We appreciate Dr Tsuda ${ }^{1}$ and Dr Brzezinski ${ }^{2}$ for their interest in our study ${ }^{3}$, in which we found retinal arteriolar and skin microvascular dysfunction in prediabetes and type 2 diabetes (T2D) independent of major cardiovascular risk factors. These findings support the concept that generalized microvascular dysfunction precedes the clinical diagnosis of T2D and may contribute to the development of microvascular complications in (pre)diabetes.

Resting albuminuria has long been favored as clinical biomarker for renal disease and is strongly associated with cardiovascular disease risk $^{4}$. An explanation is that albuminuria may reflect generalized (microvascular and macrovascular) endothelial dysfunction $^{5}$. Data on the association between direct measures of microvascular dysfunction and albuminuria are however scarce. Recently, we have shown an association between capillary rarefaction and 24-h average albuminuria, irrespective of $T 2 D^{5}$. In addition, the results of a yet unpublished study of our group hint towards an association of retinal arteriolar and skin microvascular endothelial dysfunction with 24-h average albuminuria. Both studies support the hypothesis that 24-h average albuminuria is a biomarker of microvascular dysfunction.

The association of exercise-induced albuminuria with features of the metabolic syndrome highlights exercise-induced albuminuria as a possible tool in the early assessment of cardiovascular risk among dysmetabolic individuals ${ }^{6}$. Because of logistical reasons, we did not include urinary albumin excretion measurements after exercise in our study. Whether exercise-induced albuminuria is associated with microvascular dysfunction in (pre)diabetes can therefore not be answered from our data. However, as resting albuminuria may reflect generalized endothelial dysfunction ${ }^{5}$, and prediabetes has been associated with generalized endothelial dysfunction (i.e. in the retinal and skin microvasculature ${ }^{3}$ ) it may be speculated that exercise-induced albuminuria is linked to retinal and skin microvascular endothelial dysfunction in prediabetes, although this inference should be formally tested. 
Taken together, we believe that resting urinary albumin excretion, due to its common use, easy applicability, and its strong association with increased cardiovascular risk, is currently favored over exercise-induced urinary albumin excretion. However, research on earlier markers of renal damage, such as exercise-induced albuminuria, can be useful to improve prevention of renal and cardiovascular disease.

Dr Tsuda suggests that erythrocyte biomechanical properties, which depend on deformability, may be related to retinal and/or skin microvascular dysfunction ${ }^{1}$. Decreased erythrocyte deformability may affect erythrocyte rheology, which could thereby lead to circulatory disorders. Unfortunately, data on erythrocyte deformability are not available, and we therefore cannot directly answer the question based on our own data. Although erythrocyte deformability may conceivably cause microvascular dysfunction, it is important to stress that any such association may also be explained by impaired nitric oxide bioavailability in both endothelial cells and erythrocytes ${ }^{7}$ caused by a common underlying cause, such as inflammatory activity and high levels of free fatty acids $^{8}$. Alternatively, hyperglycemia itself may impair both erythrocyte deformability ${ }^{9}$ and microvascular function.

Decreased deformability of erythrocytes hampers normal passage through capillaries which may lead to mechanical damage of the endothelium, increased platelet activation, and thrombus formation, all of which increase shear stress. Retinal arteriolar \%-dilation, in our study, was measured in relatively large $(>70 \mu \mathrm{m})$ vessels as compared to the diameter of an erythrocyte (6-8 $\mu \mathrm{m})$. Skin microcirculation comprised smaller arterioles and venules than retinal arterioles, with diameters as small as $10 \mu \mathrm{m}$, but probably larger in the heat-induced dilated state. Therefore, whether passage of erythrocytes through retinal and skin microcirculation as measured in our study is truly abnormal remains to be shown.

We agree that future studies should investigate the association between erythrocyte rheological abnormalities and microvascular endothelial dysfunction, which may open new insights to the development of circulatory disorders in (pre)diabetes. 


\section{References}

1. Tsuda K. Letter by Tsuda Regarding Article, "Prediabetes and Type 2 Diabetes Are Associated With Generalized Microvascular Dysfunction: The Maastricht Study". Circulation. 2017;135:e858-9.

2. Brzezinski RY, Berliner S and Shenhar-Tsarfaty S. Letter by Brzezinski et al Regarding Article, "Prediabetes and Type 2 Diabetes Are Associated With Generalized Microvascular Dysfunction: The Maastricht Study". Circulation. 2017;135:e860-1.

3. Sörensen BM, Houben AJ, Berendschot TT, Schouten JS, Kroon AA, van der Kallen CJ, Henry RM, Koster A, Sep SJ, Dagnelie PC, Schaper NC, Schram MT and Stehouwer CD. Prediabetes and Type 2 Diabetes Are Associated With Generalized Microvascular Dysfunction: The Maastricht Study. Circulation. 2016;134: 1339-52.

4. Chronic Kidney Disease Prognosis C, Matsushita K, van der Velde M, Astor BC, Woodward M, Levey AS, de Jong PE, Coresh J and Gansevoort RT. Association of estimated glomerular filtration rate and albuminuria with all-cause and cardiovascular mortality in general population cohorts: a collaborative meta-analysis. Lancet. 2010;375:2073-81.

5. Martens RJ, Henry RM, Houben AJ, van der Kallen CJ, Kroon AA, Schalkwijk CG, Schram MT, Sep SJ, Schaper NC, Dagnelie PC, Muris DM, Gronenschild EH, van der Sande FM, Leunissen KM, Kooman JP and Stehouwer CD. Capillary Rarefaction Associates with Albuminuria: The Maastricht Study. J Am Soc Nephrol. 2016;27:3748-57.

6. Greenberg S, Shenhar-Tsarfaty S, Rogowski O, Shapira I, Zeltser D, Weinstein T, Lahav D, Vered J, ToviaBrodie $O$, Arbel $Y$, Berliner $S$ and Milwidsky A. Exercise-induced albuminuria is related to metabolic syndrome. Am J Physiol Renal Physiol. 2016;310:F1192-6.

7. Grau M, Pauly S, Ali J, Walpurgis K, Thevis M, Bloch W and Suhr F. RBC-NOS-dependent S-nitrosylation of cytoskeletal proteins improves RBC deformability. PLoS One. 2013;8:e56759.

8. Muris DM, Houben AJ, Schram MT and Stehouwer CD. Microvascular dysfunction: an emerging pathway in the pathogenesis of obesity-related insulin resistance. Rev Endocr Metab Disord. 2013;14:29-38.

9. Keymel S, Heiss C, Kleinbongard P, Kelm M and Lauer T. Impaired red blood cell deformability in patients with coronary artery disease and diabetes mellitus. Horm Metab Res. 2011;43:760-5. 



\section{Chapter}

Hyperglycemia is the main mediator of prediabetes-

and type 2 diabetes-associated impairment of microvascular function: The Maastricht Study

Ben M. Sörensen, Alfons J.H.M. Houben, Tos T.J.M. Berendschot, Jan S.A.G. Schouten, Abraham A. Kroon, Carla J.H. van der Kallen, Ronald M.A. Henry, Annemarie Koster, Koen D. Reesink, Pieter C. Dagnelie, Nicolaas C. Schaper, Casper G. Schalkwijk, Miranda T. Schram, Coen D.A. Stehouwer Observation letter: Diabetes Care. 2017;40:e103-5 


\section{Abstract}

\section{Objective}

In individuals with prediabetes and type 2 diabetes (T2D), microvascular dysfunction may explain their increased risk of microvascular complications. Mechanisms underlying microvascular dysfunction in (pre)diabetes remain poorly understood. We investigated to what extent differences in retinal and skin microvascular function between individuals without and with (pre)diabetes are potentially attributable to hyperglycemia, insulin resistance, blood pressure, lipid profile, and/or low-grade inflammation.

\section{Methods}

In The Maastricht Study, a T2D-enriched population-based cohort study ( $n=1791,51 \%$ men, aged $60 \pm 8$ years), we determined flicker light-induced retinal arteriolar \%-dilation (Dynamic Vessel Analyzer), heat-induced skin \%-hyperemia (laser-Doppler flowmetry), and diabetes status (OGTT; normal glucose metabolism (NGM) $(n=1040)$, prediabetes $(n=276)$, or T2D $(n=475))$. Composite indices were formed of hyperglycemia, insulin resistance, blood pressure, lipid profile, and lowgrade inflammation. Mediating effects of composite indices on prediabetes- and T2D-associated microvascular dysfunction were estimated by linear regression.

\section{Results}

Age- and sex-adjusted analyses showed lower retinal arteriolar \%-dilation in prediabetes $(B=-0.16,95 \% \mathrm{Cl}(-0.53 ; 0.21))$, with further deterioration in T2D $(B=-0.83,(-1.15 ;-0.51))$ versus NGM, $P$ for trend $<0.001$. Skin \%-hyperemia was lower in prediabetes $(B=-80,(-198 ; 38))$, with further deterioration in T2D ( $B=-210,(-309 ;-112))$ versus NGM, $P$ for trend<0.001. T2D-associated differences in microvascular function were explained mainly by hyperglycemia (mediating effect (bootstrapped $95 \% \mathrm{Cl}) 55.3 \%(20.4 \% ; 91.3 \%)$ and 64.8\% (6.2\%; 122.4\%), respectively). Other composite indices did not significantly contribute. Patterns of mediation were similar for prediabetes-associated microvascular dysfunction.

\section{Conclusions}

Our findings suggest early and intensive glycemic control in (pre)diabetes as a promising therapeutic target for the prevention of (pre)diabetes-associated microvascular complications. 


\section{Introduction}

The epidemic of type 2 diabetes (T2D) ${ }^{1}$ also implies an epidemic of its macrovascular ${ }^{1,2}$ and microvascular complications ${ }^{3}$. The latter not only comprises retinopathy and nephropathy ${ }^{2}$, but also complications that are partly of microvascular origin, notably neuropathy, heart failure ${ }^{4}$, stroke, depression ${ }^{5}$, and cognitive dysfunction ${ }^{6}$. The increasing life expectancy of individuals with $\mathrm{T}^{2} \mathrm{D}^{1}$ highlights the need to develop preventive interventions for the disease itself and its complications.

Recently, we have shown that T2D, as well as prediabetes, is associated with generalized microvascular (endothelial) dysfunction ${ }^{7}$. These findings support the ticking clock hypothesis ${ }^{8}$, and may explain the increased risk of microvascular complications in individuals with prediabetes and early diabetes. However, the mechanisms underlying microvascular dysfunction in (pre)diabetes remain elusive. Multiple studies suggest that both metabolic and/or vascular risk factors are likely to be involved ${ }^{9-13}$.

Key metabolic features of T2D are progressive insulin resistance and $\beta$-cell dysfunction, leading to hyperglycemia and abnormal insulin signaling. Both may impair microvascular function, for instance via the formation of advanced glycation end products, reactive oxygen species, and impaired nitric oxide signaling ${ }^{12,14-16}$. In addition, T2D is strongly associated with an adverse cardiovascular risk profile, with low-grade inflammation $^{17}$, dyslipidemia ${ }^{18}$, hypertension ${ }^{19}$, and arterial stiffness ${ }^{20}$. Importantly, there is increasing evidence that each of these comorbid vascular risk factors is also linked to microvascular dysfunction and may therefore explain concordant microvascular complications, for instance, through adverse effects on the insulin signaling pathway, impairment of nitric oxide bioavailability, abnormal regulation of vasomotor tone and vascular rarefaction, and/or increased pulsatile flow ${ }^{9,11,21-23 .}$

However, it remains unclear to what extent such metabolic and vascular risk factors also mediate, and thus explain, diabetes-associated microvascular dysfunction. Therefore, the present study aimed to investigate to what extent differences in retinal and skin microvascular function between individuals with $\mathrm{T} 2 \mathrm{D}$ or prediabetes on the on hand, and those with normal glucose metabolism (NGM) on the other, are attributable to hyperglycemia, insulin resistance, blood pressure, arterial stiffness, lipid profile, and low-grade inflammation, independently of potential confounders. We chose the retina and skin as these are unique sites enabling direct and reproducible ${ }^{24,25}$ assessment of microvascular function, as measured by flicker light-induced retinal arteriolar dilation and heat-induced skin hyperemia ${ }^{7}$. 


\section{Methods}

\section{Study population and design}

We used data from The Maastricht Study, an observational prospective populationbased cohort study. The rationale and methodology are outlined in the Supplemental Methods and have been described previously ${ }^{26}$. The present report includes crosssectional data from the first 3451 participants, who completed the baseline survey between November 2010 and September 2013. The study has been approved by the institutional medical ethical committee (NL31329.068.10) and the Minister of Health, Welfare and Sports of the Netherlands (Permit 131088-105234-PG). All participants gave written informed consent.

\section{Assessment of glucose metabolism status}

To assess glucose metabolism status, all participants (except those who used insulin) underwent a standardized 2-h 75 gram oral glucose tolerance test (OGTT) as described elsewhere ${ }^{26}$ and in the Supplemental Methods.

\section{Assessment of microvascular function}

Retinal arteriolar vasodilation to flicker light exposure was measured by the Dynamic Vessel Analyzer (Imedos, Jena, Germany), and heat-induced skin hyperemia by laserDoppler flowmetry (Perimed, Järfälla, Sweden), both extensively described previously ${ }^{7}$ and in the Supplemental Methods.

\section{Measurement of potential mediators}

For statistical efficiency, a composite index of hyperglycemia was constructed, which consisted of fasting (FPG) and 2-h postload OGTT glucose levels, representing short-term hyperglycemia, and glycated hemoglobin $\mathrm{A} 1 \mathrm{c}(\mathrm{HbA} 1 \mathrm{c})$ and skin auto-fluorescence (SAF), representing long-term hyperglycemia. The individual measures were standardised into z-scores and averaged. The z-score for each individual measure was calculated according to the formula: (individual value / population mean) / population standard deviation. Individuals who used insulin had no data on 2-h postload glucose as they were excluded from the OGTT; hence, their composite index of hyperglycemia consisted of FPG, HbA1c, and SAF. For sensitivity purposes, a composite index that focused on long-term hyperglycemic measures was formed, as FPG and postload glucose levels were directly used for the classification of glucose metabolism status. 
The composite index of insulin resistance consisted of 2 homeostasis model

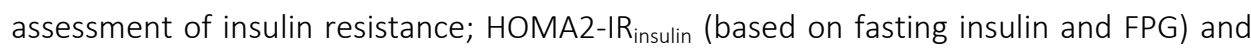
HOMA2-IR $\mathrm{R}_{\mathrm{C} \text {-peptide }}$ (based on fasting C-peptide and FPG).

The composite index of blood pressure consisted of 24-h ambulatory systolic and diastolic blood pressure combined with the use of antihypertensive medication in order to account for potential previous exposure to elevated blood pressure. For sensitivity analyses, an index that combined 24-h mean arterial pressure and 24-h pulse pressure with the use of antihypertensive medication was constructed, as elevated pulse pressure may adversely affect the microcirculation ${ }^{9}$.

The composite index of arterial stiffness consisted of the carotid-femoral pulse wave velocity (as marker for aortic stiffness) and the inverse of the carotid distensibility coefficient (as marker for local carotid stiffness).

The composite index of lipid profile consisted of total cholesterol, inverse HDL-cholesterol, triglyceride levels, and the use of lipid-modifying medication in order to account for potential previous exposure to an adverse lipid profile.

The low-grade inflammation composite index consisted of high-sensitive C-reactive protein (hs-CRP), serum amyloid A (SAA), soluble intercellular adhesion molecule-1, interleukin-6, interleukin-8, and tumor necrosis factor- $\alpha$ (TNF- $\alpha$ ). This index was formed to reduce the influences of the biological variability of each inflammation marker, according to predefined clusters of conceptually related circulating biomarkers ${ }^{27}$. Details on the measurement of potential mediators can be found in the Supplemental Methods.

\section{Measurement of covariates}

Details on the assessments of the covariates, age, sex, body mass index, smoking status, estimated glomerular filtration rate (eGFR), urinary albumin excretion, the presence of retinopathy, and history of cardiovascular disease, can be found in the Supplemental Methods.

\section{Statistical analysis}

All analyses were performed with Statistical Package for Social Sciences version 23.0 (IBM SPSS, Armonk, USA) at a significance level of 5\%. Variables with a skewed distribution (diabetes duration, hs-CRP, SAA, interleukin-6, interleukin-8, and TNF- $\alpha$ ) were log10 transformed.

Multiple linear regression analysis, with dummy variables for prediabetes and T2D, was used to determine the age- and sex-adjusted difference in flicker light-induced retinal arteriolar \%-dilation or heat-induced skin \%-hyperemia between individuals with prediabetes or T2D, versus NGM. These adjusted differences constitute the total 'effect' 
of prediabetes or T2D on retinal arteriolar \%-dilation or heat-induced skin \%-hyperemia (Supplemental Figure S3.1). For linear trend analyses, a categorical variable (NGM=0, prediabetes $=1$, and $T 2 \mathrm{D}=2$ ) was used in the regression models.

The mediating effects of the individual markers of hyperglycemia, insulin resistance, blood pressure, lipid profile, and low-grade inflammation on diabetes-associated retinal or skin microvascular function were evaluated by bootstrapping, by adding these variables separately to the age- and sex-adjusted regression model. The bootstrappedadjusted mediating effects and corresponding 95\% confidence intervals $(95 \% \mathrm{Cl})$ were evaluated with use of a SPSS macro provided by Preacher and Hayes (10,000 bootstrap iterations) $^{28}$, and expressed as the (percentage) change of the regression coefficients (indirect effect) (Supplemental Figure S3.1). The indirect effect is the product of the association between T2D and the potential mediator (a-path) and the association between the potential mediator and microvascular function ( $b$-path). Indirect effects in which the $95 \% \mathrm{Cl}$ does not include zero reflect a significant mediation effect. Estimation of the indirect effect with bootstrapping does not depend on the significance of the separate $a$ - and $b$-paths, and therefore maintains reasonable control over the type 1 error rate ${ }^{28}$ (Supplemental Figure S3.1). Selection of potential mediators on theoretical grounds rather than on a statistical basis has therefore been recommended and was applied here ${ }^{28}$.

To justify the merging of individual markers within a composite index, the individual mediating effect of the markers of that index should have the same direction or be zero (defined as a mediation effect of less than $1 \%$ of the average retinal arteriolar \%-dilation or skin \%-hyperemia in the respective study populations). The extent to which the composite indices mediate the difference in retinal or skin microvascular function between individuals with $\mathrm{T} 2 \mathrm{D}$ or prediabetes, versus individuals with NGM, was determined by adding these composites indices combined to the age- and sex-adjusted regression model.

We investigated potential interaction between sex and glucose metabolism status by adding interaction terms (prediabetes $*$ sex and $\mathrm{T} 2 \mathrm{D} * \mathrm{sex}$ ) to the regression models. $A \mathrm{P}_{\text {interaction }}<0.10$ was considered statistically significant. Collinearity diagnostics (i.e. tolerance $<0.1$ and/or variance inflation factor $>10$ ) were used to detect multicollinearity between the independent variables and mediators. 


\section{Results}

\section{Study population}

From the initial 3451 participants included, participants with other types of diabetes than T2D were excluded ( $n=41)$. Of the remaining 3410 participants, retinal arteriolar reactivity data were available in 2261 participants. The main reasons for missing data were logistical $(n=882)$, contraindications $(n=59)$, or insufficient measurement quality $(n=208)$. Data on potential mediators, in particular 24-h ambulatory blood pressure $(n=256)$, were missing in 470 participants, mainly due to device availability. The retinal arteriolar reactivity study population thus consisted of 1791 participants. Heat-induced skin hyperemia data were available in 1647 of the 3410 participants. The reason for missing data were logistical $(n=1650)$, technical $(n=24)$, or insufficient measurement quality $(n=89)$. Potential mediators were missing in 366 participants. The heat-induced skin hyperemia study population thus consisted of 1281 participants (Figure 3.1 shows the flow chart).

General characteristics of the retinal study population are shown in Table 3.1, stratified for glucose metabolism status (mean \pm standard deviation age $59.8 \pm 8.2$ years, $48.7 \%$ women). Individuals with T2D were, by design, oversampled (26.5\%). The cardiometabolic risk profile deteriorated with glucose metabolism status (Table 3.1). The skin study population overlapped for $73 \%$ with the retinal study population, and was comparable with regard to age, sex, and cardiometabolic risk profile (Table 3.1 and Supplemental Table S3.1). Note that individuals with diabetes were generally well controlled for their diabetes and cardiovascular risk factors, as, for example, absolute differences in 24-h blood pressure between individuals with diabetes and those with NGM were relatively small (122/73 versus $117 / 74 \mathrm{mmHg}$ ) as were the absolute differences in total-to-HDL cholesterol ratio $(3.7$ versus $3.5 \mathrm{mmol} / \mathrm{l})$. Individuals with missing data on retinal or skin reactivity measurements or potential mediators were generally comparable to individuals included in the study populations with regard to age, sex, and cardiometabolic risk profile (Supplemental Tables S3.2 and S3.3). 


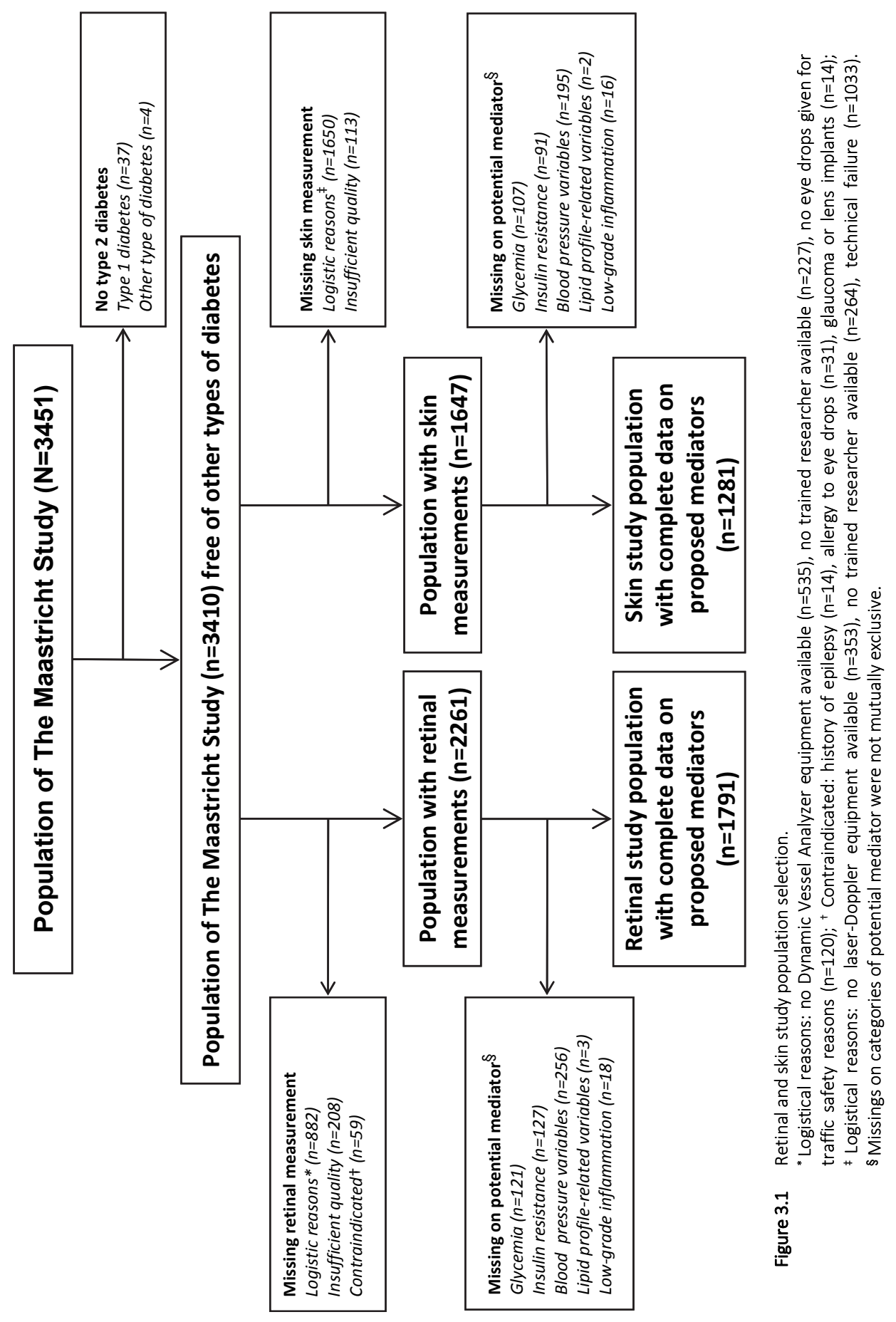


Table 3.1 General characteristics and retinal and skin measures of the retinal study population according to glucose metabolism status

\begin{tabular}{|c|c|c|c|}
\hline Characteristic & $\begin{array}{c}\text { NGM } \\
n=1040\end{array}$ & $\begin{array}{c}\text { Prediabetes } \\
n=276\end{array}$ & $\begin{array}{c}\text { Type } 2 \text { diabetes } \\
\mathrm{n}=475\end{array}$ \\
\hline Age (years) & $58.0 \pm 8.2$ & $61.5 \pm 7.2$ & $62.9 \pm 7.6$ \\
\hline Women & $596(57.3)$ & $130(47.1)$ & $147(30.9)$ \\
\hline Diabetes duration (years) ${ }^{*}$ & - & - & $6.0[3.0-12.0]$ \\
\hline Body mass index $\left(\mathrm{kg} / \mathrm{m}^{2}\right)$ & $25.5 \pm 3.4$ & $27.5 \pm 4.0$ & $29.5 \pm 4.5$ \\
\hline \multicolumn{4}{|l|}{ Waist circumference $(\mathrm{cm})$} \\
\hline - Men & $96.3 \pm 9.1$ & $101.6 \pm 9.4$ & $106.7 \pm 11.6$ \\
\hline - Women & $85.6 \pm 9.7$ & $92.7 \pm 12.1$ & $100.9 \pm 13.6$ \\
\hline History of cardiovascular disease & $116(11.4)$ & $30(11.2)$ & $118(25.8)$ \\
\hline Office SBP (mmHg) & $131.3 \pm 17.3$ & $137.1 \pm 16.7$ & $141.6 \pm 17.5$ \\
\hline Office DBP (mmHg) & $75.5 \pm 10.0$ & $78.2 \pm 9.7$ & $77.4 \pm 9.8$ \\
\hline Ambulatory 24-h SBP (mmHg) & $117.3 \pm 11.1$ & $120.1 \pm 11.9$ & $122.4 \pm 11.7$ \\
\hline Ambulatory 24-h DBP (mmHg) & $73.6 \pm 7.2$ & $74.5 \pm 7.3$ & $73.0 \pm 7.0$ \\
\hline Ambulatory 24-h PP (mmHg) & $43.7 \pm 7.1$ & $45.6 \pm 8.7$ & $49.5 \pm 9.6$ \\
\hline Ambulatory 24-h MAP (mmHg) & $88.2 \pm 8.1$ & $89.7 \pm 8.1$ & $89.5 \pm 7.7$ \\
\hline \multicolumn{4}{|l|}{ Smoking } \\
\hline - Never / former / current & $409 / 502 / 116$ & $75 / 161 / 33$ & $136 / 264 / 62$ \\
\hline - \% (never / former / current) & $39.8 / 48.9 / 11.3$ & 27.9/59.9/12.3 & 29.4/57.1/13.4 \\
\hline Fasting glucose (mmol/l) & $5.2 \pm 0.4$ & $5.9 \pm 0.6$ & $7.7 \pm 1.7$ \\
\hline 2-h postload glucose $(\mathrm{mmol} / \mathrm{l})^{+}$ & $5.4 \pm 1.1$ & $8.2 \pm 1.7$ & $14.2 \pm 3.9$ \\
\hline $\mathrm{HbA1c}(\%)$ & $5.4 \pm 0.4$ & $5.7 \pm 0.4$ & $6.8 \pm 0.9$ \\
\hline $\mathrm{HbA} 1 \mathrm{c}(\mathrm{mmol} / \mathrm{mol})$ & $35.8 \pm 3.7$ & $38.4 \pm 4.5$ & $50.6 \pm 9.9$ \\
\hline Skin auto-fluorescence (AU) & $2.3 \pm 0.5$ & $2.4 \pm 0.5$ & $2.6 \pm 0.6$ \\
\hline Total-to-HDL cholesterol ratio & $3.5 \pm 1.1$ & $3.9 \pm 1.3$ & $3.7 \pm 1.1$ \\
\hline Total cholesterol (mmol/l) & $5.6 \pm 1.0$ & $5.5 \pm 1.2$ & $4.4 \pm 1.1$ \\
\hline HDL cholesterol (mmol/l) & $1.7 \pm 0.5$ & $1.5 \pm 0.4$ & $1.3 \pm 0.4$ \\
\hline LDL cholesterol (mmol/I) & $3.3 \pm 0.9$ & $3.3 \pm 1.1$ & $2.4 \pm 0.9$ \\
\hline Triglycerides (mmol/l) & $1.2 \pm 0.6$ & $1.6 \pm 1.0$ & $1.8 \pm 0.9$ \\
\hline Antihypertensive medication use & $226(21.7)$ & $113(40.9)$ & $333(70.1)$ \\
\hline Lipid-modifying medication use & $178(17.1)$ & $93(33.7)$ & $350(73.7)$ \\
\hline \multicolumn{4}{|l|}{ Diabetes medication use } \\
\hline - Any type & - & - & $359(75.6)$ \\
\hline - Insulin & - & - & $86(18.1)$ \\
\hline - Oral glucose-lowering medication & - & - & $338(71.2)$ \\
\hline HOMA2-IR Insulin $(A U)$ & $1.3 \pm 0.7$ & $1.9 \pm 1.1$ & $2.4 \pm 1.4$ \\
\hline HOMA2-IR c-peptide $(A U)$ & $1.3 \pm 0.5$ & $1.7 \pm 0.7$ & $2.1 \pm 0.9$ \\
\hline \multicolumn{4}{|l|}{ Markers of low-grade inflammation } \\
\hline - High-sensitive CRP (mg/l) & $1.1[0.6-2.2]$ & $1.8[0.8-3.5]$ & $1.5[0.7-3.3]$ \\
\hline - Serum amyloid A (mg/l) & $3.0[1.9-5.0]$ & $3.6[2.3-5.7]$ & $3.5[2.2-6.0]$ \\
\hline - Soluble ICAM-1 (ng/ml) & $338.6 \pm 80.2$ & $365.8 \pm 103.2$ & $383.8 \pm 115.9$ \\
\hline - Interleukin-6 (pg/ml) & $0.5[0.4-0.8]$ & $0.6[0.4-0.9]$ & $0.8[0.6-1.1]$ \\
\hline - Interleukin-8 (pg/ml) & $3.7[3.0-4.6]$ & $4.3[3.3-5.3]$ & $4.8[4.0-6.1]$ \\
\hline - Tumor necrosis factor- $\alpha$ (pg/ml) & $2.1[1.8-2.4]$ & $2.2[1.9-2.6]$ & $2.5[2.1-2.9]$ \\
\hline \multicolumn{4}{|l|}{ Markers of arterial stiffness } \\
\hline - Carotid-femoral PWV (m/s) & $8.4 \pm 1.7$ & $9.2 \pm 2.1$ & $9.9 \pm 2.3$ \\
\hline - Carotid DC $\left(10^{3} / \mathrm{kPa}\right)$ & $15.1 \pm 5.2$ & $13.7 \pm 4.8$ & $13.3 \pm 4.9$ \\
\hline eGFR $\left(\mathrm{ml} / \mathrm{min} / 1.73 \mathrm{~m}^{2}\right)$ & $89.9 \pm 13.0$ & $87.2 \pm 14.0$ & $85.3 \pm 17.2$ \\
\hline $\mathrm{eGFR}<60 \mathrm{ml} / \mathrm{min} / 1.73 \mathrm{~m}^{2}$ & $18(1.7)$ & $10(3.6)$ & $45(9.5)$ \\
\hline
\end{tabular}


Table $3.1 \quad$ (continued)

\begin{tabular}{|c|c|c|c|}
\hline Characteristic & $\begin{array}{c}\text { NGM } \\
n=1040\end{array}$ & $\begin{array}{c}\text { Prediabetes } \\
n=276\end{array}$ & $\begin{array}{c}\text { Type } 2 \text { diabetes } \\
n=475\end{array}$ \\
\hline (Micro)albuminuria $^{\ddagger}$ & $47(4.6)$ & $15(5.4)$ & $77(16.2)$ \\
\hline Retinopathy & $1(0.1)$ & $1(0.4)$ & $18(3.9)$ \\
\hline Baseline arteriolar diameter (MU) & $115.3 \pm 15.3$ & $114.8 \pm 15.9$ & $116.0 \pm 15.9$ \\
\hline \multicolumn{4}{|l|}{ Arteriolar average dilation (\%) } \\
\hline - Mean \pm SD & $3.4 \pm 2.8$ & $3.1 \pm 2.8$ & $2.4 \pm 2.7$ \\
\hline - Median [interquartile range] & $3.0[1.1-5.3]$ & $2.8[0.8-5.0]$ & $1.6[0.4-3.9]$ \\
\hline Baseline skin blood flow $(\mathrm{PU})^{\S}$ & $10.8 \pm 6.4$ & $11.7 \pm 7.2$ & $11.0 \pm 5.7$ \\
\hline \multicolumn{4}{|l|}{ Skin hyperemic response $(\%)^{\S}$} \\
\hline- Mean $\pm S D$ & $1252.6 \pm 813.4$ & $1107.4 \pm 710.8$ & $941.7 \pm 701.1$ \\
\hline - Median [interquartile range] & $1104.0[668.7-1656.9]$ & $1006.9[604.9-1536.9]$ & 821.2 [479.0-1209.8] \\
\hline
\end{tabular}

Data are reported as mean $\pm \mathrm{SD}$, median [interquartile range], or number (percentage \%) as appropriate. NGM, normal glucose metabolism; SD, standard deviation; SBP, systolic blood pressure; DBP, diastolic blood pressure; PP, pulse pressure; MAP, mean arterial pressure; HbA1c, glycated hemoglobin A1c; AU, arbitrary units; HDL, high-density lipoprotein; LDL, low-density lipoprotein; HOMA2-IR, homeostasis model assessment of insulin resistance; CRP, C-reactive protein; ICAM, intercellular adhesion molecule; PWV, pulse wave velocity; DC, distensibility coefficient; eGFR, estimated glomerular filtration rate; MU, measurement units; PU, perfusion units. ${ }^{*}$ Available in 313 individuals with type 2 diabetes. ${ }^{+}$Available in 389 individuals with type 2 diabetes. ${ }^{\ddagger}$ (Micro)albuminuria was defined as a urinary albumin excretion of $>30 \mathrm{mg}$ per 24 hours. ${ }^{\S}$ Heat-induced skin hyperemia measures were available in a different subset of $n=1281$ (Supplemental Table S3.1).

\section{Glucose metabolism status and retinal and skin microvascular function}

Age- and sex-adjusted analyses showed a lower retinal arteriolar \%-dilation in prediabetes $(B=-0.16,95 \% \mathrm{Cl}(-0.53 ; 0.21))$, with further deterioration in $T 2 D(B=-0.83$, $(-1.15 ;-0.51))$ versus NGM, $P$ for trend<0.001. Skin \%-hyperemia was also lower in prediabetes $(B=-80,(-198 ; 38))$, with further deterioration in T2D $(B=-210,(-309 ;-112))$ versus NGM, $P$ for trend $<0.001$. These regression coefficients slightly differ from earlier work $^{7}$, as a smaller subset of individuals from The Maastricht Study was available (Figure 3.1). Despite the overall trend towards lower retinal arteriolar \%-dilation and skin \%-hyperemia with deteriorating glucose metabolism status, statistically significant impaired retinal and skin microvascular function was observed only in individuals with T2D. In addition, the association of prediabetes and T2D with retinal arteriolar \%-dilation and skin \%-hyperemia did not statistically differ between men and women (all $P_{\text {interaction }}>0.15$ ).

\section{Mediating effects of markers of hyperglycemia, insulin resistance, blood pressure, lipid profile, and low-grade inflammation on type 2 diabetes- associated retinal and skin microvascular dysfunction}

Figure 3.2 and Supplemental Table S3.4 show the age- and sex-adjusted mediating effects of individual markers of hyperglycemia, insulin resistance, blood pressure, lipid 
profile, and low-grade inflammation on the differences in retinal arteriolar \%-dilation and skin \%-hyperemia between individuals with T2D and those with NGM. Diabetesassociated impaired retinal arteriolar \%-dilation was mostly explained by markers of hyperglycemia, with $\mathrm{HbA} 1 \mathrm{c}(49.3 \%$ bootstrapped $95 \% \mathrm{Cl}(14.5 ; 80.2 \%))$ and FPG $(50.3 \%$ $(13.5 ; 87.3 \%))$ as the most prominent mediators. The diabetes-associated difference in retinal arteriolar \%-dilation was also mediated, although to a lesser extent, by 24-h diastolic blood pressure $(2.8 \%(0.3 ; 7.4 \%))$, the use of antihypertensive medication $(15.3 \%(0.6 ; 31.1 \%))$ and interleukin-6 $(7.5 \%(0.6 ; 14.9 \%))$. The diabetes-associated difference in skin \%-hyperemia was, besides HbA1c (46.2\% (9.3; 84.3\%)), not significantly mediated by any of the other individual markers which constitute the composite indices.
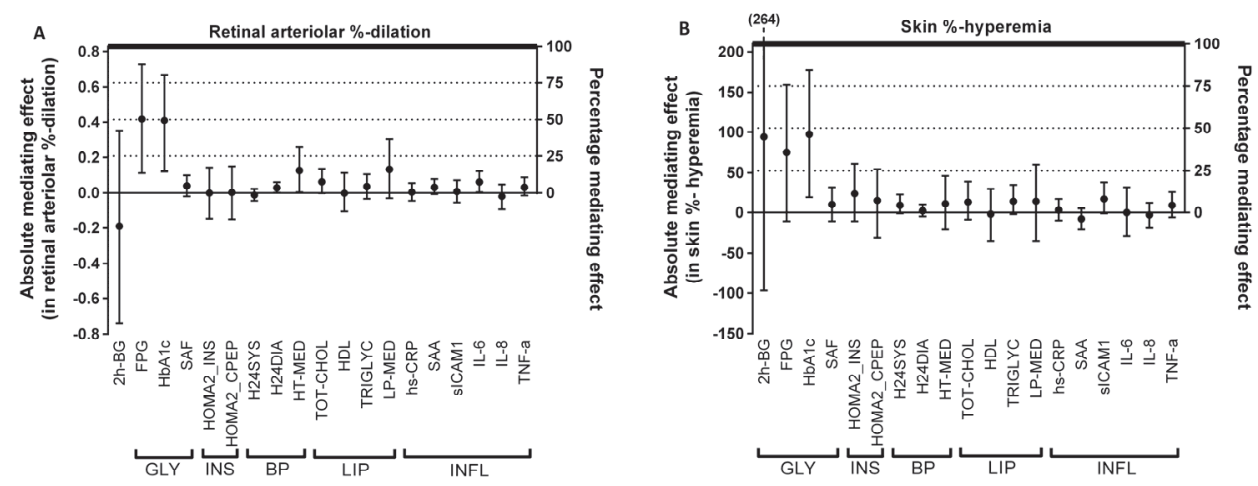

Figure 3.2 Age- and sex-adjusted mediating effects of markers of hyperglycemia, insulin resistance, blood pressure, lipid profile, and low-grade inflammation on the difference in retinal arteriolar \%-dilation (A) and skin \%-hyperemia (B) between individuals with type 2 diabetes and normal glucose metabolism.

Mediating effects are presented as the indirect effects of type 2 diabetes on retinal arteriolar \%-dilation (left) and skin \%-hyperemia (right) through the potential mediators in \%-microvascular function [left Y-axis] and percentage of mediation [right Y-axis]. All potential mediators are adjusted for age and sex. The thick horizontal line indicates the mean difference in retinal arteriolar \%-dilation (left) and skin \%-hyperemia (right) between individuals with type 2 diabetes and those with normal glucose metabolism, and thus corresponds with a mediation effect of $100 \%$. Mediating effects of markers of blood pressure and lipid profile are additionally adjusted for the use of antihypertensive or lipid-modifying medication, respectively. FPG, fasting glucose; 2h-BG, 2-h postload glucose; HbA1c, glycated hemoglobin A1c; SAF, skin auto-fluorescence; HOMA2_INS, homeostasis model assessment of insulin resistance based on fasting plasma insulin and FPG; HOMA2_CPEP, homeostasis model assessment of insulin resistance based on fasting C-peptide and FPG; H24SYS, 24-h systolic blood pressure; H24DIA, 24-h diastolic blood pressure; HT-med, use of antihypertensive medication; Tot-chol, total cholesterol; HDL, inverse high-density lipoprotein cholesterol; triglyc, triglycerides; LP-med, use of lipid-modifying medication; hs-CRP, high-sensitive C-reactive protein; SAA, serum amyloid A; sICAM1, soluble intercellular adhesion molecule-1; IL-6, interleukin-6; IL-8, interleukin-8; and TNF-a, tumor necrosis factor $-\alpha$. GLY = composite index of FPG, $\mathrm{HbA1c}$, and SAF for retinal arteriolar reactivity analyses, and composite index of FPG, 2-h postload glucose, HbA1c, and SAF for skin reactivity analyses; INS = composite index of 2 homeostasis model assessment of insulin resistance, 
1 based on fasting plasma insulin and FPG, and the other based on fasting C-peptide and FPG; $\mathrm{BP}=$ composite index that combined 24-h systolic and diastolic blood pressure with the use of antihypertensive medication; LIP = composite index that combined total cholesterol, inverse high-density lipoprotein cholesterol, and triglycerides with the use of lipid-modifying medication; INFL = composite index that combined high-sensitive C-reactive protein, serum amyloid A, soluble intercellular adhesion molecule-1, interleukin-6, interleukin-8, and tumor necrosis factor- $\alpha$.

\section{Mediating effects of composite indices for hyperglycemia, insulin resistance, blood pressure, lipid profile, and low-grade inflammation on type 2 diabetes-associated retinal and skin microvascular dysfunction}

For analyses on retinal arteriolar reactivity, 2-h postload glucose was not incorporated in the hyperglycemia composite index (Figure 3.2 and Supplemental Table S3.4), as the mediating effect of 2-h postload on the T2D-associated difference in retinal arteriolar \%-dilation was directed opposite to those of FPG, HbA1c, and SAF. All other composite indices could justifiably be formed. The hyperglycemia index mainly explained the age- and sex-adjusted difference in retinal arteriolar \%-dilation and skin \%-hyperemia between individuals with T2D and those with NGM with statistically significant mediating effects of $55.3 \%(20.4 ; 91.3 \%)$ and $64.8 \%$ (6.2; 122.4\%), respectively (Figure 3.3A and $3.3 \mathrm{~B})$. In contrast, insulin resistance, blood pressure, lipid profile, and low-grade inflammation indices were no significant mediators of this difference (Figure 3.3A and 3.3B). A similar pattern of mediation was observed for the prediabetes-associated difference in retinal arteriolar \%-dilation or skin \%-hyperemia (Figure 3.3C and 3.3D).

Additional adjustment for smoking status and body mass index as covariates did not materially change these results, except that the mediating effect of the hyperglycemia index was somewhat smaller and not statistically significant for skin \%-hyperemia (Supplemental Figure S3.2) (for retinal and skin analyses, data on covariates were available in $n=1756$ and $n=1239$ individuals, respectively). Similar results were found when body mass index was substituted by waist circumference (data not shown). Moreover, none of the additional covariates had a significant mediation effect on the difference in retinal arteriolar \%-dilation or skin \%-hyperemia between individuals with T2D and NGM (data not shown). Additional adjustment for history of cardiovascular disease, retinopathy, eGFR, and urinary albumin excretion, again did not materially change these results (Supplemental Figure S3.3) (for retinal and skin analyses, data on these additional covariates were available in $n=1676$ and $n=1103$ individuals, respectively). 


\section{Additional analyses}

Qualitatively similar mediating effects of the composite indices of potential mediators on the difference in retinal arteriolar \%-dilation or skin \%-hyperemia between individuals with T2D and NGM were observed in a range of additional analyses. First, when for retinal arteriolar reactivity analyses, 2-h postload glucose (with an opposed individual mediation effect as compared to FPG, HbA1c, and SAF) was added to the composite index of hyperglycemia (data not shown). Second, when we used absolute retinal arteriolar diameter corrected for baseline arteriolar diameter, or absolute skin blood flow corrected for baseline skin blow flow as outcomes, rather than their percentages (data not shown). Third, when a composite index of arterial stiffness was added as potential mediator (for retinal and skin analyses, data on arterial stiffness were available in $n=1453$ and $n=1090$ individuals, respectively) (Supplemental Figure S3.4). Moreover, the composite index of arterial stiffness did not significantly mediate the diabetesassociated impairment in retinal or skin microvascular function. Fourth, when the composite index of hyperglycemia consisted only of long-term hyperglycemic measures (HbA1c and SAF), its mediating effect and corresponding 95\% confidence interval $(95 \% \mathrm{Cl})$ became somewhat smaller (and non-significant for skin \%-hyperemia; Supplemental Figure S3.5). Fifth, when the mediating effect of the blood pressure index was re-analyzed with a composite index that combined the use of antihypertensive medication with 24-h mean arterial pressure and 24-h pulse pressure, rather than 24-h systolic and diastolic blood pressure (Supplemental Figure S3.6). Sixth, when the individual mediating effect of antihypertensive medication was further specified into renin-angiotensin-aldosterone system (RAAS)-inhibiting (with or without other types of antihypertensives) and non-RAAS-inhibiting antihypertensives only (data not shown). RAAS-inhibiting antihypertensives included angiotensin-converting-enzyme inhibitors, angiotensin receptor blockers, and renin blockers. Seventh, when the composite index of low-grade inflammation only included individuals with hs-CRP $<10 \mathrm{mg} /$ l, (for retinal and skin analyses, $n=69$ and $n=58$ individuals were excluded) (Supplemental Figure S3.7). Last, a similar pattern of mediation was found when composite indices were formed when participants had data on at least one of the individual markers which constitute these indices (for retinal and skin analyses, data were then available in $n=2237$ and $\mathrm{n}=1630$ individuals, respectively) (Supplemental Figure S3.8). Collinearity diagnostics revealed no problematic multicollinearity in any of the mediation analyses (i.e. all tolerance values $\geq 0.1$ and variance inflation factors $\leq 10$ ). 

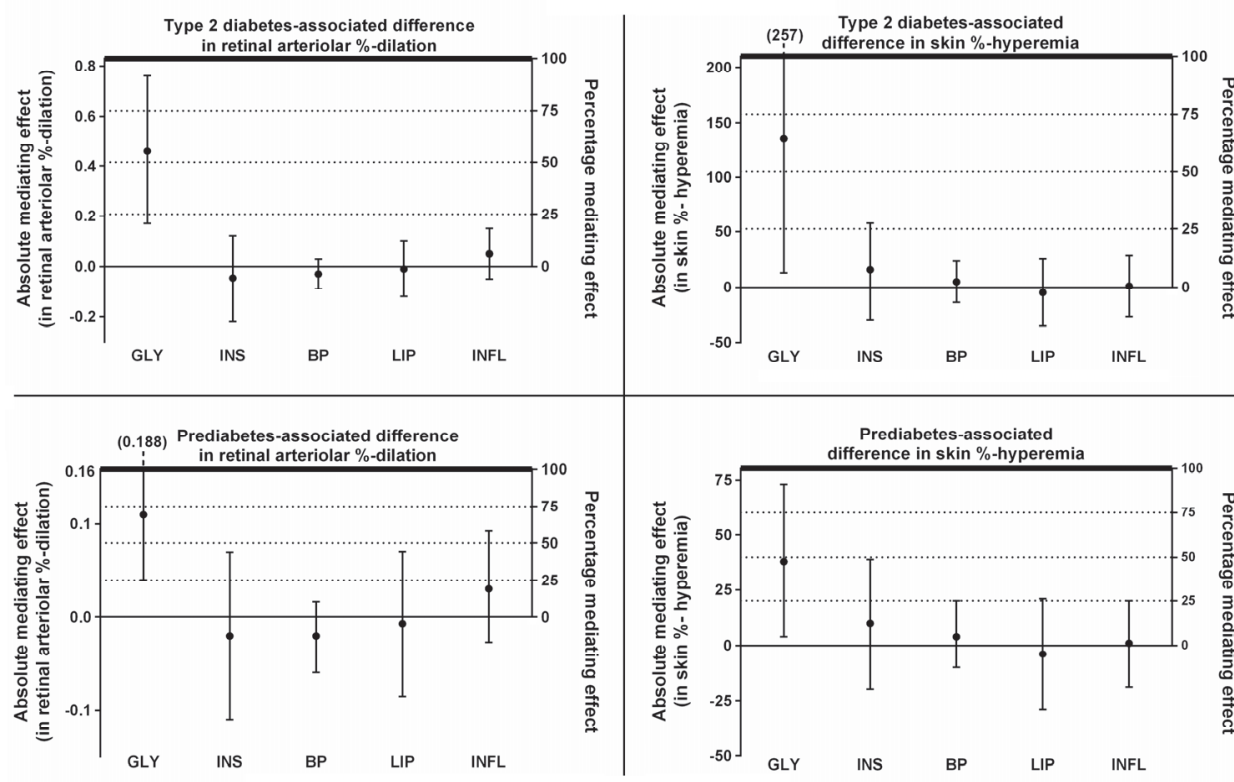

Figure 3.3 Fully-adjusted mediating effects of composite indices on the difference in retinal arteriolar \%-dilation (left) and skin \%-hyperemia (right) between individuals with type 2 diabetes and normal glucose metabolism (top) and individuals with prediabetes and normal glucose metabolism (bottom).

Mediating effects are presented as the indirect effects of type 2 diabetes (top) or prediabetes (bottom) on retinal arteriolar \%-dilation (left) and skin \%-hyperemia (right) through the potential mediators in \%-microvascular function [left Y-axis] and percentage of mediation [right $\mathrm{Y}$-axis]. Composite indices of potential mediators are adjusted for each other and for age and sex. The thick horizontal line indicates the mean difference in retinal arteriolar \%-dilation (left) or skin $\%$-hyperemia (right) between individuals with type 2 diabetes and those with normal glucose metabolism (top) or individuals with prediabetes and normal glucose metabolism (bottom), and thus corresponds with a mediation effect of $100 \%$. Abbreviations as in Figure 3.2.

\section{Discussion}

This study demonstrates that prediabetes- and T2D-associated impaired retinal and skin microvascular function were explained mainly by hyperglycemia. In contrast, insulin resistance, blood pressure, arterial stiffness, lipid profile, and low-grade inflammation did not significantly contribute. Comparable results were obtained after adjustment for body mass index and smoking status, history of cardiovascular disease, retinopathy, eGFR, and urinary albumin excretion. These findings suggest that hyperglycemia itself, rather than the cardiovascular risk context in which diabetes typically develops, is the 
main contributor to the differences in retinal and skin microvascular function between individuals with prediabetes or relatively well-controlled T2D and individuals with NGM.

Analyses were performed with multiple mediation instead of single mediation or multiple regression models, as they provide a superior reflection of the causal chain one tries to investigate ${ }^{28}$. Multiple mediation analyses enabled us to determine to what extent specific composite indices of potential mediators explained prediabetes- and T2D-associated retinal and skin microvascular dysfunction. Note that mediation and association represent different concepts. An association reflects a relationship between two variables, whereas a mediating effect indicates whether an association between two variables is explained (partly) by a third variable/mediator (Supplemental Figure S3.1) ${ }^{28}$.

Earlier studies have focused mainly on whether the effects of prediabetes and T2D on microvascular function were independent of cardiovascular risk factors, including blood pressure and lipid profile ${ }^{7,29}$. However, because markers of hyperglycemia, insulin resistance, blood pressure, lipid profile, and low-grade inflammation have been shown to be associated with microvascular dysfunction, irrespective of glucose metabolism status $^{11,21,22}$, the question arises to what extent such metabolic and vascular factors may explain (pre)diabetes-associated microvascular dysfunction. In this context, this study shows that hyperglycemia, irrespective of insulin resistance, blood pressure, lipid profile, and low-grade inflammation is the main mediator of prediabetes- and T2D-associated retinal and skin microvascular dysfunction, with a mediation effect of approximately 50-75\% (Figure 3.3). Our finding of a similar pattern of mediation, by hyperglycemia, of both prediabetes- and T2D-associated retinal and skin microvascular dysfunction supports an early detrimental effect of hyperglycemia on the retinal and skin microvasculature. The mechanism linking hyperglycemia and microvascular dysfunction may be bidirectional, as hyperglycemia may be both cause and consequence of microvascular dysfunction ${ }^{23}$. Impairments in flicker light-induced retinal arteriolar dilation and heat-induced skin hyperemia both depend (partly) on nitric oxide and are likely a reflection of microvascular endothelial dysfunction ${ }^{30,31}$, possibly in conjunction with neuronal dysfunction ${ }^{32,33}$. Pathophysiologically, hyperglycemia can impair microvascular endothelial function through intraendothelial accumulation of glucose, formation of advanced glycation end products, and increased oxidative stress ${ }^{34}$. In addition, microvascular endothelial dysfunction can cause hyperglycemia by impairing insulin secretion ${ }^{35}$ and by impairing the timely access of glucose and insulin to their target tissue ${ }^{36}$. Consequently, a vicious circle may exist between hyperglycemia and microvascular endothelial dysfunction ${ }^{23}$.

The composite index of blood pressure did not significantly explain the (pre)diabetesassociated difference in retinal and skin microvascular function. However, sensitivity analyses showed that the use of antihypertensive medication, and to a lesser extent 
diastolic blood pressure, explained 2.8 to $15.3 \%$ of T2D-associated retinal arteriolar dysfunction. The use of antihypertensive medication may be a reflection of previous exposure to elevated blood pressure, which may have affected retinal arteriolar function more than actual blood pressure. Similar results as for antihypertensive medication were found when we further specified antihypertensive medication into renin-angiotensinaldosterone system (RAAS)-inhibiting (with or without other types of antihypertensives) and non-RAAS-inhibiting antihypertensives only. In addition, the frequent use of antihypertensive medication among individuals with T2D (70.1\%) may have resulted in a small difference $(<5 \mathrm{mmHg}$ ) in actual 24-h blood pressure between individuals without and with T2D. Therefore, the possibility to assess a mediating effect of actual blood pressure in diabetes-associated retinal and skin microvascular dysfunction may have been limited.

(Pre)diabetes-associated retinal and skin microvascular dysfunction was not significantly explained by indices of insulin resistance, lipid profile, arterial stiffness, and low-grade inflammation. However, these findings need to be interpreted with caution. First, the index of insulin resistance we used is likely to be less accurate than those of hyperglycemia and blood pressure. Second, the frequent use of lipid-modifying medication among individuals with T2D (73.7\%) probably resulted in lower LDL-cholesterol in individuals with T2D as compared to NGM, which may have limited the possibility to assess a mediating effect of lipid profile in diabetes-associated microvascular dysfunction. Third, the absence of significant mediation by arterial stiffness of, especially, (pre)diabetes-associated retinal microvascular dysfunction was somewhat unexpected, as the retina is known to be sensitive to the higher flow pulsatility associated with arterial stiffening ${ }^{37}$. Possibly, the difference in arterial stiffness between individuals without and with (pre)diabetes was too small in this relatively well-treated population ${ }^{38}$. Finally, diabetes-associated retinal arteriolar dysfunction was, to a limited extent ( $7.5 \%$ ), explained by interleukin- 6 levels, but this may be an underestimation related to the use of a single systemic measurement of a biomarker, instead of local measurement ${ }^{39}$.

In conclusion, we did not find strong evidence for mediating roles of insulin resistance, blood pressure, arterial stiffening, lipid profile, or low-grade inflammation but cannot exclude the possibility that such mediation effects do in fact exist, especially in populations with greater differences in cardiovascular risk profile between individuals without and with diabetes.

Strengths of our study include its population-based design with oversampling of individuals with $\mathrm{T} 2 \mathrm{D}$, which enables an accurate comparison of individuals without and with T2D; the extensive assessment of potential mediators, including 24-h ambulatory blood pressure; the use of two independent methods to directly assess microvascular 
function; the multiple mediation analysis, which offers the possibility to compare competing mediators against one another ${ }^{28}$; and the broad array of additional analyses (Supplemental Material), which all gave consistent results.

Our study had some limitations. First, the data were cross-sectional; therefore we cannot exclude reverse causality. While we acknowledge this limitation, there is a strong a priori likelihood from the association between diabetes and microangiopathy that hyperglycemia can cause microvascular dysfunction; however the association may be bidirectional $^{23}$. Second, although we considered many potential mediators, we cannot exclude residual mediation by variables not included in these analyses (e.g. dietary habits and physical activity). We therefore do not claim to have identified all contributors to (pre)diabetes-associated microvascular dysfunction. Third, the statistical power of the hyperglycemia index may have been decreased due to autocorrelation between measures of hyperglycemia and glucose metabolism status ${ }^{40}$, leading to the wide $95 \% \mathrm{Cls}$ around its mediation effect. However, results for hyperglycemia were qualitatively similar when the index was composed of measures that were not directly used for diabetes classification (Supplemental Figure S3.5). In addition, collinearity diagnostics were negative. Fourth, we did not correct for multiple testing, which may have increased the possibility of a false positive finding. However, it is unlikely that the pattern of mediation we observed, and that remained consistent across multiple sensitivity analyses, is only explained by chance. Fifth, generalizability of our results should be interpreted with caution, as in our cohort individuals with T2D were generally well controlled for their diabetes and cardiovascular risk factors. However, selection bias has not occurred, as individuals were not included based on their glycemic or vascular risk factor control. Our population may therefore be representative for a population that has access to quality diabetes care.

In summary, we showed, in a population-based study, that prediabetes- and T2D-associated retinal and skin microvascular dysfunction were explained mainly by hyperglycemia. In this population insulin resistance, arterial stiffness, lipid profile, and low-grade inflammation did not explain prediabetes- and T2D-associated retinal and skin microvascular dysfunction. These findings suggest early and intensive glycemic control in prediabetes and $\mathrm{T} 2 \mathrm{D}$ as a promising therapeutic target for the prevention of (pre)diabetes-associated microvascular complications. 


\section{References}

1. Guariguata L, Whiting DR, Hambleton I, Beagley J, Linnenkamp U and Shaw JE. Global estimates of diabetes prevalence for 2013 and projections for 2035. Diabetes Res Clin Pract. 2014;103:137-49.

2. Forouhi NG and Wareham NJ. Epidemiology of diabetes. Medicine. 2014;42:698-702.

3. Stratton IM, Adler AI, Neil HA, Matthews DR, Manley SE, Cull CA, Hadden D, Turner RC and Holman RR. Association of glycaemia with macrovascular and microvascular complications of type 2 diabetes (UKPDS 35): prospective observational study. BMJ. 2000;321:405-12.

4. Lee JF, Barrett-O'Keefe Z, Garten RS, Nelson AD, Ryan JJ, Nativi JN, Richardson RS and Wray DW. Evidence of microvascular dysfunction in heart failure with preserved ejection fraction. Heart. 2016;102:278-84.

5. van Sloten TT, Schram MT, Adriaanse MC, Dekker JM, Nijpels G, Teerlink T, Scheffer PG, Pouwer F, Schalkwijk CG, Stehouwer CD and Henry RM. Endothelial dysfunction is associated with a greater depressive symptom score in a general elderly population: the Hoorn Study. Psychol Med. 2014;44: 1403-16.

6. Buysschaert M, Medina JL, Bergman M, Shah A and Lonier J. Prediabetes and associated disorders. Endocrine. 2015;48:371-93.

7. Sörensen BM, Houben AJ, Berendschot TT, Schouten JS, Kroon AA, van der Kallen CJ, Henry RM, Koster A, Sep SJ, Dagnelie PC, Schaper NC, Schram MT and Stehouwer CD. Prediabetes and Type 2 Diabetes Are Associated With Generalized Microvascular Dysfunction: The Maastricht Study. Circulation. 2016;134:1339-52.

8. Wong MS, Gu K, Heng D, Chew SK, Chew LS and Tai ES. The Singapore impaired glucose tolerance followup study: does the ticking clock go backward as well as forward? Diabetes Care. 2003;26:3024-30.

9. Mitchell GF, Vita JA, Larson MG, Parise H, Keyes MJ, Warner E, Vasan RS, Levy D and Benjamin EJ. Cross-sectional relations of peripheral microvascular function, cardiovascular disease risk factors, and aortic stiffness: the Framingham Heart Study. Circulation. 2005;112:3722-8.

10. De Boer MP, Meijer RI, Wijnstok NJ, Jonk AM, Houben AJ, Stehouwer CD, Smulders YM, Eringa EC and Serne EH. Microvascular dysfunction: a potential mechanism in the pathogenesis of obesity-associated insulin resistance and hypertension. Microcirculation. 2012;19:5-18.

11. Shore AC and Tooke JE. Microvascular function in human essential hypertension. J Hypertens. 1994;12:717-28.

12. Serne EH, Stehouwer CD, ter Maaten JC, ter Wee PM, Rauwerda JA, Donker AJ and Gans RO. Microvascular function relates to insulin sensitivity and blood pressure in normal subjects. Circulation. 1999;99:896-902.

13. Serne EH, Gans RO, ter Maaten JC, ter Wee PM, Donker AJ and Stehouwer CD. Capillary recruitment is impaired in essential hypertension and relates to insulin's metabolic and vascular actions. Cardiovasc Res. 2001;49:161-8.

14. Kim JA, Montagnani M, Koh KK and Quon MJ. Reciprocal relationships between insulin resistance and endothelial dysfunction: molecular and pathophysiological mechanisms. Circulation. 2006;113:1888-904.

15. Soro-Paavonen A, Zhang WZ, Venardos K, Coughlan MT, Harris E, Tong DC, Brasacchio D, Paavonen K, Chin-Dusting J, Cooper ME, Kaye D, Thomas MC and Forbes JM. Advanced glycation end-products induce vascular dysfunction via resistance to nitric oxide and suppression of endothelial nitric oxide synthase. J Hypertens. 2010;28:780-8.

16. Clark MG. Impaired microvascular perfusion: a consequence of vascular dysfunction and a potential cause of insulin resistance in muscle. Am J Physiol Endocrinol Metab. 2008;295:E732-50.

17. Hajer GR, van Haeften TW and Visseren FLJ. Adipose tissue dysfunction in obesity, diabetes, and vascular diseases. Eur Heart J. 2008;29:2959-71.

18. Anderson RA, Evans ML, Ellis GR, Graham J, Morris K, Jackson SK, Lewis MJ, Rees A and Frenneaux MP. The relationships between post-prandial lipaemia, endothelial function and oxidative stress in healthy individuals and patients with type 2 diabetes. Atherosclerosis. 2001;154:475-83.

19. Lonati C, Morganti A, Comarella L, Mancia G, Zanchetti A and Group IS. Prevalence of type 2 diabetes among patients with hypertension under the care of 30 Italian clinics of hypertension: results of the (Iper)tensione and (dia)bete study. J Hypertens. 2008;26:1801-8. 
20. Schram MT, Henry RM, van Dijk RA, Kostense PJ, Dekker JM, Nijpels G, Heine RJ, Bouter LM, Westerhof N and Stehouwer CD. Increased central artery stiffness in impaired glucose metabolism and type 2 diabetes - The Hoorn study. Hypertension. 2004;43:176-81.

21. Reimann $\mathrm{M}$, Weiss $\mathrm{N}$ and Ziemssen $\mathrm{T}$. Different responses of the retinal and cutaneous microcirculation to transient dysmetabolic conditions. Atherosclerosis Supp. 2015;18:1-7.

22. Cheng $C$ and Daskalakis C. Association of Adipokines with Insulin Resistance, Microvascular Dysfunction, and Endothelial Dysfunction in Healthy Young Adults. Mediators Inflamm. 2015;2015:594039.

23. Muris DM, Houben AJ, Schram MT and Stehouwer CD. Microvascular dysfunction: an emerging pathway in the pathogenesis of obesity-related insulin resistance. Rev Endocr Metab Disord. 2013;14:29-38.

24. Nguyen TT, Kreis AJ, Kawasaki R, Wang JJ, Seifert BU, Vilser W, Nagel E and Wong TY. Reproducibility of the retinal vascular response to flicker light in Asians. Curr Eye Res. 2009;34:1082-8.

25. Agarwal SC, Allen J, Murray A and Purcell IF. Comparative reproducibility of dermal microvascular blood flow changes in response to acetylcholine iontophoresis, hyperthermia and reactive hyperaemia. Physiol Meas. 2010;31:1-11.

26. Schram MT, Sep SJ, van der Kallen CJ, Dagnelie PC, Koster A, Schaper NC, Henry RM and Stehouwer CD. The Maastricht Study: an extensive phenotyping study on determinants of type 2 diabetes, its complications and its comorbidities. Eur J Epidemiol. 2014;29:439-51.

27. van Bussel BC, Henry RM, Schalkwijk CG, Ferreira I, Feskens EJ, Streppel MT, Smulders YM, Twisk JW and Stehouwer CD. Fish consumption in healthy adults is associated with decreased circulating biomarkers of endothelial dysfunction and inflammation during a 6-year follow-up. J Nutr. 2011;141:1719-25.

28. Preacher KJ and Hayes AF. Asymptotic and resampling strategies for assessing and comparing indirect effects in multiple mediator models. Behav Res Methods. 2008;40:879-91.

29. Nguyen TT, Kawasaki R, Wang JJ, Kreis AJ, Shaw J, Vilser W and Wong TY. Flicker light-induced retinal vasodilation in diabetes and diabetic retinopathy. Diabetes Care. 2009;32:2075-80.

30. Kellogg DL, Jr., Liu Y, Kosiba IF and O'Donnell D. Role of nitric oxide in the vascular effects of local warming of the skin in humans. J Appl Physiol (1985). 1999;86:1185-90.

31. Lim M, Sasongko MB, Ikram MK, Lamoureux E, Wang JJ, Wong TY and Cheung CY. Systemic associations of dynamic retinal vessel analysis: a review of current literature. Microcirculation. 2013;20:257-68.

32. Falsini B, Riva CE and Logean E. Flicker-evoked changes in human optic nerve blood flow: relationship with retinal neural activity. Invest Ophthalmol Vis Sci. 2002;43:2309-16.

33. Minson CT, Berry LT and Joyner MJ. Nitric oxide and neurally mediated regulation of skin blood flow during local heating. J Appl Physiol (1985). 2001;91:1619-26.

34. Chilelli NC, Burlina S and Lapolla A. AGEs, rather than hyperglycemia, are responsible for microvascular complications in diabetes: a "glycoxidation-centric" point of view. Nutr Metab Cardiovasc Dis. 2013;23:913-9.

35. Hashimoto S, Kubota N, Sato H, Sasaki M, Takamoto I, Kubota T, Nakaya K, Noda M, Ueki K and Kadowaki T. Insulin receptor substrate-2 (Irs2) in endothelial cells plays a crucial role in insulin secretion. Diabetes. 2015;64:876-86.

36. Jonk AM, Houben AJ, de Jongh RT, Serne EH, Schaper NC and Stehouwer CD. Microvascular dysfunction in obesity: a potential mechanism in the pathogenesis of obesity-associated insulin resistance and hypertension. Physiology. 2007;22:252-60.

37. Cheung N, Sharrett AR, Klein R, Criqui MH, Islam FM, Macura KJ, Cotch MF, Klein BE and Wong TY. Aortic distensibility and retinal arteriolar narrowing: the multi-ethnic study of atherosclerosis. Hypertension. 2007;50:617-22.

38. Geijselaers SL, Sep SJ, Schram MT, van Boxtel MP, van Sloten TT, Henry RM, Reesink KD, Kroon AA, Koster A, Schaper NC, Dagnelie PC, van der Kallen CJ, Biessels GJ and Stehouwer CD. Carotid stiffness is associated with impairment of cognitive performance in individuals with and without type 2 diabetes. The Maastricht Study. Atherosclerosis. 2016;253:186-93.

39. Koskela UE, Kuusisto SM, Nissinen AE, Savolainen MJ and Liinamaa MJ. High vitreous concentration of IL-6 and IL-8, but not of adhesion molecules in relation to plasma concentrations in proliferative diabetic retinopathy. Ophthalmic Res. 2013;49:108-14.

40. Beasley TM. Tests of Mediation: Paradoxical Decline in Statistical Power as a Function of Mediator Collinearity. J Exp Educ. 2014;82:283-306. 


\section{Supplemental Material to chapter 3}

\section{Supplemental Methods}

\section{Study population}

The Maastricht Study ${ }^{1}$ focuses on the etiology, pathophysiology, complications, and comorbidities of type 2 diabetes mellitus (T2D) and is characterized by an extensive phenotyping approach. Eligible for participation were all individuals aged between 40 and 75 years and living in the southern part of the Netherlands. The examinations of each participant were performed within a time window of three months. Participants were recruited through mass media campaigns and from the municipal registries and the regional Diabetes Patient Registry via mailings. Recruitment was stratified according to known T2D status, with an oversampling of individuals with T2D, for reasons of efficiency.

\section{Glucose metabolism status}

To assess glucose metabolism status, all participants (except those who used insulin) underwent a standardized 2-h 75 gram oral glucose tolerance test (OGTT) after an overnight fast. For safety reasons, participants with a fasting glucose level above $11.0 \mathrm{mmol} / \mathrm{l}$, as determined by a finger prick, did not undergo the OGTT. For these individuals fasting glucose level and information about diabetes medication use were used to assess glucose metabolism status. Glucose metabolism status was defined according to the World Health Organization 2006 criteria as normal glucose metabolism, impaired fasting glucose, impaired glucose tolerance (combined as prediabetes), or T2D $\mathrm{D}^{1}$.

\section{Assessment of retinal and skin microvascular function}

All participants were asked to refrain from smoking and drinking caffeine-containing beverages three hours before the measurement. A light meal (breakfast and (or) lunch), low in fat content, was allowed at last 90 minutes prior to the start of the measurements.

\section{Retinal arteriolar dilation response $e^{2}$}

For retinal measurements pupils were dilated with $0.5 \%$ tropicamide and $2.5 \%$ phenylephrine at least 15 minutes prior to the start of the examination. The retinal arteriolar dilation response to flicker light was measured in a dimly lit room by use of the Dynamic Vessel Analyzer (DVA) (Imedos, Jena, Germany). For safety reasons, participants 
with an intraocular pressure exceeding $30 \mathrm{mmHg}$ were excluded from retinal measurements. Per participant, we randomly measured the left or right eye.

During the measurement, the participant was instructed and encouraged to focus on the tip of a fixated needle inside the retinal camera (FF450; Carl Zeiss GmbH, Jena, Germany), while the fundus of the eye was examined under green measuring light (530-600 nm, illumination of fundus approximately 6500 lux). A straight arteriolar segment of approximately $1.5 \mathrm{~mm}$ in length located 0.5 to 2.0 disc diameter from the margin of the optic disc in the temporal section was examined. When the specific vessel profile was recognized, vessel diameter was automatically and continuously measured for 150 seconds. A baseline recording of 50 seconds was followed by a 40-second flicker light exposure period (flicker frequency $12.5 \mathrm{~Hz}$, bright-to-dark contrast ratio 25:1) followed by a 60-second recovery period. The DVA automatically corrected for alterations in luminance caused by, for example, slight eye movements. During blinks and small eye movements, the registration stopped and restarted once the vessel segment was automatically re-identified.

The integrated DVA software (version 4.51, Imedos) automatically calculated baseline diameter and percentage dilation. Baseline diameter was calculated as the average diameter size of the 20-50 seconds recording and was expressed in measurement units (MU), where $1 \mathrm{MU}$ is equal to $1 \mu \mathrm{m}$ of the Gullstrand eye $\mathrm{e}^{3}$. Percentage dilation over baseline was based on the average dilation achieved at time points 10 and 40 seconds during the flicker stimulation period. The purpose of taking the average dilation was to account for inter-individual variation in the curve shape during dilation.

\section{Skin hyperemic response $e^{2}$}

Skin blood flow measurements were performed in a climate-controlled room at $24^{\circ} \mathrm{C}$ with participants in a supine position. Skin blood flow was measured as described previously by means of a laser-Doppler system (Periflux 5000, Perimed, Järfälla, Sweden), equipped with a thermostatic laser-Doppler probe (PF457) at the dorsal side of the wrist of the left hand ${ }^{4}$. The laser-Doppler output was recorded for 25 minutes with a sample rate of $32 \mathrm{~Hz}$, which gives semi-quantitative assessment of skin blood flow expressed in arbitrary perfusion units (PU). Skin blood flow was first recorded unheated for 2 minutes to serve as a baseline. After the 2 minutes of baseline, the temperature of the probe was rapidly and locally increased to $44^{\circ} \mathrm{C}$, and was then kept constant until the end of the registration. The heat-induced skin hyperemic response was expressed as the percentage increase in average PU during the 23 minutes heating phase over the average baseline PU. 


\section{Assessment of potential mediators}

\section{Hyperglycemia}

Venous fasting and postload plasma glucose levels were measured by the enzymatic hexokinase method on two automatic analyzers (i.e. the Beckman Synchron LX20 (Beckman Coulter Inc., USA) for samples obtained between November 2010 and April 2012, and the Roche Cobas 6000 (Roche Diagnostics, Mannheim, Germany) for samples obtained thereafter). Glycated hemoglobin A1c (HbA1c) was determined by ionexchange high-performance liquid chromatography ${ }^{1}$. Skin auto-fluorescence as a marker of tissue advanced glycation end product accumulation was measured with the AGE reader (DiagnOptics Technologies B.V., Groningen, the Netherlands). Details on the AGE reader measurements, and data on their reproducibility, have been published previously 5 .

\section{Insulin resistance}

Homeostasis model assessment of insulin resistance (HOMA2-IR) insulin and HOMA2-IR $\mathrm{R}_{\mathrm{C} \text {-peptide }}$ were both calculated by use of the HOMA2-calculator version 2.2.3 (available from https://www.dtu.ox.ac.uk/homacalculator). HOMA2-IR is a widely used surrogate marker of insulin resistance and corresponds reasonably well to clamp-derived measures of insulin resistance ${ }^{6}$. HOMA2-IR $\mathrm{R}_{\mathrm{c} \text {-peptide }}$ may be a better marker of peripheral insulin resistance than $\mathrm{HOMA2}-\mathrm{IR}_{\text {insulin, }}$ as $\mathrm{C}$-peptide is not cleared by the liver, and is therefore considered a more stable and thus accurate marker of endogenous insulin secretion. Fasting insulin and C-peptide levels were both quantified on a Meso Scale custom duplex assay (Meso Scale Discovery, Gaithersburg, Maryland, USA).

\section{Blood pressure}

Participants were asked to undergo 24-h blood pressure monitoring (WatchBP03, Microlife AG, Widnau, Switzerland) with an appropriate-sized cuff placed around their non-dominant $\operatorname{arm}^{1,7}$. The device was programmed to take blood pressure readings every 15 minutes during daytime (8 a.m. - 11 p.m.) and every 30 minutes during the night (11 p.m. -8 a.m.). According to recommendations of the British Hypertension society $^{8}$, mean $24-h$ blood pressure measurements were only calculated if there were more than 14 valid blood pressure measurements during daytime and more than 7 valid measurements during nighttime. The use of antihypertensive medication was assessed during a medication interview where generic name, dose, and frequency were registered ${ }^{1}$. 


\section{Arterial stiffness}

Carotid-femoral pulse wave velocity was measured at the right common carotid and right common femoral artery, with the use of applanation tonometry (sphygmoCor, Atcor Medical, Sydney, Australia) according to recent guidelines ${ }^{9}$. Carotid diameter (D) and distension (DS) of the left common carotid artery (at least $10 \mathrm{~mm}$ proximal to the carotid bulb) were measured with an ultrasound scanner equipped with a $7.5 \mathrm{MHz}$ linear probe (MyLab 70, Esaote Europe B.V., Maastricht, the Netherlands). Combined with brachial pulse pressure, these measures were used to calculate the carotid distensibility coefficient with the formula: $\left(2 D S * D+D S^{2}\right) /\left(\right.$ pulse pressure $\left.* D^{2}\right)$. Note that higher values of carotid-femoral pulse wave velocity, but lower values of the carotid distensibility coefficient reflect greater arterial stiffness. Further details on the carotidfemoral pulse wave velocity, carotid distensibility index, and data on their reproducibility, have been published previously ${ }^{10}$.

\section{Lipid profile}

Serum concentrations of total cholesterol, high-density lipoprotein (HDL) cholesterol, and triglycerides were measured using an automatic analyzer (Beckman Synchron LX20, Beckman Coulter Inc. Brea, USA). The use of lipid-modifying medication was assessed during a medication interview where generic name, dose, and frequency were registered ${ }^{1}$.

\section{Markers of low-grade inflammation}

Plasma biomarkers of low-grade inflammation (high-sensitive C-reactive protein (hs-CRP), serum amyloid A (SAA), soluble intercellular adhesion molecule-1 (sICAM-1), interleukin-6, interleukin-8, and tumor necrosis factor- $\alpha$ (TNF- $\alpha)$ ) of the first 866 individuals of The Maastricht Study were measured in ethylenediaminetetraacetic acid (EDTA) plasma samples with commercially available 4-plex sandwich immunoassay kits (Meso Scale Discovery, Rockville, Maryland, USA) as described previously ${ }^{11}$.

From individual 867 onwards, plasma biomarkers of low-grade inflammation were measured in EDTA plasma samples with renewed commercially available 4-plex sandwich immunoassay kits with different standards and antibodies (Meso Scale Discovery, Rockville, Maryland, USA). For this technique in this study, the intra- and inter-assay coefficients of variation were 5.4 and 5.4 for hs-CRP, 8.7 and 10.8 for SAA, 10.3 and 8.4 for sICAM-1, 13.2 and 11.9 for interleukin-6, 7.6 and 5.5 for interleukin-8, and 4.3 and 6.2 for TNF- $\alpha$, respectively. 
Absolute values of plasma biomarkers of low-grade inflammation differed between the individuals measured with the old and renewed 4-plex sandwich immunoassay kits. To realign the absolute low-grade inflammation marker values of individuals measured with the old 4-plex sandwich immunoassay to individuals measured with the renewed 4-plex sandwich immunoassay, realign formulas were calculated with Deeming regression analyses ${ }^{12}$. In order to do so, the first 419 out of 866 individuals, who were measured with the old 4-plex sandwich immunoassay, were measured with the renewed 4-plex sandwich immunoassay as well.

\section{Assessment of covariates}

History of cardiovascular disease, smoking status (never, former, current), and duration of diabetes were assessed by web-based questionnaires ${ }^{1}$. History of cardiovascular disease was defined as a history of myocardial infarction, stroke, or vascular surgery (including angioplasty) of coronary, carotid, abdominal aortic, or peripheral arteries. We measured height, weight, waist circumference, office blood pressure, serum creatinine and cystatin $\mathrm{C}^{13}$, and 24 -h urinary albumin excretion (twice) as described previously ${ }^{1}$. Body mass index was calculated as weight $(\mathrm{kg})$ divided by height $\left(\mathrm{m}^{2}\right)$. Estimated glomerular filtration rate (eGFR; in $\mathrm{ml} / \mathrm{min} / 1.73 \mathrm{~m}^{2}$ ) was calculated with the Chronic Kidney Disease Epidemiology Collaboration (CDK-epi) equation based on both serum creatinine and serum cystatin $C^{14}$. The presence of retinopathy was assessed in both eyes by use of fundus photographs taken with an auto fundus camera (Model AFC-230, Nidek, Gamagori, Japan) ${ }^{1}$.

\section{Statistical analysis}

Differences in general characteristics among individuals in the study populations and individuals excluded due to missing values were compared by Analyses of Variance (ANOVA) for continuous variables and $\chi^{2}$-test for categorical variables. 
A

\begin{tabular}{|c|c|c|}
\hline Type 2 diabetes & Total effect $c$ & Microvascular \\
\hline
\end{tabular}

B

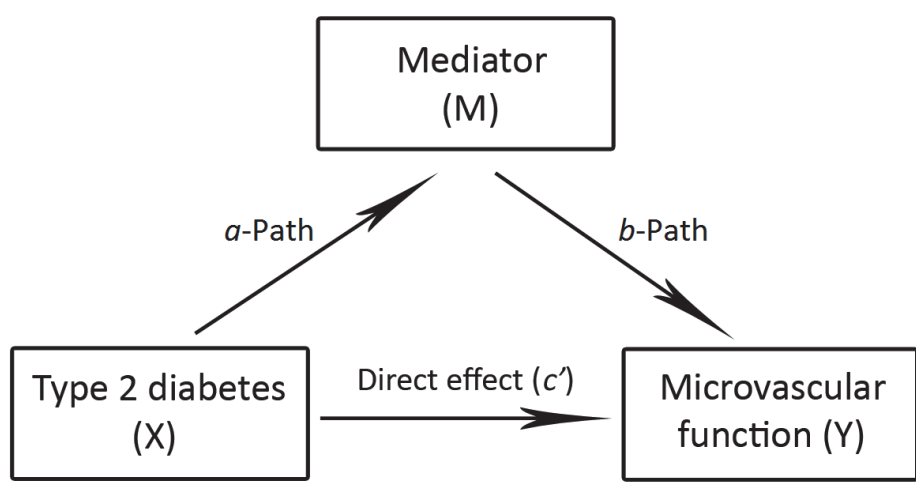

Figure S3.1 (A) Model showing the total effect $(c)$ of type 2 diabetes (X) on retinal or skin microvascular function (Y). (B) Illustration of a simple mediation design in which type 2 diabetes' $(X)$ causal effect on retinal or skin microvascular function $(Y)$ is hypothesized to exert an indirect effect (a-path *b-path) through each potential mediator $(\mathrm{M})$ and a remaining direct effect on $Y\left(\right.$ path $\left.c^{\prime}\right)$.

The $a$-path represents the effect of $X$ on the proposed mediator $(\mathrm{M})$, while the $b$-path represents the effect of $\mathrm{M}$ on $\mathrm{Y}$. The product of the $a$ - and $b$-path $(a * b)$ represents the indirect effect of $X$ on $Y$ via mediator $M$. The total effect $(c)$ of $X$ on $Y$ can be expressed as the sum of the indirect and direct effects: $c=c^{\prime}+a b$. Potential mediators $(M)$ are markers of hyperglycemia, insulin resistance, blood pressure, lipid profile, and low-grade inflammation. 

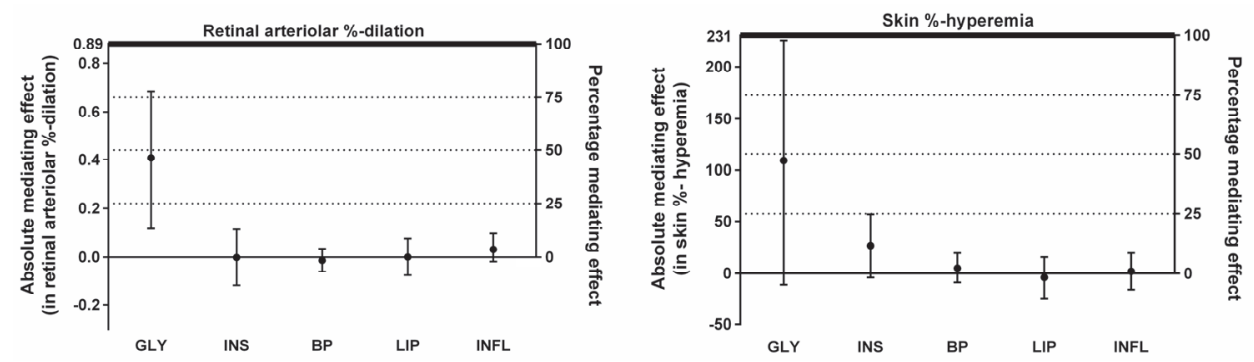

Figure S3.2 Mediating effects of composite indices on the difference in retinal arteriolar \%-dilation (left) and skin \%-hyperemia (right) between individuals with type 2 diabetes and normal glucose metabolism, with smoking status and body mass index as additional covariates.

Mediating effects are presented as the indirect effects of type 2 diabetes on retinal arteriolar \%-dilation (left) and skin \%-hyperemia (right) through the potential mediators in \%-microvascular function [left Y-axis] and percentage of mediation [right Y-axis]. Composite indices of potential mediators are adjusted for each other and for age, sex, smoking status, and body mass index. The thick horizontal line indicates the mean difference in retinal arteriolar \%-dilation (left) and skin \%-hyperemia (right) between individuals with type 2 diabetes and those with normal glucose metabolism, and thus corresponds with a mediation effect of $100 \%$. GLY $=$ composite index of fasting glucose, glycated hemoglobin $\mathrm{A} 1 \mathrm{c}(\mathrm{HbA} \mathrm{Cc})$, and skin auto-fluorescence for retinal arteriolar reactivity analyses, and composite index of fasting glucose, 2 -h postload glucose, $\mathrm{HbA} 1 \mathrm{c}$, and skin auto-fluorescence for skin reactivity analyses; INS = composite index of 2 homeostasis model assessment of insulin resistance, 1 based on fasting plasma insulin and fasting plasma glucose, and the other based on fasting C-peptide and fasting plasma glucose; $\mathrm{BP}=$ composite index that combined 24-h systolic and diastolic blood pressure with the use of antihypertensive medication; LIP = composite index that combined total cholesterol, inverse high-density lipoprotein cholesterol, and triglycerides with the use of lipid-modifying medication; INFL = composite index that combined high-sensitive C-reactive protein, serum amyloid $\mathrm{A}$, soluble intercellular adhesion molecule-1, interleukin-6, interleukin-8, and tumor necrosis factor- $\alpha$. 

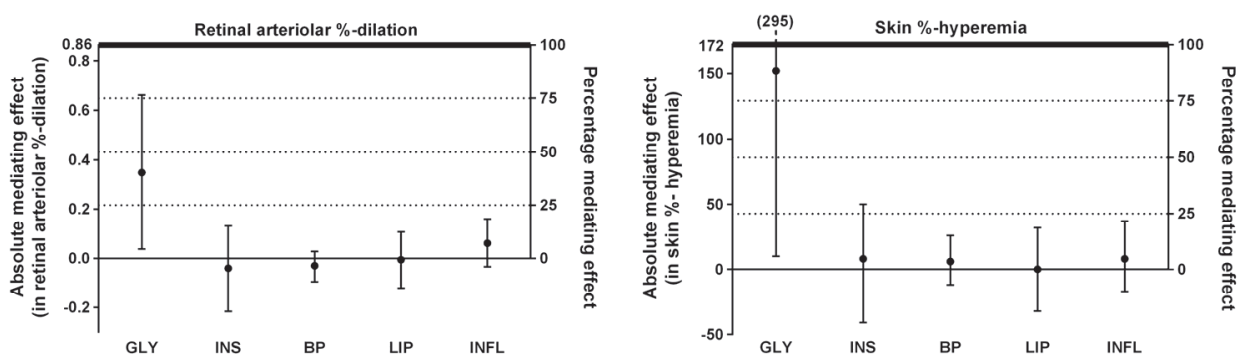

Figure S3.3 Mediating effects of composite indices on the difference in retinal arteriolar \%-dilation (left) and skin \%-hyperemia (right) between individuals with type 2 diabetes and normal glucose metabolism, with history of cardiovascular disease, retinopathy, estimated glomerular filtration rate, and urinary albumin excretion as additional covariates.

Mediating effects are presented as the indirect effects of type 2 diabetes on retinal arteriolar \%-dilation (left) and skin \%-hyperemia (right) through the potential mediators in \%-microvascular function [left Y-axis] and percentage of mediation [right $\mathrm{Y}$-axis]. Composite indices of potential mediators are adjusted for each other and for age, sex, history of cardiovascular disease, retinopathy, estimated glomerular filtration rate, and urinary albumin excretion (the latter 2 as continuous variables). The thick horizontal line indicates the mean difference in retinal arteriolar \%-dilation (left) and skin \%-hyperemia (right) between individuals with type 2 diabetes and those with normal glucose metabolism, and thus corresponds with a mediation effect of $100 \%$. Abbreviations as in Supplemental Figure S3.2.
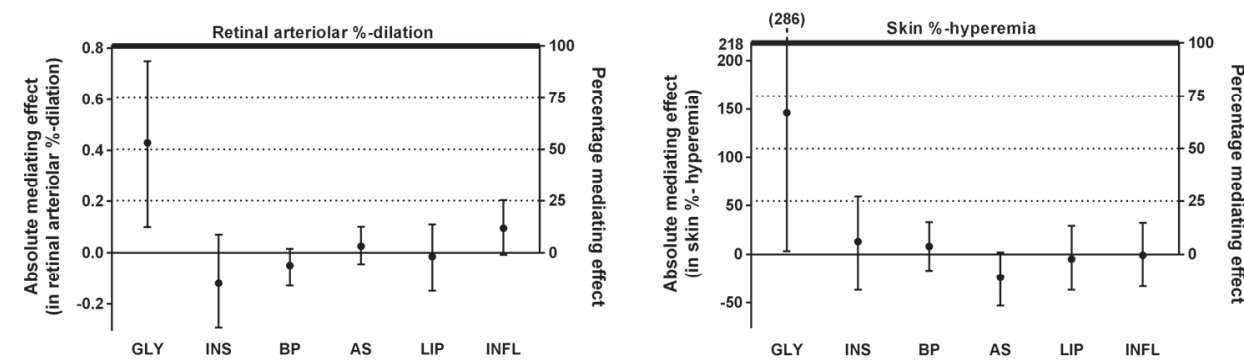

Figure S3.4 Mediating effects of composite indices with an additional composite index of arterial stiffness on the difference in retinal arteriolar \%-dilation (left) and skin \%-hyperemia (right) between individuals with type 2 diabetes and normal glucose metabolism.

Mediating effects are presented as the indirect effects of type 2 diabetes on retinal arteriolar \%-dilation (left) and skin \%-hyperemia (right) through the potential mediators in \%-microvascular function [left Y-axis] and percentage of mediation [right Y-axis]. Composite indices of potential mediators are adjusted for each other and for age and sex. The thick horizontal line indicates the mean difference in retinal arteriolar \%-dilation (left) and skin \%-hyperemia (right) between individuals with type 2 diabetes and those with normal glucose metabolism, and thus corresponds with a mediation effect of $100 \%$. AS = composite index that combined carotidfemoral pulse wave velocity and the inverse carotid distensibility coefficient. Abbreviations as in Supplemental Figure S3.2. 

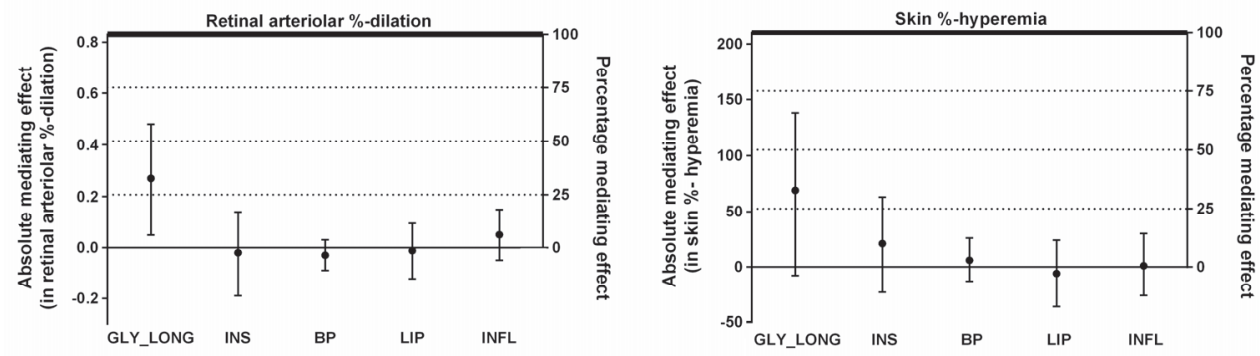

Figure S3.5 Mediating effects of composite indices on the difference in retinal arteriolar \%-dilation (left) and skin \%-hyperemia (right) between individuals with type 2 diabetes and normal glucose metabolism, with an alternatively-formed long-term hyperglycemia composite index.

Mediating effects are presented as the indirect effects of type 2 diabetes on retinal arteriolar \%-dilation (left) and skin \%-hyperemia (right) through the potential mediators in \%-microvascular function [left Y-axis] and percentage of mediation [right Y-axis]. Composite indices of potential mediators are adjusted for each other and for age and sex. The thick horizontal line indicates the mean difference in retinal arteriolar \%-dilation (left) and skin \%-hyperemia (right) between individuals with type 2 diabetes and those with normal glucose metabolism, and thus corresponds with a mediation effect of $100 \%$. GLY_LONG = composite index of HbA1c and skin auto-fluorescence (advanced glycation end products). Abbreviations as in Supplemental Figure S3.2.
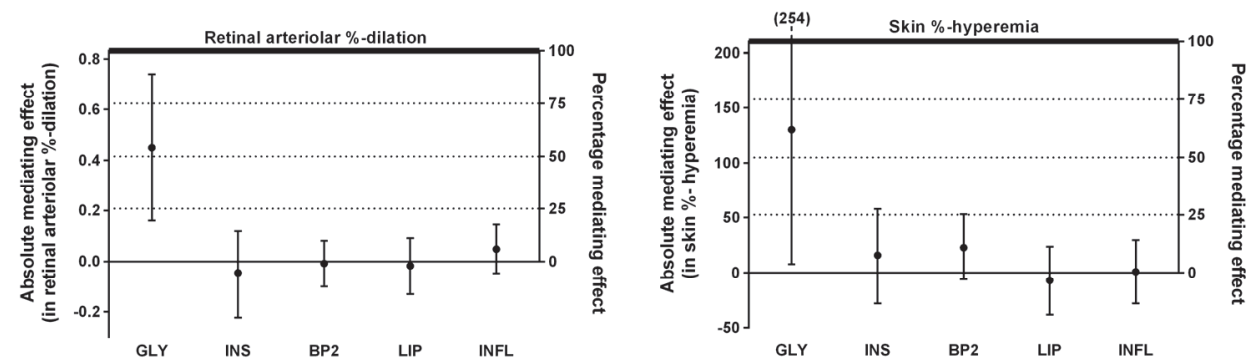

Figure S3.6 Mediating effects of composite indices on the difference in retinal arteriolar \%-dilation (left) and skin \%-hyperemia (right) between individuals with type 2 diabetes and normal glucose metabolism, with an alternatively-formed blood pressure composite index.

Mediating effects are presented as the indirect effects of type 2 diabetes on retinal arteriolar \%-dilation (left) and skin \%-hyperemia (right) through the potential mediators in \%-microvascular function [left $\mathrm{Y}$-axis] and percentage of mediation [right $\mathrm{Y}$-axis]. Composite indices of potential mediators are adjusted for each other and for age and sex. The thick horizontal line indicates the mean difference in retinal arteriolar \%-dilation (left) and skin \%-hyperemia (right) between individuals with type 2 diabetes and those with normal glucose metabolism, and thus corresponds with a mediation effect of $100 \%$. BP2 $=$ composite index that combined 24-h mean arterial pressure and 24-h pulse pressure with the use of antihypertensive medication. Abbreviations as in Supplemental Figure S3.2. 

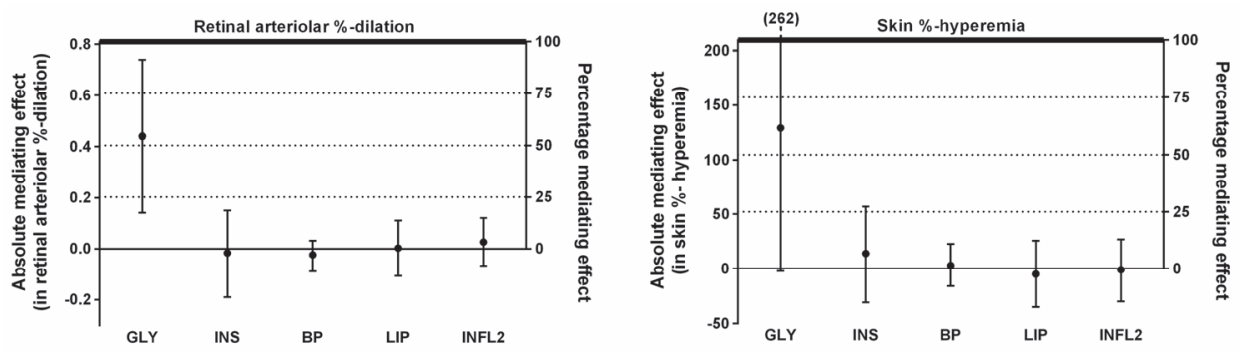

Figure S3.7 Mediating effects of composite indices on the difference in retinal arteriolar \%-dilation (left) and skin \%-hyperemia (right) between individuals with type 2 diabetes and normal glucose metabolism after exclusion of participants with high-sensitive C-reactive protein $>10 \mathrm{mg} / \mathrm{l}$.

Mediating effects are presented as the indirect effects of type 2 diabetes on retinal arteriolar \%-dilation (left) and skin \%-hyperemia (right) through the potential mediators in \%-microvascular function [left $\mathrm{Y}$-axis] and percentage of mediation [right $\mathrm{Y}$-axis]. Composite indices of potential mediators are adjusted for each other and for age and sex. The thick horizontal line indicates the mean difference in retinal arteriolar \%-dilation (left) and skin \%-hyperemia (right) between individuals with type 2 diabetes and those with normal glucose metabolism, and thus corresponds with a mediation effect of $100 \%$. INFL2 = composite index that combined highsensitive $\mathrm{C}$-reactive protein (after exclusion of participants with high-sensitive C-reactive protein $>10 \mathrm{mg} / \mathrm{l}$ ), serum amyloid A, soluble intercellular adhesion molecule-1, interleukin-6, interleukin-8, and tumor necrosis factor- $\alpha$. Abbreviations as in Supplemental Figure S3.2.
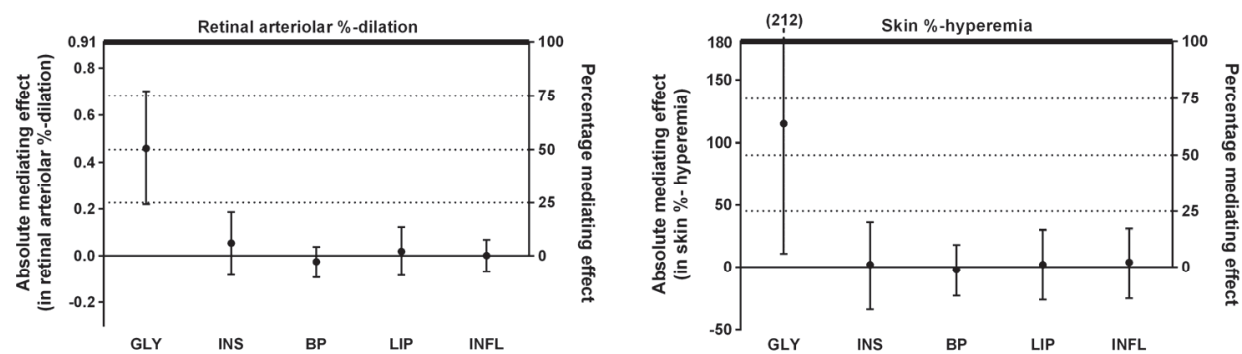

Figure S3.8 Mediating effects of composite indices on the difference in retinal arteriolar \%-dilation (left) and skin \%-hyperemia (right) between individuals with type 2 diabetes and normal glucose metabolism, with composite indices calculated when participants had data on at least one of the individual markers of which the composite indices consisted.

Mediating effects are presented as the indirect effects of type 2 diabetes on retinal arteriolar \%-dilation (left) and skin \%-hyperemia (right) through the potential mediators in \%-microvascular function [left Y-axis] and percentage of mediation [right Y-axis]. Composite indices of potential mediators are adjusted for each other and for age and sex. The thick horizontal line indicates the mean difference in retinal arteriolar \%-dilation (left) and skin \%-hyperemia (right) between individuals with type 2 diabetes and those with normal glucose metabolism, and thus corresponds with a mediation effect of 100\%. Abbreviations as in Supplemental Figure S3.2. 


\section{Supplemental Tables}

Table S3.1 General characteristics of the skin hyperemia study population according to glucose metabolism status

\begin{tabular}{|c|c|c|c|}
\hline Characteristic & $\begin{array}{c}\text { NGM } \\
\mathrm{n}=698\end{array}$ & $\begin{array}{c}\text { Prediabetes } \\
n=207\end{array}$ & $\begin{array}{c}\text { Type } 2 \text { diabetes } \\
\mathrm{n}=376\end{array}$ \\
\hline Age (years) & $58.7 \pm 8.1$ & $61.7 \pm 7.2$ & $62.6 \pm 7.6$ \\
\hline Women & 397 (56.9) & $88(42.5)$ & $119(31.6)$ \\
\hline Diabetes duration (years) ${ }^{*}$ & - & - & $6.0[3.0-11.0]$ \\
\hline Body mass index $\left(\mathrm{kg} / \mathrm{m}^{2}\right)$ & $25.5 \pm 3.6$ & $27.7 \pm 3.9$ & $29.2 \pm 4.5$ \\
\hline \multicolumn{4}{|l|}{ Waist circumference $(\mathrm{cm})$} \\
\hline - Men & $96.4 \pm 9.3$ & $101.9 \pm 9.9$ & $106.0 \pm 11.5$ \\
\hline - Women & $86.6 \pm 10.0$ & $92.8 \pm 12.3$ & $101.2 \pm 14.4$ \\
\hline History of cardiovascular disease & $92(13.6)$ & $27(13.5)$ & $91(25.9)$ \\
\hline Office SBP (mmHg) & $131.4 \pm 17.0$ & $138.5 \pm 16.5$ & $142.8 \pm 18.4$ \\
\hline Office DBP (mmHg) & $75.6 \pm 9.6$ & $79.0 \pm 9.5$ & $77.2 \pm 9.5$ \\
\hline Ambulatory 24-h SBP (mmHg) & $117.4 \pm 10.6$ & $121.6 \pm 12.3$ & $123.5 \pm 12.3$ \\
\hline Ambulatory 24-h DBP (mmHg) & $73.7 \pm 6.8$ & $75.0 \pm 7.2$ & $73.2 \pm 7.0$ \\
\hline Ambulatory 24-h PP (mmHg) & $43.7 \pm 7.1$ & $46.6 \pm 9.1$ & $50.4 \pm 9.9$ \\
\hline Ambulatory 24-h MAP (mmHg) & $88.2 \pm 7.6$ & $90.5 \pm 8.2$ & $89.9 \pm 7.8$ \\
\hline \multicolumn{4}{|l|}{ Smoking } \\
\hline - Never / former / current & $242 / 360 / 81$ & $60 / 123 / 19$ & $103 / 203 / 49$ \\
\hline - \% (never / former / current) & $35.4 / 52.7 / 11.9$ & $29.7 / 60.9 / 9.4$ & $29.0 / 57.2 / 13.8$ \\
\hline Fasting glucose (mmol/l) & $5.1 \pm 0.4$ & $5.9 \pm 0.6$ & $7.7 \pm 1.5$ \\
\hline 2-h postload glucose $(\mathrm{mmol} / \mathrm{l})^{+}$ & $5.4 \pm 1.1$ & $8.1 \pm 1.8$ & $14.4 \pm 3.9$ \\
\hline $\mathrm{HbA1c}(\%)$ & $5.5 \pm 0.3$ & $5.7 \pm 0.4$ & $6.8 \pm 0.9$ \\
\hline $\mathrm{HbA} 1 \mathrm{c}(\mathrm{mmol} / \mathrm{mol})$ & $36.6 \pm 3.6$ & $39.1 \pm 4.7$ & $50.7 \pm 10.1$ \\
\hline Skin auto-fluorescence (AU) & $2.4 \pm 0.5$ & $2.6 \pm 0.5$ & $2.7 \pm 0.6$ \\
\hline Total-to-HDL cholesterol ratio & $3.6 \pm 1.1$ & $4.0 \pm 1.2$ & $3.7 \pm 1.1$ \\
\hline Total cholesterol (mmol/l) & $5.6 \pm 1.1$ & $5.6 \pm 1.2$ & $4.4 \pm 1.1$ \\
\hline HDL cholesterol (mmol/l) & $1.7 \pm 0.5$ & $1.5 \pm 0.4$ & $1.3 \pm 0.4$ \\
\hline LDL cholesterol (mmol/l) & $3.4 \pm 1.0$ & $3.4 \pm 1.1$ & $2.4 \pm 0.9$ \\
\hline Triglycerides (mmol/l) & $1.2 \pm 0.6$ & $1.7 \pm 1.3$ & $1.7 \pm 0.9$ \\
\hline Antihypertensive medication use & $178(25.5)$ & $92(44.4)$ & $260(69.1)$ \\
\hline Lipid-modifying medication use & $145(20.8)$ & $68(32.9)$ & $282(75.0)$ \\
\hline \multicolumn{4}{|l|}{ Diabetes medication use } \\
\hline - Any type & - & - & $287(76.3)$ \\
\hline - Insulin & - & - & $68(18.1)$ \\
\hline - Oral glucose-lowering medication & - & - & $272(72.3)$ \\
\hline HOMA2-IR Insulin $(A U)$ & $1.3 \pm 0.7$ & $2.0 \pm 1.2$ & $2.3 \pm 1.4$ \\
\hline HOMA2-IR -peptide $(A U)$ & $1.3 \pm 0.5$ & $1.8 \pm 0.8$ & $2.1 \pm 0.9$ \\
\hline \multicolumn{4}{|l|}{ Markers of low-grade inflammation } \\
\hline - High-sensitive CRP (mg/l) & $1.1[0.6-2.3]$ & $1.7[0.9-3.7]$ & $1.4[0.7-3.2]$ \\
\hline - Serum amyloid A (mg/l) & $3.2[2.0-5.1]$ & $3.6[2.3-5.6]$ & $3.5[2.1-5.9]$ \\
\hline - Soluble ICAM-1 (ng/ml) & $339.3 \pm 77.4$ & $368.6 \pm 87.2$ & $382.2 \pm 118.2$ \\
\hline - Interleukin-6 (pg/ml) & $0.5[0.4-0.8]$ & $0.7[0.5-0.9]$ & $0.7[0.5-1.1]$ \\
\hline - Interleukin-8 (pg/ml) & $3.8[3.1-4.7]$ & $4.3[3.4-5.3]$ & $4.8[3.9-6.1]$ \\
\hline - Tumor necrosis factor- $\alpha$ (pg/ml) & $2.1[1.8-2.4]$ & $2.3[1.9-2.6]$ & $2.3[2.0-2.9]$ \\
\hline
\end{tabular}


Table S3.1 (continued)

\begin{tabular}{|c|c|c|c|}
\hline Characteristic & $\begin{array}{l}\mathrm{NGM} \\
\mathrm{n}=698\end{array}$ & $\begin{array}{c}\text { Prediabetes } \\
n=207\end{array}$ & $\begin{array}{c}\text { Type } 2 \text { diabetes } \\
n=376\end{array}$ \\
\hline \multicolumn{4}{|l|}{ Markers of arterial stiffness } \\
\hline - Carotid-femoral PWV (m/s) & $8.6 \pm 1.8$ & $9.4 \pm 2.3$ & $9.9 \pm 2.5$ \\
\hline - Carotid DC $\left(10^{3} / \mathrm{kPa}\right)$ & $14.6 \pm 5.1$ & $13.3 \pm 5.0$ & $12.9 \pm 4.7$ \\
\hline eGFR (ml/min $\left./ 1.73 \mathrm{~m}^{2}\right)$ & $89.4 \pm 13.1$ & $86.6 \pm 14.1$ & $86.1 \pm 17.1$ \\
\hline $\mathrm{eGFR}<60 \mathrm{ml} / \mathrm{min} / 1.73 \mathrm{~m}^{2}$ & $12(1.7)$ & $8(3.9)$ & $30(8.0)$ \\
\hline (Micro)albuminuria $^{\ddagger}$ & $25(3.6)$ & $14(6.8)$ & $62(16.6)$ \\
\hline Retinopathy & $1(0.2)$ & $0(0.0)$ & $15(4.2)$ \\
\hline Baseline skin blood flow (PU) & $10.8 \pm 6.4$ & $11.7 \pm 7.2$ & $11.0 \pm 5.7$ \\
\hline \multicolumn{4}{|l|}{ Skin hyperemic response (\%) } \\
\hline- Mean \pm SD & $1252.6 \pm 813.4$ & $1107.4 \pm 710.8$ & $941.7 \pm 701.1$ \\
\hline - Median [interquartile range] & 1104.0 [668.7-1656.9] & 1006.9 [604.9-1536.9] & 821.2 [479.0-1209.8] \\
\hline
\end{tabular}

Data are reported as mean $\pm \mathrm{SD}$, median [interquartile range], or number (percentage \%) as appropriate. NGM, normal glucose metabolism; SD, standard deviation; SBP, systolic blood pressure; DBP, diastolic blood pressure; PP, pulse pressure; MAP, mean arterial pressure; HbA1c, glycated hemoglobin A1c; AU, arbitrary units; HDL, high-density lipoprotein; LDL, low-density lipoprotein; HOMA2-IR, homeostasis model assessment of insulin resistance; CRP, C-reactive protein; ICAM, intercellular adhesion molecule; PWV, pulse wave velocity; DC, distensibility coefficient; eGFR, estimated glomerular filtration rate; PU, perfusion units. ${ }^{*}$ Available in 254 individuals with type 2 diabetes. ${ }^{\dagger}$ Available in 308 individuals with type 2 diabetes. ${ }^{\ddagger}$ (Micro)albuminuria was defined as a urinary albumin excretion of $>30 \mathrm{mg}$ per 24 hours.

Table S3.2 General characteristics of the skin hyperemia study population and individuals excluded from the analyses due to missing values

\begin{tabular}{|c|c|c|c|c|c|}
\hline Characteristic & $\begin{array}{l}\text { Skin hyperemia } \\
\text { study population } \\
(n=1281)\end{array}$ & $\begin{array}{l}\text { Missings in skin } \\
\text { hyperemia study } \\
\text { population* }\end{array}$ & $\begin{array}{l}\text { Excluded due to } \\
\text { missing values } \\
(n=2129)\end{array}$ & $\begin{array}{c}\text { Missings in population } \\
\text { excluded due to } \\
\text { missing values }^{+}\end{array}$ & P-value \\
\hline Age (years) & $60.3 \pm 8.0$ & 0 & $59.5 \pm 8.4$ & 0 & 0.906 \\
\hline Women & $604(47.2)$ & 0 & $1050(49.3)$ & 0 & 0.769 \\
\hline Glucose metabolism status & & 0 & & 0 & 0.019 \\
\hline - Normal glucose metabolism & $698(54.5)$ & & $1226(57.6)$ & & \\
\hline - Prediabetes & $207(16.2)$ & & $304(14.3)$ & & \\
\hline - Type 2 diabetes & $376(29.4)$ & & $599(28.1)$ & & \\
\hline Diabetes duration (years) & $6.0[3.0-11.0]$ & 122 & $7.0[3.0-13.0]$ & 189 & 0.155 \\
\hline Body mass index $\left(\mathrm{kg} / \mathrm{m}^{2}\right)$ & $27.0 \pm 4.2$ & 1 & $27.1 \pm 4.7$ & 2 & 0.001 \\
\hline Waist circumference $(\mathrm{cm})$ & & 0 & & 4 & \\
\hline - Men & $101.0 \pm 11.1$ & & $101.9 \pm 12.7$ & & 0.009 \\
\hline - Women & $90.4 \pm 12.7$ & & $89.9 \pm 13.2$ & & 0.007 \\
\hline History of cardiovascular disease & $210(17.1)$ & 53 & $342(16.5)$ & 52 & 0.013 \\
\hline Office SBP (mmHg) & $135.9 \pm 18.1$ & 1 & $134.5 \pm 18.3$ & 1 & 0.716 \\
\hline Office DBP (mmHg) & $76.6 \pm 9.6$ & 1 & $76.0 \pm 9.9$ & 1 & 0.111 \\
\hline Ambulatory 24-h SBP ( $\mathrm{mmHg}$ ) & $119.9 \pm 11.7$ & 0 & $118.4 \pm 11.9$ & 396 & 0.787 \\
\hline Ambulatory 24-h DBP (mmHg) & $73.7 \pm 6.9$ & 0 & $73.3 \pm 7.3$ & 396 & 0.514 \\
\hline Ambulatory 24-h PP (mmHg) & $46.1 \pm 8.8$ & 0 & $45.1 \pm 8.7$ & 396 & 0.865 \\
\hline Ambulatory 24-h MAP (mmHg) & $89.1 \pm 7.8$ & 0 & $88.4 \pm 8.1$ & 396 & 0.601 \\
\hline Smoking & & 41 & & 20 & 0.009 \\
\hline - Never / former / current & $405 / 686 / 149$ & & $755 / 1043 / 311$ & & \\
\hline - \% (never / former / current) & $32.7 / 55.3 / 12.0$ & & 25.8/49.5/14.7 & & \\
\hline
\end{tabular}


Table S3.2 (continued)

\begin{tabular}{|c|c|c|c|c|c|}
\hline Characteristic & $\begin{array}{c}\text { Skin hyperemia } \\
\text { study population } \\
(n=1281)\end{array}$ & $\begin{array}{l}\text { Missings in skin } \\
\text { hyperemia study } \\
\text { population* }\end{array}$ & $\begin{array}{c}\text { Excluded due to } \\
\text { missing values } \\
(n=2129)\end{array}$ & $\begin{array}{c}\text { Missings in population } \\
\text { excluded due to } \\
\text { missing values }\end{array}$ & P-value \\
\hline Fasting glucose (mmol/l) & $6.0 \pm 1.4$ & 0 & $6.1 \pm 1.8$ & 1 & $<0.001$ \\
\hline 2-h postload glucose $(\mathrm{mmol} / \mathrm{l})$ & $8.1 \pm 4.4$ & 68 & $7.7 \pm 4.1$ & 183 & 0.738 \\
\hline HbA1c (\%) & $5.9 \pm 0.8$ & 0 & $5.9 \pm 1.0$ & 13 & $<0.001$ \\
\hline $\mathrm{HbA} 1 \mathrm{c}(\mathrm{mmol} / \mathrm{mol})$ & $41.1 \pm 8.9$ & 0 & $41.1 \pm 10.5$ & 13 & $<0.001$ \\
\hline Skin auto-fluorescence (AU) & $2.5 \pm 0.5$ & 0 & $2.4 \pm 0.5$ & 200 & $<0.001$ \\
\hline Total-to-HDL cholesterol ratio & $3.7 \pm 1.1$ & 0 & $3.7 \pm 1.2$ & 4 & 0.002 \\
\hline Total cholesterol (mmol/l) & $5.3 \pm 1.2$ & 0 & $5.2 \pm 1.1$ & 4 & 0.019 \\
\hline HDL cholesterol (mmol/l) & $1.5 \pm 0.5$ & 0 & $1.5 \pm 0.5$ & 4 & $<0.001$ \\
\hline LDL cholesterol (mmol/l) & $3.1 \pm 1.1$ & 0 & $3.1 \pm 1.0$ & 4 & 0.607 \\
\hline Triglycerides (mmol/l) & $1.5 \pm 0.9$ & 0 & $1.4 \pm 0.8$ & 4 & 0.628 \\
\hline Antihypertensive medication use & $530(41.4)$ & 0 & $825(38.8)$ & 4 & 0.005 \\
\hline Lipid-modifying medication use & $495(38.6)$ & 0 & $725(34.1)$ & 4 & 0.142 \\
\hline \multicolumn{6}{|l|}{ Diabetes medication use } \\
\hline - Any type & $287(22.4)$ & 0 & $478(22.5)$ & 4 & $<0.001$ \\
\hline - Insulin & $68(5.3)$ & 0 & $148(7.0)$ & 4 & $<0.001$ \\
\hline - Oral glucose-lowering medication & $272(21.2)$ & 0 & $441(20.8)$ & 4 & 0.002 \\
\hline HOMA2-IR Insulin (AU) & $1.7 \pm 1.1$ & 0 & $1.7 \pm 1.2$ & 198 & 0.054 \\
\hline HOMA2-IR $\mathrm{R}_{\text {-peptide }}(\mathrm{AU})$ & $1.6 \pm 0.8$ & 0 & $1.6 \pm 1.0$ & 55 & 0.003 \\
\hline \multicolumn{6}{|l|}{ Markers of low-grade inflammation } \\
\hline - High-sensitive CRP (mg/l) & $1.2[0.6-2.7]$ & 0 & $1.3[0.6-2.9]$ & 38 & 0.152 \\
\hline - Serum amyloid A (mg/l) & $3.3[2.1-5.5]$ & 0 & $3.2[2.0-5.4]$ & 38 & 0.388 \\
\hline - Soluble ICAM-1 (ng/ml) & $356.6 \pm 94.7$ & 0 & $353.6 \pm 103.2$ & 38 & 0.981 \\
\hline - Interleukin-6 (pg/ml) & $0.6[0.4-0.9]$ & 0 & $0.6[0.4-0.9]$ & 39 & 0.446 \\
\hline - Interleukin-8 (pg/ml) & $4.1[3.3-5.2]$ & 0 & $4.1[3.3-5.4]$ & 38 & $<0.001$ \\
\hline - Tumor necrosis factor- $\alpha(\mathrm{pg} / \mathrm{ml})$ & $2.2[1.9-2.5]$ & 0 & $2.2[1.9-2.6]$ & 38 & 0.251 \\
\hline \multicolumn{6}{|l|}{ Markers of arterial stiffness } \\
\hline - Carotid-femoral PWV (m/s) & $9.1 \pm 2.2$ & 168 & $9.0 \pm 2.1$ & 391 & 0.168 \\
\hline - Carotid DC (103/kPa) & $13.9 \pm 5.0$ & 157 & $14.5 \pm 5.2$ & 359 & 0.164 \\
\hline $\operatorname{eGFR}\left(\mathrm{ml} / \mathrm{min} / 1.73 \mathrm{~m}^{2}\right)$ & $88.0 \pm 14.6$ & 5 & $88.2 \pm 15.0$ & 28 & 0.453 \\
\hline eGFR<60 ml/min $/ 1.73 \mathrm{~m}^{2}$ & $50(3.9)$ & 5 & $93(4.4)$ & 28 & 0.647 \\
\hline (Micro)albuminuria $^{\ddagger}$ & $101(7.9)$ & 7 & $190(9.1)$ & 35 & 0.067 \\
\hline Retinopathy & $16(1.4)$ & 118 & $24(1.4)$ & 469 & 0.127 \\
\hline Baseline skin blood flow (PU) & $11.0 \pm 6.3$ & 0 & $11.4 \pm 6.6^{5}$ & 1763 & 0.773 \\
\hline \multicolumn{6}{|l|}{ Skin hyperemic response (\%) } \\
\hline - Mean \pm SD & $1137.9 \pm 777.3$ & 0 & $1080.9 \pm 752.3^{\S}$ & 1763 & 0.214 \\
\hline - Median [interquartile range] & $\begin{array}{c}1012.2 \\
{[594.4-1516.0]}\end{array}$ & 0 & $\begin{array}{c}913.1 \\
{[529.4-1415.4]^{5}}\end{array}$ & 1763 & 0.214 \\
\hline
\end{tabular}

Data are reported as mean \pm SD, median [interquartile range], or number (percentage \%) as appropriate. $\mathrm{P}$-value indicates comparison between study population and individuals excluded due to missing values. SD, standard deviation; SBP, systolic blood pressure; DBP, diastolic blood pressure; PP, pulse pressure; MAP, mean arterial pressure; HbA1c, glycated hemoglobin A1c; AU, arbitrary units; HDL, high-density lipoprotein; LDL, low-density lipoprotein; HOMA2-IR, homeostasis model assessment of insulin resistance; CRP, C-reactive protein; ICAM, intercellular adhesion molecule; PWV, pulse wave velocity; DC, distensibility coefficient; eGFR, estimated glomerular filtration rate; PU, perfusion units. ${ }^{*}$ Total number of missings for a specific variable in the skin hyperemia study population, ${ }^{\dagger}$ Total number of missings for a specific variable in the population which was excluded, ${ }^{\ddagger}$ (Micro)albuminuria was defined as a urinary albumin excretion of $>30$ mg per 24 hours, ${ }^{\S} 366$ were excluded due to missing on potential mediator. 
Table S3.3 General characteristics of the retinal reactivity study population and individuals excluded from the analyses due to missing values

\begin{tabular}{|c|c|c|c|c|c|}
\hline Characteristic & $\begin{array}{c}\text { Retinal reactivity } \\
\text { study population } \\
\quad(n=1791)\end{array}$ & $\begin{array}{c}\text { Missings in retinal } \\
\text { reactivity study } \\
\text { population* }\end{array}$ & $\begin{array}{c}\text { Excluded due to } \\
\text { missing values } \\
(n=1619)\end{array}$ & $\begin{array}{c}\text { Missings in population } \\
\text { excluded due to } \\
\text { missing values }{ }^{+}\end{array}$ & P-value \\
\hline Age (years) & $59.8 \pm 8.2$ & 0 & $59.8 \pm 8.3$ & 0 & 0.906 \\
\hline Women & $873(48.7)$ & 0 & $781(48.2)$ & 0 & 0.769 \\
\hline Glucose metabolism status & & 0 & & 0 & 0.019 \\
\hline - Normal glucose metabolism & $1040(58.0)$ & & $884(54.6)$ & & \\
\hline - Prediabetes & $276(15.4)$ & & $235(14.5)$ & & \\
\hline - Type 2 diabetes & $475(26.5)$ & & $500(30.9)$ & & \\
\hline Diabetes duration (years) & $6.0[3.0-12.0]$ & 124 & $7.0[3.0-12.0]$ & 187 & 0.155 \\
\hline Body mass index $\left(\mathrm{kg} / \mathrm{m}^{2}\right)$ & $26.9 \pm 4.2$ & 2 & $27.4 \pm 4.9$ & 1 & 0.001 \\
\hline Waist circumference $(\mathrm{cm})$ & & 1 & & 3 & \\
\hline - Men & $100.9 \pm 11.2$ & & $102.4 \pm 13.1$ & & 0.009 \\
\hline - Women & $89.3 \pm 12.3$ & & $91.0 \pm 13.7$ & & 0.007 \\
\hline History of cardiovascular disease & $264(15.2)$ & 51 & $288(18.4)$ & 54 & 0.013 \\
\hline Office SBP (mmHg) & $134.9 \pm 17.8$ & 2 & $135.1 \pm 18.6$ & 0 & 0.716 \\
\hline Office DBP (mmHg) & $76.5 \pm 9.9$ & 2 & $75.9 \pm 9.7$ & 0 & 0.111 \\
\hline Ambulatory 24-h SBP (mmHg) & $119.1 \pm 11.6$ & 0 & $119.0 \pm 12.1$ & 396 & 0.787 \\
\hline Ambulatory 24-h DBP (mmHg) & $73.6 \pm 7.2$ & 0 & $73.4 \pm 7.1$ & 396 & 0.514 \\
\hline Ambulatory 24-h PP (mmHg) & $45.5 \pm 8.4$ & 0 & $45.6 \pm 9.1$ & 396 & 0.865 \\
\hline Ambulatory 24-h MAP (mmHg) & $88.7 \pm 8.0$ & 0 & $89.6 \pm 8.0$ & 396 & 0.601 \\
\hline Smoking & & 33 & & 28 & 0.009 \\
\hline - Never / former / current & $620 / 927 / 211$ & & $540 / 802 / 249$ & & \\
\hline - \% (never / former / current) & $35.3 / 52.7 / 12.0$ & & $33.4 / 50.4 / 15.7$ & & \\
\hline Fasting glucose (mmol/l) & $6.0 \pm 1.4$ & 0 & $6.2 \pm 1.9$ & 1 & $<0.001$ \\
\hline 2-h postload glucose $(\mathrm{mmol} / \mathrm{l})$ & $7.9 \pm 4.2$ & 86 & $7.9 \pm 4.3$ & 165 & 0.738 \\
\hline HbA1c (\%) & $5.8 \pm 0.8$ & 0 & $6.0 \pm 1.0$ & 13 & $<0.001$ \\
\hline $\mathrm{HbA1c}(\mathrm{mmol} / \mathrm{mol})$ & $40.1 \pm 8.8$ & 0 & $42.2 \pm 11.0$ & 13 & $<0.001$ \\
\hline Skin auto-fluorescence (AU) & $2.4 \pm 0.5$ & 0 & $2.5 \pm 0.6$ & 200 & $<0.001$ \\
\hline Total-to-HDL cholesterol ratio & $3.6 \pm 1.1$ & 0 & $3.7 \pm 1.2$ & 4 & 0.002 \\
\hline Total cholesterol (mmol/l) & $5.3 \pm 1.2$ & 0 & $5.2 \pm 1.1$ & 4 & 0.019 \\
\hline HDL cholesterol (mmol/l) & $1.6 \pm 0.5$ & 0 & $1.5 \pm 0.5$ & 4 & $<0.001$ \\
\hline LDL cholesterol (mmol/l) & $3.1 \pm 1.0$ & 0 & $3.1 \pm 1.0$ & 4 & 0.607 \\
\hline Triglycerides (mmol/l) & $1.4 \pm 0.8$ & 0 & $1.4 \pm 0.9$ & 4 & 0.628 \\
\hline Antihypertensive medication use & $672(37.5)$ & 0 & $683(42.3)$ & 4 & 0.005 \\
\hline Lipid-modifying medication use & $621(34.7)$ & 0 & $599(37.1)$ & 4 & 0.142 \\
\hline \multicolumn{6}{|l|}{ Diabetes medication use } \\
\hline - Any type & $359(20.0)$ & 0 & $406(25.1)$ & 4 & $<0.001$ \\
\hline - Insulin & $86(4.8)$ & 0 & $130(8.0)$ & 4 & $<0.001$ \\
\hline - Oral glucose-lowering medication & 338 (18.9) & 0 & $375(23.2)$ & 4 & 0.002 \\
\hline HOMA2-IR Insulin $(A U)$ & $1.7 \pm 1.1$ & 0 & $1.8 \pm 1.2$ & 198 & 0.054 \\
\hline HOMA2-IR & $1.6 \pm 0.8$ & 0 & $1.7 \pm 1.0$ & 55 & 0.003 \\
\hline \multicolumn{6}{|l|}{ Markers of low-grade inflammation } \\
\hline - High-sensitive CRP (mg/l) & $1.2[0.6-2.7]$ & 0 & $1.3[0.6-2.9]$ & 38 & 0.152 \\
\hline - Serum amyloid A (mg/l) & $3.2[2.0-5.4]$ & 0 & $3.3[2.1-5.6]$ & 38 & 0.388 \\
\hline - Soluble ICAM-1 (ng/ml) & $354.8 \pm 96.5$ & 0 & $354.7 \pm 103.9$ & 38 & 0.981 \\
\hline - Interleukin-6 (pg/ml) & $0.6[0.4-0.9]$ & 0 & $0.6[0.4-0.9]$ & 39 & 0.446 \\
\hline - Interleukin-8 (pg/ml) & $4.1[3.2-5.2]$ & 0 & $4.3[3.4-5.6]$ & 38 & $<0.001$ \\
\hline - Tumor necrosis factor- $\alpha(\mathrm{pg} / \mathrm{ml})$ & $2.2[1.9-2.6]$ & 0 & $2.2[1.9-2.6]$ & 38 & 0.251 \\
\hline
\end{tabular}


Table S3.3 (continued)

\begin{tabular}{|c|c|c|c|c|c|}
\hline Characteristic & $\begin{array}{c}\text { Retinal reactivity } \\
\text { study population } \\
(n=1791)\end{array}$ & $\begin{array}{l}\text { Missings in retinal } \\
\text { reactivity study } \\
\text { population* }\end{array}$ & $\begin{array}{c}\text { Excluded due to } \\
\text { missing values } \\
(n=1619)\end{array}$ & $\begin{array}{c}\text { Missings in population } \\
\text { excluded due to missing } \\
\text { values }^{+}\end{array}$ & P-value \\
\hline \multicolumn{6}{|l|}{ Markers of arterial stiffness } \\
\hline - Carotid-femoral PWV (m/s) & $9.0 \pm 2.0$ & 304 & $9.1 \pm 2.3$ & 255 & 0.168 \\
\hline - Carotid DC $\left(10^{3} / \mathrm{kPa}\right)$ & $14.4 \pm 5.1$ & 284 & $14.2 \pm 5.1$ & 232 & 0.164 \\
\hline eGFR (ml/min $\left./ 1.73 \mathrm{~m}^{2}\right)$ & $88.3 \pm 14.5$ & 4 & $87.9 \pm 15.2$ & 29 & 0.453 \\
\hline eGFR<60 ml $/ \mathrm{min} / 1.73 \mathrm{~m}^{2}$ & $73(4.1)$ & 4 & $70(4.4)$ & 29 & 0.647 \\
\hline (Micro)albuminuria $^{\ddagger}$ & $139(7.8)$ & 10 & $152(9.6)$ & 32 & 0.067 \\
\hline Retinopathy & $20(1.1)$ & 51 & $20(1.8)$ & 536 & 0.127 \\
\hline Baseline arteriolar diameter (MU) & $115.4 \pm 15.5$ & 0 & $115.7 \pm 16.0^{\S}$ & 1149 & 0.773 \\
\hline \multicolumn{6}{|l|}{ Arteriolar average dilation (\%) } \\
\hline- Mean \pm SD & $3.1 \pm 2.8$ & 0 & $2.9 \pm 2.7^{\S}$ & 1149 & 0.214 \\
\hline - Median [interquartile range] & $2.6[0.8-5.0]$ & 0 & $2.5[0.8-4.7]^{\S}$ & 1149 & 0.214 \\
\hline
\end{tabular}

Data are reported as mean \pm SD, median [interquartile range], or number (percentage \%) as appropriate. $\mathrm{P}$-value indicates comparison between study population and individuals excluded due to missing values. SD, standard deviation; SBP, systolic blood pressure; DBP, diastolic blood pressure; PP, pulse pressure; MAP, mean arterial pressure; HbA1c, glycated hemoglobin A1c; AU, arbitrary units; HDL, high-density lipoprotein; LDL, low-density lipoprotein; HOMA2-IR, homeostasis model assessment of insulin resistance; CRP, C-reactive protein; ICAM, intercellular adhesion molecule; PWV, pulse wave velocity; DC, distensibility coefficient; eGFR, estimated glomerular filtration rate; MU, measurement units. ${ }^{*}$ Total number of missings for a specific variable in the retinal reactivity study population, ${ }^{\dagger}$ Total number of missings for a specific variable in the population which was excluded, ${ }^{\ddagger}$ (Micro)albuminuria was defined as a urinary albumin excretion of $>30 \mathrm{mg}$ per 24 hours, ${ }^{\S} 470$ were excluded due to missing on potential mediator. 
Table S3.4 Age- and sex-adjusted mediating effect of markers of hyperglycemia, insulin resistance, blood pressure, arterial stiffness, lipid profile, and low-grade inflammation on type 2 diabetesassociated impaired retinal arteriolar \%-dilation and skin \%-hyperemia

\begin{tabular}{|c|c|c|}
\hline & $\begin{array}{l}\text { Retinal arteriolar \%-dilation } \\
\qquad(\mathrm{n}=1791)\end{array}$ & $\begin{array}{c}\text { Skin \%-hyperemia } \\
(n=1281)\end{array}$ \\
\hline \multicolumn{3}{|l|}{ Hyperglycemia } \\
\hline Fasting glucose $(\mathrm{mmol} / \mathrm{l})$ & $-0.42(-0.73 ;-0.11)^{*}$ & $-75(-159 ; 11)$ \\
\hline 2-h postload glucose $(\mathrm{mmol} / \mathrm{l})^{\dagger}$ & $0.19(-0.35 ; 0.74)$ & $-94(-264 ; 97)$ \\
\hline HbA1c (\%) & $-0.41(-0.67 ;-0.12)^{*}$ & $-97(-177 ;-19)^{*}$ \\
\hline Skin auto-fluorescence (AU) & $-0.04(-0.10 ; 0.02)$ & $-10(-32 ; 11)$ \\
\hline \multicolumn{3}{|l|}{ Insulin resistance } \\
\hline HOMA2-IR Insulin (AU) & $-0.00(-0.14 ; 0.15)$ & $-24(-61 ; 11)$ \\
\hline HOMA2-IR c-peptide $(A U)$ & $-0.00(-0.15 ; 0.15)$ & $-15(-54 ; 31)$ \\
\hline \multicolumn{3}{|l|}{ Blood pressure $^{\ddagger}$} \\
\hline Ambulatory 24-h SBP (mmHg) & $0.01(-0.00 ; 0.05)$ & $-9(-23 ; 1)$ \\
\hline Ambulatory 24-h DBP (mmHg) & $-0.02(-0.06 ;-0.00)^{*}$ & $-1(-10 ; 5)$ \\
\hline Antihypertensive medication use & $-0.13(-0.26 ;-0.01)^{*}$ & $-11(-46 ; 21)$ \\
\hline \multicolumn{3}{|l|}{ Markers of arterial stiffness ${ }^{5}$} \\
\hline Carotid-femoral PWV (m/s) & $0.01(-0.06 ; 0.09)$ & $11(-11 ; 38)$ \\
\hline Carotid DC $(*-1)\left(10^{3} / \mathrm{kPa}\right)^{1 !}$ & $-0.01(-0.05 ; 0.01)$ & $8(-2 ; 25)$ \\
\hline \multicolumn{3}{|l|}{ Lipid profile $^{\ddagger}$} \\
\hline Total cholesterol (mmol/l) & $-0.06(-0.14 ; 0.00)$ & $-13(-39 ; 9)$ \\
\hline HDL cholesterol $(*-1)(\mathrm{mmol} / \mathrm{l})^{\prime \prime}$ & $0.00(-0.11 ; 0.11)$ & $2(-30 ; 35)$ \\
\hline Triglycerides (mmol/l) & $-0.04(-0.11 ; 0.04)$ & $-14(-35 ; 2)$ \\
\hline Lipid-modifying medication use & $-0.13(-0.30 ; 0.03)$ & $-14(-60 ; 35)$ \\
\hline \multicolumn{3}{|l|}{ Markers of low-grade inflammation } \\
\hline (log) High-sensitive CRP (mg/l) & $-0.00(-0.06 ; 0.05)$ & $-3(-17 ; 10)$ \\
\hline (log) Serum amyloid A (mg/l) & $-0.03(-0.08 ; 0.01)$ & $6(-5 ; 21)$ \\
\hline Soluble ICAM-1 (ng/ml) & $-0.01(-0.07 ; 0.06)$ & $-17(-38 ; 1)$ \\
\hline (log) Interleukin-6 (pg/ml) & $-0.06(-0.12 ;-0.01)^{*}$ & $-0(-32 ; 29)$ \\
\hline (log) Interleukin-8 (pg/ml) & $0.02(-0.05 ; 0.10)$ & $3(-12 ; 19)$ \\
\hline (log) Tumor necrosis factor- $\alpha(\mathrm{pg} / \mathrm{ml})$ & $-0.03(-0.09 ; 0.01)$ & $-9(-26 ; 6)$ \\
\hline
\end{tabular}

Mediating effects are presented as the indirect effects $(95 \% \mathrm{Cl})$ of type 2 diabetes on retinal arteriolar dilation and skin hyperemia through the potential mediators in percentage microvascular function. All analyses are adjusted for age and sex. ${ }^{*} \mathrm{P}<0.05 .{ }^{\dagger}$ Available in $\mathrm{n}=1705$ for the retinal population and $\mathrm{n}=1213$ for the skin population. ${ }^{\ddagger}$ Mediating effects of markers of blood pressure and lipid profile are additionally adjusted for the use of antihypertensive or lipid-modifying medication, respectively. ${ }^{\S}$ Available in $n=1453$ for the retinal population and $n=1090$ for the skin population. "The variables HDL-cholesterol and carotid DC were multiplied by -1 , as a lower HDL-cholesterol represents dyslipidemia, and a lower carotid DC represents arterial stiffness. HbA1c, glycated hemoglobin A1c; AU, arbitrary units; HOMA2-IR, homeostatic model assessment of insulin resistance; SBP, systolic blood pressure; DBP, diastolic blood pressure; PWV, pulse wave velocity; DC, distensibility coefficient; HDL, high-density lipoprotein; CRP, C-reactive protein; ICAM, intercellular adhesion molecule. 


\section{References}

1. Schram MT, Sep SJ, van der Kallen CJ, Dagnelie PC, Koster A, Schaper NC, Henry RM and Stehouwer CD. The Maastricht Study: an extensive phenotyping study on determinants of type 2 diabetes, its complications and its comorbidities. Eur J Epidemiol. 2014;29:439-51.

2. Sörensen BM, Houben AJ, Berendschot TT, Schouten JS, Kroon AA, van der Kallen CJ, Henry RM, Koster A, Sep SJ, Dagnelie PC, Schaper NC, Schram MT and Stehouwer CD. Prediabetes and Type 2 Diabetes Are Associated With Generalized Microvascular Dysfunction: The Maastricht Study. Circulation. 2016;134:1339-52.

3. Nagel E, Vilser W, Fink A and Riemer T. Variance of retinal vessel diameter response to flicker light. A methodical clinical study. Ophthalmologe. 2006;103:114-9.

4. Muris DM, Houben AJ, Kroon AA, Henry RM, van der Kallen CJ, Sep SJ, Koster A, Dagnelie PC, Schram MT and Stehouwer CD. Age, waist circumference, and blood pressure are associated with skin microvascular flow motion: the Maastricht Study. J Hypertens. 2014;32:2439-49.

5. Spauwen PJ, van Eupen MG, Kohler S, Stehouwer CD, Verhey FR, van der Kallen CJ, Sep SJ, Koster A, Schaper NC, Dagnelie PC, Schalkwijk CG, Schram MT and van Boxtel MP. Associations of advanced glycation end-products with cognitive functions in individuals with and without type 2 diabetes: the maastricht study. J Clin Endocrinol Metab. 2015;100:951-60.

6. Wallace TM, Levy JC and Matthews DR. Use and abuse of HOMA modeling. Diabetes Care. 2004;27: 1487-95.

7. Spauwen PJ, van Boxtel MP, Verhey FR, Kohler S, Sep SJ, Koster A, Dagnelie PC, Henry RM, Schaper NC, van der Kallen CJ, Schram MT, Kroon AA and Stehouwer CD. Both Low and High 24-Hour Diastolic Blood Pressure Are Associated With Worse Cognitive Performance in Type 2 Diabetes: The Maastricht Study. Diabetes Care. 2015;38:1473-80.

8. O'Brien E, Coats A, Owens P, Petrie J, Padfield PL, Littler WA, de Swiet $M$ and Mee F. Use and interpretation of ambulatory blood pressure monitoring: recommendations of the British hypertension society. BMJ. 2000;320:1128-34.

9. Van Bortel LM, Laurent S, Boutouyrie P, Chowienczyk P, Cruickshank JK, De Backer T, Filipovsky J, Huybrechts S, Mattace-Raso FU, Protogerou AD, Schillaci G, Segers P, Vermeersch S, Weber T, Artery S, European Society of Hypertension Working Group on Vascular S, Function and European Network for Noninvasive Investigation of Large A. Expert consensus document on the measurement of aortic stiffness in daily practice using carotid-femoral pulse wave velocity. J Hypertens. 2012;30:445-8.

10. Geijselaers SL, Sep SJ, Schram MT, van Boxtel MP, van Sloten TT, Henry RM, Reesink KD, Kroon AA, Koster A, Schaper NC, Dagnelie PC, van der Kallen CJ, Biessels GJ and Stehouwer CD. Carotid stiffness is associated with impairment of cognitive performance in individuals with and without type 2 diabetes. The Maastricht Study. Atherosclerosis. 2016;253:186-93.

11. van Dooren FE, Schram MT, Schalkwijk CG, Stehouwer CD, Henry RM, Dagnelie PC, Schaper NC, van der Kallen CJ, Koster A, Sep SJ, Denollet J, Verhey FR and Pouwer F. Associations of low grade inflammation and endothelial dysfunction with depression - The Maastricht Study. Brain Behav Immun. 2016;56:390-6.

12. van Bussel BC, Ferreira I, van de Waarenburg MP, van Greevenbroek MM, van der Kallen CJ, Henry RM, Feskens EJ, Stehouwer CD and Schalkwijk CG. Multiple inflammatory biomarker detection in a prospective cohort study: a cross-validation between well-established single-biomarker techniques and an electrochemiluminescense-based multi-array platform. PLoS One. 2013;8:e58576.

13. Martens RJ, Henry RM, Houben AJ, van der Kallen CJ, Kroon AA, Schalkwijk CG, Schram MT, Sep SJ, Schaper NC, Dagnelie PC, Muris DM, Gronenschild EH, van der Sande FM, Leunissen KM, Kooman JP and Stehouwer CD. Capillary Rarefaction Associates with Albuminuria: The Maastricht Study. J Am Soc Nephrol. 2016;27:3748-57.

14. Inker LA, Schmid CH, Tighiouart H, Eckfeldt JH, Feldman HI, Greene T, Kusek JW, Manzi J, Van Lente F, Zhang YL, Coresh J, Levey AS and Investigators C-E. Estimating glomerular filtration rate from serum creatinine and cystatin C. N Engl J Med. 2012;367:20-9. 


\section{Part II}

(Modifiable) cardiovascular risk factors as determinants of microvascular function 



\section{Chapter 4}

Cardiovascular risk factors as determinants of retinal and skin microvascular function: The Maastricht Study

Ben M. Sörensen, Alfons J.H.M. Houben, Tos T.J.M. Berendschot, Jan S.A.G. Schouten, Abraham A. Kroon, Carla J.H. van der Kallen, Ronald M.A. Henry, Annemarie Koster, Pieter C. Dagnelie, Nicolaas C. Schaper, Miranda T. Schram, Coen D.A. Stehouwer 


\section{Abstract}

\section{Objective}

Microvascular dysfunction is an important underlying mechanism of microvascular diseases. Determinants (age, sex, hypertension, dyslipidemia, hyperglycemia, obesity, and smoking) of macrovascular diseases affect large-artery endothelial function. These risk factors also associate with microvascular diseases. We hypothesized that they are also determinants of microvascular (endothelial) function.

\section{Methods}

In The Maastricht Study, a type 2 diabetes-enriched population-based cohort study ( $n=1991,51 \%$ men, aged $59.7 \pm 8.2$ years), we determined microvascular function as flicker light-induced retinal arteriolar \%-dilation and heat-induced skin \%-hyperemia. Multiple linear regression analyses were used to assess the associations of cardiovascular risk factors (age, sex, waist circumference, totalto-high-density lipoprotein (HDL) cholesterol ratio, fasting plasma glucose (FPG), 24-h systolic blood pressure, and cigarette smoking) with retinal and skin microvascular function.

\section{Results}

In multivariate analyses, age and FPG were inversely associated with retinal and skin microvascular function (regression coefficients per standard deviation (SD) were $-0.11 \mathrm{SD}(95 \% \mathrm{Cl}$ : $-0.15 ;-0.06)$ and -0.12 SD $(-0.17 ;-0.07)$ for retinal arteriolar \%-dilation and -0.10SD (-0.16; -0.05) and -0.11SD $(-0.17 ;-0.06)$ for skin \%-hyperemia, respectively. Men and current smokers had $-0.43 S D$ $(-0.58 ;-0.27)$ and -0.32 SD $(-0.49 ;-0.15)$ lower skin \%-hyperemia, respectively. 24 -h systolic blood pressure, waist circumference, and total-to-HDL cholesterol ratio were not statistically significantly associated with these microvascular functions.

\section{Conclusions}

Associations between cardiovascular risk factors and retinal and skin microvascular function show a pattern that is partly similar to the associations between cardiovascular risk factors and macrovascular function. Impairment of microvascular function may constitute a pathway through which an adverse cardiovascular risk factor pattern may increase risk of diseases of (partly) microvascular origin. 


\section{Introduction}

Microvascular dysfunction is an important underlying mechanism in common diseases of (partly) microvascular origin such as heart failure ${ }^{1}$, (lacunar) stroke ${ }^{2}$, depression ${ }^{3}$, cognitive decline ${ }^{4}$, retinopathy ${ }^{5}$, chronic kidney disease ${ }^{6}$, and neuropathy ${ }^{5}$.

However, determinants of microvascular dysfunction in the general population are mostly unknown. Although several studies ${ }^{7-13}$ have investigated potential determinants of microvascular function, these studies were conducted in small numbers of highly selected individuals ${ }^{7-13}$ and were insufficiently adjusted $^{7-13}$ for potential confounders, which limits translation to the general population.

In the general population, age, sex, hypertension, dyslipidemia, hyperglycemia, obesity, and smoking are major determinants of macrovascular diseases (e.g. stroke, myocardial infarction, and peripheral artery disease) ${ }^{14}$, and have been shown to act through inducing large-artery endothelial dysfunction ${ }^{15,16}$. However, this does not necessarily imply that microvascular endothelial function is affected similarly, as endothelial cells are known to be heterogeneous, depending on their localization ${ }^{17}$. Nevertheless, many of these risk factors are also associated with microvascular diseases ${ }^{18-21}$. Thus, we hypothesized that these risk factors may also be determinants of microvascular (endothelial) function.

Therefore, this study aimed to determine, in a population-based setting, whether cardiovascular risk factors were associated with, and thus potential determinants of, retinal and skin microvascular endothelial function. We chose retina and skin because these are unique sites enabling direct and reproducible ${ }^{22,23}$ assessment of microvascular function, as measured by flicker light-induced retinal arteriolar dilation and heat-induced skin hyperemia.

\section{Methods}

\section{Study population and design}

We used data from The Maastricht Study, an observational prospective populationbased cohort study. The rationale and methodology have been described previously ${ }^{24}$. In brief, the study focuses on the etiology, pathophysiology, complications, and comorbidities of type 2 diabetes (T2D) and is characterized by an extensive phenotyping approach. Eligible for participation were all individuals aged between 40 and 75 years and living in the southern part of the Netherlands. Participants were recruited through mass media campaigns and from the municipal registries and the regional Diabetes Patient Registry via mailings. Recruitment was stratified according to known T2D status, 
with an oversampling of individuals with T2D, for reasons of efficiency. The present report includes cross-sectional data from the first 3451 participants, who completed the baseline survey between November 2010 and September 2013. The examinations of each participant were performed within a time window of three months. The study has been approved by the institutional medical ethical committee (NL31329.068.10) and the Minister of Health, Welfare and Sports of the Netherlands (Permit 131088-105234-PG). All participants gave written informed consent.

\section{Assessment of microvascular function}

All participants were asked to refrain from smoking and drinking caffeine-containing beverages three hours before the measurement. A light meal (breakfast and (or) lunch), low in fat content, was allowed if taken at least 90 minutes prior to the start of the measurements.

Retinal arteriolar vasodilation to flicker light exposure was measured by the Dynamic Vessel Analyzer (Imedos, Jena, Germany). Briefly, a baseline recording of 50 seconds was followed by 40 -second flicker light exposure followed by a 60 -second recovery period. Baseline diameter was calculated as the average diameter size of the 20-50 seconds recording and was expressed in measurement units (MU). Percentage dilation over baseline was based on the average dilation achieved at time points 10 and 40 seconds during the flicker stimulation period.

Heat-induced skin hyperemia was measured by laser-Doppler flowmetry (Perimed, Järfälla, Sweden). Briefly, skin blood flow, expressed in arbitrary perfusion units (PU), was recorded unheated for 2 minutes to serve as a baseline. After 2 minutes, the temperature of the laser-Doppler probe was rapidly and locally increased to $44^{\circ} \mathrm{C}$, and was kept constant until the end of the registration. The heat-induced skin hyperemic response was expressed as the percentage increase in average PU during the 23 minutes heating phase over the 2 minutes average baseline PU. Both measurements have extensively been described previously ${ }^{25}$; more details are provided in the Supplemental Methods.

\section{Definition of cardiovascular risk factors}

Cardiovascular risk factors considered were age, sex, waist circumference, fasting plasma glucose (FPG), total-to-high-density lipoprotein (HDL) cholesterol ratio, 24-h systolic blood pressure, and smoking status. We also considered lipid-modifying and antihypertensive medication. 


\section{Measurement of cardiovascular risk factors}

We determined body mass index, waist circumference, glucose levels, glycated hemoglobin A1c (HbA1c), 24-h ambulatory systolic and diastolic blood pressure, total and HDL cholesterol, and triglycerides as described previously ${ }^{24}$ and detailed in the Supplemental Material. Smoking status (never, former, current) and pack-years of smoking were assessed by web-based questionnaire ${ }^{24}$. Glucose metabolism status was defined according to the World Health Organization 2006 criteria, based on a standardized 2-h 75 gram oral glucose tolerance test (Supplemental Material).

\section{Measurement of covariates}

The use of lipid-modifying and antihypertensive medication was assessed during a medication interview where generic name, dose, and frequency were registered ${ }^{24}$. The assessment of history of cardiovascular disease, 24-h urinary albumin excretion, estimated glomerular filtration rate (eGFR), and the presence of retinopathy have been described previously ${ }^{24}$ (cf. the Supplemental Material).

\section{Statistical analysis}

All analyses were performed with Statistical Package for Social Sciences version 23.0 (IBM SPSS, Armonk, USA). Variables with a skewed distribution (diabetes duration and pack-years of smoking) were log10 transformed. Standardized multiple linear regression analyses were used to evaluate the associations of cardiovascular risk factors (age, sex, waist circumference, FPG, total-to-HDL cholesterol ratio, 24-h systolic blood pressure, and smoking status) with both retinal and skin microvascular function. To compare regression coefficients between cardiovascular risk factors, continuous measures of these risk factors were standardized into z-scores before analyses. Associations of cardiovascular risk factors were adjusted for each other and for additional covariates (the use of antihypertensive and lipid-modifying medication, history of cardiovascular disease, 24-h urinary albumin excretion, eGFR, and the presence of retinopathy). In additional analyses, FPG was substituted by HbA1c, 2-h postload, or by T2D (yes/no). Waist circumference was substituted by body mass index, and 24-h systolic blood pressure by 24-h diastolic blood pressure, 24-h mean arterial pressure, or 24-h pulse pressure.

Data were expressed as standardized regression coefficients and their 95\% confidence intervals $(95 \% \mathrm{Cl})$. A P-value $<0.05$ was considered statistically significant. The study, by design, oversampled individuals with T2D; we therefore investigated potential interactions between cardiovascular risk factors and T2D by adding interaction terms (the product of a cardiovascular risk factor and T2D) to the regression models. Similarly, 
interactions between the cardiovascular risk factors and sex were investigated (the product of a cardiovascular risk factor and sex). A $P_{\text {interaction }}<0.10$ was considered statistically significant. A $P_{\text {interaction }} \geq 0.10$ indicates that the association between a cardiovascular risk factor and retinal or skin microvascular function does not statistically significantly differ between individuals without and with $\mathrm{T} 2 \mathrm{D}$, or between women and men, respectively ${ }^{26}$. A non-significant $P_{\text {interaction }}$ between a cardiovascular risk factor and T2D therefore indicates that the association between that cardiovascular risk factor and retinal or skin microvascular function was not driven by the oversampling of individuals with T2D. This implies that the association observed in this T2D-enriched population can be considered valid for a non-oversampled population, i.e. the general population ${ }^{26}$. Collinearity diagnostics (i.e. tolerance $<0.10$ and/or variance inflation factor $>10$ ) were used to detect multicollinearity between the cardiovascular risk factors and covariates.

\section{Results}

\section{Study population}

From the initial 3451 participants, retinal arteriolar reactivity data were available in 2290. The reasons for missing data were logistical $(n=891)$, insufficient measurement quality ( $n=209)$, or contraindications $(n=61)$. Data on cardiovascular risk factors were missing in 299 participants, particularly on 24-h blood pressure $(n=260)$, mainly due to device availability. The population in which retinal arteriolar reactivity data were available thus consisted of 1991 participants. Heat-induced skin hyperemia data were available in 1676 of the 3451 participants. The reasons for missing data were logistical $(n=1662)$ or insufficient measurement quality $(n=113)$. Cardiovascular risk factors were missing in 249 participants, mainly due to missing 24-h blood pressure values $(n=201)$. The population in which heat-induced skin hyperemia data were available thus consisted of 1427 participants (Figure 4.1 shows the flow chart).

Table 4.1 shows general characteristics of the retinal arteriolar reactivity study population stratified into tertiles of retinal arteriolar \%-dilation. This study population had a mean \pm standard deviation (SD) age of $59.7 \pm 8.2$ years, $48.8 \%$ were women, $12.1 \%$ were current smokers, and $27.4 \%$ had T2D. In addition, when compared to individuals in the lowest tertile of retinal arteriolar \%-dilation, those in the middle and highest tertiles were on average younger and had lower fasting and 2-h postload glucose levels (Table 4.1). The skin study population overlapped for $73 \%$ with the retinal study population, and was comparable with regard to age, sex, and cardiometabolic risk profile (Table 4.1). 
Cardiovascular risk factors and microvascular function

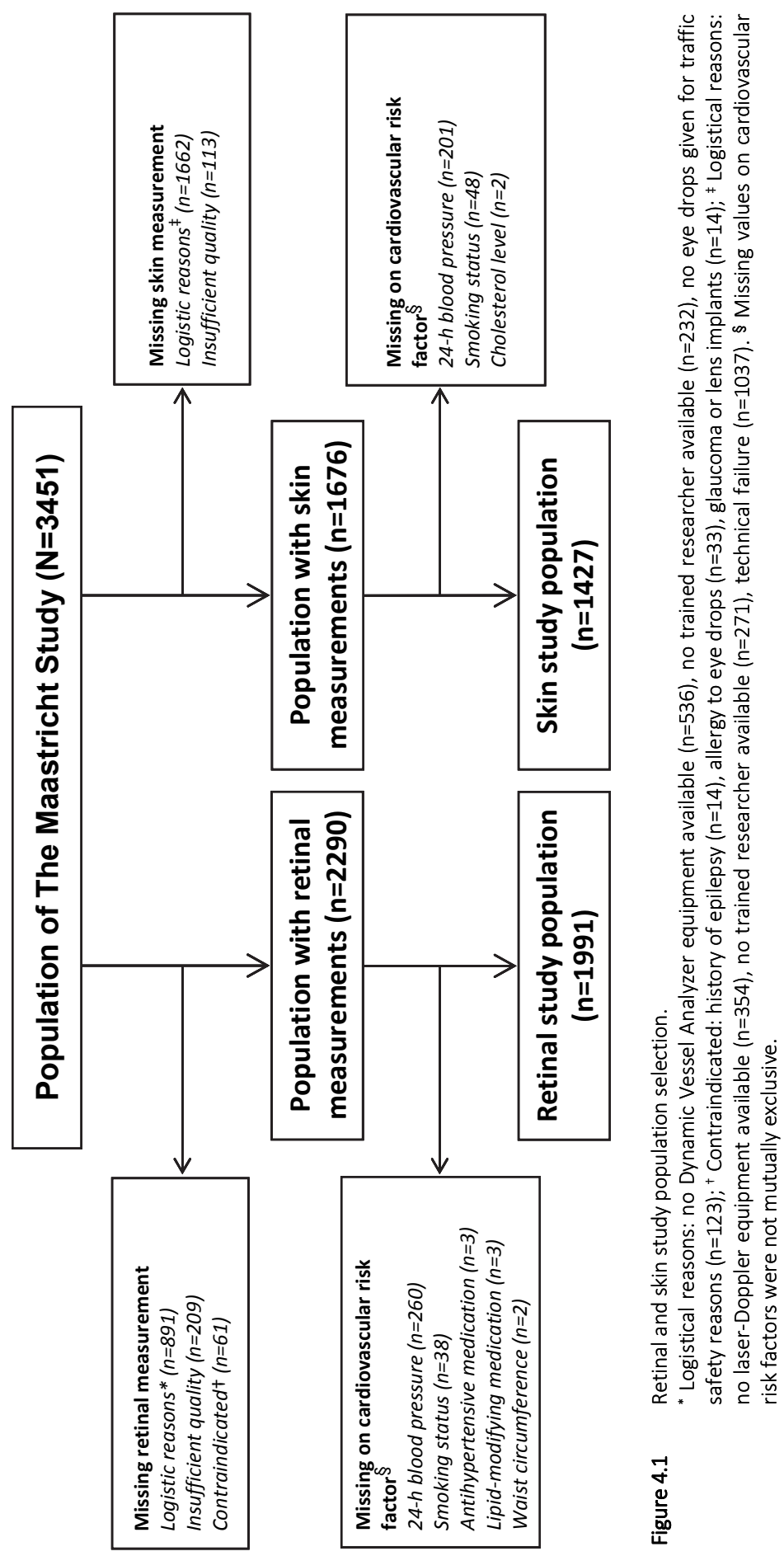




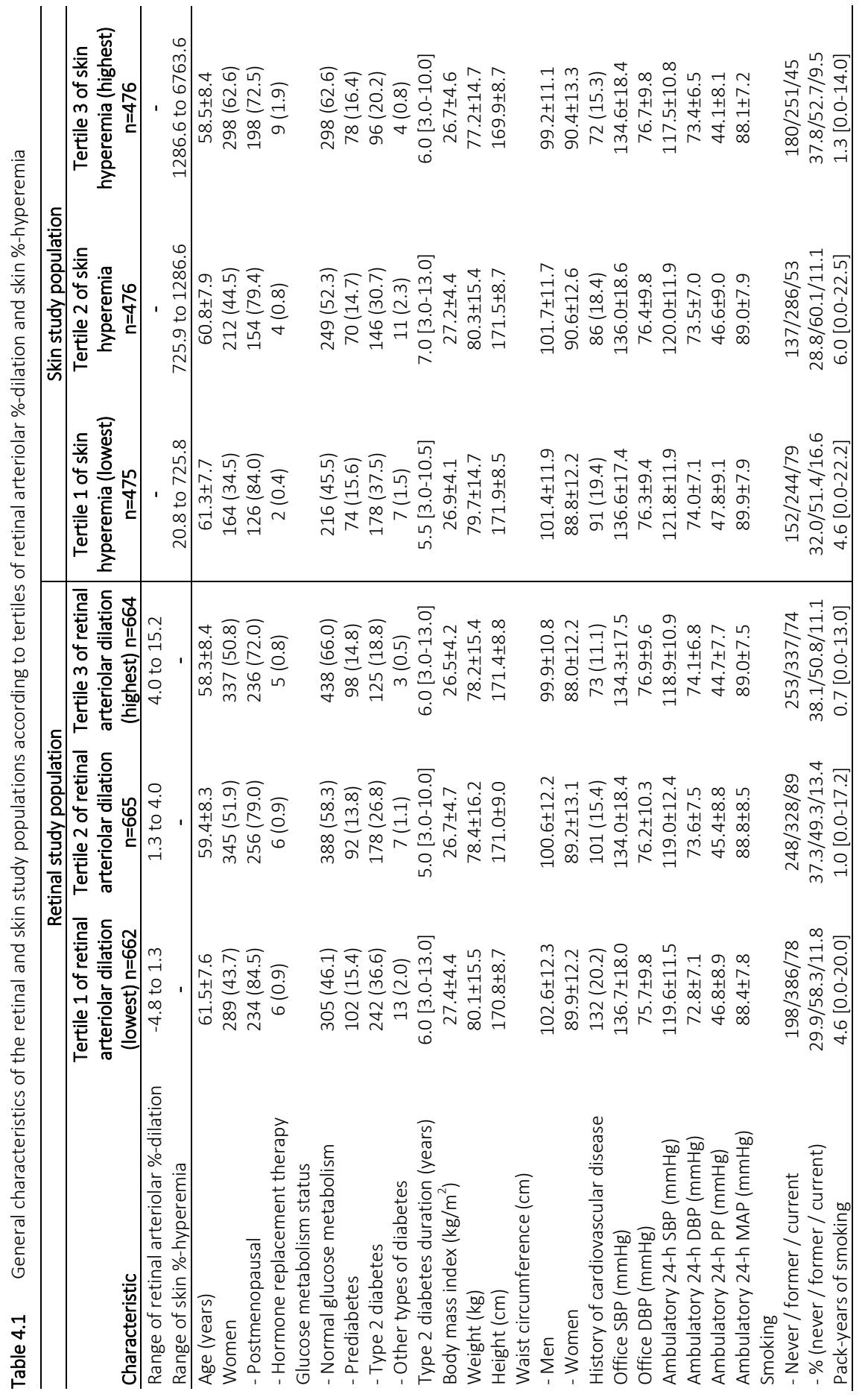




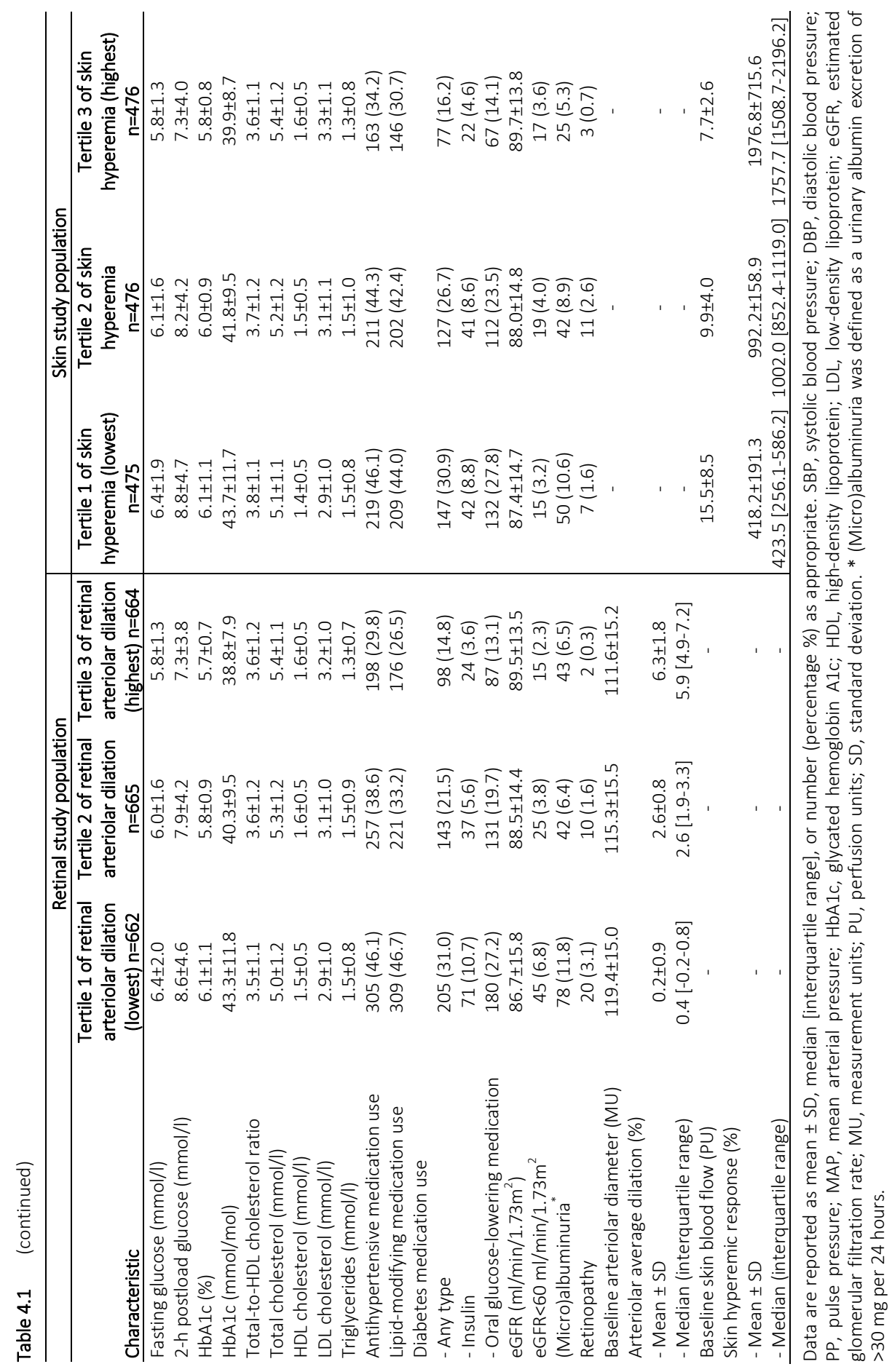


Individuals with missing data on retinal or skin reactivity measurements or measurements of cardiovascular risk factors were generally comparable to individuals included in the study populations with regard to age, sex, and cardiometabolic risk profile (Supplemental Tables S4.1 and S4.2).

\section{Age, sex and retinal arteriolar dilation and skin hyperemia}

Age was inversely associated with retinal arteriolar \%-dilation and skin \%-hyperemia; per SD higher age (8.2 years), retinal arteriolar \%-dilation was $-0.11 \mathrm{SD}(95 \% \mathrm{Cl}:-0.15 ;-0.06$, $\mathrm{P}<0.001)$ lower, and skin \%-hyperemia was $-0.10 \mathrm{SD}(-0.16 ;-0.05, \mathrm{P}<0.001)$ lower. Sex was not associated with retinal arteriolar \%-dilation, whereas men had a $-0.43 S D$ (-0.58; -0.27, $\mathrm{P}<0.001)$ lower skin \%-hyperemic response as compared to women (Figure 4.2). Moreover, the inverse association between age and skin \%-hyperemia was stronger in men $(-0.21 S D(-0.29 ;-0.13), P<0.001)$ than in women $(-0.02 S D(-0.10 ; 0.06)$, $P=0.646)\left(P_{\text {interaction}}=0.002\right.$, Figure 4.3). Additional adjustment for postmenopausal status and/or hormone replacement therapy in women did not materially change these associations (data not shown).

\section{Glycemia and retinal arteriolar dilation and skin hyperemia}

FPG (as a measure of short-term hyperglycemia) was inversely associated with retinal arteriolar \%-dilation and skin \%-hyperemia; per SD higher FPG $(1.7 \mathrm{mmol} / \mathrm{l})$, retinal arteriolar \%-dilation was -0.12 SD $(95 \% \mathrm{Cl}:-0.17 ;-0.07, \mathrm{P}<0.001)$ lower, and skin \%-hyperemia was -0.11SD (-0.17; -0.06, P<0.001) lower (Figure 4.2). In addition, HbA1c (as a measure of long-term hyperglycemia, substituted for FPG) was inversely associated with retinal arteriolar \%-dilation and skin \%-hyperemia; per SD higher HbA1C $(0.9 \mathrm{mmol} / \mathrm{l})$, retinal arteriolar \%-dilation was $-0.14 \mathrm{SD}(95 \% \mathrm{Cl}:-0.19 ;-0.08, \mathrm{P}<0.001)$ lower, and skin \%-hyperemia was -0.12SD (-0.18; -0.06, $\mathrm{P}<0.001)$ lower.

\section{Smoking and retinal arteriolar dilation and skin hyperemia}

Current smoking (versus never smoking) was not associated with retinal arteriolar $\%$-dilation, whereas it was associated with -0.32SD (-0.49; $-0.15, \mathrm{P}<0.001)$ lower skin $\%$-hyperemia (Figure 4.2). Former smoking (versus never smoking) was not associated with retinal arteriolar \%-dilation and skin \%-hyperemia. 


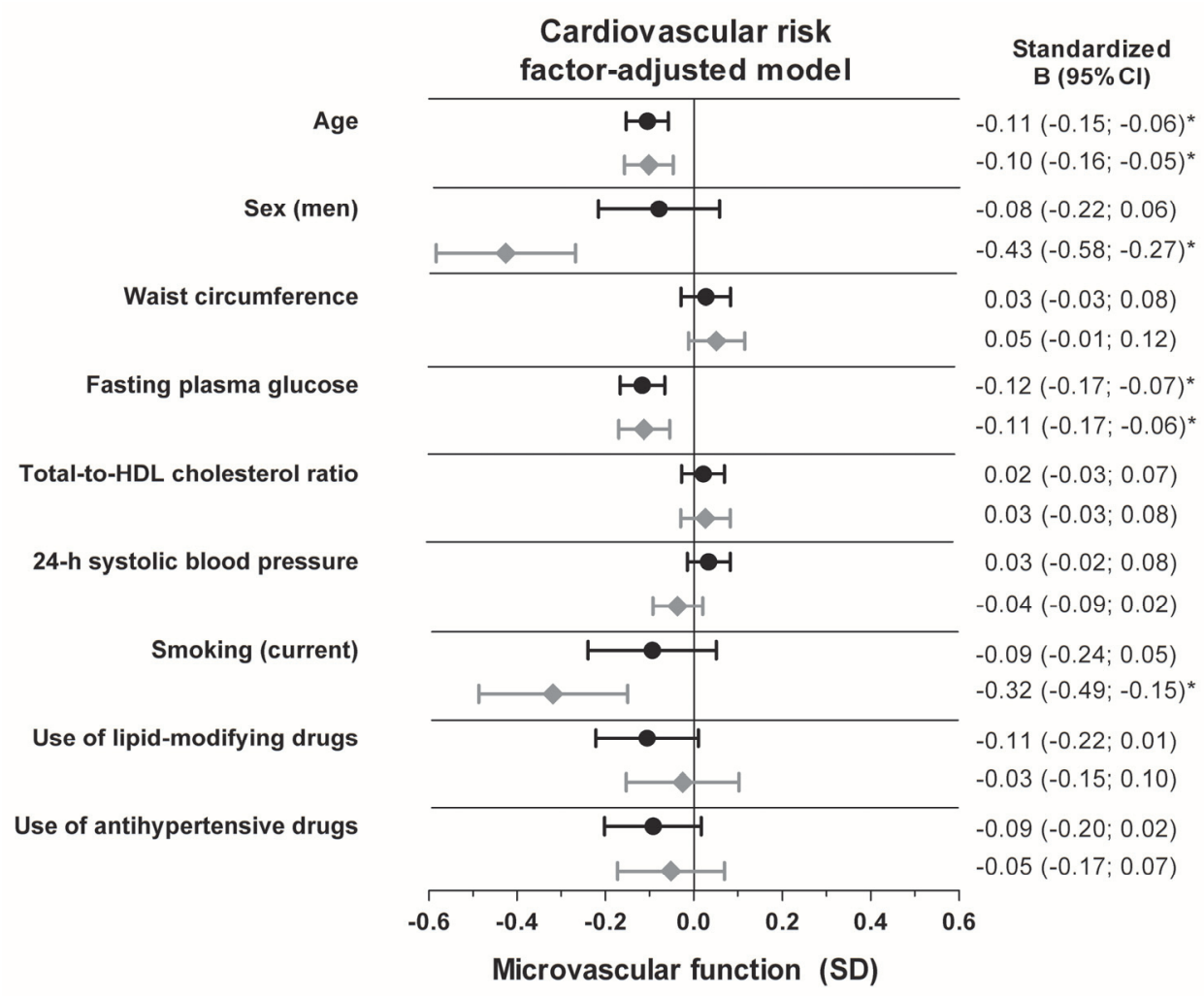

Figure 4.2 Associations of cardiovascular risk factors with retinal arteriolar \%-dilation and skin \%-hyperemia. Point estimates (standardized beta) and $95 \% \mathrm{Cls}$ represent the difference (in SD) in retinal arteriolar \%-dilation (black dots) and skin \%-hyperemia (grey diamonds) per SD increase in the cardiovascular risk factor, men versus women, current smoker versus never smoker, or the use of antihypertensive or lipid-modifying medication versus no use. All associations were adjusted for the other risk factors with multivariate regression. Associations of sex were additionally adjusted for height. ${ }^{*} \mathrm{P}<0.05$. SD, standard deviation; $\mathrm{Cl}$, confidence interval; $\mathrm{HDL}$, high-density lipoprotein.

\section{Blood pressure and retinal arteriolar dilation and skin hyperemia}

24-h systolic blood pressure was not associated with retinal arteriolar \%-dilation or skin \%-hyperemia (Figure 4.2). However, 24-h diastolic blood pressure (substituted for 24-h systolic blood pressure) was associated with retinal arteriolar \%-dilation; per SD higher 24-h diastolic blood pressure $(7.2 \mathrm{mmHg})$, retinal arteriolar \%-dilation was 0.05SD (0.00; 0.10, $\mathrm{P}=0.040)$ greater. 24 -h diastolic blood pressure was not associated with skin \%-hyperemia. In addition, 24-h pulse pressure (substituted for 24-h systolic blood pressure and additionally corrected for 24-h mean arterial pressure) was not associated with retinal arteriolar \%-dilation, but was inversely associated with skin \%-hyperemia. 
Per SD higher 24-h pulse pressure (8.9 mmHg), skin \%-hyperemia was -0.08SD $(-0.14 ;-0.01, P=0.017)$ lower. In addition, use of antihypertensive medication was associated with numerically lower retinal arteriolar \%-dilation (-0.09SD (-0.20; 0.02), $\mathrm{P}=0.099)$ and skin \%-hyperemia (-0.05SD (-0.17; 0.07), $\mathrm{P}=0.396)$.

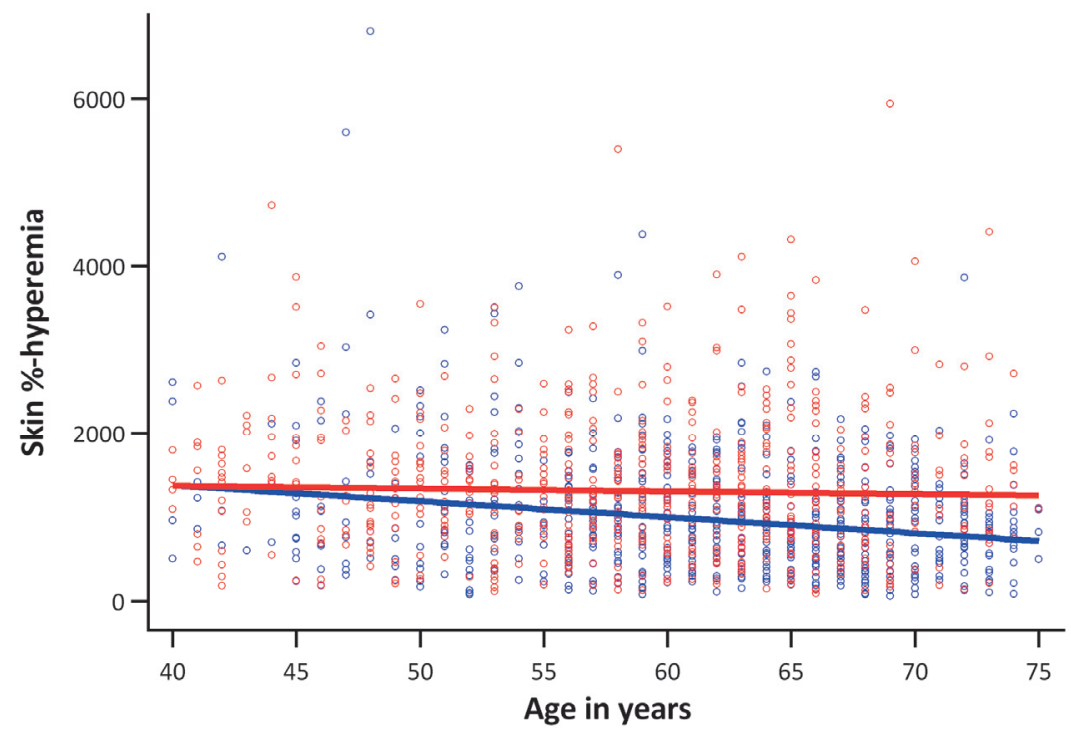

Women B $(95 \% \mathrm{Cl})=-2(-10 ; 6), \mathrm{P}=0.646$

Men B $(95 \% \mathrm{Cl})=-18(-25 ;-11), \mathrm{P}<0.001$

Figure 4.3 Association between age and skin \%-hyperemia according to sex.

Regression coefficients (B) indicate the adjusted mean difference and 95\% confidence interval $(95 \% \mathrm{Cl})$ in skin \%-hyperemia per 1 year increase in age for men (blue line) and women (red line) $\left(P_{\text {interaction }}=0.002\right)$.

\section{Waist, total-to-HDL cholesterol, and lipid-modifying medication use and retinal arteriolar dilation and skin hyperemia}

No significant associations were observed of waist circumference and total-to-HDL cholesterol ratio with retinal arteriolar \%-dilation or skin \%-hyperemia. The use of lipidmodifying medication was associated with numerically lower retinal arteriolar \%-dilation (-0.11SD (-0.22; 0.01), $\mathrm{P}=0.072$ ) (Figure 4.2).

\section{Additional analyses}

Qualitatively similar associations of cardiovascular risk factors with retinal arteriolar \%-dilation and skin \%-hyperemia were observed in a range of additional analyses. First, 
when flicker light-induced increase (in $\mathrm{MU}$ ) in retinal arteriolar diameter from baseline or heat-induced increase in skin blood flow (in PU) from skin baseline were used rather than their percentages (data not shown). Second, when waist circumference was replaced by body mass index. Third, when FPG was substituted by 2-h postload glucose or by T2D (yes/no). Fourth, when smoking status (never, former, current) was replaced by pack-years of smoking (for retinal and skin analyses, data on pack-years of smoking were available in $n=1672$ and $n=1231$ individuals, respectively); pack-years of smoking was inversely associated with skin \%-hyperemia -0.07 SD $(-0.12 ;-0.01, P=0.020)$. No association was found between pack-years of smoking and retinal arteriolar \%-dilation (data not shown). Fifth, after additional adjustment for glucose-lowering medication (although this may be an overadjustment as the use of glucose-lowering medication was part of the definition of T2D (78\% of individuals with T2D used glucose-lowering medication)). Sixth, after additional adjustment for history of cardiovascular disease, eGFR, urinary albumin excretion, and the presence of retinopathy (data not shown, for retinal and skin analyses, data on these additional covariates were available in $n=1884$ and $n=1254$ individuals, respectively). Seventh, when antihypertensive medication was further specified into renin-angiotensin-aldosterone system (RAAS)-inhibiting (with or without other types of antihypertensives) and non-RAAS-inhibiting antihypertensives only (data not shown). RAAS-inhibiting antihypertensives included angiotensinconverting-enzyme inhibitors, angiotensin receptor blockers, and renin blockers. Eight, when individuals with other types of diabetes than T2D were excluded (for retinal and skin analyses, $n=23$ and $n=22$ individuals with other types of diabetes were excluded, respectively). Next, associations of cardiovascular risk factors with retinal arteriolar \%-dilation and skin \%-hyperemia did not differ between individuals with and without $\mathrm{T} 2 \mathrm{D}$, or between women and men (all $\mathrm{P}_{\text {interactions }}>0.10$ ), except as noted above, for the significant interaction, with sex, of the association between age and heat-induced skin hyperemia (Figure 4.3). Last, collinearity diagnostics revealed no multicollinearity in any of the analyses (i.e. all tolerance values $\geq 0.10$ and variance inflation factors $\leq 10$ ).

\section{Discussion}

To our knowledge this is the first population-based study which demonstrated that associations between cardiovascular risk factors and retinal and skin microvascular endothelial function show a pattern that is in part similar to the associations of cardiovascular risk factors and macrovascular function ${ }^{14,16}$. Thus, older age and measures of hyperglycemia were associated with an impaired retinal and skin microvascular endothelium-dependent vasodilation response, independent of other 
cardiovascular risk factors. In addition, male sex and cigarette smoking were associated with impaired heat-induced skin hyperemia. In contrast to associations of obesity, blood pressure, and lipid profile with macrovascular endothelial function ${ }^{16}$, we could not confirm waist circumference, body mass index, 24-h systolic blood pressure, and totalto-HDL cholesterol ratio as determinants of microvascular function. However, (inverse) associations of antihypertensive and lipid-modifying medication use with retinal and skin microvascular function could not be excluded (Figure 4.2). Such associations may imply that previous exposure to elevated blood pressure and dyslipidemia may affect microvascular function more than actual blood pressure and lipid profile. This would also explain why no associations of 24-h systolic blood pressure and total-to-HDL cholesterol ratio with microvascular function were found.

An important mechanism by which cardiovascular risk factors affect large-artery endothelial vasodilation is by impairing nitric oxide bioavailability ${ }^{27}$, possibly in conjunction with adverse effects on other endothelium-dependent vasodilators (e.g. endothelium-derived hyperpolarizing factors) ${ }^{28}$. Similar mechanisms may apply to microvascular function, as microvascular vasodilation also depends on availability of endothelium-dependent vasodilators ${ }^{29,30}$. Therefore, risk factor-associated impairments in flicker light-induced retinal arteriolar dilation and heat-induced skin hyperemia are both likely to be a reflection of microvascular endothelial dysfunction ${ }^{29,30}$, possibly in conjunction with vascular smooth muscle cell dysfunction ${ }^{31,32}$, and/or neuronal dysfunction ${ }^{33,34}$. In addition, both measures of microvascular endothelial function have good reproducibility with intra-individual coefficients of variation of $0.91 \%$ for flicker light-induced retinal arteriolar dilation ${ }^{23}$ and $8.7 \%$ for heat-induced skin hyperemia ${ }^{22}$.

Associations between risk factors and microvascular responses were generally similar regardless of whether the microvascular response was obtained in retina or skin, with two exceptions. First, no difference was found between men and women in retinal arteriolar dilation, whereas heat-induced skin hyperemia was less in men than in women. This is in line with an earlier study which showed that sex differences in retinal arteriolar dilation (i.e. greater in men) were present in young individuals, but diminished after the age of 30 years ${ }^{35}$. This may explain why, in our study with an age span of 40-75 years, no difference was found. Greater retinal arteriolar dilation in younger men, as compared to age-matched women ${ }^{35}$, contrasts with the beneficial effect of female sex hormones on ocular and skin blood flow ${ }^{13,36}$. However, the retinal arteriolar dilation response depends on neurovascular coupling ${ }^{37}$, and sex differences thus may also be influenced by the effects of sex hormones on neurons and astrocytes in the neurovascular coupling unit ${ }^{38}$. Second, retinal arteriolar dilation decreased with age in both men and women, whereas heat-induced skin hyperemia decreased with age only in men (Figure 4.3), which is consistent with an earlier report on macrovascular endothelial 
function $^{39}$. Thus, heat-induced skin hyperemia, as compared to retinal arteriolar dilation, may be protected more by the higher levels of estrogens in women than in men ${ }^{35,40,41}$.

Age was inversely associated with retinal arteriolar and skin microvascular endothelial dysfunction, which is in line with earlier smaller studies 8,13 , and is likely caused by reduced nitric oxide bioavailability ${ }^{42}$. In turn, hyperglycemia may impair microvascular endothelial function through intraendothelial accumulation of glucose, increased oxidative stress, and formation of advanced glycation end products ${ }^{43}$. In addition, microvascular endothelial dysfunction can cause hyperglycemia by impairing the timely access of glucose and insulin to their target tissue ${ }^{44}$ and by impairing insulin secretion $^{45}$.

We hypothesized, but did not find, higher blood pressures to be consistently associated with impaired microvascular function ${ }^{8,11,13}$, especially in the retina, which is known to be sensitive to greater flow pulsatility associated with higher blood pressure and arterial stiffening ${ }^{46}$. A potential explanation is that, in this relatively healthy and well-treated population, the blood pressure range was insufficiently broad for such associations to appear, except that 24-h pulse pressure was inversely associated with heat-induced skin hyperemia. Interestingly, use of antihypertensive medication was associated with numerically lower microvascular function, suggesting that prior exposure to high blood pressure may be important.

Current, but not former smoking was associated with impaired heat-induced skin hyperemia, which suggests that effects of smoking may be reversible. These findings are in line with an earlier report on the detrimental effect of smoking on acetylcholineinduced skin hyperemia ${ }^{10}$. Mechanistically, smoking may induce microvascular dysfunction via increased formation of reactive oxygen species and/or inhibition of nitric oxide synthase activity ${ }^{47}$. As demonstrated previously ${ }^{48}$, we also did not observe a clear association of smoking with impaired retinal arteriolar dilation. Possibly, smoking affects smaller arterioles and capillaries (such as those involved in heat-induced skin hyperemia) more than the relatively large retinal arterioles we assessed ${ }^{48}$.

We hypothesized ${ }^{7,44}$, but did not find, higher waist circumference and body mass index to be associated with impaired microvascular function as assessed here. Importantly, these findings do not imply that other functions of the microcirculation are normal in overweight or obese individuals. Indeed, earlier reports have shown waist and body mass index to be inversely associated with microvascular vasomotion, ${ }^{49}$ post-occlusive reactive hyperemia, ${ }^{50}$ and insulin-mediated vasodilation, ${ }^{44}$ mediated presumably by changes in visceral and perivascular ${ }^{51}$ adipose tissue-derived factors, such as increased tumor necrosis factor- $\alpha$ and free fatty acids, and decreased adiponectin ${ }^{44}$.

We expected ${ }^{9,52}$, but did not find, inverse associations between total-to-HDL cholesterol ratio and retinal and skin microvascular function as assessed here. 
Mechanistically, dyslipidemia may impair microvascular vasodilation via oxidative modifications of low-density lipoprotein cholesterol, which may cause reduced nitric oxide bioavailability, possibly in conjunction with increased expression of endothelin- $1^{52}$. Interestingly, use of lipid-modifying medication was associated with numerically lower retinal arteriolar dilation, suggesting that prior exposure to dyslipidemia may be important.

Strengths of our study include its size and population-based design; the extensive assessment of potential determinants, including 24-h ambulatory blood pressure; the use of two independent methods to directly assess microvascular function in different microvascular beds; and the broad array of additional analyses, which all gave consistent results.

Our study also had limitations. First, the data were cross-sectional. Reverse causality obviously is not an issue for associations with age and sex, but may be especially relevant for hyperglycemia and blood pressure ${ }^{53}$. Therefore, longitudinal studies are needed. Second, we mainly focused on major cardiovascular risk factors as potential determinants; however, we do not claim to have identified all determinants, as there may be others not included in these analyses (e.g. dietary habits and physical activity). Last, the associations observed do not imply that other estimates of microvascular function, or retinal and skin microvascular responses elicited via different stimuli, such as exercise, insulin, or ischemia, relate similarly to cardiovascular risk factors, as compared to flicker light-induced retinal arteriolar dilation and heat-induced skin hyperemia.

This population-based study demonstrated that associations between cardiovascular risk factors and retinal and skin microvascular endothelial function show a pattern that is in part similar to the associations between cardiovascular risk factors and macrovascular endothelial function. Thus, age and measures of hyperglycemia were inversely associated with retinal and skin microvascular endothelium-dependent vasodilation. In addition, male sex and cigarette smoking were associated with impaired heat-induced skin hyperemia. All associations were independent of the other cardiovascular risk factors. We could not confirm waist circumference, body mass index, 24-h systolic blood pressure, and total-to-HDL cholesterol ratio as determinants of these microvascular functions. Possibly, prior exposure to high blood pressure and/or dyslipidemia is important as use of antihypertensive and/or lipid-modifying medication was associated with numerically lower microvascular function. We conclude that impairment of microvascular function may constitute a pathway through which an adverse cardiovascular risk factor pattern may increase risk of diseases, such as heart failure, stroke, and cognitive decline, that in part have a microvascular origin. 


\section{References}

1. Lee JF, Barrett-O'Keefe Z, Garten RS, Nelson AD, Ryan JJ, Nativi JN, Richardson RS and Wray DW. Evidence of microvascular dysfunction in heart failure with preserved ejection fraction. Heart. 2016;102:278-84.

2. Knottnerus IL, Ten Cate H, Lodder J, Kessels F and van Oostenbrugge RJ. Endothelial dysfunction in lacunar stroke: a systematic review. Cerebrovasc Dis. 2009;27:519-26.

3. Santos M, Xekardaki A, Kovari E, Gold G, Bouras C and Giannakopoulos P. Microvascular pathology in latelife depression. J Neurol Sci. 2012;322:46-9.

4. De Silva TM and Faraci FM. Microvascular Dysfunction and Cognitive Impairment. Cell Mol Neurobiol. 2016;36:241-58.

5. Gupta A and Bhatnagar S. Vasoregression: A Shared Vascular Pathology Underlying Macrovascular And Microvascular Pathologies? OMICS. 2015;19:733-53.

6. Zafrani L and Ince C. Microcirculation in Acute and Chronic Kidney Diseases. American Journal of Kidney Diseases. 2015;66:1083-94.

7. de Jongh RT, Serne EH, IJzerman RG, de Vries G and Stehouwer CD. Impaired microvascular function in obesity: implications for obesity-associated microangiopathy, hypertension, and insulin resistance. Circulation. 2004;109:2529-35.

8. Nagel E, Vilser W and Lanzl I. Age, blood pressure, and vessel diameter as factors influencing the arterial retinal flicker response. Invest Ophthalmol Vis Sci. 2004;45:1486-92.

9. Reimann M, Weiss $\mathrm{N}$ and Ziemssen $\mathrm{T}$. Different responses of the retinal and cutaneous microcirculation to transient dysmetabolic conditions. Atheroscler Suppl. 2015;18:1-7.

10. IJzerman RG, Serne EH, van Weissenbruch MM, de Jongh RT and Stehouwer CD. Cigarette smoking is associated with an acute impairment of microvascular function in humans. Clin Sci. 2003;104: 247-52.

11. IJzerman RG, de Jongh RT, Beijk MA, van Weissenbruch MM, Delemarre-van de Waal HA, Serne EH and Stehouwer CD. Individuals at increased coronary heart disease risk are characterized by an impaired microvascular function in skin. Eur J Clin Invest. 2003;33:536-42.

12. Irving RJ, Walker BR, Noon JP, Watt GC, Webb DJ and Shore AC. Microvascular correlates of blood pressure, plasma glucose, and insulin resistance in health. Cardiovasc Res. 2002;53:271-6.

13. Caballero AE, Arora S, Saouaf R, Lim SC, Smakowski P, Park JY, King GL, LoGerfo FW, Horton ES and Veves A. Microvascular and macrovascular reactivity is reduced in subjects at risk for type 2 diabetes. Diabetes. 1999;48:1856-62.

14. Tsao CW and Vasan RS. Cohort Profile: The Framingham Heart Study (FHS): overview of milestones in cardiovascular epidemiology. Int J Epidemiol. 2015;44:1800-13.

15. Chen SM, Tsai TH, Hang CL, Yip HK, Fang CY, Wu CJ and Guo GB. Endothelial dysfunction in young patients with acute ST-elevation myocardial infarction. Heart Vessels. 2011;26:2-9.

16. Witte DR, Westerink J, de Koning EJ, van der Graaf Y, Grobbee DE and Bots ML. Is the association between flow-mediated dilation and cardiovascular risk limited to low-risk populations? J Am Coll Cardiol. 2005;45:1987-93.

17. Aird WC. Endothelial cell heterogeneity. Cold Spring Harb Perspect Med. 2012;2:a006429.

18. Fraser-Bell S, Symes R and Vaze A. Hypertensive eye disease: a review. Clin Exp Ophthalmol. 2017;45: 45-53.

19. Buysschaert M, Medina JL, Bergman M, Shah A and Lonier J. Prediabetes and associated disorders. Endocrine. 2015;48:371-93.

20. Wickman $\mathrm{C}$ and Kramer $\mathrm{H}$. Obesity and kidney disease: potential mechanisms. Semin Nephrol. 2013;33:14-22.

21. Parekh A, Smeeth D, Milner Y and Thure S. The Role of Lipid Biomarkers in Major Depression. Healthcare. 2017;5.

22. Kubli S, Waeber B, Dalle-Ave A and Feihl F. Reproducibility of laser Doppler imaging of skin blood flow as a tool to assess endothelial function. J Cardiovasc Pharmacol. 2000;36:640-8.

23. Nagel E, Vilser W, Fink $A$ and Riemer T. Variance of retinal vessel diameter response to flicker light. A methodical clinical study. Ophthalmologe. 2006;103:114-9. 
24. Schram MT, Sep SJ, van der Kallen CJ, Dagnelie PC, Koster A, Schaper NC, Henry RM and Stehouwer CD. The Maastricht Study: an extensive phenotyping study on determinants of type 2 diabetes, its complications and its comorbidities. Eur J Epidemiol. 2014;29:439-51.

25. Sörensen BM, Houben AJ, Berendschot TT, Schouten JS, Kroon AA, van der Kallen CJ, Henry RM, Koster A, Sep SJ, Dagnelie PC, Schaper NC, Schram MT and Stehouwer CD. Prediabetes and Type 2 Diabetes Are Associated With Generalized Microvascular Dysfunction: The Maastricht Study. Circulation. 2016;134:1339-52.

26. Fitzmaurice $\mathrm{G}$. The meaning and interpretation of interaction. Nutrition. 2000;16:313-4.

27. Puissant C, Abraham P, Durand S, Humeau-Heurtier A, Faure S, Rousseau P and Mahe G. Endothelial function: role, assessment and limits. J Mal Vasc. 2014;39:47-56.

28. Jia G, Durante W and Sowers JR. Endothelium-Derived Hyperpolarizing Factors: A Potential Therapeutic Target for Vascular Dysfunction in Obesity and Insulin Resistance. Diabetes. 2016;65:2118-20.

29. Kellogg DL, Jr., Liu Y, Kosiba IF and O'Donnell D. Role of nitric oxide in the vascular effects of local warming of the skin in humans. J App/ Physiol (1985). 1999;86:1185-90.

30. Lim M, Sasongko MB, Ikram MK, Lamoureux E, Wang JJ, Wong TY and Cheung CY. Systemic associations of dynamic retinal vessel analysis: a review of current literature. Microcirculation. 2013;20:257-68

31. Montero D, Pierce GL, Stehouwer CD, Padilla J and Thijssen DH. The impact of age on vascular smooth muscle function in humans. J Hypertens. 2015;33:445-53.

32. Lacolley P, Regnault V, Nicoletti A, Li Z and Michel JB. The vascular smooth muscle cell in arterial pathology: a cell that can take on multiple roles. Cardiovasc Res. 2012;95:194-204.

33. Minson CT, Berry LT and Joyner MJ. Nitric oxide and neurally mediated regulation of skin blood flow during local heating. J Appl Physiol (1985). 2001;91:1619-26.

34. Falsini B, Riva CE and Logean E. Flicker-evoked changes in human optic nerve blood flow: relationship with retinal neural activity. Invest Ophthalmol Vis Sci. 2002;43:2309-16.

35. Seshadri S, Ekart A and Gherghel D. Ageing effect on flicker-induced diameter changes in retinal microvessels of healthy individuals. Acta Ophthalmol. 2016;94:e35-42.

36. Ogueta SB, Schwartz SD, Yamashita CK and Farber DB. Estrogen receptor in the human eye: influence of gender and age on gene expression. Invest Ophthalmol Vis Sci. 1999;40:1906-11.

37. Riva CE, Logean E and Falsini B. Visually evoked hemodynamical response and assessment of neurovascular coupling in the optic nerve and retina. Prog Retin Eye Res. 2005;24:183-215.

38. Yang SH, Liu R, Perez EJ, Wang $X$ and Simpkins JW. Estrogens as protectants of the neurovascular unit against ischemic stroke. Curr Drug Targets CNS Neurol Disord. 2005;4:169-77.

39. Celermajer DS, Sorensen KE, Spiegelhalter DJ, Georgakopoulos D, Robinson J and Deanfield JE. Aging is associated with endothelial dysfunction in healthy men years before the age-related decline in women. J Am Coll Cardiol. 1994;24:471-6.

40. Cracowski JL. Female hormones and skin microvascular function. Microcirculation. 2011;18:356-7.

41. Arora S, Veves A, Caballaro AE, Smakowski P and LoGerfo FW. Estrogen improves endothelial function. J Vasc Surg. 1998;27:1141-46.

42. Smith AR, Visioli F and Hagen TM. Plasma membrane-associated endothelial nitric oxide synthase and activity in aging rat aortic vascular endothelia markedly decline with age. Arch Biochem Biophys. 2006;454:100-5.

43. Chilelli NC, Burlina S and Lapolla A. AGEs, rather than hyperglycemia, are responsible for microvascular complications in diabetes: a "glycoxidation-centric" point of view. Nutr Metab Cardiovasc Dis. 2013;23:913-9.

44. Jonk AM, Houben AJ, de Jongh RT, Serne EH, Schaper NC and Stehouwer CD. Microvascular dysfunction in obesity: a potential mechanism in the pathogenesis of obesity-associated insulin resistance and hypertension. Physiology. 2007;22:252-60.

45. Hashimoto S, Kubota N, Sato H, Sasaki M, Takamoto I, Kubota T, Nakaya K, Noda M, Ueki K and Kadowaki T. Insulin receptor substrate-2 (Irs2) in endothelial cells plays a crucial role in insulin secretion. Diabetes. 2015;64:876-86.

46. Cheung N, Sharrett AR, Klein R, Criqui MH, Islam FM, Macura KJ, Cotch MF, Klein BE and Wong TY. Aortic distensibility and retinal arteriolar narrowing: the multi-ethnic study of atherosclerosis. Hypertension. 2007; 50:617-22. 
47. Henriksson $P, L U$ Q, Diczfalusy $U$ and Freyschuss A. Immediate effect of passive smoking on microcirculatory flow. Microcirculation. 2014;21:587-92.

48. Garhofer G, Resch H, Sacu S, Weigert G, Schmidl D, Lasta M and Schmetterer L. Effect of regular smoking on flicker induced retinal vasodilatation in healthy subjects. Microvasc Res. 2011;82:351-5.

49. Muris DM, Houben AJ, Kroon AA, Henry RM, van der Kallen CJ, Sep SJ, Koster A, Dagnelie PC, Schram MT and Stehouwer CD. Age, waist circumference, and blood pressure are associated with skin microvascular flow motion: the Maastricht Study. J Hypertens. 2014;32:2439-49.

50. Francischetti EA, Tibirica E, da Silva EG, Rodrigues E, Celoria BM and de Abreu VG. Skin capillary density and microvascular reactivity in obese subjects with and without metabolic syndrome. Microvasc Res. 2011;81:325-30.

51. Yudkin JS, Eringa E and Stehouwer CD. "Vasocrine" signalling from perivascular fat: a mechanism linking insulin resistance to vascular disease. Lancet. 2005;365:1817-20.

52. Seshadri S, Mroczkowska S, Qin L, Patel S, Ekart A and Gherghel D. Systemic circulatory influences on retinal microvascular function in middle-age individuals with low to moderate cardiovascular risk. Acta ophthalmologica. 2015;93:e266-74.

53. Muris DM, Houben AJ, Schram MT and Stehouwer CD. Microvascular dysfunction: an emerging pathway in the pathogenesis of obesity-related insulin resistance. Rev Endocr Metab Disord. 2013;14:29-38. 


\section{Supplemental Material to chapter 4}

\section{Supplemental Methods}

\section{Assessment of retinal and skin microvascular function}

\section{Retinal arteriolar dilation response}

For retinal measurements pupils were dilated with $0.5 \%$ tropicamide and $2.5 \%$ phenylephrine at least 15 minutes prior to the start of the examination. The retinal arteriolar dilation response to flicker light was measured in a dimly lit room by use of the Dynamic Vessel Analyzer (DVA) (Imedos, Jena, Germany). For safety reasons, participants with an intraocular pressure exceeding $30 \mathrm{mmHg}$ were excluded from retinal measurements. Per participant, we randomly measured the left or right eye.

During the measurement, the participant was instructed and encouraged to focus on the tip of a fixated needle inside the retinal camera (FF450; Carl Zeiss GmbH, Jena, Germany), while the fundus of the eye was examined under green measuring light (530-600 nm, illumination of fundus approximately 6500 lux). A straight arteriolar segment of approximately $1.5 \mathrm{~mm}$ in length located 0.5 to 2.0 disc diameter from the margin of the optic disc in the temporal section was examined. When the specific vessel profile was recognized, vessel diameter was automatically and continuously measured for 150 seconds. A baseline recording of 50 seconds was followed by a 40-second flicker light exposure period (flicker frequency $12.5 \mathrm{~Hz}$, bright-to-dark contrast ratio 25:1) followed by a 60-second recovery period. The DVA automatically corrected for alterations in luminance caused by, for example, slight eye movements. During blinks and small eye movements, the registration stopped and restarted once the vessel segment was automatically re-identified.

The integrated DVA software (version 4.51, Imedos) automatically calculated baseline diameter and percentage dilation. Baseline diameter was calculated as the average diameter size of the 20-50 seconds recording and was expressed in measurement units (MU), where $1 \mathrm{MU}$ is equal to $1 \mu \mathrm{m}$ of the Gullstrand eye ${ }^{2}$. Percentage dilation over baseline was based on the average dilation achieved at time points 10 and 40 seconds during the flicker stimulation period. The purpose of taking the average dilation was to account for inter-individual variation in the curve shape during dilation. 


\section{Skin hyperemic response $e^{1}$}

Skin blood flow measurements were performed in a climate-controlled room at $24^{\circ} \mathrm{C}$ with participants in a supine position. Skin blood flow was measured as described previously by means of a laser-Doppler system (Periflux 5000, Perimed, Järfälla, Sweden), equipped with a thermostatic laser-Doppler probe (PF457) at the dorsal side of the wrist of the left hand ${ }^{3}$. The laser-Doppler output was recorded for 25 minutes with a sample rate of $32 \mathrm{~Hz}$, which gives semi-quantitative assessment of skin blood flow expressed in arbitrary perfusion units (PU). Skin blood flow was first recorded unheated for 2 minutes to serve as a baseline. After the 2 minutes of baseline, the temperature of the probe was rapidly and locally increased to $44^{\circ} \mathrm{C}$, and was then kept constant until the end of the registration. The heat-induced skin hyperemic response was expressed as the percentage increase in average PU during the 23 minutes heating phase over the average baseline PU.

\section{Details on the assessment of cardiovascular risk factors}

\section{Glucose metabolism status}

To assess glucose metabolism status, all participants (except those who used insulin) underwent a standardized 2-h 75 gram oral glucose tolerance test (OGTT) after an overnight fast. For safety reasons, participants with a fasting glucose level above $11.0 \mathrm{mmol} / \mathrm{l}$, as determined by a finger prick, did not undergo the OGTT. For these individuals fasting glucose level and information about diabetes medication use were used to assess glucose metabolism status. Glucose metabolism status was defined according to the World Health Organization 2006 criteria as normal glucose metabolism, impaired fasting glucose, impaired glucose tolerance (combined as prediabetes), or type 2 diabetes. Additionally, individuals without type 1 diabetes and no diabetes medication were considered as having type 2 diabetes ${ }^{4}$.

\section{Measures of glycemia}

Venous fasting and postload plasma glucose levels were measured by the enzymatic hexokinase method on two automatic analyzers (i.e. the Beckman Synchron LX20 (Beckman Coulter Inc., USA) for samples obtained between November 2010 and April 2012, and the Roche Cobas 6000 (Roche Diagnostics, Mannheim, Germany) for samples obtained thereafter). Glycated hemoglobin A1c (HbA1c) was determined by ion-exchange high-performance liquid chromatography ${ }^{4}$. 


\section{Measures of blood pressure}

Participants underwent 24-h blood pressure monitoring (WatchBP03, Microlife AG, Widnau, Switzerland) with an appropriate-sized cuff placed around their non-dominant $\mathrm{arm}^{4}$. The device was programmed to take blood pressure readings every 15 minutes during daytime (8 a.m. - 11 p.m.) and every 30 minutes during the night (11 p.m. -8 a.m.). According to recommendations of the British Hypertension society ${ }^{5}$, mean 24-h blood pressure measurements were calculated only if there were more than 14 valid blood pressure measurements during daytime and more than 7 valid measurements during nighttime. 24-h mean arterial pressure was calculated as 24-h diastolic blood pressure + (1/3* (24-h systolic blood pressure - 24-h diastolic blood pressure)). 24-h pulse pressure was calculated as 24-h systolic blood pressure - 24-h diastolic blood pressure. The use of antihypertensive medication was assessed during a medication interview where generic name, dose, and frequency were registered ${ }^{4}$.

\section{Measures of lipid profile}

Serum concentrations of total cholesterol, high-density lipoprotein (HDL) cholesterol, and triglycerides were measured using an automatic analyzer (Beckman Synchron LX20, Beckman Coulter Inc. Brea, USA). The use of lipid-modifying medication was assessed during a medication interview where generic name, dose, and frequency were registered ${ }^{4}$.

\section{Details on the assessment of covariates}

We measured height, weight, office blood pressure, serum creatinine and cystatin $C^{6}$, and 24-h urinary albumin excretion (twice) as described previously ${ }^{4}$. Body mass index was calculated as weight $(\mathrm{kg})$ divided by height $\left(\mathrm{m}^{2}\right)$. Estimated glomerular filtration rate (eGFR; in $\mathrm{ml} / \mathrm{min} / 1.73 \mathrm{~m}^{2}$ ) was calculated with the Chronic Kidney Disease Epidemiology Collaboration (CDK-epi) equation based on both serum creatinine and serum cystatin $\mathrm{C}^{11}$. The presence of retinopathy was based on fundus photographs taken with an auto fundus camera (Model AFC-230, Nidek, Gamagori, Japan). History of cardiovascular disease was assessed by web-based questionnaires and was defined as a history of myocardial infarction, stroke, or vascular surgery (including angioplasty) of coronary, carotid, abdominal aortic, or peripheral arteries ${ }^{4}$. 
Statistical analysis

Differences in general characteristics among individuals in the study populations and individuals excluded due to missing values were compared by Analyses of Variance (ANOVA) for continuous variables and $x^{2}$-test for categorical variables.

Supplemental Tables 


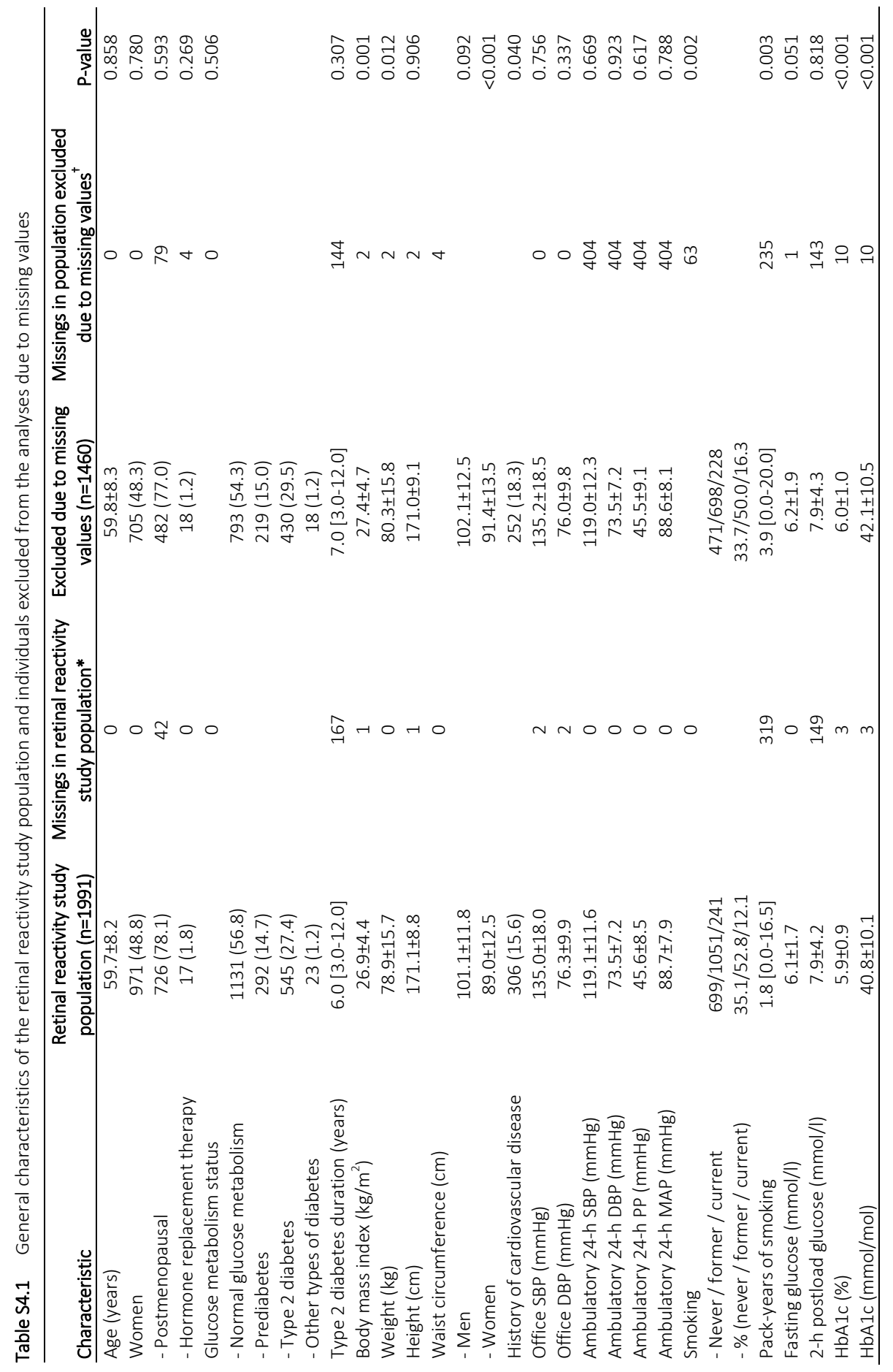




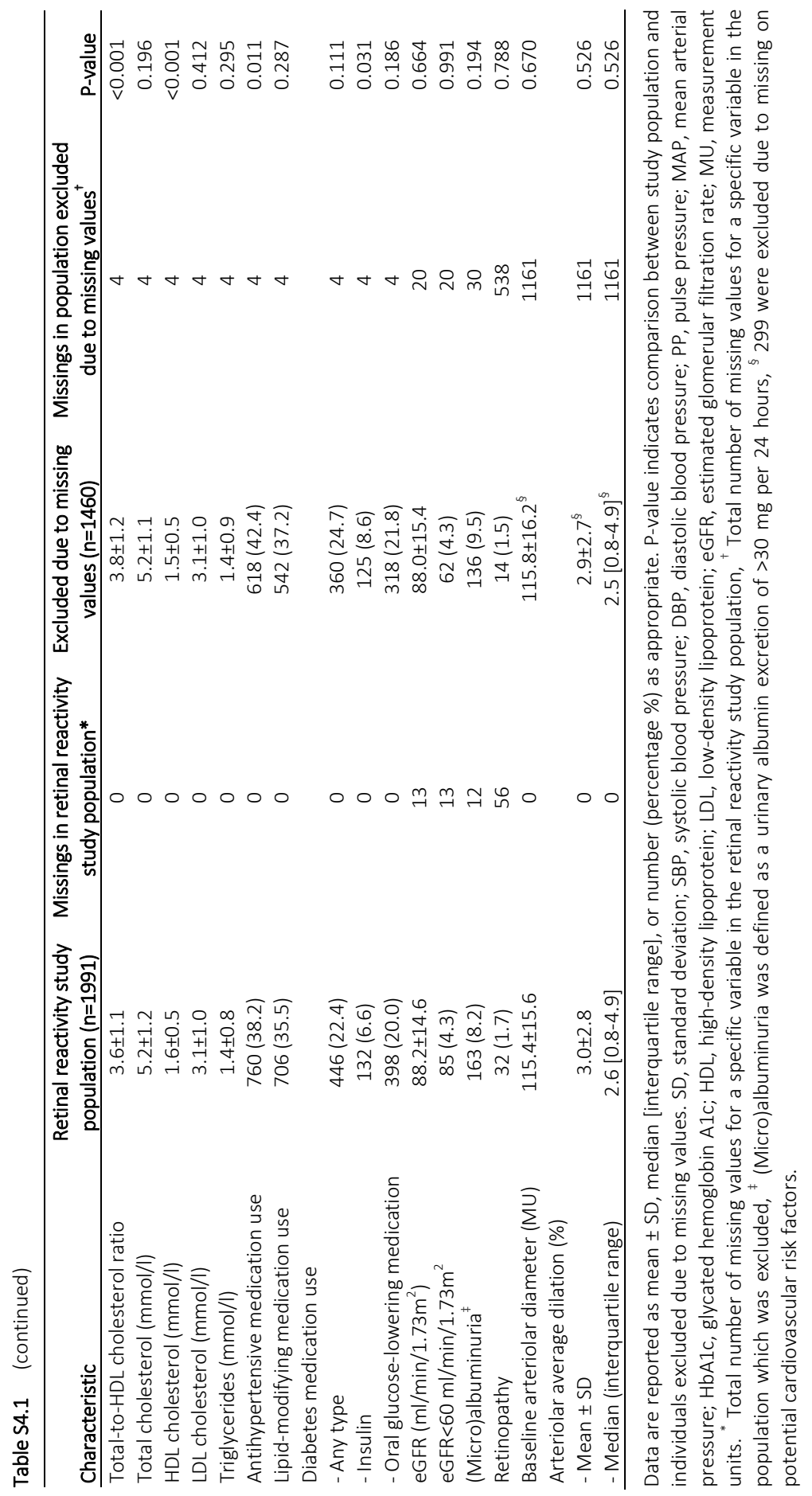




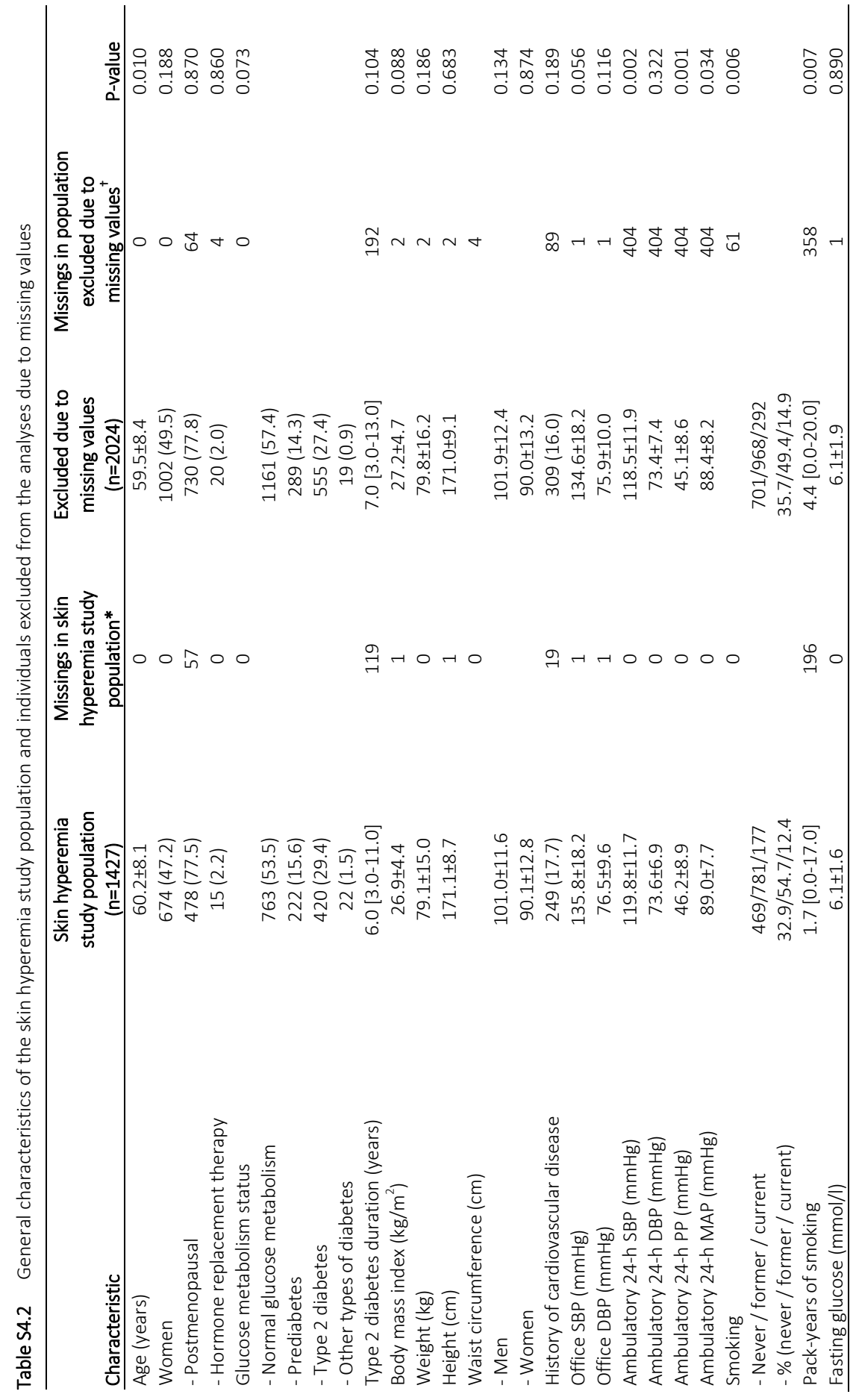




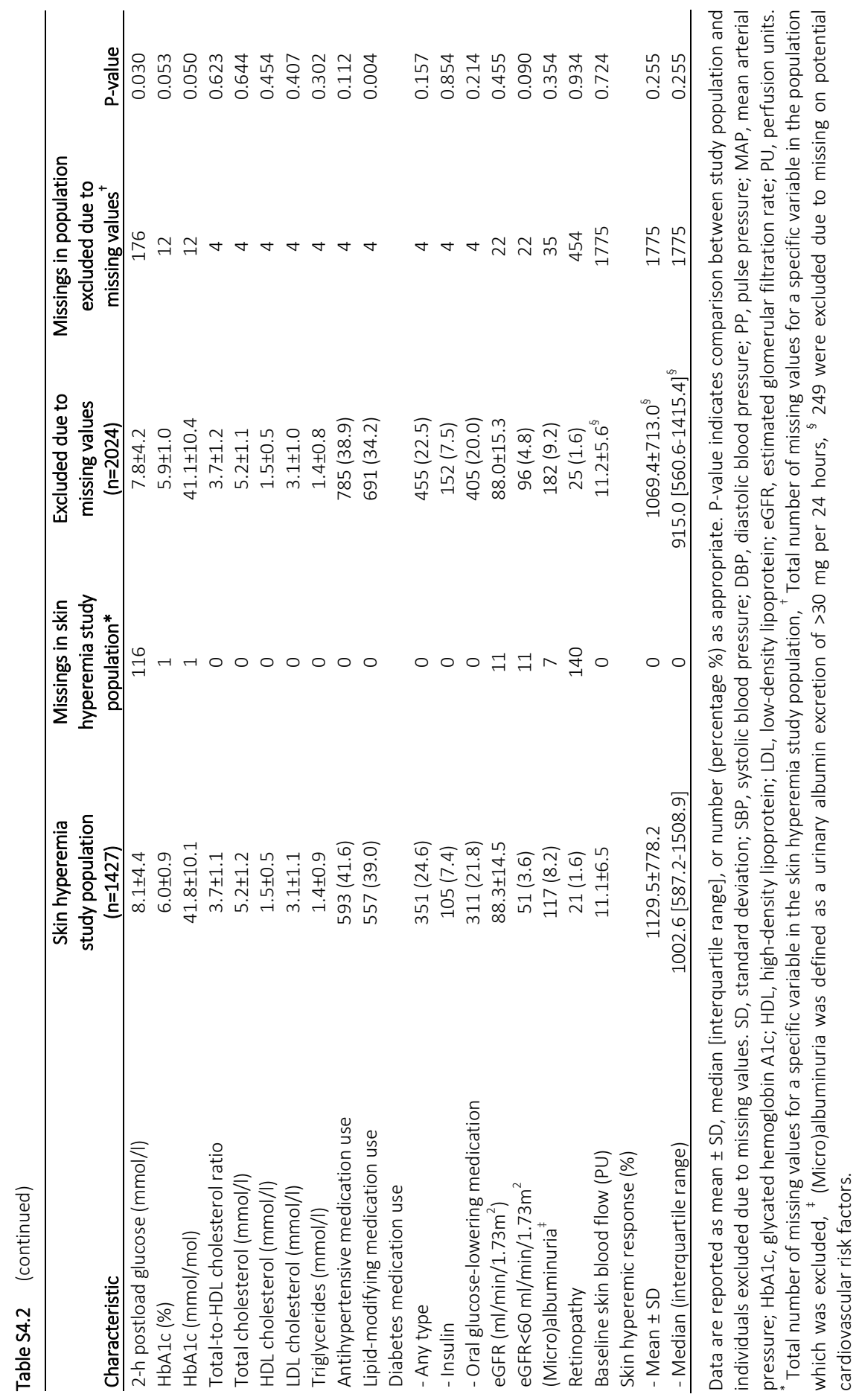




\section{References}

1. Sörensen BM, Houben AJ, Berendschot TT, Schouten JS, Kroon AA, van der Kallen CJ, Henry RM, Koster A, Sep SJ, Dagnelie PC, Schaper NC, Schram MT and Stehouwer CD. Prediabetes and Type 2 Diabetes Are Associated With Generalized Microvascular Dysfunction: The Maastricht Study. Circulation. 2016;134:1339-52.

2. Nagel E, Vilser W, Fink A and Riemer T. Variance of retinal vessel diameter response to flicker light. A methodical clinical study. Ophthalmologe. 2006;103:114-9.

3. Muris DM, Houben AJ, Kroon AA, Henry RM, van der Kallen CJ, Sep SJ, Koster A, Dagnelie PC, Schram MT and Stehouwer CD. Age, waist circumference, and blood pressure are associated with skin microvascular flow motion: the Maastricht Study. J Hypertens. 2014;32:2439-49.

4. Schram MT, Sep SJ, van der Kallen CJ, Dagnelie PC, Koster A, Schaper NC, Henry RM and Stehouwer CD. The Maastricht Study: an extensive phenotyping study on determinants of type 2 diabetes, its complications and its comorbidities. Eur J Epidemiol. 2014;29:439-51.

5. O'Brien E, Coats A, Owens P, Petrie J, Padfield PL, Littler WA, de Swiet $M$ and Mee F. Use and interpretation of ambulatory blood pressure monitoring: recommendations of the British hypertension society. BMJ. 2000;320:1128-34.

6. Martens RJ, Henry RM, Houben AJ, van der Kallen CJ, Kroon AA, Schalkwijk CG, Schram MT, Sep SJ, Schaper NC, Dagnelie PC, Muris DM, Gronenschild EH, van der Sande FM, Leunissen KM, Kooman JP and Stehouwer CD. Capillary Rarefaction Associates with Albuminuria: The Maastricht Study. J Am Soc Nephrol. 2016;27:3748-57. 


\section{Chapter 5}

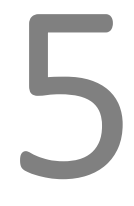

Higher levels of daily physical activity are associated with better skin microvascular function in type 2 diabetes: The Maastricht Study

Ben M. Sörensen, Alfons J.H.M. Houben, Annemarie Koster, Tos T.J.M. Berendschot, Jan S.A.G. Schouten, Abraham A. Kroon, Carla J.H. van der Kallen, Ronald M.A. Henry, Hans. H.C.M. Savelberg, Martien C.J.M van Dongen, Simone J.P.M. Eussen, Julianne D. van der Berg, Nicolaas C. Schaper, Miranda T. Schram, Coen D.A. Stehouwer 


\section{Abstract}

\section{Objective}

Low levels of physical activity and high levels of sedentary time are associated with macrovascular diseases and act in part through inducing large-artery endothelial dysfunction. We investigated whether low levels of physical activity and high levels of sedentary time also associate with microvascular endothelial function.

\section{Methods}

In The Maastricht Study, a population-based cohort study enriched with type 2 diabetes (T2D) ( $n=1298,47.3 \%$ women, aged $60.2 \pm 8.1$ years, 29.5\% T2D), we determined microvascular function as heat-induced skin hyperemia and flicker light-induced retinal arteriolar \%-dilation. Physical activity and sedentary time were assessed by an accelerometer worn 24 hours a day for 8 consecutive days. Multivariable linear regression analyses were used to examine associations of total and higher-intensity physical activity and total sedentary time with skin and retinal microvascular function adjusted for major cardiovascular risk factors. In addition, we investigated whether associations were stronger in T2D.

\section{Results}

Associations of total and higher-intensity physical activity with skin hyperemia were stronger in individuals with as compared to those without $T 2 D\left(P_{\text {interactions }}<0.10\right)$. In individuals with $T 2 D$, total and higher-intensity physical activity were independently associated with greater heat-induced skin hyperemia (regression coefficients per hour increase were 10 perfusion units (PU) (95\% Cl: 1; 18, P=0.026) and $36 \mathrm{PU}(14 ; 58, \mathrm{P}=0.002)$, respectively). Total and higher-intensity physical activity and total sedentary time were not associated with heat-induced skin hyperemia in individuals without T2D, or with retinal arteriolar \%-dilation.

\section{Conclusions}

Higher levels of total and higher-intensity physical activity were associated with greater skin microvascular vasodilation in individuals with, but not in those without T2D. These findings suggest that increasing habitual daily physical activity should be investigated as a means to improving microvascular function in T2D, with the ultimate goal of reducing risk of (partly) microvascular diseases such as heart failure, (lacunar) stroke, depression, cognitive decline, retinopathy, chronic kidney failure, and neuropathy. 


\section{Introduction}

Microvascular dysfunction is an important underlying mechanism in common diseases, such as heart failure ${ }^{1}$, (lacunar) stroke ${ }^{2}$, depression ${ }^{3}$, cognitive decline ${ }^{4}$, retinopathy ${ }^{5}$, chronic kidney disease ${ }^{6}$, and neuropathy ${ }^{5}$, which occur in the general population and more frequently in individuals with type 2 diabetes (T2D) ${ }^{7}$. It is therefore important to identify, in particular modifiable, factors that contribute to the development of microvascular dysfunction, as these can be targeted in preventive strategies.

Physical activity may be one such modifiable risk factor, as may sedentary behavior, which refers to activities that do not increase energy expenditure substantially above the resting level, such as sitting, watching television, and using the computer ${ }^{8}$.

Indeed, low levels of physical activity and high levels of sedentary time have been shown to be associated with macrovascular diseases ${ }^{9}$ (e.g. stroke, myocardial infarction, and peripheral arterial disease) and may act through inducing large-artery endothelial dysfunction ${ }^{10,11}$. Low levels of physical activity and high levels of sedentary time have also been shown to be associated with diseases that may be partly or wholly of microvascular origin ${ }^{12-18}$. Thus, we hypothesized that low levels of physical activity and high levels of sedentary time may affect microvascular (endothelial) function. In support, several small (mostly intervention) studies have shown beneficial effects of physical activity $^{19-22}$, and adverse effects of sedentary behavior ${ }^{19,23-26}$, on microvascular function. However, it is unclear whether daily life habitual physical activity, as compared to exercise programs ${ }^{20,22}$, also beneficially affects microvascular function. Earlier studies were conducted in small numbers of individuals ${ }^{19-26}$, which limits translation to the general population, or were based on self-reported measures of physical activity and sedentary behavior ${ }^{27}$ which are known to be less precise and valid than objective measures ${ }^{28}$.

Mechanistically, higher levels of physical activity and lower levels of sedentary time are thought to increase nitric oxide bioavailability ${ }^{29,30}$, presumably in conjunction with beneficial effects on low-grade inflammation ${ }^{31,32}$, both of which may improve microvascular (endothelial) function ${ }^{33}$. As hyperglycemia has detrimental effects on nitric oxide bioavailability ${ }^{34}$ and microvascular (endothelial) function ${ }^{35}$, the associations of physical activity and sedentary behavior with microvascular (endothelial) function may differ between (i.e. may be stronger in) individuals with as compared to those without T2D.

In view of these considerations, we investigated, in a population-based study with oversampling of individuals with T2D, whether objectively measured physical activity and sedentary behavior were associated with skin microvascular and retinal arteriolar (endothelial) function. In addition, we examined whether these associations differed 
between individuals with and without T2D. We chose skin and retina for 2 reasons. First, these sites are easily accessible, enabling direct and reproducible ${ }^{36,37}$ assessment of microvascular (endothelial) function, as measured by heat-induced skin hyperemia and flicker light-induced retinal arteriolar dilation. Second, skin and retina may differ in their associations with physical activity and sedentary behavior, with skin potentially showing stronger associations. This is because skin is important in disseminating exerciseproduced heat ${ }^{38}$, whereas retinal blood flow, in contrast, has been shown to be relatively stable during exercise ${ }^{39,40}$, presumably to maintain visual performance ${ }^{41}$.

\section{Methods}

\section{Study population and design}

We used data from The Maastricht Study, an observational prospective populationbased cohort study. The rationale and methodology have been described previously ${ }^{42}$. In brief, the study focuses on the etiology, pathophysiology, complications, and comorbidities of T2D and is characterized by an extensive phenotyping approach. Eligible for participation were all individuals aged between 40 and 75 years and living in the southern part of the Netherlands. Participants were recruited through mass media campaigns and from the municipal registries and the regional Diabetes Patient Registry via mailings. Recruitment was stratified according to known T2D status, with an oversampling of individuals with T2D, for reasons of efficiency. The present report includes cross-sectional data from the first 3451 participants, who completed the baseline survey between November 2010 and September 2013. The examinations of each participant were performed within a time window of three months. The study has been approved by the institutional medical ethical committee (NL31329.068.10) and the Minister of Health, Welfare and Sports of the Netherlands (Permit 131088-105234-PG). All participants gave written informed consent.

\section{Assessment of glucose metabolism status}

To assess glucose metabolism status, all participants (except those who used insulin) underwent a standardized 2-h 75 gram oral glucose tolerance test (OGTT) after an overnight fast. For safety reasons, participants with a fasting glucose level above $11.0 \mathrm{mmol} / \mathrm{l}$, as determined by a finger prick, did not undergo the OGTT. For these individuals fasting glucose level and information about diabetes medication use were used to assess glucose metabolism status. Glucose metabolism status was defined according to the World Health Organization 2006 criteria as normal glucose metabolism, 
impaired fasting glucose, impaired glucose tolerance (combined as prediabetes), or T2D. Individuals without type 1 diabetes on glucose-lowering medication were classified as having $T 2 \mathrm{D}^{42}$.

\section{Assessment of microvascular function}

All participants were asked to refrain from smoking and drinking caffeine-containing beverages three hours before the measurement. A light meal (breakfast and (or) lunch), low in fat content, was allowed if taken at least 90 minutes prior to the start of the measurements.

Skin blood flow measurements were performed in a climate-controlled room at $24^{\circ} \mathrm{C}$ with participants in a supine position. Skin blood flow was measured as described previously by means of a laser-Doppler system (Periflux 5000, Perimed, Järfälla, Sweden), equipped with a thermostatic laser-Doppler probe (PF457) at the dorsal side of the wrist of the left hand. The laser-Doppler output was recorded for 25 minutes with a sample rate of $32 \mathrm{~Hz}$, which gives semi-quantitative assessment of skin blood flow expressed in arbitrary perfusion units (PU). Skin blood flow was first recorded unheated for 2 minutes to serve as a baseline. After the 2 minutes of baseline, the temperature of the probe was rapidly and locally increased to $44^{\circ} \mathrm{C}$, and was then kept constant until the end of the registration. In contrast to earlier work ${ }^{43}$, heat-induced skin hyperemia was expressed not as a percentage of baseline but as perfusion during the 23 minutes heating phase (in PU), because spurious associations between determinants and heat-induced skin hyperemia expressed as percentage may occur when determinants are associated with baseline skin blood flow (as was the case here; see Supplemental Results and Supplemental Table S5.3). To investigate associations of heat-induced skin hyperemia independently of associations with baseline skin blood flow, we adjusted for baseline flow in the regression models (this will not lead to autocorrelation because there was only a weak association between baseline skin blood flow and heat-induced skin hyperemia) ${ }^{44,45}$.

For retinal measurements pupils were dilated with $0.5 \%$ tropicamide and $2.5 \%$ phenylephrine at least 15 minutes prior to the start of the examination. Retinal arteriolar vasodilation to flicker light exposure was measured by the Dynamic Vessel Analyzer (Imedos, Jena, Germany). Briefly, a baseline recording of 50 seconds was followed by 40-second flicker light exposure followed by a 60-second recovery period. Baseline diameter was calculated as the average diameter size of the 20-50 seconds recording and was expressed in measurement units. As determinants were not associated with retinal arteriolar baseline diameter (see Supplemental Results and Supplemental Table S5.4), flicker light-induced retinal arteriolar dilation was expressed as a percentage over baseline, based on the average dilation achieved at time points 10 and 40 seconds 
during the flicker stimulation period. The measurement has extensively been described previously ${ }^{43}$ and more details are provided in the Supplemental Material.

\section{Assessment of physical activity and sedentary behavior}

Daily activity levels and patterns were measured using the activPAL3 ${ }^{\text {TM }}$ physical activity monitor (PAL technologies, Glasgow, UK) as has been described previously ${ }^{46}$ and in the Supplemental Methods. Participants were asked to wear the accelerometer for 8 consecutive days, without removing the device at any time. The total amount of physical activity (stepping time) was based on the stepping posture and calculated as the mean time spent stepping during waking time per day, standing time was not included. The method used to determine waking time has been described elsewhere ${ }^{46}$. Higherintensity physical activity was defined as hours with a step frequency $>110$ steps/min during waking time ${ }^{47,48}$; again standing time was not included. The total amount of sedentary time was based on the sedentary posture (sitting or lying) and calculated as the mean time spent in a sedentary position during waking time per day ${ }^{46}$.

\section{Measurement of covariates}

History of cardiovascular disease, smoking status (never, former, current), alcohol consumption, level of education, occupational status, daily energy intake, mobility limitation, and duration of diabetes were assessed by questionnaire ${ }^{42}$. Mobility limitation was obtained from the EuroQol-5D questionnaire and was defined as having difficulty walking 500 meter or climbing the stairs in the previous week. Energy intake was obtained from a food frequency questionnaire and calculated as the mean energy intake (kcal) per day. Use of antihypertensive, lipid-modifying, and glucose-lowering medication was assessed during a medication interview where generic name, dose, and frequency were registered ${ }^{49}$. We measured height, weight, waist circumference, office and ambulatory 24-h blood pressure, plasma glucose levels, serum creatinine, 24-h urinary albumin excretion (twice), glycated hemoglobin A1c (HbA1c), and plasma lipid profile as described elsewhere ${ }^{42}$. Body mass index was calculated as weight $(\mathrm{kg})$ divided by height $\left(\mathrm{m}^{2}\right)$. Estimated glomerular filtration rate (eGFR; in $\mathrm{ml} / \mathrm{min} / 1.73 \mathrm{~m}^{2}$ ) was calculated with the Chronic Kidney Disease Epidemiology Collaboration (CDK-epi) equation based on both serum creatinine and serum cystatin $C^{50}$. The presence of retinopathy was based on fundus photographs taken with an auto fundus camera (Model AFC-230, Nidek, Gamagori, Japan). 


\section{Statistical analysis}

All analyses were performed with Statistical Package for Social Sciences version 23.0 (IBM SPSS, Armonk, USA). Multivariable linear regression analyses were used to investigate the associations of total physical activity (h/day), higher-intensity physical activity (h/day), and total sedentary time (h/day) with baseline skin blood flow, baseline retinal arteriolar diameter, heat-induced skin hyperemia, and flicker light-induced retinal arteriolar \%-dilation. Model 1 was adjusted for age, sex, glucose metabolism status, baseline skin blood flow (only for analyses of heat-induced skin hyperemia), and waking time (to exclude the possibility that estimated effects were influenced by differences in waking hours). Model 2 was additionally adjusted for potential confounders such as body mass index, educational level, mobility limitation, office systolic blood pressure, total-to-high-density lipoprotein (HDL) cholesterol ratio, triglycerides, smoking status, alcohol consumption, the use of antihypertensive- and/or lipid-modifying medication, and history of cardiovascular disease. In model $3 a$ higher-intensity physical activity was additionally adjusted for total sedentary time. In model $3 \mathrm{~b}$ total sedentary time was additionally adjusted for higher-intensity physical activity. Data were expressed as regression coefficients and corresponding $95 \%$ confidence intervals $(95 \% \mathrm{Cl})$.

The Maastricht Study by design oversampled individuals with T2D, and we therefore were able to investigate potential interactions between total physical activity, higherintensity physical activity, and total sedentary time with T2D by adding interaction terms to the regression models. In stratified analyses in individuals without T2D, model 1 was additionally adjusted for prediabetes status. To assess whether associations differed by sex, interactions between total physical activity, higher-intensity physical activity, and total sedentary time with sex were investigated. In these analyses, interaction terms were the product of total physical activity, higher-intensity physical activity, or total sedentary time and T2D or sex, respectively.

Collinearity diagnostics (i.e. tolerance $<0.10$ and/or variance inflation factor $>10$ ) were used to detect multicollinearity between covariates. A P-value $<0.05$ was considered statistically significant except for interaction analyses, where a $P_{\text {interaction }}<0.10$ was considered statistically significant. 


\section{Results}

\section{Characteristics of study population}

From the initial 3451 participants included, those with other types of diabetes than T2D were excluded $(n=41)$. Of the remaining 3410 participants, heat-induced skin hyperemia data were available in 1647 . The reasons for missing data were logistical $(n=1650)$ or insufficient measurement quality $(n=113)$. Accelerometry variables were missing in 272 participants and data on potential confounders were missing in 77 participants. The heat-induced skin hyperemia study population thus consisted of 1298 participants. Retinal arteriolar reactivity data were available in 2261 participants. The reasons for missing data were logistical ( $n=882)$, insufficient measurement quality $(n=208)$, or contraindications $(n=59)$. Accelerometry variables were missing in 330 participants, particularly due to device availability $(n=233)$, and data on potential confounders were missing in 84 participants. The retinal arteriolar reactivity study population thus consisted of 1847 participants (Figure 5.1 shows the flow chart).

General characteristics of skin reactivity study population are shown in Table 5.1, according to tertiles of total physical activity. This study population had a mean age \pm standard deviation (SD) of $60.2 \pm 8.1$ years, of whom $46.3 \%$ were women and $29.5 \%$ had T2D (oversampled by design). In addition, when compared to individuals in the lowest tertile of total physical activity, those in the middle and highest tertiles were on average younger, had lower glucose levels, and were less likely to have had a history of cardiovascular disease (Table 5.1). The skin study population overlapped for $73 \%$ with the retinal study population. Both populations were comparable with regard to age, sex, and cardiometabolic risk profile (Table 5.1). Individuals excluded from the analyses due to missing data on skin or retinal reactivity measurements, accelerometry measurements, or covariates were also highly comparable to individuals included in the study populations with regard to age, sex, and cardiometabolic risk profile (Supplemental Tables S5.1 and S5.2). 


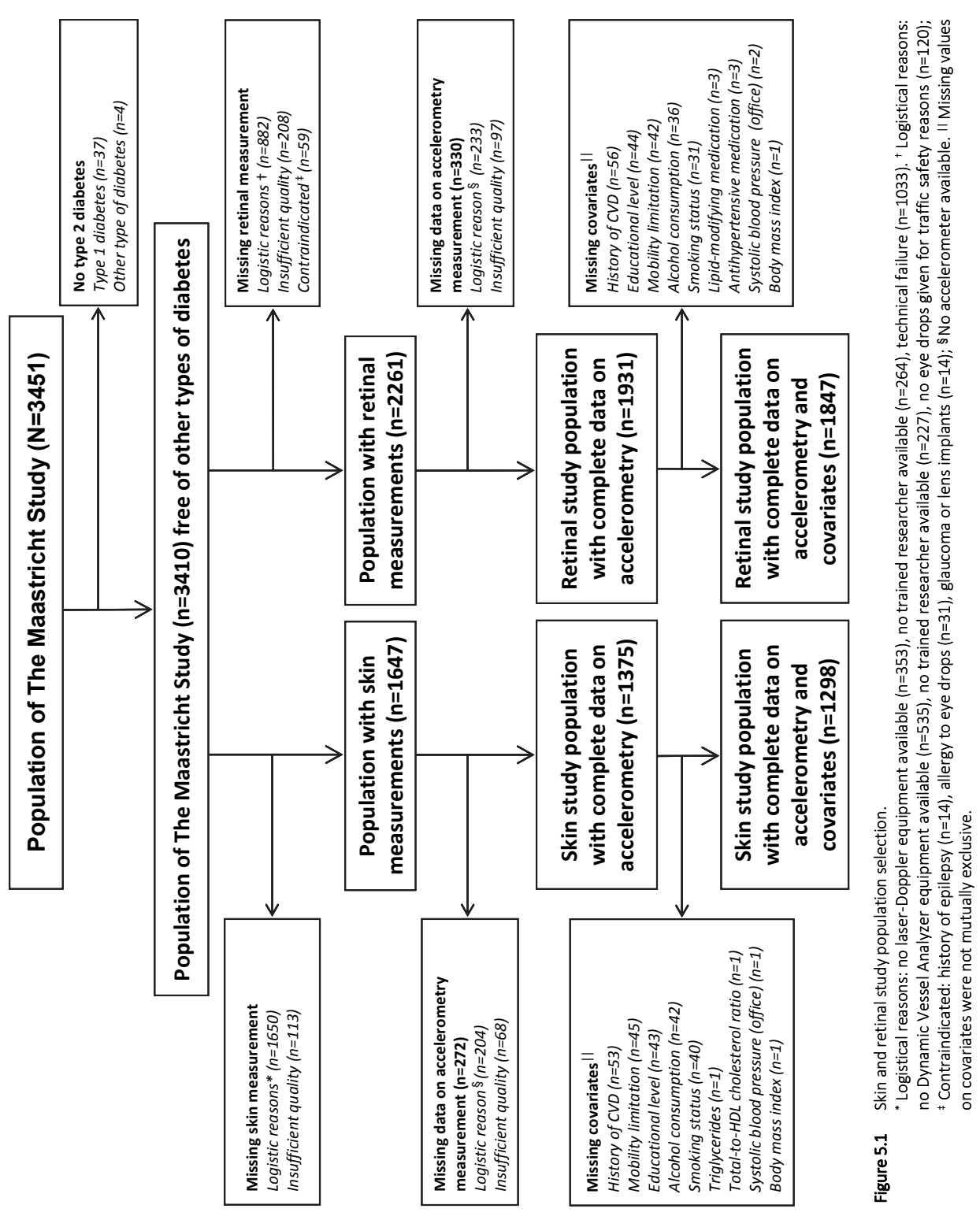




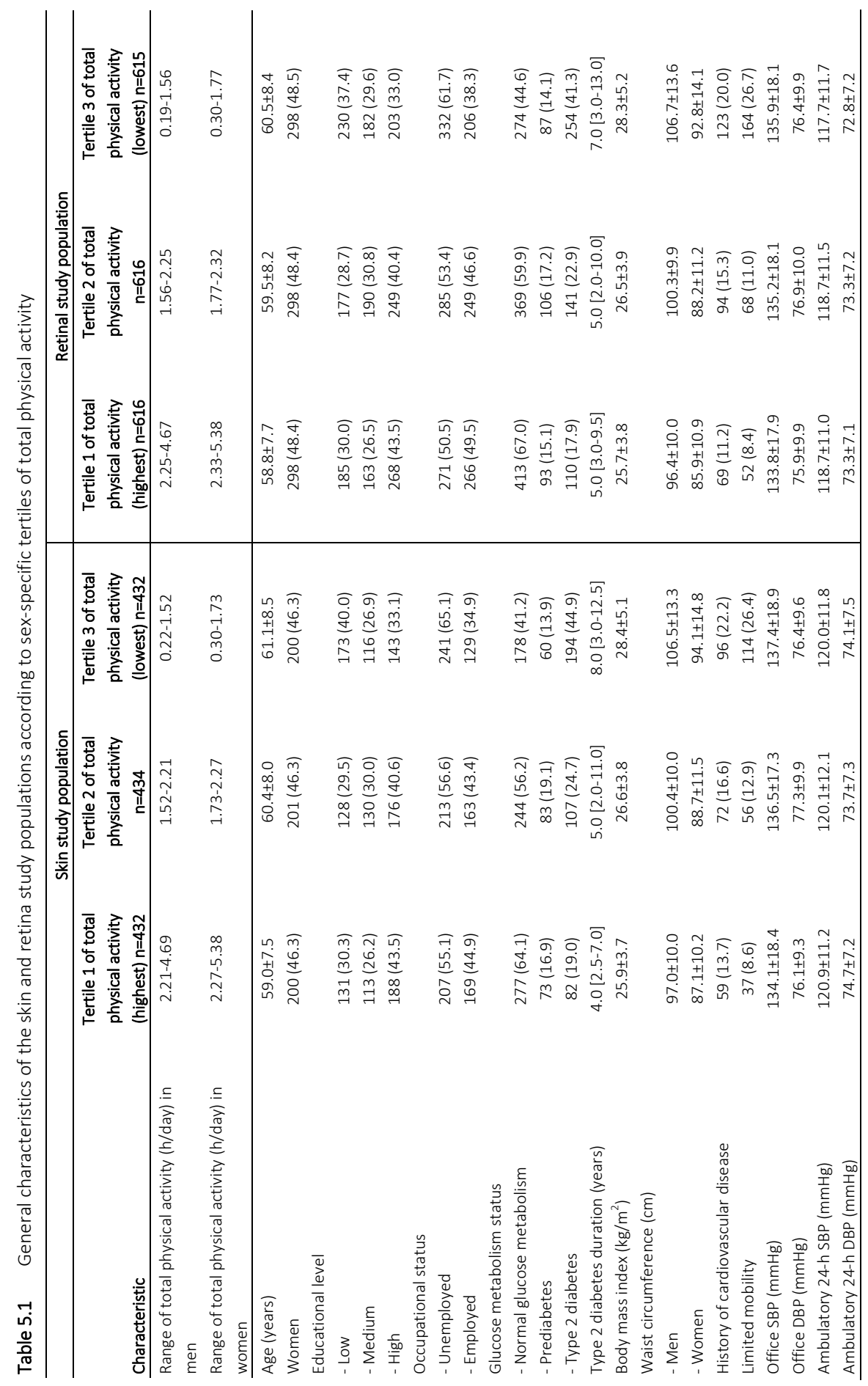




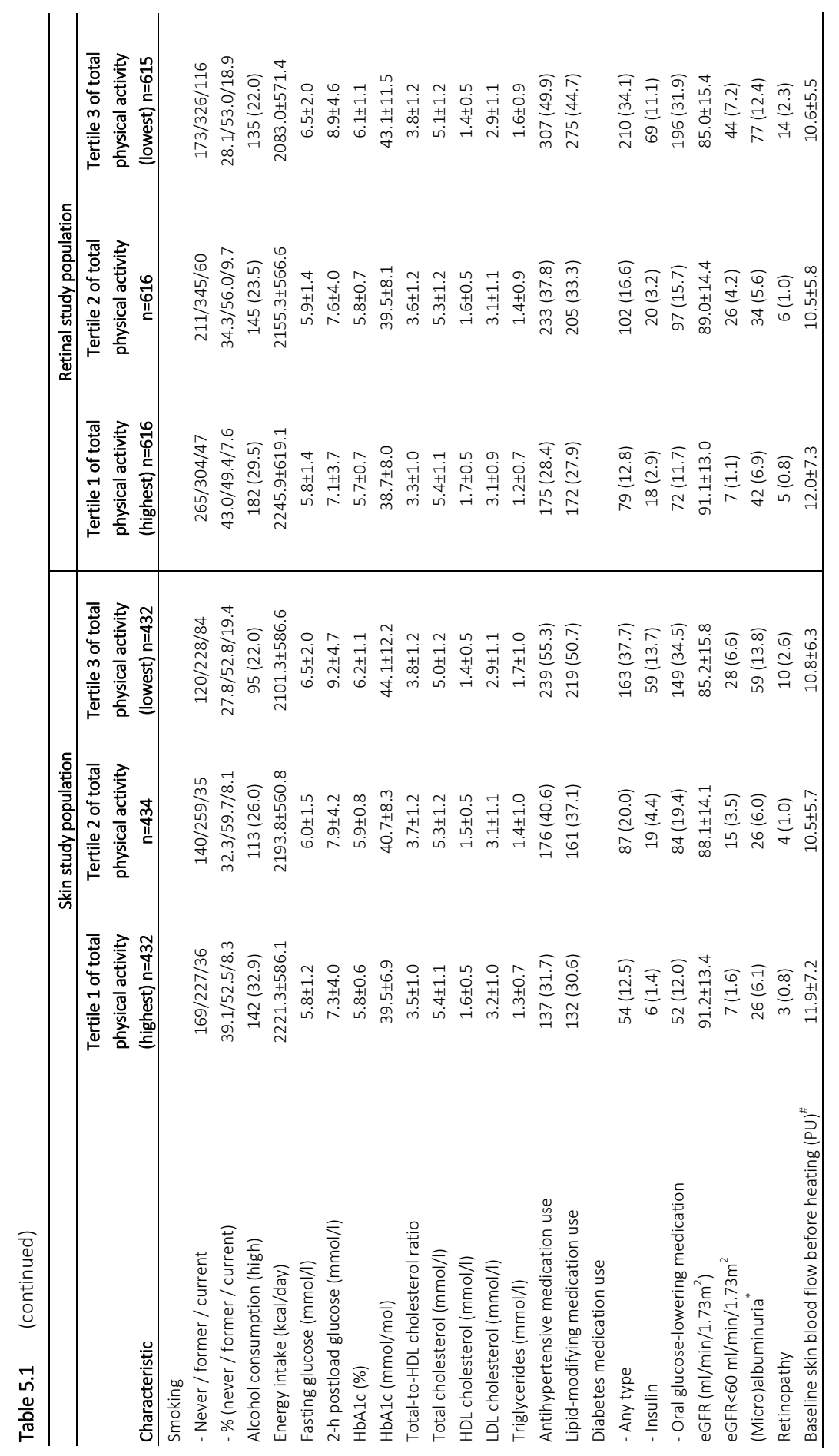




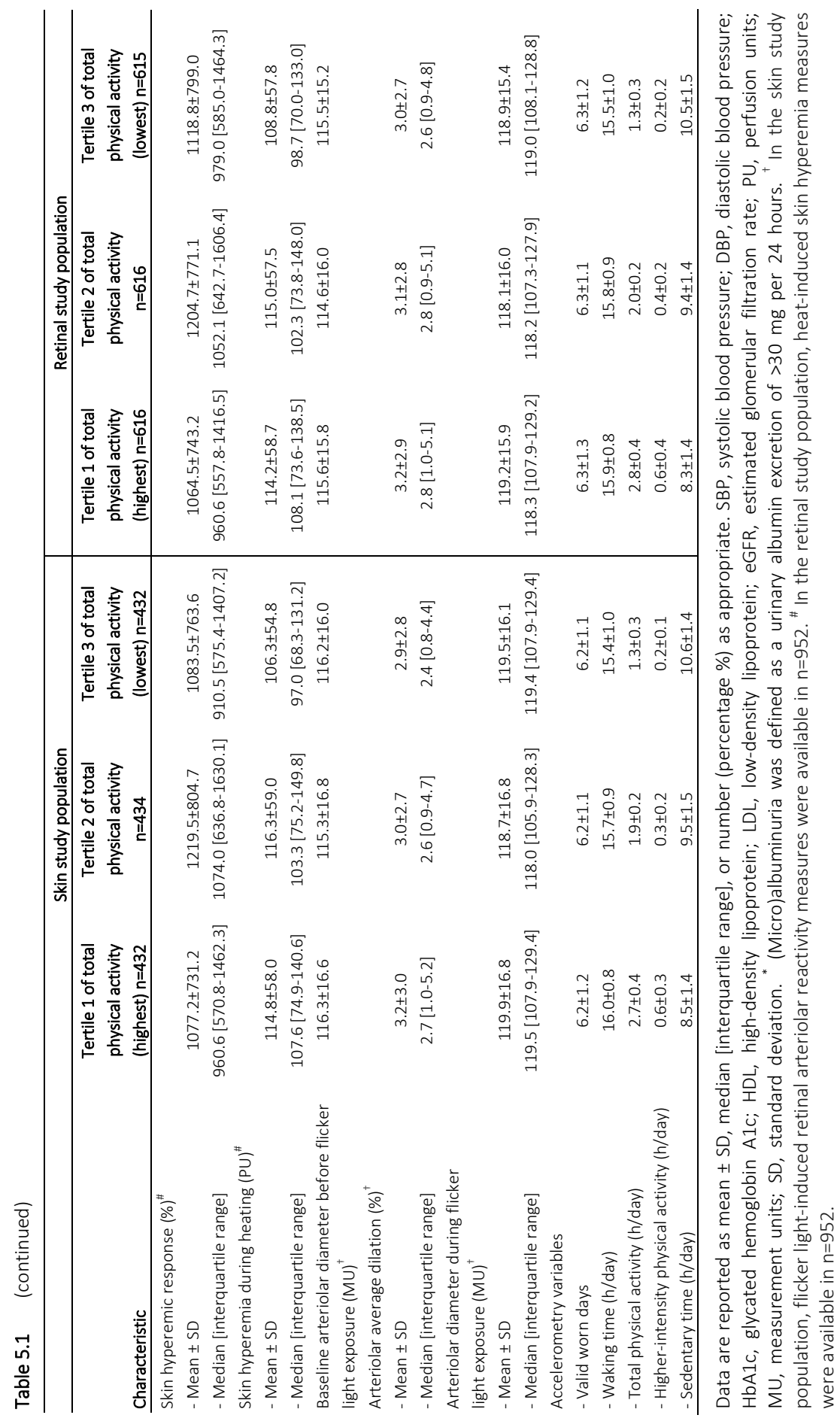




\section{Associations of total and higher-intensity physical activity with skin hyperemia and retinal arteriolar dilation}

In adjusted analyses, total physical activity and higher-intensity physical activity were not significantly associated with heat-induced skin hyperemia (Table 5.2). However, the associations of total physical activity and higher-intensity physical activity with heat-induced skin hyperemia differed between individuals without and with T2D $\left(P_{\text {interactions }}<0.10\right.$, Table 5.2). In individuals without T2D, total and higher-intensity physical activity were not significantly associated with heat-induced skin hyperemia. In contrast, in individuals with T2D, total and higher-intensity physical activity were directly associated with heat-induced skin hyperemia (regression coefficients per hour increase were $10 \mathrm{PU}(95 \% \mathrm{Cl}: 1 ; 18, \mathrm{P}=0.026)$ and $36 \mathrm{PU}(14 ; 58, \mathrm{P}=0.002)$, respectively (Table 5.2).

In adjusted analyses, total physical activity and higher-intensity physical activity were not significantly associated with flicker light-induced retinal arteriolar \%-dilation (Table 5.3). In addition, the associations of total physical activity and higher-intensity physical activity with retinal arteriolar \%-dilation did not differ significantly between individuals without and with $\mathrm{T} 2 \mathrm{D}$ ( $\mathrm{P}_{\text {interactions }}>0.10$, Table 5.3).

\section{Associations of sedentary behavior with skin hyperemia and retinal arteriolar dilation}

In adjusted analyses, total sedentary time was not associated with heat-induced skin hyperemia (Table 5.2). Although associations between total sedentary time and heatinduced skin hyperemia differed statistically between individuals without and with T2D $\left(P_{\text {interaction }}<0.10\right)$, stratified analyses in individuals without and with T2D did not show significant associations of total sedentary time and heat-induced skin hyperemia (Table 5.2).

In adjusted analyses, total sedentary time was not significantly associated with flicker light-induced retinal arteriolar \%-dilation (Table 5.3). In addition, the association between total sedentary time and retinal arteriolar \%-dilation did not differ significantly between individuals without and with T2D ( $P_{\text {interaction }}>0.10$, Table 5.3). 

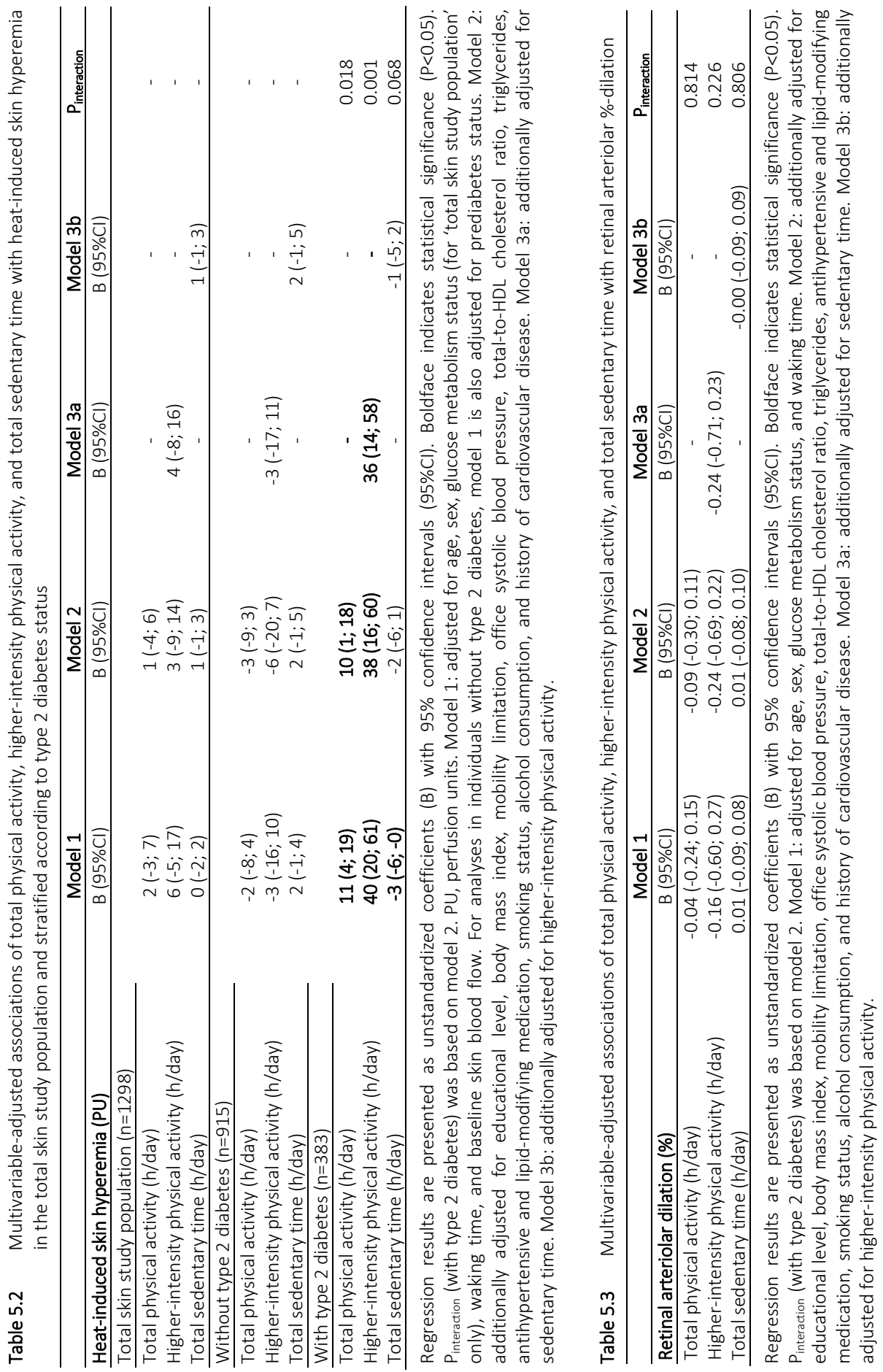


\section{Additional analyses}

As associations of total and higher-intensity physical activity with heat-induced skin hyperemia were stronger in individuals with as compared to those without T2D, we further explored interactions between continuous measures of glycemia (i.e. HbA1c, fasting plasma glucose, and 2-h postload glucose) and total and higher-intensity physical activity. Statistically significant interactions were found between 2-h postload glucose and total physical activity, and between $\mathrm{HbA1c}$, fasting plasma glucose, and 2-h postload glucose and higher-intensity physical activity (all $P_{\text {interactions }}<0.10$; data not shown). In individuals with prediabetes, regression coefficients of the associations of total and higher-intensity physical activity with skin hyperemia, were numerically in between those of individuals without and with T2D (Supplemental Table S5.5).

Qualitatively similar associations of total physical activity, higher-intensity physical activity, and total sedentary time with skin hyperemia and retinal arteriolar \%-dilation were observed in a range of additional analyses. First, when heat-induced increase in skin blood flow (in PU) from skin baseline or flicker light-induced increase (in MU) in retinal arteriolar diameter from baseline were used as outcomes (data not shown). Second, when substituting office systolic blood pressure for 24-h ambulatory systolic blood pressure (data not shown, for skin and retinal analyses, 24-h ambulatory blood pressure was available in $n=1151$ individuals ( $n=812$ without and $n=339$ with T2D) and $\mathrm{n}=1632$ ( $\mathrm{n}=1180$ without and $\mathrm{n}=452$ with T2D), respectively). Third, after additional adjustment for daily caloric intake (data not shown, for skin and retinal analyses, daily caloric intake was available in $n=1219$ individuals ( $n=863$ without and $n=356$ with T2D) and $n=1755$ ( $n=1276$ without and $n=479$ with T2D), respectively), or occupational status (data not shown, for skin and retinal analyses, occupational status was available in $n=1122$ individuals ( $n=807$ without and $n=315$ with T2D) and $n=1609(n=1178$ without and $n=431$ with T2D), respectively). Fourth, after additional adjustment for eGFR, urinary albumin excretion, and the presence of retinopathy (data not shown, for skin and retinal analyses, data on these additional covariates were available in $n=1163$ individuals ( $n=809$ without and $n=354$ with T2D) and $n=1774$ ( $n=1288$ without and $n=486$ with T2D), respectively). Fifth, when antihypertensive medication was further specified into reninangiotensin-aldosterone system (RAAS)-inhibiting (with or without other types of antihypertensives) and non-RAAS-inhibiting antihypertensives only (data not shown; RAAS-inhibiting antihypertensives included angiotensin-converting-enzyme inhibitors, angiotensin receptor blockers, and renin blockers). Sixth, after additional adjustment for time of the day or month of the year when the skin and retinal measurements were done (to adjust for possible diurnal or seasonal influences; data not shown). Seventh, associations of total physical activity, higher-intensity physical activity, and total sedentary time with skin hyperemia and retinal arteriolar \%-dilation did not differ 
between women and men (all $\mathrm{P}_{\text {interactions }}>0.10$ ). Last, collinearity diagnostics revealed no problematic multicollinearity in any of the analyses (i.e. all tolerance values $\geq 0.10$ and variance inflation factors $\leq 10$ ).

\section{Discussion}

In this population-based study, we tested the hypothesis that habitually low levels of physical activity and high levels of sedentary time (objectively quantified with an accelerometer) can affect microvascular endothelial function. There were three novel findings. First, higher levels of total and higher-intensity physical activity were independently associated with greater skin microvascular vasodilation in individuals with, but not in those without T2D. Second, and in contrast to our hypothesis, sedentary time was not associated with skin microvascular function. Third, physical activity and sedentary behavior were not statistically significantly associated with retinal microvascular function. These findings suggest that increasing habitual daily physical activity should be investigated as a means to improving microvascular function in T2D, with the ultimate goal of reducing risk of heart failure ${ }^{1}$, (lacunar) stroke ${ }^{2}$, depression ${ }^{3}$, cognitive decline ${ }^{4}$, retinopathy ${ }^{5}$, chronic kidney disease ${ }^{6}$, and neuropathy ${ }^{5}$.

Both heat-induced skin hyperemia and flicker light-induced retinal arteriolar dilation are likely a reflection of microvascular endothelial function, as they both depend on nitric oxide bioavailability ${ }^{51,52}$, possibly in conjunction with vascular smooth muscle cell function $^{53,54}$, and/or neuronal function ${ }^{55,56}$. Mechanistically, higher levels of physical activity and lower levels of sedentary time are thought to increase nitric oxide bioavailability ${ }^{29,30}$, presumably together with beneficial effects on low-grade inflammation ${ }^{31,32}$, both of which can improve microvascular endothelial function ${ }^{33}$.

The associations of higher levels of total and higher-intensity physical activity with greater heat-induced skin hyperemia in individuals with T2D are in agreement with earlier small studies ${ }^{57,58}$. Stronger associations in individuals with T2D, as compared to those without, can be explained by beneficial effects of physical activity on nitric oxide bioavailability ${ }^{30,58}$. These effects are likely more prominent in individuals with hyperglycemia, as hyperglycemia is a potent inducer of impaired nitric oxide bioavailability $^{34}$ and microvascular (endothelial) dysfunction ${ }^{35}$. A close relationship between hyperglycemia and the strength of these associations is supported by statistically significant interactions between measures of glycemia (including prediabetes (Supplemental Table S5.5)) and habitual total and higher-intensity physical activity.

The absence of an association between higher levels of habitual total and higherintensity physical activity and greater skin microvascular function in individuals without 
T2D contrasts with earlier studies which showed beneficial effects of (chronic) intensive exercise on skin microvascular function in healthy individuals ${ }^{20,21,59}$. A possible explanation is that in individuals without T2D, physical activity-induced improvement in skin microvascular function is observed only at higher intensities and longer duration of physical activity than those in our study ${ }^{20}$.

The absence of an inverse association between higher levels of habitual total sedentary time and skin microvascular function contrasts with earlier studies with uninterrupted sedentary time protocols ${ }^{19,25,26}$. Mechanistically, sedentary behavior is thought to reduce shear stress, leading to less nitric oxide synthesis and reduced endothelium-dependent vasodilation ${ }^{29}$. Insufficient duration of total sedentary time (mean \pm SD: $9.5 \pm 1.7 \mathrm{~h} /$ day; range: 2.5 to $15.9 \mathrm{~h} /$ day) is unlikely to explain the absence of an inverse association between sedentary behavior and microvascular function. A likelier explanation is that frequent transient interruptions of sedentary behavior by standing and/or walking can restore sedentary behavior-induced microvascular dysfunction ${ }^{19,25}$, presumably via changes in shear stress ${ }^{60}$. Indeed, a higher number of such interruptions, which in our study population occurred on average $\sim 4$ times per sedentary hour, have been shown to be associated with reduced cardiovascular mortality ${ }^{61}$.

Autoregulation of retinal blood flow can explain why physical activity and sedentary behavior were not associated with retinal microvascular function ${ }^{39,40,62}$. During physical activity, constriction of small retinal arterioles increases retinal vascular resistance in proportion to the increase in ocular perfusion pressure ${ }^{40}$ to stabilize retinal perfusion $^{39,40}$, presumably to maintain visual acuity ${ }^{41}$. Our finding of a numerically lower baseline retinal arteriolar diameter with increased levels of higher-intensity physical activity (Supplemental Table S5.4) is consistent with this interpretation. Whether chronic habitual sedentary behavior alters ocular blood flow is currently unknown, but unlikely, as retinal autoregulation has been shown to preserve stable retinal perfusion during transient sedentary time at different postures (e.g. sitting and lying) ${ }^{62}$.

Strengths of our study include its size and population-based design; the use of a waterproof-attached accelerometer, which has resulted in improved wear time compliance $^{63}$, and measures which are more precise and valid than when selfreported $^{28}$; the extensive assessment of, and adjustment for, potential confounders; the use of two independent methods to directly assess microvascular function in different microvascular beds; and the broad array of additional analyses, which all gave consistent results.

Our study had some limitations. First, the data were cross-sectional. Therefore, we cannot exclude reverse causality, i.e. that microvascular dysfunction leads to suboptimal delivery of oxygen and nutrients to tissues (e.g. muscle) on demand, and therefore may hamper physical activity. Second, total and higher-intensity physical activity and total 
sedentary time were measured during one week, which may not truly reflect habitual behavior. However, with an average wear time of 6.2 days in our study, compliance to the 8 days wear time protocol was good, and sufficient to reliably estimate habitual sedentary behavior and close to optimal wear time for estimation of habitual physical activity $^{64}$. Third, higher-intensity physical activity was based on step frequency, which is less precise than acceleration-based determination. However, we used a cut-off point of $>110$ steps/minute for higher-intensity physical activity, which roughly equals a Metabolic Equivalent of Task (MET)-score of $\geq 3.0$ (which indicates moderate-to-vigorous intensity activity ${ }^{47}$. Last, although we adjusted for many potential confounders, we cannot fully exclude residual confounding by variables not included in these analyses (e.g. dietary habits).

This large population-based study, with postured-based accelerometry data, showed that higher levels of total and higher-intensity physical activity were independently associated with greater skin microvascular vasodilation in individuals with, but not in those without T2D. This is consistent with beneficial effects of physical activity on nitric oxide bioavailability, which are likely more prominent in individuals with hyperglycemia. These findings suggest that increasing habitual daily physical activity should be investigated as a means to improving microvascular function in $\mathrm{T} 2 \mathrm{D}$, with the ultimate goal of reducing risk of heart failure ${ }^{1}$, (lacunar) stroke ${ }^{2}$, depression ${ }^{3}$, cognitive decline ${ }^{4}$, retinopathy ${ }^{5}$, chronic kidney disease ${ }^{6}$, and neuropathy ${ }^{5}$. 


\section{References}

1. Lee JF, Barrett-O'Keefe Z, Garten RS, Nelson AD, Ryan JJ, Nativi JN, Richardson RS and Wray DW. Evidence of microvascular dysfunction in heart failure with preserved ejection fraction. Heart. 2016;102:278-84.

2. Knottnerus IL, Ten Cate H, Lodder J, Kessels F and van Oostenbrugge RJ. Endothelial dysfunction in lacunar stroke: a systematic review. Cerebrovasc Dis. 2009;27:519-26.

3. Santos M, Xekardaki A, Kovari E, Gold G, Bouras C and Giannakopoulos P. Microvascular pathology in late-life depression. J Neurol Sci. 2012;322:46-9.

4. De Silva TM and Faraci FM. Microvascular Dysfunction and Cognitive Impairment. Cell Mol Neurobiol. 2016;36:241-58.

5. Gupta A and Bhatnagar S. Vasoregression: A Shared Vascular Pathology Underlying Macrovascular And Microvascular Pathologies? OMICS. 2015;19:733-53.

6. Zafrani L and Ince C. Microcirculation in Acute and Chronic Kidney Diseases. Am J Kidney Dis. 2015;66:1083-94.

7. Stratton IM, Adler AI, Neil HA, Matthews DR, Manley SE, Cull CA, Hadden D, Turner RC and Holman RR. Association of glycaemia with macrovascular and microvascular complications of type 2 diabetes (UKPDS 35): prospective observational study. BMJ. 2000;321:405-12.

8. Tremblay MS, Aubert S, Barnes JD, Saunders TJ, Carson V, Latimer-Cheung AE, Chastin SF, Altenburg TM, Chinapaw MJ and Participants STCP. Sedentary Behavior Research Network (SBRN) - Terminology Consensus Project process and outcome. Int J Behav Nutr Phys Act. 2017;14:75.

9. Lee IM, Shiroma EJ, Lobelo F, Puska P, Blair SN, Katzmarzyk PT and Lancet Physical Activity Series Working G. Effect of physical inactivity on major non-communicable diseases worldwide: an analysis of burden of disease and life expectancy. Lancet. 2012;380:219-29.

10. Chen SM, Tsai TH, Hang CL, Yip HK, Fang CY, Wu CJ and Guo GB. Endothelial dysfunction in young patients with acute ST-elevation myocardial infarction. Heart Vessels. 2011;26:2-9.

11. Witte DR, Westerink J, de Koning EJ, van der Graaf Y, Grobbee DE and Bots ML. Is the association between flow-mediated dilation and cardiovascular risk limited to low-risk populations? J Am Coll Cardiol. 2005;45:1987-93.

12. Young DR, Reynolds K, Sidell M, Brar S, Ghai NR, Sternfeld B, Jacobsen SJ, Slezak JM, Caan B and Quinn V. Effects of physical activity and sedentary time on the risk of heart failure. Circ Heart Fail. 2014;7:21-7.

13. Willey JZ, Moon YP, Sacco RL, Greenlee H, Diaz KM, Wright CB, Elkind MS and Cheung YK. Physical inactivity is a strong risk factor for stroke in the oldest old: Findings from a multi-ethnic population (the Northern Manhattan Study). Int J Stroke. 2017;12:197-200.

14. Schuch F, Vancampfort D, Firth J, Rosenbaum S, Ward P, Reichert T, Bagatini NC, Bgeginski R and Stubbs B. Physical activity and sedentary behavior in people with major depressive disorder: A systematic review and meta-analysis. J Affect Disord. 2017;210:139-50.

15. Steinberg SI, Sammel MD, Harel BT, Schembri A, Policastro C, Bogner HR, Negash S and Arnold SE. Exercise, sedentary pastimes, and cognitive performance in healthy older adults. Am J Alzheimers Dis Other Demen. 2015;30:290-8.

16. Praidou A, Harris M, Niakas D and Labiris G. Physical activity and its correlation to diabetic retinopathy. J Diabetes Complications. 2017;31:456-61.

17. White SL, Dunstan DW, Polkinghorne KR, Atkins RC, Cass A and Chadban SJ. Physical inactivity and chronic kidney disease in Australian adults: the AusDiab study. Nutr Metab Cardiovasc Dis. 2011;21:104-12.

18. Singleton JR, Smith AG and Marcus RL. Exercise as Therapy for Diabetic and Prediabetic Neuropathy. Curr Diab Rep. 2015;15:120.

19. Restaino RM, Holwerda SW, Credeur DP, Fadel PJ and Padilla J. Impact of prolonged sitting on lower and upper limb micro- and macrovascular dilator function. Exp Physiol. 2015;100:829-38.

20. Tew GA, George KP, Cable NT and Hodges GJ. Endurance exercise training enhances cutaneous microvascular reactivity in post-menopausal women. Microvasc Res. 2012;83:223-8.

21. Roche DM, Rowland TW, Garrard M, Marwood S and Unnithan VB. Skin microvascular reactivity in trained adolescents. Eur J Appl Physiol. 2010;108:1201-8. 
22. Wang JS. Effects of exercise training and detraining on cutaneous microvascular function in man: the regulatory role of endothelium-dependent dilation in skin vasculature. Eur J Appl Physiol. 2005;93: 429-34.

23. Navasiolava NM, Dignat-George F, Sabatier F, Larina IM, Demiot C, Fortrat JO, Gauquelin-Koch G, Kozlovskaya IB and Custaud MA. Enforced physical inactivity increases endothelial microparticle levels in healthy volunteers. Am J Physiol Heart Circ Physiol. 2010;299:H248-56.

24. Vranish JR, Young BE, Kaur J, Patik JC, Padilla J and Fadel PJ. Influence of Sex on Microvascular and Macrovascular Responses to Prolonged Sitting. Am J Physiol Heart Circ Physiol. 2017;312:H800-5.

25. Demiot C, Dignat-George F, Fortrat JO, Sabatier F, Gharib C, Larina I, Gauquelin-Koch G, Hughson R and Custaud MA. WISE 2005: chronic bed rest impairs microcirculatory endothelium in women. Am J Physiol Heart Circ Physiol. 2007;293:H3159-64.

26. Hamburg NM, McMackin CJ, Huang AL, Shenouda SM, Widlansky ME, Schulz E, Gokce N, Ruderman NB, Keaney JF, Jr. and Vita JA. Physical inactivity rapidly induces insulin resistance and microvascular dysfunction in healthy volunteers. Arterioscler Thromb Vasc Biol. 2007;27:2650-6.

27. Anuradha S, Dunstan DW, Healy GN, Shaw JE, Zimmet PZ, Wong TY and Owen N. Physical activity, television viewing time, and retinal vascular caliber. Med Sci Sports Exerc. 2011;43:280-6.

28. Downs A, Van Hoomissen J, Lafrenz A and Julka DL. Accelerometer-measured versus self-reported physical activity in college students: implications for research and practice. I Am Coll Health. 2014;62:204-12.

29. Thosar SS, Johnson BD, Johnston JD and Wallace JP. Sitting and endothelial dysfunction: the role of shear stress. Med Sci Monit. 2012;18:RA173-80.

30. Nyberg M, Blackwell JR, Damsgaard R, Jones AM, Hellsten Y and Mortensen SP. Lifelong physical activity prevents an age-related reduction in arterial and skeletal muscle nitric oxide bioavailability in humans. J Physiol. 2012;590:5361-70.

31. Lambert CP, Wright NR, Finck BN and Villareal DT. Exercise but not diet-induced weight loss decreases skeletal muscle inflammatory gene expression in frail obese elderly persons. J Appl Physiol (1985). 2008;105:473-8.

32. Parsons TJ, Sartini C, Welsh P, Sattar N, Ash S, Lennon LT, Wannamethee SG, Lee IM, Whincup PH and Jefferis BJ. Physical Activity, Sedentary Behavior, and Inflammatory and Hemostatic Markers in Men. Med Sci Sports Exerc. 2017;49:459-65.

33. Matlung HL, Bakker EN and VanBavel E. Shear stress, reactive oxygen species, and arterial structure and function. Antioxid Redox Signal. 2009;11:1699-709.

34. Hamed S, Brenner B and Roguin A. Nitric oxide: a key factor behind the dysfunctionality of endothelial progenitor cells in diabetes mellitus type-2. Cardiovasc Res. 2011;91:9-15.

35. Sörensen BM, Houben AJ, Berendschot TT, Schouten JS, Kroon AA, van der Kallen CJ, Henry RM, Koster A, Reesink KD, Dagnelie PC, Schaper NC, Schalkwijk CG, Schram MT and Stehouwer CD. Hyperglycemia Is the Main Mediator of Prediabetes- and Type 2 Diabetes-Associated Impairment of Microvascular Function: The Maastricht Study. Diabetes Care. 2017;40:e103-5.

36. Nguyen TT, Kreis AJ, Kawasaki R, Wang JJ, Seifert BU, Vilser W, Nagel E and Wong TY. Reproducibility of the retinal vascular response to flicker light in Asians. Curr Eye Res. 2009;34:1082-8.

37. Agarwal SC, Allen J, Murray A and Purcell IF. Comparative reproducibility of dermal microvascular blood flow changes in response to acetylcholine iontophoresis, hyperthermia and reactive hyperaemia. Physiol Meas. 2010;31:1-11.

38. Kenny GP and McGinn R. Restoration of thermoregulation after exercise. J Appl Physiol (1985). 2017;122:933-44.

39. Hayashi N, Ikemura T and Someya N. Effects of dynamic exercise and its intensity on ocular blood flow in humans. Eur J Appl Physiol. 2011;111:2601-6.

40. Risner D, Ehrlich R, Kheradiya NS, Siesky B, McCranor L and Harris A. Effects of exercise on intraocular pressure and ocular blood flow: a review. J Glaucoma. 2009;18:429-36.

41. Hayashi N, Someya N and Ikemura T. Effects of hyper- and hypocapnea on choroidal and retinal blood flows and the visual acuity. Faseb Journal. 2010;24.

42. Schram MT, Sep SJ, van der Kallen CJ, Dagnelie PC, Koster A, Schaper NC, Henry RM and Stehouwer CD. The Maastricht Study: an extensive phenotyping study on determinants of type 2 diabetes, its complications and its comorbidities. Eur J Epidemiol. 2014;29:439-51. 
43. Sörensen BM, Houben AJ, Berendschot TT, Schouten JS, Kroon AA, van der Kallen CJ, Henry RM, Koster A, Sep SJ, Dagnelie PC, Schaper NC, Schram MT and Stehouwer CD. Prediabetes and Type 2 Diabetes Are Associated With Generalized Microvascular Dysfunction: The Maastricht Study. Circulation. 2016;134:1339-52.

44. Vickers AJ. The use of percentage change from baseline as an outcome in a controlled trial is statistically inefficient: a simulation study. BMC Med Res Methodol. 2001;1:6.

45. Atkinson $\mathrm{G}$ and Batterham AM. The percentage flow-mediated dilation index: a large-sample investigation of its appropriateness, potential for bias and causal nexus in vascular medicine. Vasc Med. 2013;18:354-65.

46. van der Berg JD, Willems PJ, van der Velde JH, Savelberg HH, Schaper NC, Schram MT, Sep SJ, Dagnelie PC, Bosma H, Stehouwer CD and Koster A. Identifying waking time in 24-h accelerometry data in adults using an automated algorithm. J Sports Sci. 2016;34:1867-73.

47. Tudor-Locke C, Craig CL, Brown WJ, Clemes SA, De Cocker K, Giles-Corti B, Hatano Y, Inoue S, Matsudo SM, Mutrie N, Oppert JM, Rowe DA, Schmidt MD, Schofield GM, Spence JC, Teixeira PJ, Tully MA and Blair SN. How many steps/day are enough? For adults. Int J Behav Nutr Phys Act. 2011;8:79.

48. Tudor-Locke $C$ and Rowe DA. Using cadence to study free-living ambulatory behaviour. Sports Med. 2012;42:381-98.

49. Tremblay MS, Colley RC, Saunders TJ, Healy GN and Owen N. Physiological and health implications of a sedentary lifestyle. Appl Physiol Nutr Metab. 2010;35:725-40.

50. Martens RJ, Henry RM, Houben AJ, van der Kallen CJ, Kroon AA, Schalkwijk CG, Schram MT, Sep SJ, Schaper NC, Dagnelie PC, Muris DM, Gronenschild EH, van der Sande FM, Leunissen KM, Kooman JP and Stehouwer CD. Capillary Rarefaction Associates with Albuminuria: The Maastricht Study. J Am Soc Nephrol. 2016;27:3748-57.

51. Kellogg DL, Jr., Liu Y, Kosiba IF and O'Donnell D. Role of nitric oxide in the vascular effects of local warming of the skin in humans. J Appl Physiol (1985). 1999;86:1185-90.

52. Dorner GT, Garhofer G, Kiss B, Polska E, Polak K, Riva CE and Schmetterer L. Nitric oxide regulates retinal vascular tone in humans. Am J Physiol Heart Circ Physiol. 2003;285:H631-6.

53. Montero D, Pierce GL, Stehouwer CD, Padilla J and Thijssen DH. The impact of age on vascular smooth muscle function in humans. J Hypertens. 2015;33:445-53.

54. Lacolley P, Regnault V, Nicoletti A, Li Z and Michel JB. The vascular smooth muscle cell in arterial pathology: a cell that can take on multiple roles. Cardiovasc Res. 2012;95:194-204.

55. Minson CT, Berry LT and Joyner MJ. Nitric oxide and neurally mediated regulation of skin blood flow during local heating. J Appl Physiol (1985). 2001;91:1619-26.

56. Falsini B, Riva CE and Logean E. Flicker-evoked changes in human optic nerve blood flow: relationship with retinal neural activity. Invest Ophthalmol Vis Sci. 2002;43:2309-16.

57. Colberg SR, Parson HK, Holton DR, Nunnold T and Vinik Al. Cutaneous blood flow in type 2 diabetic individuals after an acute bout of maximal exercise. Diabetes Care. 2003;26:1883-8.

58. Colberg SR, Stansberry KB, McNitt PM and Vinik Al. Chronic exercise is associated with enhanced cutaneous blood flow in type 2 diabetes. J Diabetes Complications. 2002;16:139-45.

59. Franzoni F, Galetta F, Morizzo C, Lubrano V, Palombo C, Santoro G, Ferrannini E and Quinones-Galvan A. Effects of age and physical fitness on microcirculatory function. Clin Sci. 2004;106:329-35.

60. Tinken TM, Thijssen DH, Hopkins N, Black MA, Dawson EA, Minson CT, Newcomer SC, Laughlin MH, Cable NT and Green DJ. Impact of shear rate modulation on vascular function in humans. Hypertension. 2009;54:278-85.

61. Diaz KM, Howard VJ, Hutto B, Colabianchi N, Vena JE, Safford MM, Blair SN and Hooker SP. Patterns of Sedentary Behavior and Mortality in U.S. Middle-Aged and Older Adults: A National Cohort Study. Ann Intern Med. 2017.

62. Feke GT and Pasquale LR. Retinal blood flow response to posture change in glaucoma patients compared with healthy subjects. Ophthalmology. 2008;115:246-52.

63. Tudor-Locke C, Barreira TV, Schuna JM, Jr., Mire EF, Chaput JP, Fogelholm M, Hu G, Kuriyan R, Kurpad A, Lambert EV, Maher C, Maia J, Matsudo V, Olds T, Onywera V, Sarmiento OL, Standage M, Tremblay MS, Zhao P, Church TS, Katzmarzyk PT and Group IR. Improving wear time compliance with a 24-hour waist-worn accelerometer protocol in the International Study of Childhood Obesity, Lifestyle and the Environment (ISCOLE). Int J Behav Nutr Phys Act. 2015;12:11. 
Chapter 5

64. Barreira TV, Hamilton MT, Craft LL, Gapstur SM, Siddique J and Zderic TW. Intra-individual and interindividual variability in daily sitting time and MVPA. J Sci Med Sport. 2016;19:476-81. 


\section{Supplemental Material to chapter 5}

\section{Supplemental Methods}

\section{Assessment of retinal arteriolar microvascular function ${ }^{1}$}

The retinal arteriolar dilation response to flicker light was measured in a dimly lit room by use of the Dynamic Vessel Analyzer (DVA) (Imedos, Jena, Germany). For safety reasons, participants with an intraocular pressure exceeding $30 \mathrm{mmHg}$ were excluded from retinal measurements. Per participant either the left or right eye was selected depending on the time of day the measurement was performed and without reference to participant characteristics.

During the measurement, the participant was instructed and encouraged to focus on the tip of a fixated needle inside the retinal camera (FF450; Carl Zeiss GmbH, Jena, Germany), while the fundus of the eye was examined under green measuring light (530-600 nm, illumination of fundus approximately 6500 lux). A straight arteriolar segment of approximately $1.5 \mathrm{~mm}$ in length located 0.5 to 2.0 disc diameter from the margin of the optic disc in the temporal section was examined. When the specific vessel profile was recognized, vessel diameter was automatically and continuously measured for 150 seconds. A baseline recording of 50 seconds was followed by a 40-second flicker light exposure period (flicker frequency $12.5 \mathrm{~Hz}$, bright-to-dark contrast ratio 25:1) followed by a 60-second recovery period. The DVA automatically corrected for alterations in luminance caused by, for example, slight eye movements. During blinks and small eye movements, the registration stopped and restarted once the vessel segment was automatically re-identified.

\section{Assessment of patterns of physical activity and sedentary behavior}

Daily activity levels and patterns were measured using the activPAL3 ${ }^{\text {TM }}$ physical activity monitor (PAL technologies, Glasgow, UK) as has been described previously ${ }^{2}$. The activPAL3 ${ }^{\text {TM }}$ is a small $(53 \times 35 \times 7 \mathrm{~mm})$, lightweight (15 gram) triaxial accelerometer that records movement in the vertical, anteroposterior, and mediolateral axes, and also determines posture (sitting or lying, standing, and stepping) based on acceleration information. The device was attached directly to the skin on the front of the right thigh with transparent $3 \mathrm{M}$ Tegaderm ${ }^{\mathrm{TM}}$ tape, after the device had been waterproofed using a nitrile sleeve. Participants were asked to wear the accelerometer for 8 consecutive days, without removing the device at any time. To avoid inaccurately identifying of non-wear time, participants were asked not to replace the device once removed. Data were uploaded using the activePAL software and processed using customized software written in MATLAB R2013b (MathWorks, Natick, USA). Data from the first day were excluded 
from the analysis because participants performed physical function tests at the research center after the device was attached. In addition, data from the final wear day providing $\leq 14$ waking hours of data were excluded from the analysis. Participants were included if they provided at least 1 valid day ( $\geq 10$ hours of waking data).

\section{Detailed assessment of covariates}

Level of education was categorized into low (none, primary, or lower vocational education only), medium (intermediate general secondary, intermediate vocational, or higher general secondary education), and high (higher vocational education or university level of education). Alcohol consumption was categorized into non-consumers, low consumers ( $\leq 7$ glasses per week for women and $\leq 14$ glasses per week for men), and high consumers ( $>7$ glasses per week for women and $>14$ glasses per week for men). History of cardiovascular disease was assessed by web-based questionnaires and was defined as a history of myocardial infarction, stroke, or vascular surgery (including angioplasty) of coronary, carotid, abdominal aortic, or peripheral arteries. Occupational status was assess by questionnaire, and classified as self-employed, working for the government, salaried worker, disabled, rentier, retired, homemaker, unemployed, or other. Those who selected the option disabled, rentier, retired, homemaker, or unemployed were included in the non-employment group. Those in the class "other" were excluded, as their employment status could not be confirmed. Those who reported being self-employed, salaried worker, or working for the government were further classified as employed, and their occupational status was categorized according to the question "What category was your job?" as low (including option such as; without a profession, unschooled, schooled, and lower employee), intermediate or high employee, or self-employed.

\section{Statistical analysis}

Differences in general characteristics between individuals in the study populations and individuals excluded due to missing values were compared by Analyses of Variance (ANOVA) for continuous variables and $\chi^{2}$-test for categorical variables. 


\section{Supplemental Results}

\section{Associations of total and higher-intensity physical activity and total sedentary time with} baseline skin blood flow and baseline retinal arteriolar diameter

In adjusted analyses, total physical activity and higher-intensity physical activity were significantly associated with baseline skin blood flow (regression coefficients per hour increase were $0.89 \mathrm{PU}(95 \% \mathrm{Cl}$ : 0.32; 1.46, P=0.002) and 2.14 PU (0.82; 3.46, P=0.002), respectively). These associations did not differ significantly between individuals without and with type 2 diabetes (T2D) ( $P_{\text {interactions }}>0.10$, Supplemental Table S5.3). Total sedentary time was associated with baseline skin blood flow (regression coefficient per hour increase was $-0.24 \mathrm{PU}(-0.48 ;-0.00, \mathrm{P}=0.047))$. In addition, this association was significantly different between individuals without and with T2D ( $\left.P_{\text {interaction }}<0.10\right)$. In individuals without T2D total sedentary time was significantly associated with baseline skin blood flow (regression coefficient per hour increase was $-0.45 \mathrm{PU}(-0.74 ;-0.16$, $\mathrm{P}=0.003))$, whereas in individuals with T2D it was not; $0.26 \mathrm{PU}(-0.14 ; 0.66, \mathrm{P}=0.198$, Supplemental Table S5.3).

In adjusted analyses, total physical activity, higher-intensity physical activity, and total sedentary time were not significantly associated with baseline retinal arteriolar diameter (Supplemental Table S5.4). In addition, the associations of total physical activity, higher-intensity physical activity, and total sedentary time with baseline retinal arteriolar diameter did not differ significantly between individuals without and with T2D $\left(P_{\text {interactions }}>0.10\right.$, Supplemental Table S5.4).

\section{Supplemental Tables}




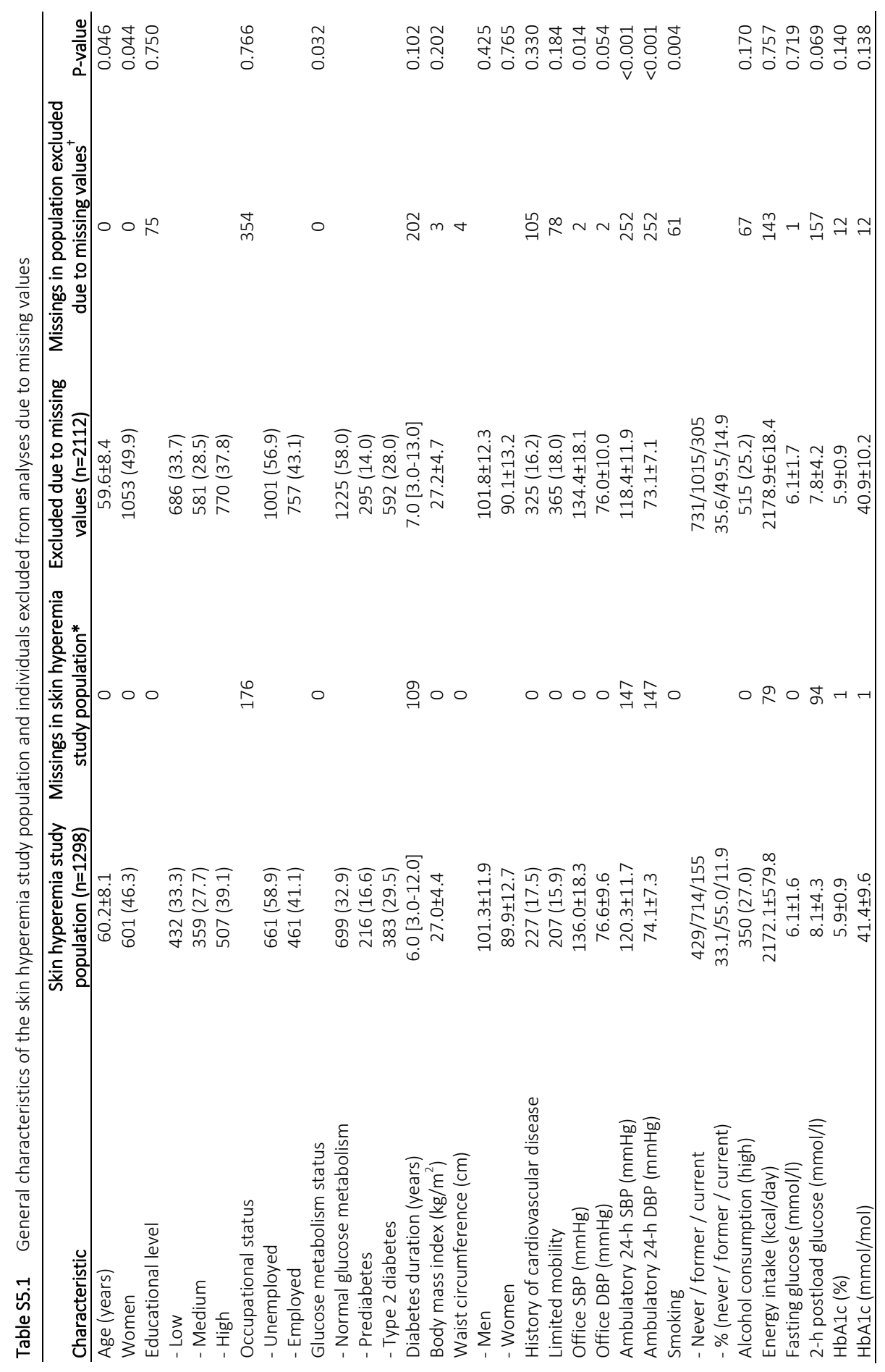




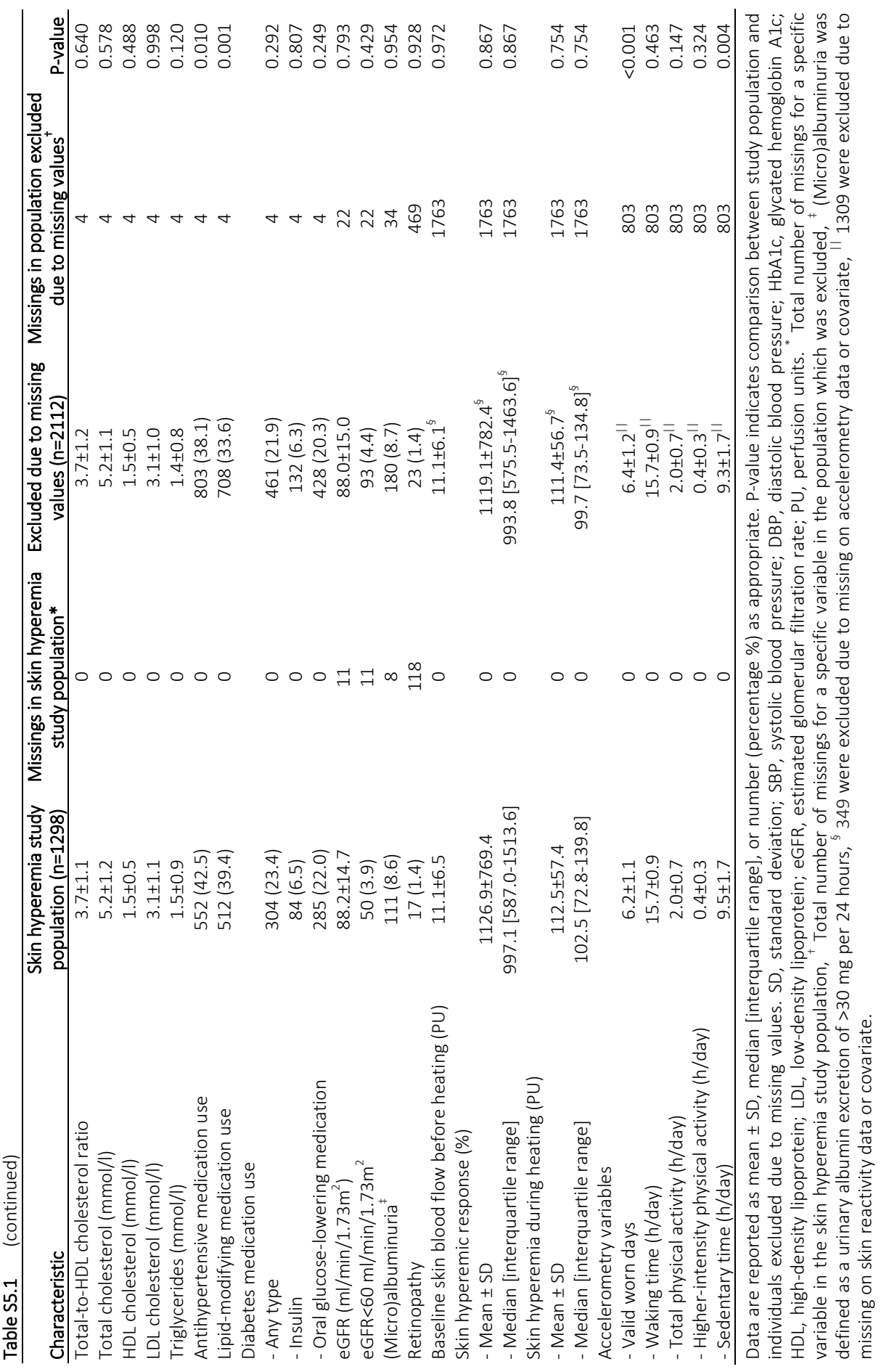




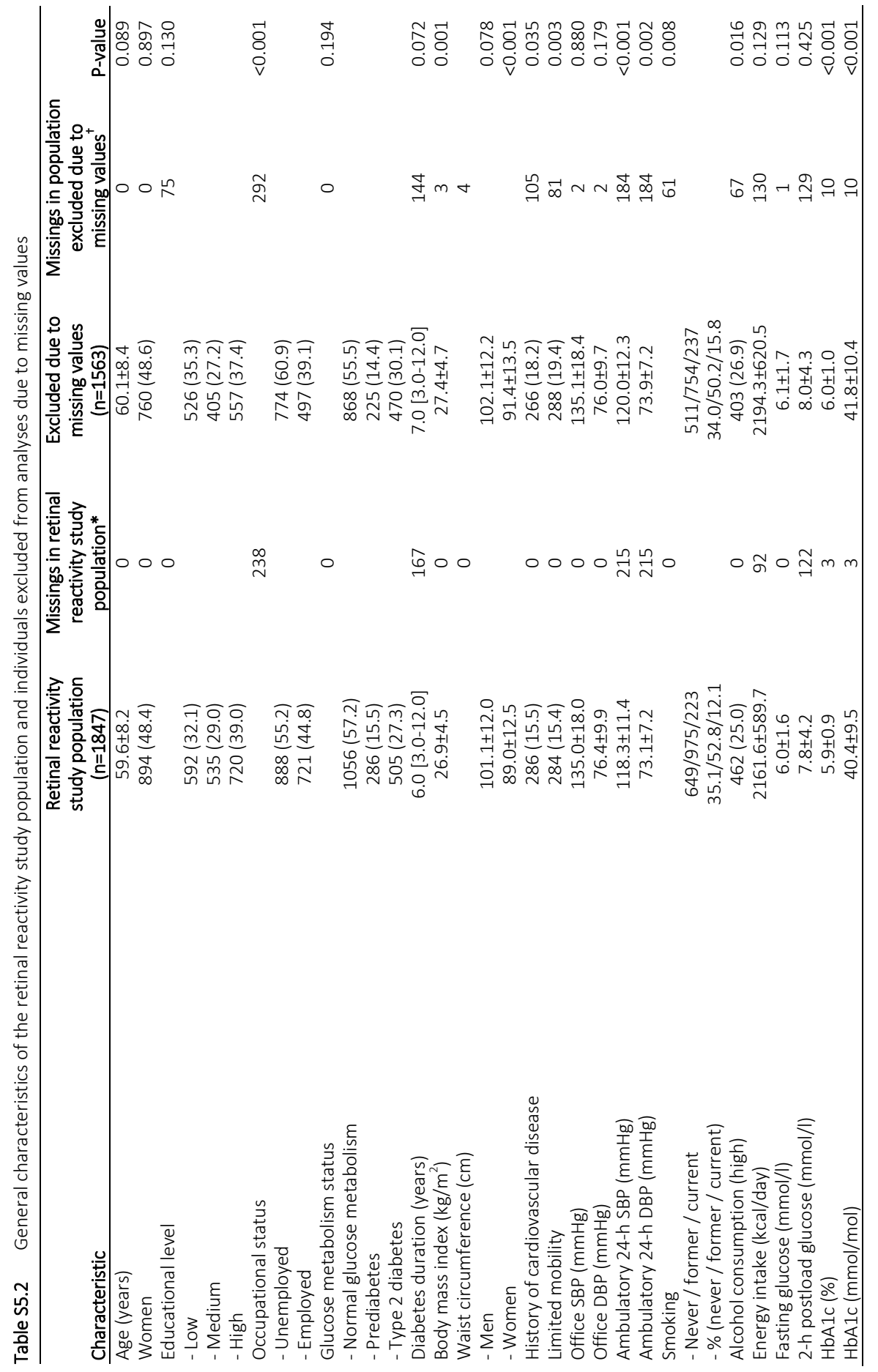




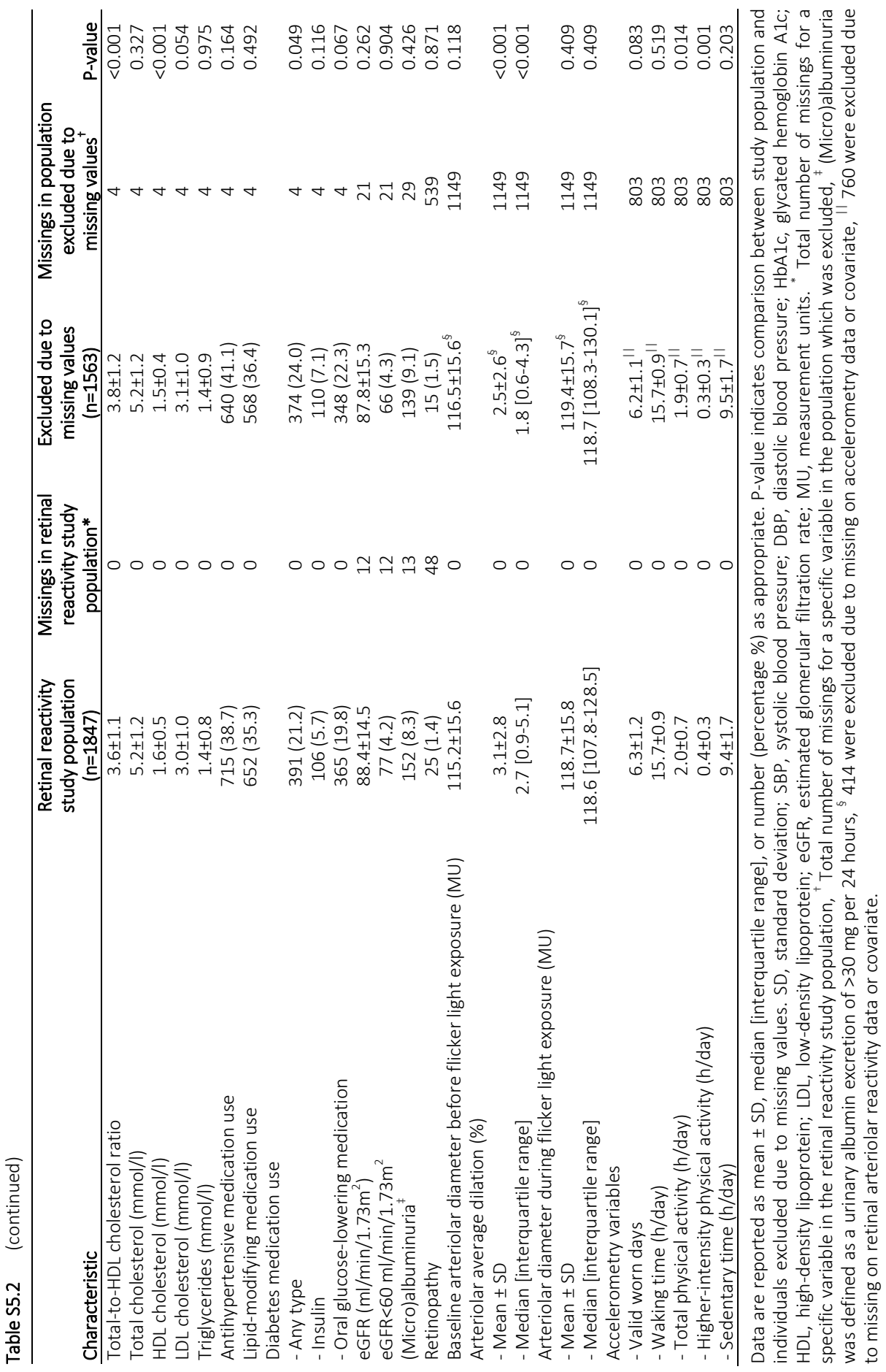




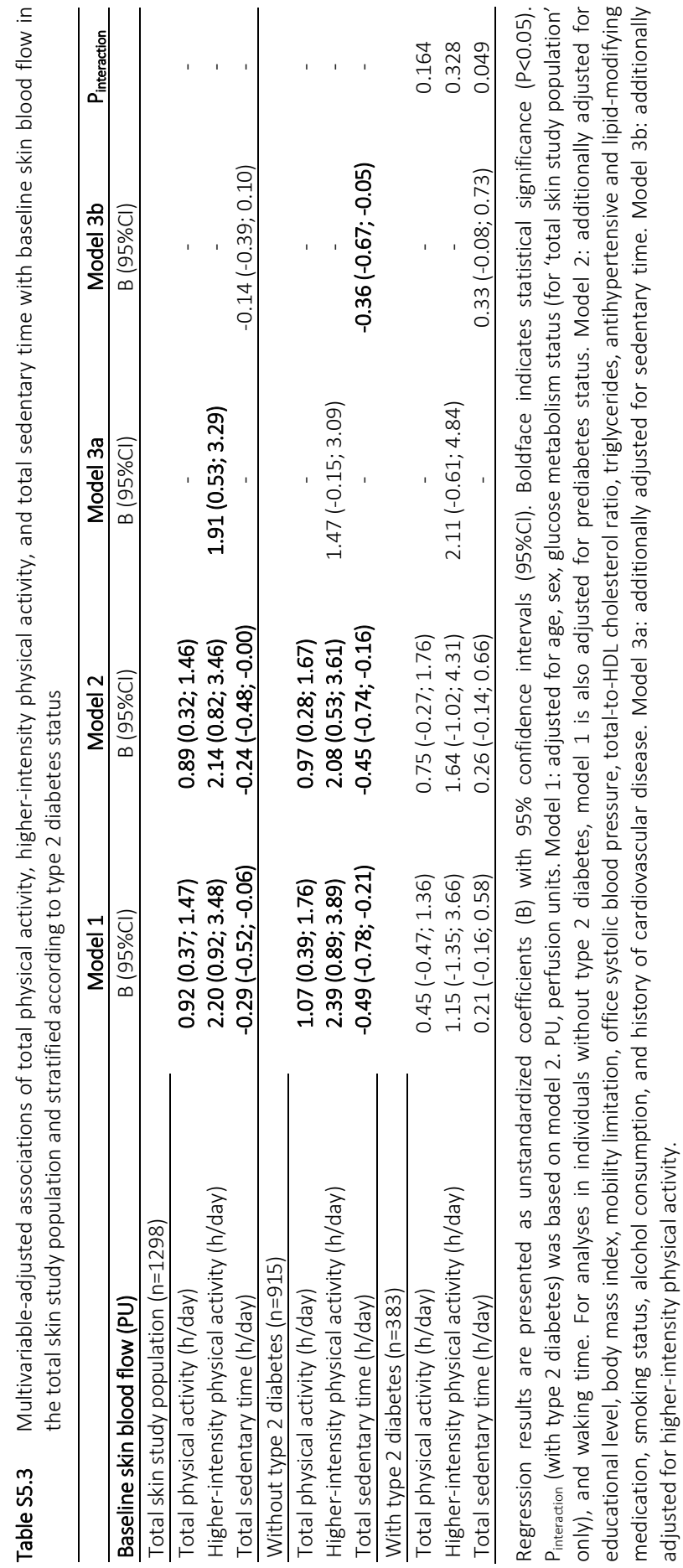


Physical activity and sedentary behavior versus microvascular function
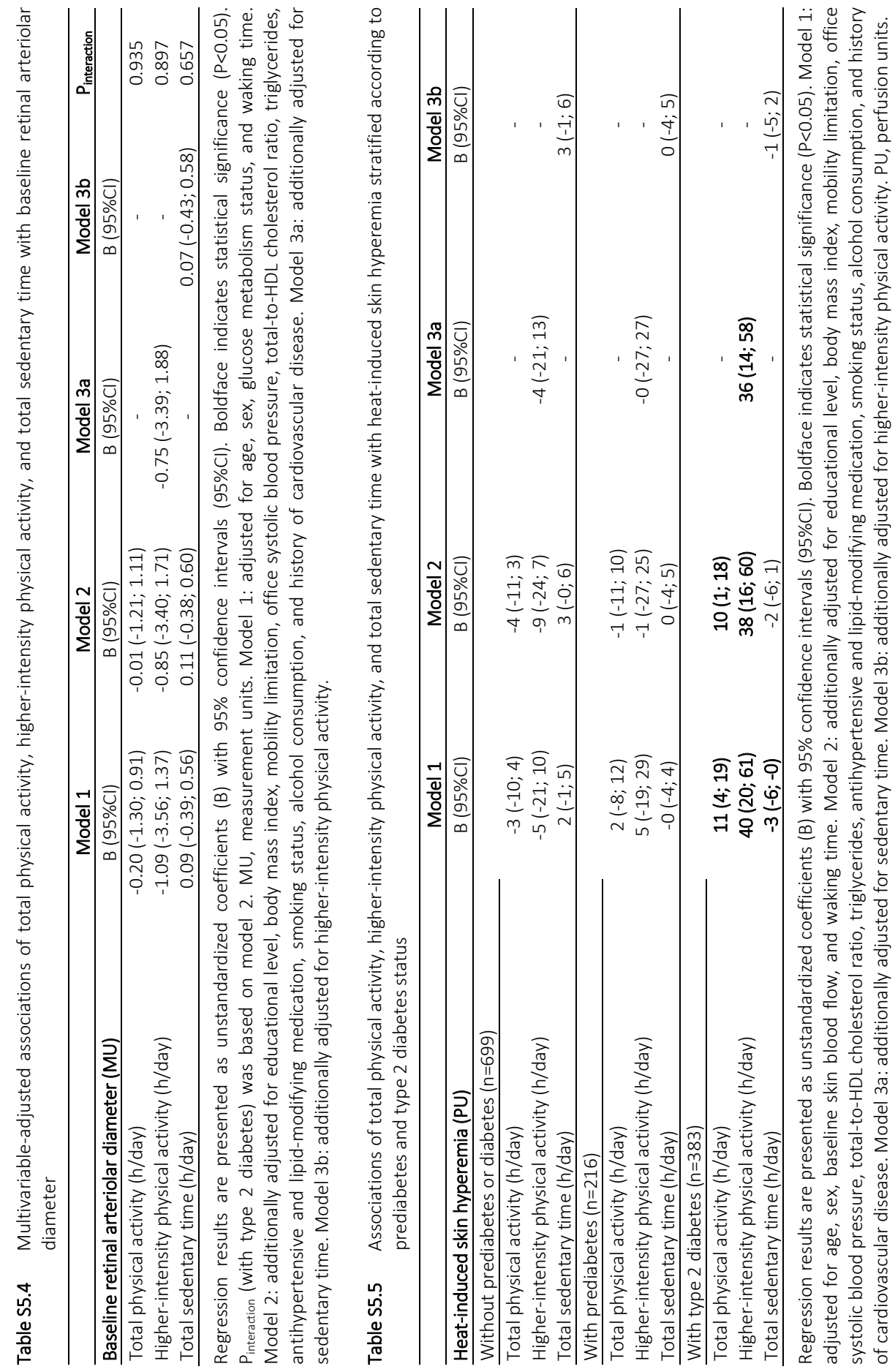


\section{References}

1. Sörensen BM, Houben AJ, Berendschot TT, Schouten JS, Kroon AA, van der Kallen CJ, Henry RM, Koster A, Sep SJ, Dagnelie PC, Schaper NC, Schram MT and Stehouwer CD. Prediabetes and Type 2 Diabetes Are Associated With Generalized Microvascular Dysfunction: The Maastricht Study. Circulation. 2016;134:1339-52.

2. van der Berg JD, Willems PJ, van der Velde JH, Savelberg HH, Schaper NC, Schram MT, Sep SJ, Dagnelie PC, Bosma H, Stehouwer CD and Koster A. Identifying waking time in 24-h accelerometry data in adults using an automated algorithm. J Sports Sci. 2016;34:1867-73. 


\section{Chapter}

Summary and general discussion 



\section{Summary and general discussion}

The incidence of type 2 diabetes (T2D), with or without an adverse cardiovascular risk profile has increased dramatically since decades ${ }^{1,2}$. Importantly, cardiovascular risk not only affects individuals with diabetes, but also individuals in the general population, without diabetes ${ }^{3,4}$. An adverse cardiovascular risk profile and/or having T2D are associated with an increased risk of macrovascular diseases (e.g. myocardial infarction, stroke, and peripheral arterial disease) and diseases which are (partly) of microvascular origin (e.g. heart failure, (lacunar) stroke, depression, cognitive decline, retinopathy, chronic kidney disease, and neuropathy). Evidence indicates that endothelial dysfunction is an important underlying mechanism of both macrovascular ${ }^{5-8}$ and microvascular diseases $^{9-14}$. Endothelial cells line the interior surface of all blood vessels, both in the macrocirculation and microcirculation, and are important in blood vessel physiology. This dissertation focused on determinants of microvascular (endothelial) dysfunction in individuals with and without T2D in order to understand its pathophysiology better.

Macrovascular (endothelial) dysfunction occurs in prediabetes with further deterioration in $\mathrm{T}_{2} \mathrm{D}^{15-17}$. This suggests that the pathogenesis of T2D-associated macrovascular disease starts before diabetes occurs (ticking clock hypothesis ${ }^{18}$ ). However, whether microvascular endothelial dysfunction already occurs in prediabetes has not been investigated extensively ${ }^{17,19}$. Key metabolic features of T2D are insulin resistance and $\beta$-cell dysfunction leading to hyperglycemia and abnormal insulin signaling. In addition, T2D typically develops with an adverse cardiovascular risk profile consisting of low-grade inflammation ${ }^{20}$, dyslipidemia ${ }^{21}$, hypertension ${ }^{22}$, and arterial stiffness $^{16}$. We investigated to what extent prediabetes- and T2D-associated microvascular dysfunction were potentially attributable to these metabolic and vascular risk factors.

In the general population, aging, male sex, dyslipidemia, obesity, hyperglycemia (including prediabetes and T2D), hypertension, current smoking, low levels of physical activity, and high levels of sedentary time are major determinants of macrovascular diseases. These risk factors may act via inducing large-artery endothelial dysfunction ${ }^{8,23}$, atherosclerosis $^{24,25}$, and/or arterial stiffening ${ }^{26-28}$. However, because of endothelial cell heterogeneity (i.e. endothelial cells differ remarkably in function and structure depending on their localization), this does not necessarily imply that microvascular endothelial function is affected similarly ${ }^{29}$. As many of the risk factors of macrovascular diseases are also associated with microvascular diseases ${ }^{30-33}$, we hypothesized that they are also determinants of microvascular endothelial function.

Against this background, we investigated in a population-based cohort study, whether (1) prediabetes and T2D are associated with microvascular endothelial 
dysfunction, and whether (pre)diabetes-associated microvascular dysfunction is potentially attributable to metabolic and/or vascular risk factors. (2) We further investigated associations between (modifiable) cardiovascular risk factors and microvascular endothelial function in the general population. An epidemiological approach, like we used in The Maastricht Study, has multiple advantages over small-scale (intervention) studies; first, it allows assessment of various outcomes and determinants. Second, it enables investigation of relatively unbiased associations (e.g. without (or with proper adjustment for) confounding and without selection bias). Third, it allows translation of results to the source population, and possibly also the general population $^{34}$. In this final chapter the key findings of the dissertation are summarized and discussed in the light of the current literature and methodological considerations.

\section{Summary of the main findings}

Microvascular (endothelial) dysfunction has been shown to be associated with, and may therefore be an important underlying mechanism of common diseases, such as heart failure $^{9}$, (lacunar) stroke ${ }^{10}$, depression ${ }^{11}$, cognitive decline ${ }^{14}$, retinopathy ${ }^{12}$, chronic kidney disease ${ }^{13}$, and neuropathy ${ }^{12}$. These diseases occur in the general population and more frequently in individuals with $\mathrm{T}_{2} \mathrm{D}^{35}$. In this dissertation we focused on microvascular (endothelial) (dys)function, more specifically on the stimulus-induced vasodilation capacity of the retinal arteriolar microcirculation and skin microvasculature, measured by flicker light-induced retinal arteriolar dilation and heat-induced skin hyperemia, respectively. Impairments in stimulus-induced retinal arteriolar dilation and skin hyperemia can be seen as a reflection of microvascular endothelial dysfunction ${ }^{36,37}$, as impairments in both responses depend on decreased bioavailability of (endotheliumderived) vasodilators such as nitric oxide and endothelium-dependent hyperpolarizing factors $^{36-38}$. Both responses are (partly) blunted by inhibition of $\mathrm{N}^{G}$-monomethylL-arginine (L-NMMA); a non-selective inhibitor of nitric oxide synthase ${ }^{36-39}$. Nitric oxide is an important endothelium-derived cellular signaling molecule, which relaxes vascular smooth muscle cells and thereby acts as a potent vasodilator. However, this implies that impairments in retinal and skin microvascular vasodilation responses possibly also depend on vascular smooth muscle cell dysfunction ${ }^{40}$. In addition, these responses may also be caused by neuronal dysfunction, as intact retinal and skin nerve signaling is necessary to sense and conduct the flicker and heat stimulus, respectively ${ }^{37,41}$.

All findings described in this dissertation are based on data from the first 3451 individuals of The Maastricht Study ${ }^{42}$. The Maastricht Study is an ongoing prospective 
population-based cohort study that focuses on the etiology, pathophysiology, complications, and comorbidities of $\mathrm{T}^{2} \mathrm{D}^{42}$.

In chapter 2 we tested and confirmed the hypothesis that microvascular endothelial dysfunction is already present in prediabetes with further deterioration in T2D. The regression coefficient of prediabetes was consistently $\sim 1 / 2$ to $1 / 4$ of the T2D coefficient. The interpretation of a graded decline in microvascular function with worsening glucose tolerance is supported by the significant linear associations of higher levels of glycated hemoglobin $\mathrm{A} 1 \mathrm{c}(\mathrm{HbA} 1 \mathrm{c})$ and fasting plasma glucose with attenuated retinal and skin microvascular responses. Importantly, all associations were independent of major cardiovascular risk factors.

In chapter 3 we investigated, with mediation analyses, whether and to what extent prediabetes- and T2D-associated retinal arteriolar and skin microvascular dysfunction are potentially attributable to key metabolic and vascular features associated with T2D, such as hyperglycemia, insulin resistance, elevated blood pressure, arterial stiffness, dyslipidemia, and low-grade inflammation. We showed that hyperglycemia itself, rather than the cardiovascular risk context associated with (pre)diabetes, is the main contributor to both prediabetes- and T2D-associated retinal and skin microvascular dysfunction. In contrast, in this relatively well-treated population, insulin resistance, blood pressure, arterial stiffness, lipid profile, and low-grade inflammation did not significantly contribute to (pre)diabetes-associated microvascular dysfunction.

From chapter 2 and 3 it follows that microvascular endothelial dysfunction is a feature of both prediabetes and T2D and is mainly attributable to hyperglycemia itself, rather than the cardiovascular risk context in which (pre)diabetes typically develops. This implies an early detrimental effect of hyperglycemia on the retinal and skin microvascular responses. Our findings therefore suggest early and intensive glycemic control in (pre)diabetes as a promising therapeutic target for the prevention of (pre)diabetes-associated microvascular dysfunction.

In chapter 4 we investigated whether determinants of macrovascular dysfunction (aging, male sex, hypertension, dyslipidemia, hyperglycemia, higher waist circumference, and current smoking) were also determinants of microvascular dysfunction. We demonstrated that aging and higher levels of glycemia were inversely associated with retinal and skin microvascular vasodilation. Male sex and current smoking were associated with impaired heat-induced skin hyperemia. 24-h systolic blood pressure, waist circumference, and total-to-HDL cholesterol ratio were not significantly associated with these microvascular functions. Hence, it follows that the pattern of associations between cardiovascular risk factors and retinal and skin microvascular function partly resembles the one between cardiovascular risk factors and macrovascular function. Thus, impairment of microvascular function may constitute a pathway through which an 
adverse cardiovascular risk factor pattern may increase risk of diseases of (partly) microvascular origin.

In chapter 5 we examined associations of the modifiable lifestyle risk factors, little habitual physical activity and sedentary behavior, with skin and retinal microvascular function. In addition, we investigated whether these associations were stronger in individuals with T2D as compared to those without. There were three novel findings. First, higher levels of habitual total and higher-intensity physical activity were independently associated with greater skin microvascular vasodilation in individuals with, but not in those without T2D. Second, habitual sedentary time was not associated with skin microvascular function. Third, habitual physical activity and sedentary behavior were not associated with retinal microvascular function. These findings suggest that increasing habitual daily physical activity levels should be investigated as a means to improve microvascular function, at least in individuals with T2D.

\section{Concluding remarks}

In conclusion, using an epidemiological approach, we showed that prediabetes, T2D, and multiple cardiovascular risk factors (i.e. aging, male sex, hyperglycemia, current smoking, and low levels of physical activity) are associated with retinal and/or skin microvascular endothelial dysfunction. Importantly, all associations were independent of major cardiovascular risk factors and were based on data derived from a population-based study, which expanded the evidence of the associations as found in small studies with selected patient groups ${ }^{43-48}$ to the general population. As all our conclusions were based on cross-sectional data, longitudinal studies are needed to further investigate temporality. Longitudinal studies should elucidate whether adverse cardiometabolic risk factors increase the risk of microvascular diseases via impairments in microvascular endothelial function. From a clinical point of view, it is of great importance to target individual risk factors, or clusters of risk factors, in order to reduce microvascular endothelial dysfunction. The ultimate goal is to reduce and/or prevent common diseases of microvascular origin such as heart failure, (lacunar) stroke, depression, cognitive decline, retinopathy, chronic kidney disease, and neuropathy. 


\section{Main findings and their interpretation}

\section{Hyperglycemia is the main contributor to prediabetes- and type 2 diabetes-associated retinal and skin microvascular endothelial dysfunction}

From chapter 2 it follows that microvascular endothelial dysfunction is a feature of both prediabetes and $T 2 \mathrm{D}^{49,50}$, which was supported by linear associations of higher levels of $\mathrm{HbA1C}$ and fasting plasma glucose with attenuated retinal and skin microvascular responses. These findings are in line with earlier insufficiently-adjusted studies, in which small numbers of highly selected individuals were studied ${ }^{19,51,52}$. In contrast to those studies, we expanded the validity of the findings to a population-based level. Importantly, all associations were independent of a broad array of major cardiovascular risk factors.

We earlier showed significant interaction between on the one hand prediabetes and T2D and on the other large-artery endothelial dysfunction with regard to the risk of macrovascular diseases ${ }^{53}$. This implies that individuals with prediabetes and T2D, as compared to those without, are more vulnerable to the detrimental effects of large-artery endothelial dysfunction in the development of cardiovascular disease ${ }^{54}$. Interactions may also be present between on the one hand prediabetes and T2D and on the other microvascular endothelial dysfunction with regard to the risk of microvascular diseases. Microvascular endothelial dysfunction has been associated with, and thus may contribute to the development of these common diseases of microvascular origin ${ }^{9-14}$. Indeed it has been shown that both microvascular endothelial dysfunction and microvascular diseases occur more frequently in individuals with prediabetes and T2D than in those without ${ }^{31,35,49}$. Thus, our findings support the concept that microvascular endothelial dysfunction precedes the clinical diagnosis of T2D and may contribute to the development of microvascular diseases in prediabetes and $\mathrm{T}^{3} \mathrm{D}^{35}$.

In chapter 3 we demonstrated that prediabetes- and T2D-associated retinal arteriolar and skin microvascular dysfunction are mainly attributable to hyperglycemia. Hyperglycemia may impair microvascular function, via the formation of advanced glycation end products and/or reactive oxygen species, both of which can lead to impaired nitric oxide bioavailability, by quenching endothelium-derived nitric oxide and directly inhibit nitric oxide synthase activity ${ }^{55-60}$. However, a vicious circle may exist between hyperglycemia and microvascular endothelial function ${ }^{61}$, as microvascular endothelial function can cause hyperglycemia by impairing insulin secretion ${ }^{62}$ and/or by impairing the timely access of glucose and insulin to their target tissue ${ }^{63}$.

In contrast, indices of insulin resistance, blood pressure, arterial stiffness, lipid profile, and low-grade inflammation did not significantly contribute to (pre)diabetes- 
associated microvascular dysfunction. These findings were (partly) unexpected, and for reasons explained below, need to be interpreted with caution. There is evidence that each of these comorbid vascular risk factors is also linked to microvascular dysfunction, through adverse effects on the insulin signaling pathway, impairment of nitric oxide bioavailability, abnormal regulation of vasomotor tone, and/or increased detrimental pulsatile flow ${ }^{45,61,64-66}$.

First, the homeostatic model assessment (HOMA2)-index we used to assess insulin resistance may differ pathophysiologically from the hyperinsulinemic-euglycemic clamp, which is the gold standard ${ }^{67}$. Clamp-derived insulin resistance mainly measures insulinmediated glucose disposal in peripheral tissue such as muscle ${ }^{68}$, whereas HOMA2 better reflects hepatic insulin resistance ${ }^{69}$. The HOMA2-index we used was likely to be less accurate than for instance the index of hyperglycemia (based on the oral glucose tolerance test (OGTT)) and blood pressure (based on ambulatory 24-h blood pressure measures). Second, the frequent use of antihypertensive and lipid-modifying medication in individuals with prediabetes and T2D, likely caused the small differences in actual blood pressure and lipid profile between individuals without and with T2D. These small differences may have limited the possibility to assess a mediating effect of actual blood pressure and/or lipid profile on (pre)diabetes-associated microvascular endothelial dysfunction. Indeed, the use of antihypertensive medication partly contributed to microvascular endothelial dysfunction in (pre)diabetes, suggesting that previous exposure to elevated blood pressure, rather than actual blood pressure may be important. Third, the absence of a significant contribution of arterial stiffness on, especially, (pre)diabetes-associated retinal microvascular dysfunction was somewhat unexpected, as the retina, as low-impedance tissue, is known to be sensitive to the higher flow pulsatility associated with arterial stiffnening ${ }^{70}$. Possibly, the difference in arterial stiffness between individuals without and with (pre)diabetes was too small in this relatively well-treated population ${ }^{71}$. Last, inflammatory markers drawn from venous plasma, as compared to local measurement, may have underestimated the contribution of the low-grade inflammation index on (pre)diabetes-associated microvascular endothelial dysfunction ${ }^{72}$.

From chapter 2 and 3 it follows that hyperglycemia itself, rather than the cardiovascular risk context in which diabetes typically develops, is the main contributor to the differences in retinal and skin microvascular function between individuals with prediabetes or relatively well-controlled T2D and individuals with normal glucose metabolism (NGM). These findings suggest early and intensive glycemic control in (pre)diabetes as a promising therapeutic target for the prevention of (pre)diabetesassociated microvascular endothelial dysfunction, with the final goal to reduce and/or prevent microvascular diseases. 


\section{(Modifiable) cardiovascular risk factors as determinants of retinal arteriolar and skin microvascular endothelial function}

Microvascular diseases not only occur in individuals with T2D, but are also common in the general population. It is therefore important to unravel determinants of microvascular endothelial dysfunction in the general population. As a consequence of endothelial cell heterogeneity (i.e. diversity between endothelial cells depending on their localization ${ }^{29}$ ) determinants of macrovascular endothelial dysfunction in the general population, such as aging, male sex, hypertension, dyslipidemia, hyperglycemia, higher waist circumference, and current smoking do not necessarily also have to be determinants of microvascular endothelial dysfunction. In chapter 4 we investigated whether determinants of macrovascular endothelial dysfunction were also determinants of microvascular endothelial dysfunction. We demonstrated that aging and higher levels of glycemia were inversely associated with retinal and skin microvascular vasodilation. Male sex and current smoking were associated with impaired heat-induced skin hyperemia. 24-h systolic blood pressure, waist circumference, and total-to-HDL cholesterol ratio were not significantly associated with these microvascular functions.

As The Maastricht Study by design oversampled individuals with T2D, and it is known that individuals with $\mathrm{T} 2 \mathrm{D}$ have an adverse cardiovascular risk factor profile, we investigated, with interaction analyses, whether associations between cardiovascular risk factors and microvascular endothelial function were driven by the oversampling of individuals with T2D. We found non-significant interactions between any cardiovascular risk factor and T2D with regard to retinal and skin microvascular function. This implies that the associations observed in this T2D-enriched population can be considered valid for a non-oversampled population, i.e. the general population ${ }^{73}$. For our findings this means that aging and higher levels of glycemia, along its entire range, are associated with impaired retinal and skin microvascular function, and that these associations were not driven by the T2D oversampling. This again supports a detrimental effect of early hyperglycemia on microvascular function, which is in line with the conclusions in chapters 2 and 3 .

Current smoking was associated with heat-induced skin hyperemia, but not with retinal arteriolar microvascular function, which is in line with earlier studies ${ }^{46,74}$. Mechanistically, smoking may induce microvascular dysfunction via increased formation of reactive oxygen species and/or inhibition of nitric oxide synthase activity ${ }^{75}$. The absence of an association between smoking and retinal arteriolar microvascular dysfunction was likely to be explained by detrimental effects of smoking on the 'smaller' microvasculature (such as those involved in heat-induced skin hyperemia) and not on the relatively large retinal arterioles we measured ${ }^{74}$. This was supported by the observation from others, that smoking was indeed associated with impaired vasodilation 
of relatively large retinal venules ${ }^{74}$. This suggests that dysfunction of small retinal arterioles and capillaries, leads to impaired downstream blood flow and thereby further hampers the relatively passive venous vasodilation ${ }^{74}$.

The absence of associations of 24-h systolic blood pressure and total-to-HDL cholesterol ratio with retinal and skin microvascular function was unexpected, as earlier small studies have shown microvascular dysfunction to be associated with elevated blood pressure $^{19}$ and hypercholesterolemia ${ }^{76}$. A potential explanation is that in this relatively healthy and well-treated population, the blood pressure and total-to-HDL cholesterol ratio range was insufficiently broad for such associations to appear. In The Maastricht Study, approximately $40 \%$ and $37 \%$ of the individuals used antihypertensive and lipid-modifying medication, respectively. In particular the use of angiotensinconverting-enzyme (ACE)-inhibitors may also directly have improved microvascular endothelial function ${ }^{77}$.

The absence of an inverse association of higher waist circumference and/or body mass index with retinal and skin microvascular function was unexpected ${ }^{43,63}$. Apparently, waist circumference and body mass index do not affect microvascular function as assessed here. However, earlier reports have shown that microvascular vasomotion ${ }^{78}$, post-occlusive reactive hyperemia ${ }^{79}$, and insulin-mediated vasodilation ${ }^{63}$ are indeed impaired in obese individuals. Mechanistically, higher waist circumference and/or body mass index may induce microvascular dysfunction systemically, via an increase in circulating adipose tissue-derived factors, such as tumor necrosis factor- $\alpha$ and free fatty acids, and/or a decrease in the anti-inflammatory adipokine adiponectin ${ }^{63}$, which may consequently impair insulin-mediated vasodilation ${ }^{63}$. In addition, adipokines released by local fat deposits next to the microvasculature (i.e. perivascular adipose tissue), may locally and directly inhibit vasodilatory pathways ${ }^{80}$.

From chapter 4 it follows that the pattern of associations between cardiovascular risk factors and retinal and skin microvascular function partly resembles the one between cardiovascular risk factors and macrovascular function. Thus, impairment of microvascular function may constitute a pathway through which an adverse cardiovascular risk factor pattern may increase risk of diseases of (partly) microvascular origin.

In chapter 5 we examined associations of the modifiable cardiovascular risk factors; levels of habitual physical activity and levels of sedentary time with skin and retinal microvascular function. We hypothesized that these associations were stronger in individuals with T2D as compared to those without, as hyperglycemia is a potent inducer of impaired nitric oxide bioavailability ${ }^{81}$ and microvascular endothelial dysfunction ${ }^{49}$.

There were three novel findings. First, higher levels of habitual total and higherintensity physical activity were independently associated with greater skin microvascular 
vasodilation in individuals with, but not in those without T2D. These findings are in line with earlier smaller studies ${ }^{82,83}$, and are likely to be explained by beneficial effects of physical activity on (hyperglycemia-induced) impaired nitric oxide bioavailability ${ }^{84}$. In individuals without T2D, physical activity-induced improvement in skin microvascular function may only be observed at higher intensities and longer duration of physical activity than those in our study ${ }^{85,86}$, which explains the absence of an association in these individuals. Second, habitual sedentary time was not associated with skin microvascular function. This may be explained by frequent transient interruptions of habitual sedentary behavior by walking and/or standing, in such a way that detrimental effects did not appear ${ }^{87,88}$. Such interruptions have also been shown to be beneficial for cardiovascular risk ${ }^{89}$. Third, habitual physical activity and sedentary behavior were not associated with retinal microvascular function. This may be explained by autoregulation of retinal blood flow, which during physical activity preserves stable retinal perfusion ${ }^{90,91}$, and consequently does not increase shear stress, in order to maintain visual acuity. In conclusion, results from chapter 5 suggest that increasing habitual daily physical activity should be investigated as a means to improve microvascular function at least in individuals with T2D.

\section{Methodological considerations and challenges}

The studies presented in this dissertation were based on data of The Maastricht Study, an ongoing prospective population-based cohort study that focuses on the etiology, pathophysiology, complications, and comorbidities of T2D. The results need to be interpreted in light of its methodological limitations. Internal and external validity as well as causal inference will be discussed.

\section{Internal validity}

Internal validity refers to the approximate truth about inferences regarding the source population $^{34}$. In cohort studies, confounding, overadjustment bias, information bias, and selection bias may be potential threats to the internal validity.

\section{Confounding and overadjustment bias}

Confounding occurs when a third variable affects the association between the variable of interest and outcome ${ }^{92}$. To minimize the possibility of confounding, an extensive phenotyping approach of individuals of The Maastricht Study was used, which enabled us to correct for various potential confounders in the regression models. However, in 
observational studies, it is impossible to fully exclude the possibility of residual confounding, for instance caused by errors in the measurement of confounders and/or unmeasured confounders ${ }^{34}$. For example, the set of confounders we used may not truly reflect cumulative lifetime exposure. In addition, data on dietary habits, which may affect microvascular endothelial function ${ }^{93,94}$, were not yet available. To test the robustness of our findings, we conducted several sensitivity analyses, with extra confounders and/or substitution of confounders in the regression models. All these sensitivity analyses gave qualitatively similar results and only marginally changed regression coefficients when compared to the main regression model. This makes it unlikely that residual confounding has influenced the associations reported.

As a consequence of the adjustment for multiple confounders, overadjustment bias ${ }^{95}$ may have occurred. Overadjustment bias leads to underestimation of associations and may occur when analyses are adjusted for a proposed confounder that could also act as 1) an intermediate (between determinant and outcome) and/or 2) a proximal causal factor (a factor that lies in the causal pathway prior to the determinant), and/or 3) a proxy of the outcome ${ }^{95}$. To minimize the effects of overadjustment bias, we constructed different regression models. These were based on covariates grouped according to their putative roles, and we examined changes in regression coefficients after sequentially adding these groups of variables to the regression models. The regression models in which we adjusted for (diabetic) retinopathy, nephropathy, and history of cardiovascular disease are likely overadjusted as those diseases have a (micro)vascular origin. Results from these models should therefore be interpreted conservatively.

\section{Measurement and information bias}

Errors in the measurement of determinants and outcomes (measurement bias) may result in biased associations between determinants and outcomes (information bias) ${ }^{34}$. In this section we will discuss the validity of the primary outcome variables (flicker lightinduced retinal arteriolar dilation and heat-induced skin hyperemia) and (modifiable) cardiovascular risk factors as determinants of retinal and skin microvascular endothelial function.

Flicker light-induced retinal arteriolar dilation and heat-induced skin hyperemia were used as direct reproducible ${ }^{96,97}$ estimates of microvascular endothelial function. Measurement bias may have occurred in two ways. First, impairments in both these responses reflect decreased bioavailability of nitric oxide and are therefore likely a reflection of microvascular endothelial dysfunction ${ }^{36,37}$. However, both responses may also depend on intact neuronal ${ }^{37,41}$ (e.g. for the retina; neurovascular coupling, for the skin; axonal reflexes) and vascular smooth muscle cell functioning ${ }^{40}$. It is therefore important to acknowledge that stimulus-induced retinal arteriolar and skin vasodilation 
may not purely be a reflection of microvascular endothelial function. Second, as a consequence of the deep phenotyping approach of The Maastricht Study, we were not fully able to measure microvascular function under strict standardized conditions (e.g. in a fasting state, after abstention of exercise for a specific period of time, after an extensive acclimatization period prior to the measurement, and/or with measurements performed only at a fixed time of the day (to avoid diurnal influences)). However, to minimize information bias as a consequence of dietary influences on the microcirculation ${ }^{93,94}$, participants were asked to refrain from caffeine consumption and smoking at least 3 hours before the measurement and were only allowed to have a light meal (breakfast and/or lunch), low in fat content, at least 90 minutes before the measurement. In addition, skin measurements were performed in a climate-controlled room $\left(24^{\circ} \mathrm{C}\right)$ after $\sim 10$ minutes acclimatization. To reduce diurnal influences on microvascular function, participants of The Maastricht Study were randomly assigned to morning, afternoon, or evening measurement time slots. In addition, analyses (in chapter 5) were adjusted for the part of the day in which the measurement was performed. This did not change the associations reported.

Another type of measurement bias may occur when data are expressed incorrectly. In this dissertation, flicker light-induced retinal arteriolar dilation and heat-induced skin hyperemia, were generally expressed as percentage increase from baseline retinal arteriolar diameter and skin blood flow, respectively. The widespread use of ratio variables (percentages) in medical research is generally accepted and implemented mainly because of two reasons; first, there is a need for standardization in clinical and epidemiological studies; second, a ratio variable incorporates two variables (baseline value and post-stimulus value) in a single measure that can be used in multivariable linear regression models ${ }^{98}$. However, some studies argue that ratio variables are statistically inefficient as data expressed in percentages do not accurately scale across the range of baseline values ${ }^{98-100}$. As we acknowledge this possible drawback, we additionally analyzed our data when we used flicker light-induced increase (in measurement units) in retinal arteriolar diameter from baseline diameter, or heatinduced increase in skin blood flow (in perfusion units) from skin baseline blood flow, as outcomes, rather than their percentages. All these additional analyses did not materially change our results and conclusions, which highlights the robustness of our findings.

In chapters 2 and 3 we used the OGTT to classify prediabetes and T2D in order to investigate whether microvascular dysfunction is a feature of both prediabetes and T2D. However, the OGTT may misclassify. Therefore it is important to speculate on whether misclassification could have biased our results; specifically, whether misclassification of (untreated) individuals with $\mathrm{T} 2 \mathrm{D}$ as having prediabetes could have caused an overestimation of microvascular dysfunction in prediabetes. Whether misclassification 
has occurred or not, the finding of microvascular dysfunction in prediabetes with deterioration in T2D was supported by significant inverse linear associations between measures of hyperglycemia and retinal and skin microvascular function. In addition, for reasons described below, we consider it likely that our results actually underestimate rather than overestimate microvascular dysfunction in prediabetes.

An OGTT does not have perfect reproducibility and therefore misclassification (here defined as a second OGTT that does not yield the same classification as the first) occurs, and has been quantified in individuals with NGM, prediabetes, and $T 2 \mathrm{D}^{101}$. However, and crucially, misclassification will occur in all directions, of prediabetes as T2D and vice versa; of prediabetes as NGM and vice versa; etcetera. It is important to note that misclassification of T2D as prediabetes will cause overestimation of microvascular dysfunction in prediabetes. However, misclassification of 1) prediabetes as NGM, of 2) NGM as prediabetes, and 3) of prediabetes as T2D will all cause underestimation of microvascular dysfunction in prediabetes. Direct misclassification of NGM as T2D or of T2D as NGM could be neglected ${ }^{101}$. Thus, it follows that the net result of these two opposing types of misclassification is likely to be an underestimation of microvascular dysfunction in prediabetes. The more so because in our study (in contrast to ${ }^{101}$ ) the NGM group is much larger than the prediabetes group; the quantitatively largest misclassification will therefore be of NGM as prediabetes, i.e. a type of misclassification that results in underestimation.

Importantly, the term 'misclassification' may be considered questionable, because the OGTT is the current gold standard and so its results are 'true' by definition. In the example ${ }^{101}$, the second OGTT is considered the gold standard. A better approach would have been to use the mean of both OGTTs as the gold standard, but these calculations were not available to us. Given abovementioned considerations, we conclude that misclassification is likely to have occurred, but will likely have resulted in underestimation (bias towards the null) of microvascular dysfunction in prediabetes and therefore did not affect the conclusions of the studies reported in chapters 2 and 3 of this dissertation.

In chapter 4 we investigated whether major cardiovascular risk factors were determinants of retinal arteriolar and skin microvascular function. Cardiovascular risk factors and microvascular function were measured according to well-accepted methods with state of the art technologies ${ }^{42}$. In order to investigate the robustness of the associations (and minimize information bias) of blood pressure, obesity, hyperglycemia, and smoking with microvascular function, we substituted determinants in the analyses (e.g. waist circumference for body mass index, fasting plasma glucose for $\mathrm{HbA1c}$ or 2-h postload glucose, 24-h systolic blood pressure for 24-h diastolic blood pressure, 24-h mean arterial pressure, or 24-h pulse pressure, and smoking status for pack-years 
of smoking). In particular 24-h ambulatory blood pressure is a very accurate measure of blood pressure, which is not prone to the 'white coat' effect that may occur when measured in the office. In addition, earlier studies have shown that cardiovascular risk was better predicted by 24-h ambulatory blood pressure than office blood pressure ${ }^{102}$.

In chapter 5 we used the activePAL3 ${ }^{\mathrm{TM}}$ accelerometer to objectively measure daily levels of habitual physical activity and sedentary time, which in contrast to self-reported questionnaires are more precise ${ }^{103}$ and not affected by report bias ${ }^{104}$. One important drawback of the accelerometer is that information about the context (e.g. leisure or work-related, individually or in groups) and type (e.g. desk work, driving, or watching television) of physical activity and sedentary behavior is unknown. In addition to the length of time the accelerometer was worn (in this study 24 hours a day), the total number of days it was worn (in this study 8 consecutive days) is also important to outbalance irregularities in physical activity and sedentary behavior levels as a consequence of awareness due to wearing the device. Participants of The Maastricht Study were highly compliant wearing the accelerometer; namely 6.3 valid days, which is longer than required for adequate assessment of sedentary behavior and close to that required for optimal estimation of physical activity ${ }^{105}$.

\section{Selection of the study population}

Associations in a study may differ from those observed in the source population when participants are selectively included (selection bias) ${ }^{34}$. Selection bias can be caused by factors that influence study participation (e.g. self-selection bias) and/or procedures used to selectively include participants (e.g. complete-case analysis) ${ }^{34}$. In The Maastricht Study, self-selection bias may likelier have resulted in underestimations, rather than overestimations of the associations (chapters 2, 3, 4, and 5) and mediation effects reported (chapter 3). As participants in The Maastricht Study were asked if they were able and/or willing to participate in 3 half-day visit rounds, relatively healthy and/or well-treated individuals may consequently have been attracted (i.e. individuals with an unhealthy vascular risk profile and/or longstanding diabetes, were less likely to participate as a consequence of the disease and/or (vascular) complications). Indeed, self-selection bias has earlier been shown to occur in population-based studies, in which participants were healthier than non-responders ${ }^{106,107}$.

A second type of selection bias may have occurred as we used a complete-case analysis approach in which participants with missing data were excluded. In our studies, data were missing in approximately $35-65 \%$ of the participants (mainly due to logistical reasons (e.g. device unavailability or no trained researcher available to perform the measurement)). If missing values caused by this complete-case analysis approach are completely at random, results remain unbiased ${ }^{108}$. In the studies described in this 
dissertation, individuals excluded from the analyses due to missing data on retinal or skin reactivity measurements and/or covariates were highly comparable to individuals included in the study populations with regard to age, sex, and cardiometabolic risk profile. We therefore assume it unlikely that selection bias has affected the associations reported.

\section{Temporality of the associations}

The present dissertation enhanced the understanding of (pre)diabetes-associated microvascular endothelial dysfunction and highlights which cardiovascular risk factors are determinants of, and thus may contribute to, microvascular dysfunction in the general population. However, we should emphasize that data were cross-sectional. In chapter 3, we conducted mediation analyses to investigate whether metabolic and vascular risk factors contribute to (pre)diabetes-associated microvascular dysfunction, in order to carefully investigate causality. However, cross-sectional studies do not formally allow drawing conclusions of direct causality of associations, as associations may be prone to the possibility of reverse causality ${ }^{109}$. Implications about causality should therefore be provided with caution. In chapters 4 and 5 , reverse causality obviously is not an issue for associations of age and sex with microvascular dysfunction. However, reverse causality may be especially relevant for hyperglycemia ${ }^{61}$ (i.e. microvascular dysfunction may lead to impaired timely access of glucose and insulin to their target tissues $^{63}$ as well as impaired insulin secretion ${ }^{62}$ ), hypertension (i.e. microvascular dysfunction may increase peripheral resistance) ${ }^{110}$, dyslipidemia (i.e. microvascular dysfunction may impair action of endothelial-bound lipoprotein lipase, and thereby hamper triglyceride utilization, which may lead to increased plasma triglycerides and reduced $\mathrm{HDL}$-cholesterol) ${ }^{111}$, and physical activity (i.e. microvascular dysfunction may lead to suboptimal delivery of oxygen and nutrients to tissues (e.g. muscle) on demand $^{61}$, and therefore may hamper physical activity). Despite the limitations of crosssectional data, we believe this dissertation is an important first step in understanding the complexity of the pathophysiology of microvascular dysfunction on a population-based level. Longitudinal studies are needed to further clarify the temporality of the associations reported.

\section{External validity}

External validity is the extent in which results of a study can be generalized to other situations and/or to other people (e.g. populations) ${ }^{95}$. The design of The Maastricht Study at least allows generalizability of our findings to middle-aged and older individuals 
of Caucasian origin. However, associations may differ in populations with a different distribution of determinants. Chapter 4 showed hyperglycemia, aging, current smoking, and male sex as determinants of microvascular function on a population-based level. Therefore, our results may not be representative for populations-based studies, in which by design, predominantly males, current smokers, and/or only elderly people were included. In addition, our findings may not necessarily be translatable to other racial or ethnic groups.

\section{Conclusions and future directions}

The overarching aim of this dissertation was to provide more insight into the pathophysiology of microvascular endothelial dysfunction, an important underlying mechanism in common diseases such as heart failure, (lacunar) stroke, cognitive decline, depression, chronic kidney disease, retinopathy, and neuropathy. These diseases occur in the general population and more frequently in individuals with T2D and put an enormous burden on patients, their families, and social health care systems. We therefore explored determinants of microvascular endothelial dysfunction in individuals with and without T2D.

In conclusion, using an epidemiological approach, we showed that prediabetes and $\mathrm{T} 2 \mathrm{D}$ are associated with retinal and skin microvascular endothelial dysfunction. Indeed continuous levels of glycemia below the clinical threshold of T2D were already associated with microvascular dysfunction. Hyperglycemia itself, rather than the cardiovascular risk context associated with (pre)diabetes, is the main contributor to (pre)diabetes-associated impaired retinal and skin microvascular function. In individuals with T2D, higher levels of physical activity were associated with greater skin microvascular endothelial function. These findings suggest that early and intensive treatment of hyperglycemia as well as increasing physical activity should be investigated as a means to improve microvascular function in T2D. These findings may open the discussion on the implementation of screening for prediabetes and/or early treatment of individuals with prediabetes with glucose-lowering medication ${ }^{112}$. However, future research should first focus on how to implement and improve screening programs for chronic (mild) hyperglycemia. In addition, the benefits of screening and early treatment on outcomes, such as reductions in micro- and macrovascular complications, transition from prediabetes to T2D, side-effects, cost-efficiency, and quality of life should be further investigated ${ }^{113}$.

In addition, in the general population, aging and hyperglycemia were associated with retinal and skin microvascular endothelial dysfunction, and male sex and smoking were 
associated with skin microvascular endothelial dysfunction. This implicates that the pattern of associations between cardiovascular risk factors and retinal and skin microvascular function partly resembles the one between cardiovascular risk factors and macrovascular function. Thus, impairment of microvascular function may constitute a pathway through which an adverse cardiovascular risk factor pattern may increase risk of diseases of (partly) microvascular origin. Importantly, all associations were independent of major cardiovascular risk factors and were based on data derived from a population-based study, which expanded the evidence of the associations as found in small studies with selected patient groups to the general population.

As all conclusions in this dissertation were based on cross-sectional data, longitudinal studies are needed to further investigate temporality. The Maastricht Study represents a valuable cohort for the investigation of longitudinal associations between determinants and microvascular endothelial function. Annual follow-up on mortality, morbidity, and disease development is already in progress, and follow-up measurements of determinants and microvascular function will be planned as soon as budget becomes available. A longitudinal design should elucidate whether adverse cardiometabolic risk factors increase the risk of microvascular diseases via impairments in microvascular endothelial function. From a clinical point of view, physicians should be aware of individual risk factors or clustering of risk factors of microvascular endothelial dysfunction in their patients. The effect of strategies which target these risk factors should be investigated with regard to the efficacy of improving microvascular endothelial function. The ultimate goal is to reduce and/or prevent common diseases of microvascular origin such as heart failure, (lacunar) stroke, depression, cognitive decline, retinopathy, chronic kidney disease, and neuropathy. 


\section{References}

1. Ringborg A, Lindgren P, Martinell M, Yin DD, Schon S and Stalhammar J. Prevalence and incidence of Type 2 diabetes and its complications 1996-2003: estimates from a Swedish population-based study. Diabet Med. 2008;25:1178-86.

2. Guariguata L, Whiting DR, Hambleton I, Beagley J, Linnenkamp U and Shaw JE. Global estimates of diabetes prevalence for 2013 and projections for 2035. Diabetes Res Clin Pract. 2014;103:137-49.

3. Bu S, Ruan D, Yang Z, Xing X, Zhao W, Wang N, Xie L and Yang W. Sex-Specific Prevalence of Diabetes and Cardiovascular Risk Factors in the Middle-Aged Population of China: A Subgroup Analysis of the 2007-2008 China National Diabetes and Metabolic Disorders Study. PLoS One. 2015;10:e0139039.

4. Welin L, Adlerberth A, Caidahl K, Eriksson H, Hansson PO, Johansson S, Rosengren A, Svardsudd K, Welin $C$ and Wilhelmsen L. Prevalence of cardiovascular risk factors and the metabolic syndrome in middle-aged men and women in Gothenburg, Sweden. BMC Public Health. 2008;8:403.

5. Poredos $\mathrm{P}$, Besic $\mathrm{H}$ and Jeraj $\mathrm{L}$. Relationship between endothelial function of micro- and macrocirculation in patients with peripheral arterial disease. Vasa. 2017;46:17-22.

6. Besic H, Jeraj L, Spirkoska A, Jezovnik MK and Poredos P. Deterioration of endothelial function of microand macrocirculation in patients with diabetes type 1 and 2. Int Angiol. 2017;36:354-61.

7. Karatzis EN, Ikonomidis I, Vamvakou GD, Papaioannou TG, Protogerou AD, Andreadou I, Voidonikola PT, Karatzi KN, Papamichael CM and Lekakis JP. Long-term prognostic role of flow-mediated dilatation of the brachial artery after acute coronary syndromes without ST elevation. Am J Cardiol. 2006;98:1424-8.

8. Chen SM, Tsai TH, Hang CL, Yip HK, Fang CY, Wu CJ and Guo GB. Endothelial dysfunction in young patients with acute ST-elevation myocardial infarction. Heart Vessels. 2011;26:2-9.

9. Lee JF, Barrett-O'Keefe Z, Garten RS, Nelson AD, Ryan JJ, Nativi JN, Richardson RS and Wray DW. Evidence of microvascular dysfunction in heart failure with preserved ejection fraction. Heart. 2016;102:278-84.

10. Knottnerus IL, Ten Cate H, Lodder J, Kessels F and van Oostenbrugge RJ. Endothelial dysfunction in lacunar stroke: a systematic review. Cerebrovasc Dis. 2009;27:519-26.

11. Santos M, Xekardaki A, Kovari E, Gold G, Bouras C and Giannakopoulos P. Microvascular pathology in late-life depression. J Neurol Sci. 2012;322:46-9.

12. Gupta A and Bhatnagar S. Vasoregression: A Shared Vascular Pathology Underlying Macrovascular And Microvascular Pathologies? OMICS. 2015;19:733-53.

13. Zafrani $L$ and Ince C. Microcirculation in Acute and Chronic Kidney Diseases. Am J Kidney Dis. 2015;66:1083-94.

14. De Silva TM and Faraci FM. Microvascular Dysfunction and Cognitive Impairment. Cell Mol Neurobiol. 2016;36:241-58.

15. Mostaza JM, Lahoz C, Salinero-Fort MA, de Burgos-Lunar C, Laguna F, Estirado E, Garcia-Iglesias F, Gonzalez-Alegre T, Cornejo-Del-Rio V, Sabin C, Lopez S and Group S-. Carotid atherosclerosis severity in relation to glycemic status: a cross-sectional population study. Atherosclerosis. 2015;242:377-82.

16. Schram MT, Henry RM, van Dijk RA, Kostense PJ, Dekker JM, Nijpels G, Heine RJ, Bouter LM, Westerhof N and Stehouwer CD. Increased central artery stiffness in impaired glucose metabolism and type 2 diabetes: the Hoorn Study. Hypertension. 2004;43:176-81.

17. Su Y, Liu XM, Sun YM, Wang YY, Luan Y and Wu Y. Endothelial dysfunction in impaired fasting glycemia, impaired glucose tolerance, and type 2 diabetes mellitus. Am J Cardiol. 2008;102:497-8.

18. Wong MS, Gu K, Heng D, Chew SK, Chew LS and Tai ES. The Singapore impaired glucose tolerance follow-up study: does the ticking clock go backward as well as forward? Diabetes Care. 2003;26:3024-30.

19. Caballero AE, Arora S, Saouaf R, Lim SC, Smakowski P, Park JY, King GL, LoGerfo FW, Horton ES and Veves A. Microvascular and macrovascular reactivity is reduced in subjects at risk for type 2 diabetes. Diabetes. 1999;48:1856-62.

20. Hajer GR, van Haeften TW and Visseren FL. Adipose tissue dysfunction in obesity, diabetes, and vascular diseases. Eur Heart J. 2008;29:2959-71.

21. Anderson RA, Evans ML, Ellis GR, Graham J, Morris K, Jackson SK, Lewis MJ, Rees A and Frenneaux MP. The relationships between post-prandial lipaemia, endothelial function and oxidative stress in healthy individuals and patients with type 2 diabetes. Atherosclerosis. 2001;154:475-83. 
22. Lonati C, Morganti A, Comarella L, Mancia G, Zanchetti A and Group IS. Prevalence of type 2 diabetes among patients with hypertension under the care of 30 Italian clinics of hypertension: results of the (Iper)tensione and (dia)bete study. J Hypertens. 2008;26:1801-8.

23. Witte DR, Westerink J, de Koning EJ, van der Graaf Y, Grobbee DE and Bots ML. Is the association between flow-mediated dilation and cardiovascular risk limited to low-risk populations? J Am Coll Cardiol. 2005;45:1987-93.

24. Selwaness M, Hameeteman R, Van 't Klooster R, Van den Bouwhuijsen Q, Hofman A, Franco OH, Niessen WJ, Klein S, Vernooij MW, Van der Lugt A and Wentzel JJ. Determinants of carotid atherosclerotic plaque burden in a stroke-free population. Atherosclerosis. 2016;255:186-92.

25. Sturlaugsdottir R, Aspelund T, Bjornsdottir G, Sigurdsson S, Thorsson B, Eiriksdottir G and Gudnason V. Prevalence and determinants of carotid plaque in the cross-sectional REFINE-Reykjavik study. BMJ Open. 2016;6:e012457

26. Seidlerova J, Filipovsky J and Dolejsova M. Determinants of aortic stiffening in elderly subjects: results of a nine-year follow-up. Blood Press. 2013;22:173-8.

27. Brunner EJ, Shipley MJ, Ahmadi-Abhari S, Tabak AG, McEniery CM, Wilkinson IB, Marmot MG, Singh-Manoux A and Kivimaki M. Adiposity, obesity, and arterial aging: longitudinal study of aortic stiffness in the Whitehall II cohort. Hypertension. 2015;66:294-300.

28. Mikola H, Pahkala K, Niinikoski H, Ronnemaa T, Viikari JS, Jula A, Juonala M and Raitakari OT. Cardiometabolic Determinants of Carotid and Aortic Distensibility From Childhood to Early Adulthood. Hypertension. 2017;70:452-60.

29. Aird WC. Endothelial cell heterogeneity. Cold Spring Harb Perspect Med. 2012;2:a006429.

30. Fraser-Bell S, Symes R and Vaze A. Hypertensive eye disease: a review. Clin Exp Ophthalmol. 2017;45: 45-53.

31. Buysschaert M, Medina JL, Bergman M, Shah A and Lonier J. Prediabetes and associated disorders. Endocrine. 2015;48:371-93.

32. Wickman $\mathrm{C}$ and Kramer H. Obesity and kidney disease: potential mechanisms. Semin Nephrol. 2013;33:14-22.

33. Parekh A, Smeeth D, Milner Y and Thure S. The Role of Lipid Biomarkers in Major Depression. Healthcare. 2017;5.

34. Rothman KJ, Greenland S and Lash TJ. Modern Epidemiology. Philadelphia: Lippincott Williams and Wilkins. 2008.

35. Stratton IM, Adler AI, Neil HA, Matthews DR, Manley SE, Cull CA, Hadden D, Turner RC and Holman RR. Association of glycaemia with macrovascular and microvascular complications of type 2 diabetes (UKPDS 35): prospective observational study. BMJ. 2000;321:405-12.

36. Dorner GT, Garhofer G, Kiss B, Polska E, Polak K, Riva CE and Schmetterer L. Nitric oxide regulates retinal vascular tone in humans. Am J Physiol Heart Circ Physiol. 2003;285:H631-6.

37. Minson CT, Berry LT and Joyner MJ. Nitric oxide and neurally mediated regulation of skin blood flow during local heating. J App/ Physiol (1985). 2001;91:1619-26.

38. Choi PJ, Brunt VE, Fujii N and Minson CT. New approach to measure cutaneous microvascular function: an improved test of NO-mediated vasodilation by thermal hyperemia. J Appl Physiol (1985). 2014;117:277-83.

39. Kellogg DL, Jr., Liu Y, Kosiba IF and O'Donnell D. Role of nitric oxide in the vascular effects of local warming of the skin in humans. J Appl Physiol (1985). 1999;86:1185-90.

40. Lacolley P, Regnault V, Nicoletti A, Li Z and Michel JB. The vascular smooth muscle cell in arterial pathology: a cell that can take on multiple roles. Cardiovasc Res. 2012;95:194-204.

41. Falsini B, Riva CE and Logean E. Flicker-evoked changes in human optic nerve blood flow: relationship with retinal neural activity. Invest Ophthalmol Vis Sci. 2002;43:2309-16.

42. Schram MT, Sep SJ, van der Kallen CJ, Dagnelie PC, Koster A, Schaper NC, Henry RM and Stehouwer CD. The Maastricht Study: an extensive phenotyping study on determinants of type 2 diabetes, its complications and its comorbidities. Eur J Epidemiol. 2014;29:439-51.

43. de Jongh RT, Serne EH, IJzerman RG, de Vries G and Stehouwer CD. Impaired microvascular function in obesity: implications for obesity-associated microangiopathy, hypertension, and insulin resistance. Circulation. 2004;109:2529-35. 
44. Nagel E, Vilser W and Lanzl I. Age, blood pressure, and vessel diameter as factors influencing the arterial retinal flicker response. Invest Ophthalmol Vis Sci. 2004;45:1486-92.

45. Reimann M, Weiss $\mathrm{N}$ and Ziemssen $\mathrm{T}$. Different responses of the retinal and cutaneous microcirculation to transient dysmetabolic conditions. Atheroscler Suppl. 2015;18:1-7.

46. IJzerman RG, Serne EH, van Weissenbruch MM, de Jongh RT and Stehouwer CD. Cigarette smoking is associated with an acute impairment of microvascular function in humans. Clin Sci. 2003;104: 247-52.

47. IJzerman RG, de Jongh RT, Beijk MA, van Weissenbruch MM, Delemarre-van de Waal HA, Serne EH and Stehouwer $C D$. Individuals at increased coronary heart disease risk are characterized by an impaired microvascular function in skin. Eur J Clin Invest. 2003;33:536-42.

48. Irving RJ, Walker BR, Noon JP, Watt GC, Webb DJ and Shore AC. Microvascular correlates of blood pressure, plasma glucose, and insulin resistance in health. Cardiovasc Res. 2002;53:271-6.

49. Sörensen BM, Houben AJ, Berendschot TT, Schouten JS, Kroon AA, van der Kallen CJ, Henry RM, Koster A, Reesink KD, Dagnelie PC, Schaper NC, Schalkwijk CG, Schram MT and Stehouwer CD. Hyperglycemia Is the Main Mediator of Prediabetes- and Type 2 Diabetes-Associated Impairment of Microvascular Function: The Maastricht Study. Diabetes Care. 2017;40:e103-5.

50. Sörensen BM, Houben AJ, Berendschot TT, Schouten JS, Kroon AA, van der Kallen CJ, Henry RM, Koster A, Sep SJ, Dagnelie PC, Schaper NC, Schram MT and Stehouwer CD. Prediabetes and Type 2 Diabetes Are Associated With Generalized Microvascular Dysfunction: The Maastricht Study. Circulation. 2016;134:1339-52.

51. Garhofer G, Zawinka C, Resch H, Kothy P, Schmetterer L and Dorner GT. Reduced response of retinal vessel diameters to flicker stimulation in patients with diabetes. Br J Ophthalmol. 2004;88:887-91.

52. Lott ME, Slocomb JE, Shivkumar V, Smith B, Quillen D, Gabbay RA, Gardner TW and Bettermann K. Impaired retinal vasodilator responses in prediabetes and type 2 diabetes. Acta Ophthalmol. 2013;91:e462-9.

53. van Sloten TT, Henry RM, Dekker JM, Nijpels G, Unger T, Schram MT and Stehouwer CD. Endothelial dysfunction plays a key role in increasing cardiovascular risk in type 2 diabetes: the Hoorn study. Hypertension. 2014;64:1299-305.

54. de Jager J, Dekker JM, Kooy A, Kostense PJ, Nijpels G, Heine RJ, Bouter LM and Stehouwer CD. Endothelial dysfunction and low-grade inflammation explain much of the excess cardiovascular mortality in individuals with type 2 diabetes: the Hoorn Study. Arterioscler Thromb Vasc Biol. 2006;26:1086-93.

55. Kim JA, Montagnani M, Koh KK and Quon MJ. Reciprocal relationships between insulin resistance and endothelial dysfunction: molecular and pathophysiological mechanisms. Circulation. 2006;113:1888-904.

56. Soro-Paavonen A, Zhang WZ, Venardos K, Coughlan MT, Harris E, Tong DC, Brasacchio D, Paavonen K, Chin-Dusting J, Cooper ME, Kaye D, Thomas MC and Forbes JM. Advanced glycation end-products induce vascular dysfunction via resistance to nitric oxide and suppression of endothelial nitric oxide synthase. J Hypertens. 2010;28:780-8.

57. Clark MG. Impaired microvascular perfusion: a consequence of vascular dysfunction and a potential cause of insulin resistance in muscle. Am J Physiol Endocrinol Metab. 2008;295:E732-50.

58. Verbeke $\mathrm{P}$, Perichon $\mathrm{M}$, Friguet $\mathrm{B}$ and Bakala $\mathrm{H}$. Inhibition of nitric oxide synthase activity by early and advanced glycation end products in cultured rabbit proximal tubular epithelial cells. Biochim Biophys Acta. 2000;1502:481-94.

59. Vallejo S, Angulo J, Peiro C, Cercas E, Sanchez-Ferrer A, Nevado J, Llergo JL, Rodriguez-Manas L and Sanchez-Ferrer CF. Treatment with acarbose may improve endothelial dysfunction in streptozotocininduced diabetic rats. J Cardiovasc Pharmacol. 2000;36:255-62.

60. De Vriese AS, Verbeuren TJ, Van de Voorde J, Lameire NH and Vanhoutte PM. Endothelial dysfunction in diabetes. BrJ Pharmacol. 2000;130:963-74.

61. Muris DM, Houben AJ, Schram MT and Stehouwer CD. Microvascular dysfunction: an emerging pathway in the pathogenesis of obesity-related insulin resistance. Rev Endocr Metab Disord. 2013;14:29-38.

62. Hashimoto S, Kubota N, Sato H, Sasaki M, Takamoto I, Kubota T, Nakaya K, Noda M, Ueki K and Kadowaki T. Insulin receptor substrate-2 (Irs2) in endothelial cells plays a crucial role in insulin secretion. Diabetes. 2015;64:876-86.

63. Jonk AM, Houben AJ, de Jongh RT, Serne EH, Schaper NC and Stehouwer CD. Microvascular dysfunction in obesity: a potential mechanism in the pathogenesis of obesity-associated insulin resistance and hypertension. Physiology. 2007;22:252-60. 
64. Mitchell GF, Vita JA, Larson MG, Parise H, Keyes MJ, Warner E, Vasan RS, Levy D and Benjamin EJ. Cross-sectional relations of peripheral microvascular function, cardiovascular disease risk factors, and aortic stiffness: the Framingham Heart Study. Circulation. 2005;112:3722-8.

65. Shore AC and Tooke JE. Microvascular function in human essential hypertension. J Hypertens. 1994;12:717-28.

66. Cheng C and Daskalakis C. Association of Adipokines with Insulin Resistance, Microvascular Dysfunction, and Endothelial Dysfunction in Healthy Young Adults. Mediators Inflamm. 2015;2015:594039.

67. Bonora E, Targher G, Alberiche M, Bonadonna RC, Saggiani F, Zenere MB, Monauni T and Muggeo M. Homeostasis model assessment closely mirrors the glucose clamp technique in the assessment of insulin sensitivity: studies in subjects with various degrees of glucose tolerance and insulin sensitivity. Diabetes Care. 2000;23:57-63.

68. Ferrannini E and Mari A. How to measure insulin sensitivity. J Hypertens. 1998;16:895-906.

69. Gutch M, Kumar S, Razi SM, Gupta KK and Gupta A. Assessment of insulin sensitivity/resistance. Indian J Endocrinol Metab. 2015;19:160-4.

70. Cheung N, Sharrett AR, Klein R, Criqui MH, Islam FM, Macura KJ, Cotch MF, Klein BE and Wong TY. Aortic distensibility and retinal arteriolar narrowing: the multi-ethnic study of atherosclerosis. Hypertension. 2007; 50:617-22.

71. Geijselaers SL, Sep SJ, Schram MT, van Boxtel MP, van Sloten TT, Henry RM, Reesink KD, Kroon AA, Koster A, Schaper NC, Dagnelie PC, van der Kallen CJ, Biessels GJ and Stehouwer CD. Carotid stiffness is associated with impairment of cognitive performance in individuals with and without type 2 diabetes. The Maastricht Study. Atherosclerosis. 2016;253:186-93.

72. Koskela UE, Kuusisto SM, Nissinen AE, Savolainen MJ and Liinamaa MJ. High vitreous concentration of IL-6 and IL-8, but not of adhesion molecules in relation to plasma concentrations in proliferative diabetic retinopathy. Ophthalmic Res. 2013;49:108-14.

73. Fitzmaurice $\mathrm{G}$. The meaning and interpretation of interaction. Nutrition. 2000;16:313-4.

74. Garhofer G, Resch H, Sacu S, Weigert G, Schmidl D, Lasta M and Schmetterer L. Effect of regular smoking on flicker induced retinal vasodilatation in healthy subjects. Microvasc Res. 2011;82:351-5.

75. Henriksson P, Lu Q, Diczfalusy $U$ and Freyschuss A. Immediate effect of passive smoking on microcirculatory flow. Microcirculation. 2014;21:587-92.

76. Stapleton PA, Goodwill AG, James ME, Brock RW and Frisbee JC. Hypercholesterolemia and microvascular dysfunction: interventional strategies. J Inflamm. 2010;7:54

77. Galatius S, Wroblewski H, Sorensen V, Haunso S, Norgaard T and Kastrup J. Reversal of peripheral microvascular dysfunction during long-term treatment with the angiotensin-converting enzyme inhibitor fosinopril in congestive heart failure. J Card Fail. 1999;5:17-24.

78. Muris DM, Houben AJ, Kroon AA, Henry RM, van der Kallen CJ, Sep SJ, Koster A, Dagnelie PC, Schram MT and Stehouwer CD. Age, waist circumference, and blood pressure are associated with skin microvascular flow motion: the Maastricht Study. J Hypertens. 2014;32:2439-49.

79. Francischetti EA, Tibirica E, da Silva EG, Rodrigues E, Celoria BM and de Abreu VG. Skin capillary density and microvascular reactivity in obese subjects with and without metabolic syndrome. Microvasc Res. 2011;81:325-30.

80. Yudkin JS, Eringa E and Stehouwer CD. "Vasocrine" signalling from perivascular fat: a mechanism linking insulin resistance to vascular disease. Lancet. 2005;365:1817-20.

81. Hamed S, Brenner B and Roguin A. Nitric oxide: a key factor behind the dysfunctionality of endothelial progenitor cells in diabetes mellitus type-2. Cardiovasc Res. 2011;91:9-15.

82. Colberg SR, Parson HK, Holton DR, Nunnold T and Vinik Al. Cutaneous blood flow in type 2 diabetic individuals after an acute bout of maximal exercise. Diabetes Care. 2003;26:1883-8.

83. Colberg SR, Stansberry KB, McNitt PM and Vinik Al. Chronic exercise is associated with enhanced cutaneous blood flow in type 2 diabetes. J Diabetes Complications. 2002;16:139-45.

84. Nyberg M, Blackwell JR, Damsgaard R, Jones AM, Hellsten $Y$ and Mortensen SP. Lifelong physical activity prevents an age-related reduction in arterial and skeletal muscle nitric oxide bioavailability in humans. J Physiol. 2012;590:5361-70.

85. Tew GA, George KP, Cable NT and Hodges GJ. Endurance exercise training enhances cutaneous microvascular reactivity in post-menopausal women. Microvasc Res. 2012;83:223-8. 
86. Roche DM, Rowland TW, Garrard M, Marwood S and Unnithan VB. Skin microvascular reactivity in trained adolescents. Eur J Appl Physiol. 2010;108:1201-8.

87. Restaino RM, Holwerda SW, Credeur DP, Fadel PJ and Padilla J. Impact of prolonged sitting on lower and upper limb micro- and macrovascular dilator function. Exp Physiol. 2015;100:829-38.

88. Demiot C, Dignat-George F, Fortrat JO, Sabatier F, Gharib C, Larina I, Gauquelin-Koch G, Hughson R and Custaud MA. WISE 2005: chronic bed rest impairs microcirculatory endothelium in women. Am J Physiol Heart Circ Physiol. 2007;293:H3159-64.

89. Benatti FB and Ried-Larsen M. The Effects of Breaking up Prolonged Sitting Time: A Review of Experimental Studies. Med Sci Sports Exerc. 2015;47:2053-61.

90. Hayashi N, Ikemura T and Someya N. Effects of dynamic exercise and its intensity on ocular blood flow in humans. Eur J Appl Physiol. 2011;111:2601-6.

91. Risner D, Ehrlich R, Kheradiya NS, Siesky B, McCranor L and Harris A. Effects of exercise on intraocular pressure and ocular blood flow: a review. J Glaucoma. 2009;18:429-36.

92. Grimes DA and Schulz KF. Bias and causal associations in observational research. Lancet. 2002;359: 248-52.

93. Aoqui C, Chmielewski S, Scherer E, Eissler R, Sollinger D, Heid I, Braren R, Schmaderer C, Megens RT, Weber C, Heemann U, Tschop M and Baumann M. Microvascular dysfunction in the course of metabolic syndrome induced by high-fat diet. Cardiovasc Diabetol. 2014;13:31.

94. Jonk AM, Houben AJ, Schaper NC, de Leeuw PW, Serne EH, Smulders YM and Stehouwer CD. Mealrelated increases in microvascular vasomotion are impaired in obese individuals: a potential mechanism in the pathogenesis of obesity-related insulin resistance. Diabetes Care. 2011;34 Suppl 2:S342-8.

95. Schisterman EF, Cole SR and Platt RW. Overadjustment bias and unnecessary adjustment in epidemiologic studies. Epidemiology. 2009;20:488-95.

96. Agarwal SC, Allen J, Murray A and Purcell IF. Comparative reproducibility of dermal microvascular blood flow changes in response to acetylcholine iontophoresis, hyperthermia and reactive hyperaemia. Physiol Meas. 2010;31:1-11.

97. Nguyen TT, Kreis AJ, Kawasaki R, Wang JJ, Seifert BU, Vilser W, Nagel E and Wong TY. Reproducibility of the retinal vascular response to flicker light in Asians. Curr Eye Res. 2009;34:1082-8.

98. Veglia F, Amato M, Giovannardi M, Ravani A, Tedesco CC, Frigerio B, Sansaro D, Tremoli E and Baldassarre D. Potentially spurious correlations between arterial size, flow-mediated dilation, and shear rate. Hypertension. 2014;64:1328-33.

99. Atkinson $\mathrm{G}$ and Batterham AM. The percentage flow-mediated dilation index: a large-sample investigation of its appropriateness, potential for bias and causal nexus in vascular medicine. Vasc Med. 2013;18:354-65.

100. Vickers AJ. The use of percentage change from baseline as an outcome in a controlled trial is statistically inefficient: a simulation study. BMC Med Res Methodol. 2001;1:6.

101. Mooy JM, Grootenhuis PA, de Vries H, Kostense PJ, Popp-Snijders C, Bouter LM and Heine RJ. Intra-individual variation of glucose, specific insulin and proinsulin concentrations measured by two oral glucose tolerance tests in a general Caucasian population: the Hoorn Study. Diabetologia. 1996;39: 298-305.

102. Staessen JA, Thijs L, Fagard R, O'Brien ET, Clement D, de Leeuw PW, Mancia G, Nachev C, Palatini P, Parati G, Tuomilehto J and Webster J. Predicting cardiovascular risk using conventional vs ambulatory blood pressure in older patients with systolic hypertension. Systolic Hypertension in Europe Trial Investigators. JAMA. 1999;282:539-46.

103. Downs A, Van Hoomissen J, Lafrenz A and Julka DL. Accelerometer-measured versus self-reported physical activity in college students: implications for research and practice. I Am Coll Health. 2014;62:204-12.

104. Atkin AJ, Gorely T, Clemes SA, Yates T, Edwardson C, Brage S, Salmon J, Marshall SJ and Biddle SJ. Methods of Measurement in epidemiology: sedentary Behaviour. Int J Epidemiol. 2012;41:1460-71.

105. Barreira TV, Hamilton MT, Craft LL, Gapstur SM, Siddique J and Zderic TW. Intra-individual and interindividual variability in daily sitting time and MVPA. J Sci Med Sport. 2016;19:476-81.

106. Janzon L, Hanson BS, Isacsson SO, Lindell SE and Steen B. Factors influencing participation in health surveys. Results from prospective population study 'Men born in 1914' in Malmo, Sweden. J Epidemiol Community Health. 1986;40:174-7. 
Chapter 6

107. Jacobsen BK and Thelle DS. The Tromso Heart Study: responders and non-responders to a health questionnaire, do they differ? Scand J Soc Med. 1988;16:101-4.

108. Sterne JA, White IR, Carlin JB, Spratt M, Royston P, Kenward MG, Wood AM and Carpenter JR. Multiple imputation for missing data in epidemiological and clinical research: potential and pitfalls. BMJ. 2009;338:b2393.

109. Cole DA and Maxwell SE. Testing mediational models with longitudinal data: questions and tips in the use of structural equation modeling. J Abnorm Psychol. 2003;112:558-77.

110. Karaca U, Schram MT, Houben AJ, Muris DM and Stehouwer CD. Microvascular dysfunction as a link between obesity, insulin resistance and hypertension. Diabetes Res Clin Pract. 2014;103:382-7.

111. Olivecrona G. Role of lipoprotein lipase in lipid metabolism. Curr Opin Lipidol. 2016;27:233-41.

112. Mukhopadhyay P and Chowdhury S. Drug therapy in prediabetes. J Indian Med Assoc. 2005;103:603-8.

113. Whitley HP, Hanson C and Parton JM. Systematic Diabetes Screening Using Point-of-Care HbA1c Testing Facilitates Identification of Prediabetes. Ann Fam Med. 2017;15:162-64. 
Nederlandstalige samenvatting 



\section{Nederlandstalige samenvatting}

Type 2 diabetes (T2D) is een chronische stofwisselingsziekte gekenmerkt door verhoogde glucosewaarden in het bloed. Deze aandoening komt voor bij ruim 1,2 miljoen Nederlanders. Daarnaast heeft ook een groot aantal mensen diabetes, of een voorstadium van diabetes dat prediabetes wordt genoemd, zonder het te weten. Van prediabetes is sprake wanneer de glucosewaarden licht verhoogd zijn maar nog niet zo hoog dat het T2D genoemd wordt. Verhoogde glucosewaarden kunnen leiden tot chronische schade aan zenuwen (neuropathie), ogen (retinopathie) en nieren (nefropathie), de zogenoemde microvasculaire ziekten. Andere veelvoorkomende ziekten die een deels microvasculaire oorzaak hebben zijn hartfalen, (lacunaire) beroerte, depressie en cognitieve achteruitgang. Verhoogde glucosewaarden kunnen ook leiden tot ziekten aan de grote vaten, de zogenoemde macrovasculaire ziekten, zoals een hartinfarct, perifeer arterieel vaatlijden en een beroerte. Naast de verhoogde bloedglucosewaarden gaat T2D, maar ook prediabetes, meestal gepaard met een ongunstig cardiovasculair risicoprofiel bestaande uit bijvoorbeeld hypertensie, overgewicht, laaggradige inflammatie en dislipidemie. Deze risicofactoren verhogen de kans op het krijgen van macrovasculaire en microvasculaire ziekten. Een ongunstig cardiovasculair risicoprofiel kan ook zonder de aanwezigheid van (pre)diabetes leiden tot deze ziekten.

De microcirculatie bestaat uit alle vaten kleiner dan 200-150 $\mu \mathrm{m}$ in diameter en omvat de arteriolen, capillairen en venulen. De microcirculatie speelt een belangrijke rol bij het transport en de uitwisseling van zuurstof, voedingsstoffen en hormonen naar de weefsels. Daarnaast is de microcirculatie belangrijk bij de regulering van de perifere vaatweerstand waardoor grote fluctuaties in hydrostatische druk op het niveau van de capillairen worden voorkomen zodat de lokale bloeddruk optimaal is voor uitwisseling. Belangrijk voor deze functies is een goede balans tussen microvasculaire vaatverwijding en vaatvernauwing. De binnenbekleding van de bloedvatwand, bestaande uit endotheelcellen, speelt hierbij een belangrijke rol. Verschillende uit endotheelcellen afkomstige stoffen (bijvoorbeeld stikstofmonoxide en endotheel afkomstig hyperpolariserende factor (EDHF)) zijn vaatverwijders. Endotheline-1, ook afkomstig uit het endotheel, zorgt juist voor vaatvernauwing. Endotheelcellen komen voor in alle bloedvaten, zowel in de macro- als in de microcirculatie. In dit proefschrift onderzochten wij microvasculaire endotheelfunctie, gemeten in het oog en in de huid als respectievelijk flikker licht geïnduceerde retinale arteriolaire vaatverwijding en hitte geïnduceerde huidhyperemie. Beide vaatverwijdingsresponsen zijn afhankelijk van de biobeschikbaarheid van stikstofmonoxide. Een verminderde stimulus geïnduceerde microvasculaire vaatverwijdingsrespons van retina en/of huid werd gedefinieerd 
als microvasculaire endotheeldisfunctie, mogelijk in combinatie met gladde spierceldisfunctie en/of neuronale disfunctie.

Microvasculaire endotheeldisfunctie is een belangrijk onderliggend mechanisme van veelvoorkomende ziekten zoals hartfalen, (lacunaire) beroerte, depressie, cognitieve achteruitgang, retinopathie, chronisch nierfalen en neuropathie. Deze ziekten komen voor in de algemene populatie en vaker bij mensen met (pre)diabetes, en vormen een enorme belasting voor de patiënten, hun families en het zorgsysteem. Een mogelijke aanpak om deze ziekten te voorkomen is het tegengaan van microvasculaire endotheeldisfunctie. Eerst dient bekend te zijn welke (cardiovasculaire) risicofactoren daarmee geassocieerd zijn en daar dus mogelijk aan kunnen bijdragen. In dit proefschrift ligt de focus op het ontrafelen van determinanten van microvasculaire endotheelfunctie bij mensen met en zonder T2D.

Van macrovasculaire endotheeldisfunctie is al langer bekend dat het voorkomt bij mensen met prediabetes en T2D. De aanwezigheid van macrovasculaire disfunctie bij prediabetes kan verklaren waarom mensen met prediabetes, of mensen die nog niet zo lang gediagnosticeerd zijn met T2D, al wel macrovasculaire ziekten kunnen hebben (de zogenoemde "tikkende klok" hypothese). Het is echter onbekend of eenzelfde hypothese ook geldt voor microvasculaire endotheeldisfunctie, wat kan verklaren waarom mensen met prediabetes of mensen die pas net zijn gediagnosticeerd met T2D al wel microvasculaire ziekten kunnen hebben. Belangrijke metabole kenmerken van T2D zijn insulineresistentie en hyperglykemie. Daarbij gaat T2D vaak gepaard met een ongunstig cardiometabool risicoprofiel bestaande uit hypertensie, arteriële vaatstijfheid, dislipidemie en laaggradige inflammatie. We onderzochten of deze metabole en vasculaire risicofactoren bijdragen aan met (pre)diabetes geassocieerde microvasculaire disfunctie.

In de algemene populatie zijn veroudering, het mannelijk geslacht, dislipidemie, hyperglykemie (zoals bij prediabetes en T2D), hoge bloeddruk, verhoogde middelomtrek, roken, weinig fysieke activiteit en veel zitten belangrijke determinanten van macrovasculaire ziekten. Dit kan mogelijk verklaard worden door endotheeldisfunctie in de grote vaten, atherosclerose en/of arteriële vaatstijfheid. Als gevolg van endotheelcel heterogeniteit (het gegeven dat endotheelcellen verschillen in functie en structuur, afhankelijk van hun lokalisatie), betekent dit niet dat microvasculaire endotheelfunctie dezelfde determinanten heeft. Veel van de risicofactoren voor macrovasculaire ziekten zijn echter ook geassocieerd met microvasculaire ziekten. Onze hypothese is dat deze risicofactoren ook determinanten zijn van microvasculaire endotheelfunctie.

In het licht van bovenstaande had dit proefschrift 3 doelstellingen. Ten eerste, meer inzicht krijgen in de aanwezigheid van microvasculaire endotheeldisfunctie bij mensen met prediabetes en T2D (hoofdstuk 2). Vervolgens werd nagegaan of, en welke, 
metabole en vasculaire risicofactoren bijdragen aan met (pre)diabetes geassocieerde microvasculaire endotheeldisfunctie (hoofdstuk 3). Ten tweede, onderzoeken of, en welke, cardiovasculaire risicofactoren determinanten zijn van microvasculaire endotheeldisfunctie (hoofdstuk 4). Ten derde, het bestuderen van dagelijkse fysieke activiteit en zitgedrag als mogelijke beïnvloedbare risicofactoren voor microvasculaire endotheeldisfunctie (hoofdstuk 5).

Alle doelstellingen in dit proefschrift werden onderzocht met gegevens van de eerste 3451 deelnemers van de De Maastricht Studie. Dit is een grootschalig lopend bevolkingsonderzoek naar T2D, de complicaties hiervan en andere vaak met T2D geassocieerde chronische aandoeningen. Het onderzoek wordt uitgevoerd bij personen van 40-75 jaar die woonachtig zijn in de regio Maastricht-Heuvelland. Een epidemiologische benadering, met een grootschalig bevolkingsonderzoek, heeft verschillende voordelen ten opzichte van kleinschalige (interventie) studies. 1) Het geeft de mogelijkheid om verschillende determinanten en uitkomsten te bepalen en te onderzoeken. 2) De kans op bias in de associaties is kleiner (als gevolg van de correctie voor verschillende veronderstelde confounders). 3) De bevindingen kunnen vertaald worden naar tenminste de bronpopulatie en mogelijk ook de algemene bevolking.

In hoofdstuk 2 onderzochten en bevestigden wij de hypothese dat microvasculaire endotheeldisfunctie (van de retinale arteriole en de huid) al aanwezig is bij mensen met prediabetes en verslechtert bij T2D. De regressiecoëfficiënt van prediabetes was consistent $\sim 1 / 2$ tot $1 / 4$ ten opzichte van de regressiecoëfficiënt van mensen met T2D. De interpretatie van deze stapsgewijze afname in microvasculaire endotheelfunctie met verslechtering van glucosetolerantie werd ondersteund door de significante lineaire associaties tussen hogere waarden van hemoglobine A1c en nuchtere bloedglucose met verslechterde microvasculaire functie van retina en huid.

In hoofdstuk 3 onderzochten wij door middel van mediatieanalyse of, en welke, vaak met (pre)diabetes geassocieerde, metabole en vasculaire risicofactoren (zoals hyperglykemie, insulineresistentie, verhoogde bloeddruk, arteriële vaatstijfheid, dislipidemie en laaggradige inflammatie) bijdragen aan met (pre)diabetes geassocieerde microvasculaire endotheeldisfunctie. De resultaten lieten zien dat hyperglykemie zelf het meeste bijdroeg aan zowel met prediabetes als T2D geassocieerde microvasculaire endotheeldisfunctie van retina en huid. In tegenstelling droegen andere ongunstige cardio-vasculaire risicofactoren zoals insulineresistentie, verhoogde bloeddruk, arteriële vaatstijfheid, dislipidemie en laaggradige inflammatie niet bij aan deze disfunctie.

In hoofdstuk 4 onderzochten wij of cardiovasculaire risicofactoren als determinanten van macrovasculaire endotheeldisfunctie, namelijk veroudering, het mannelijk geslacht, dislipidemie, hyperglykemie (zoals bij prediabetes en T2D), hoge bloeddruk, verhoogde middelomtrek en roken ook determinanten zijn van microvasculaire endotheeldisfunctie. 
Veroudering en hyperglykemie waren geassocieerd met zowel microvasculaire disfunctie van retina als huid. Bovendien hadden mannen en huidige rokers een lagere microvasculaire functie in de huid dan vrouwen en niet-rokers. De 24-uurs systolische bloeddruk, middelomtrek en het cholesterolprofiel waren niet significant geassocieerd met deze microvasculaire functiematen. Dit onderzoek toont aan dat cardiovasculaire risicofactoren voor microvasculaire endotheeldisfunctie gedeeltelijk overeenkomen met de determinanten van macrovasculaire endotheeldisfunctie.

In hoofdstuk 5 onderzochten wij de associaties tussen beïnvloedbare risicofactoren, zoals weinig fysieke activiteit en veel zitgedrag, en microvasculaire endotheeldisfunctie van retina en huid. Bovendien onderzochten wij of deze associaties sterker waren bij mensen met T2D vergeleken met mensen zonder T2D. Er waren 3 nieuwe bevindingen. Ten eerste toonden wij aan dat bij mensen met T2D zowel meer totale beweging als meer zware inspanning geassocieerd waren met betere microvasculaire vaatverwijding in de huid. Deze associatie was niet aanwezig in mensen zonder T2D. Ten tweede bleek, bij zowel mensen met als zonder T2D, zitgedrag niet geassocieerd te zijn met microvasculaire endotheeldisfunctie in de huid. Ten derde, en zoals verwacht, waren er geen associaties tussen fysieke activiteit en zitgedrag enerzijds en retinale arteriolaire microvasculaire functie anderzijds. Autoregulatie van de retinadoorbloeding kan hiervoor een verklaring zijn.

\section{Conclusies}

Het overkoepelende doel van dit proefschrift was om meer inzicht te krijgen in de determinanten van microvasculaire endotheeldisfunctie bij mensen met en zonder T2D.

Uit hoofdstuk 2 en 3 volgt dat microvasculaire endotheeldisfunctie zowel voorkomt bij mensen met prediabetes en T2D. (Vroege) hyperglykemie draagt het meest bij aan de met (pre)diabetes geassocieerde microvasculaire disfunctie, in tegenstelling tot andere cardiovasculaire risicofactoren die niet significant bijdroegen. De resultaten uit hoofdstuk 5 toonden aan dat bij mensen met T2D zowel meer totale beweging als meer zware inspanning geassocieerd waren met grotere microvasculaire hyperemie van de huid. Samengenomen suggereren deze resultaten dat bij mensen met T2D een vroege en intensieve hyperglykemische controle evenals meer bewegen aanknopingspunten zijn voor therapeutische strategieën om microvasculaire endotheeldisfunctie te verminderen dan wel tegen te gaan.

In hoofdstuk 4 toonde wij op populatieniveau aan dat verschillende cardiovasculaire risicofactoren zoals veroudering, mannelijk geslacht, hyperglykemie (zoals bij prediabetes en T2D) en roken geassocieerd waren met microvasculaire endotheeldisfunctie van de retinale arteriole en/of huid. Dit onderzoek verruimt het bewijs van cardiovasculaire risicofactoren als determinanten van microvasculaire 
endotheeldisfunctie, zoals gevonden in kleine studies met sterk geselecteerde patiënten groepen, naar de doorsnee bevolking. Microvasculaire disfunctie zou een 'pathway' kunnen vormen waardoor een ongunstig cardiovasculair risicoprofiel de kans op ziekten met een microvasculaire oorzaak verhoogt.

Alle associaties waren gebaseerd op cross-sectionele gegevens en waren gecorrigeerd voor, en dus onafhankelijk van, belangrijke cardiovasculaire risicofactoren (zoals leeftijd, geslacht, bloeddruk, body mass index, het cholesterolgehalte en de voorgeschiedenis van hart- en vaatziekten). Door deze correcties is getracht de kans op rest confounding in de gevonden associaties zo klein mogelijk te houden. Uit crosssectionele gegevens kunnen geen directe uitspraken over oorzaak-gevolg gedaan worden, hiervoor zijn longitudinale onderzoeken nodig. Deze longitudinale onderzoeken zouden ten doel kunnen hebben om te ontrafelen of een ongunstig cardiovasculair profiel, bij mensen met dan wel zonder T2D, een verhoogd risico geeft op microvasculaire ziekten via microvasculaire endotheeldisfunctie. Vanuit een klinisch oogpunt is het doel om individuele en/of clusters van risicofactoren aan te grijpen om zodoende microvasculaire endotheeldisfunctie te voorkomen. Het uiteindelijke doel is de vermindering en/of het voorkomen van veelvoorkomende ziekten met een microvasculaire origine zoals hartfalen, (lacunaire) beroerte, depressie, cognitieve achteruitgang, retinopathie, chronisch nierfalen en neuropathie. 

Valorization addendum 



\section{Valorization addendum}

Knowledge valorization can be defined as the "process of creating value from knowledge, by making knowledge suitable and/or available for societal purposes, and suitable for translation into competitive products, services, processes, and new commercial activities" (adapted definition based on the National Committee Valorization 2011:8). In this addendum we describe how society may benefit from the work conducted in this dissertation.

The results in this dissertation were based on data of the first 3451 participants of The Maastricht Study, an observational prospective population-based cohort study, which is unique for its extensive phenotyping of individuals including a comprehensive characterization of microvascular function ${ }^{1}$. The Maastricht Study is currently one of the world's largest studies on type 2 diabetes (T2D), its complications, and comorbidities and aims to include 8000 participants by the end of 2018. The deep phenotyping approach allowed the investigation of the associations of different (modifiable) cardiovascular risk factors with microvascular function (assessed as flicker light-induced retinal arteriolar dilation and heat-induced skin hyperemia). Through The Maastricht Study several collaborations with (inter)national universities have been established, which further improve the reputation of Maastricht and the university.

This dissertation focused on microvascular (endothelial) dysfunction, an important underlying mechanism in common diseases such as heart failure ${ }^{2}$, (lacunar) stroke ${ }^{3}$, cognitive decline ${ }^{4}$, depression ${ }^{5}$, chronic kidney disease ${ }^{6}$, retinopathy $^{7}$, and neuropathy ${ }^{7}$, which occur in the general population and more frequently in individuals with $T 2 D^{8}$. These microvascular diseases put an enormous burden on patients, their families, and social health care systems. Therefore, it is important to explore determinants of microvascular dysfunction. Although the findings in this dissertation may not lead to societal benefits at first glance, they have increased insight into the pathophysiology of microvascular dysfunction, which adds new fuel to future research initiatives.

The first key finding of this dissertation is that generalized microvascular dysfunction is a feature of both prediabetes (which affects $21 \%$ of the individuals in our study population, with an even higher prevalence in American cohort studies ${ }^{9}$ ) and $T 2 \mathrm{D}^{10}$, and was mainly attributable to hyperglycemia ${ }^{11}$. This indicates that microvascular risk already starts before the onset of T2D, which may consequently explain the increased risk of microvascular diseases in prediabetes and early T2D (ticking clock hypothesis ${ }^{12}$ ). These findings may open the discussion on the implementation of screening for prediabetes and/or early treatment of individuals with prediabetes with glucose-lowering medication $^{13}$. Obviously, attempts to prevent prediabetes, for instance by stimulating a healthy lifestyle, should have the highest priority. According to the Dutch guidelines for 
cardiovascular risk assessment, individuals with mild hypertension and/or dyslipidemia are advised to adopt a healthy lifestyle, and importantly, also qualify for antihypertensive and/or lipid-modifying medication when their cumulative cardiovascular risk is (highly) elevated ${ }^{14}$. Currently no such (low-threshold) guideline for prevention and treatment of early hyperglycemia (including prediabetes) exists, although this may lead to fewer transitions from prediabetes to T2D ${ }^{15}$. Early treatment with metformin may be an option, as it has been shown to improve microvascular function $^{16}$ and is generally well-tolerated ${ }^{15}$. However, future research should first focus on how to implement and improve screening programs for chronic (mild) hyperglycemia. In addition, the benefits of screening and early treatment on outcomes such as reductions in micro- and macrovascular complications, reduction in transition from prediabetes to T2D, side-effects, cost-efficiency, and quality of life should be further investigated $^{17}$.

Another key finding of this dissertation was the identification of cardiovascular risk factors, such as hyperglycemia (as in prediabetes and T2D), aging, male sex, smoking, and low levels of physical activity, as determinants of microvascular (endothelial) dysfunction in the general population. Hence, from a clinical point of view, efficacy of strategies which target these risk factors in order to prevent microvascular dysfunction, and thereby to reduce risk of microvascular diseases, should be examined. Physicians should be aware of individual clustering of these risk factors as well as combinations of them and the concordant increased microvascular risk. In addition, physicians should encourage individuals to adopt a healthy lifestyle by stimulating physical activity and healthy eating as they have been shown to be associated with ameliorated glycemic levels (i.e. consequently may lower the prevalence of T2D) ${ }^{18}$, which may coincide with improved microvascular function ${ }^{19,20}$. Importantly, our results demonstrated that physical activity may especially be beneficial for microvascular function in individuals with T2D. Based on our data, the results are promising, as only 10 extra minutes of higher-intensity physical activity per day are already associated with a $6.3 \%$ greater skin microvascular endothelial function, in individuals with T2D. However, the necessary beneficial amounts of physical activity and possible health effects of breaking up sedentary time on microvascular disease outcome deserve further investigation before physical activity healthcare guidelines can be revised. In addition, early treatment of elevated blood pressure and dyslipidemia and stimulation of smoking cessation are likely other valuable targets to prevent or reduce microvascular dysfunction. Besides a role for physicians in helping individuals to adopt a healthy lifestyle, for instance via motivational counselling, population-based strategies to promote healthy living should be expanded; for instance via targeted television campaigns, (school) education, and awareness of product warning labelling (i.e. dissuasive pictures on smoking packages) ${ }^{21}$. 
Although the conclusions of this dissertation are relevant for clinic and society, it should be noted that associations presented here were based on cross-sectional data. Therefore future longitudinal data are eagerly awaited to elucidate the temporality of the associations reported. The Maastricht Study is a valuable cohort study for follow-up measurements. Ideally, both cardiovascular risk factors and microvascular function are measured during follow-up, taking into account an adequate follow-up time for the risk factors to exert an (deleterious) effect on microvascular function. Importantly, annual follow-up questionnaires on morbidity, mortality, and complications have already been introduced in The Maastricht Study and may soon add valuable new information on whether impairment of microvascular function may constitute a pathway through which an adverse cardiovascular risk factor pattern may increase risk of diseases of (partly) microvascular origin.

In conclusion, in this addendum, several research initiatives have been described which can be translated into new PhD projects. The results of this dissertation contribute to a better understanding of which cardiovascular risk factors are associated with microvascular (dys)function on a population-based level. Further longitudinal studies should elucidate temporality of the associations reported. In addition, physicians should be aware of individual risk factors or clustering of risk factors in their patients, motivate them to adopt a healthy lifestyle, and eventually treat prediabetes, in order to reduce the concordant microvascular risk. The ultimate goal is to prevent, and/or reduce the risk of diseases which are partly or wholly of microvascular origin. 


\section{References}

1. Schram MT, Sep SJ, van der Kallen CJ, Dagnelie PC, Koster A, Schaper NC, Henry RM and Stehouwer CD. The Maastricht Study: an extensive phenotyping study on determinants of type 2 diabetes, its complications and its comorbidities. Eur J Epidemiol. 2014;29:439-51.

2. Lee JF, Barrett-O'Keefe Z, Garten RS, Nelson AD, Ryan JJ, Nativi JN, Richardson RS and Wray DW. Evidence of microvascular dysfunction in heart failure with preserved ejection fraction. Heart. 2016;102:278-84.

3. Knottnerus IL, Ten Cate H, Lodder J, Kessels F and van Oostenbrugge RJ. Endothelial dysfunction in lacunar stroke: a systematic review. Cerebrovasc Dis. 2009;27:519-26.

4. De Silva TM and Faraci FM. Microvascular Dysfunction and Cognitive Impairment. Cell Mol Neurobiol. 2016;36:241-58.

5. Santos M, Xekardaki A, Kovari E, Gold G, Bouras C and Giannakopoulos P. Microvascular pathology in late-life depression. J Neurol Sci. 2012;322:46-9.

6. Zafrani L and Ince C. Microcirculation in Acute and Chronic Kidney Diseases. Am J Kidney Dis. 2015;66:1083-94

7. Gupta A and Bhatnagar S. Vasoregression: A Shared Vascular Pathology Underlying Macrovascular And Microvascular Pathologies? OMICS. 2015;19:733-53.

8. Stratton IM, Adler Al, Neil HA, Matthews DR, Manley SE, Cull CA, Hadden D, Turner RC and Holman RR. Association of glycaemia with macrovascular and microvascular complications of type 2 diabetes (UKPDS 35): prospective observational study. BMJ. 2000;321:405-12.

9. Rhee MK, Herrick K, Ziemer DC, Vaccarino V, Weintraub WS, Narayan KM, Kolm P, Twombly JG and Phillips LS. Many Americans have pre-diabetes and should be considered for metformin therapy. Diabetes Care. 2010;33:49-54.

10. Sörensen BM, Houben AJ, Berendschot TT, Schouten JS, Kroon AA, van der Kallen CJ, Henry RM, Koster A, Sep SJ, Dagnelie PC, Schaper NC, Schram MT and Stehouwer CD. Prediabetes and Type 2 Diabetes Are Associated With Generalized Microvascular Dysfunction: The Maastricht Study. Circulation. 2016;134:1339-52.

11. Sörensen BM, Houben AJ, Berendschot TT, Schouten JS, Kroon AA, van der Kallen CJ, Henry RM, Koster A, Reesink KD, Dagnelie PC, Schaper NC, Schalkwijk CG, Schram MT and Stehouwer CD. Hyperglycemia Is the Main Mediator of Prediabetes- and Type 2 Diabetes-Associated Impairment of Microvascular Function: The Maastricht Study. Diabetes Care. 2017;40:e103-5.

12. Wong MS, Gu K, Heng D, Chew SK, Chew LS and Tai ES. The Singapore impaired glucose tolerance followup study: does the ticking clock go backward as well as forward? Diabetes Care. 2003;26:3024-30.

13. Mukhopadhyay P and Chowdhury S. Drug therapy in prediabetes. J Indian Med Assoc. 2005;103:603-8.

14. NHG-standaard: Richtlijn Cardiovasculair risicomanagement M84. Nederlands huisartsen genootschap. 2012.

15. Hostalek U, Gwilt M and Hildemann S. Therapeutic Use of Metformin in Prediabetes and Diabetes Prevention. Drugs. 2015;75:1071-94.

16. Jadhav S, Ferrell W, Greer IA, Petrie JR, Cobbe SM and Sattar N. Effects of metformin on microvascular function and exercise tolerance in women with angina and normal coronary arteries: a randomized, double-blind, placebo-controlled study. J Am Coll Cardiol. 2006;48:956-63.

17. Whitley HP, Hanson C and Parton JM. Systematic Diabetes Screening Using Point-of-Care HbA1c Testing Facilitates Identification of Prediabetes. Ann Fam Med. 2017;15:162-64.

18. Lv J, Yu C, Guo Y, Bian Z, Yang L, Chen Y, Hu X, Hou W, Chen J, Chen Z, Qi L, Li L and on behalf of the China Kadoorie Biobank Collaborative G. Adherence to a healthy lifestyle and the risk of type 2 diabetes in Chinese adults. Int J Epidemiol. 2017;46:1410-20.

19. Karatzi K, Protogerou A, Kesse-Guyot E, Fezeu LK, Carette C, Blacher J, Levy BI, Galan P, Hercberg S and Czernichow S. Associations between dietary patterns and skin microcirculation in healthy subjects. Arterioscler Thromb Vasc Biol. 2014;34:463-9.

20. Wang JS. Effects of exercise training and detraining on cutaneous microvascular function in man: the regulatory role of endothelium-dependent dilation in skin vasculature. Eur J Appl Physiol. 2005;93: 429-34. 
21. Mozaffarian D, Afshin A, Benowitz NL, Bittner V, Daniels SR, Franch HA, Jacobs DR, Jr., Kraus WE, Kris-Etherton PM, Krummel DA, Popkin BM, Whitsel LP and Zakai NA. Population approaches to improve diet, physical activity, and smoking habits: a scientific statement from the American Heart Association. Circulation. 2012;126:1514-63. 

Scientific output 



\section{Scientific output}

\section{Dissertation}

1. Sörensen BM, Houben AJ, Berendschot TT, Schouten JS, Kroon AA, van der Kallen CJ, Henry RM, Koster A, Sep SJ, Dagnelie PC, Schaper NC, Schram MT, and Stehouwer $\mathrm{CD}$. Prediabetes and type 2 diabetes are associated with generalized microvascular dysfunction: The Maastricht Study. Circulation. 2016;134:1339-52.

2. Sörensen BM, Houben AJ, Berendschot TT, Schouten JS, Kroon AA, van der Kallen CJ, Henry RM, Koster A, Reesink KD, Dagnelie PC, Schaper NC, Schalkwijk CG, Schram MT, and Stehouwer CD. Hyperglycemia is the main mediator of prediabetesand type 2 diabetes-associated impairment of microvascular function: The Maastricht Study. Diabetes Care. 2017;40:e103-5.

3. Sörensen BM, Houben AJ, Berendschot TT, Schouten JS, Kroon AA, van der Kallen CJ, Henry RM, Koster A, Dagnelie PC, Schaper NC, Schram MT, and Stehouwer CD. Cardiovascular risk factors as determinants of retinal and skin microvascular function: The Maastricht Study. PLoS One. 2017;12e0187324.

4. Sörensen BM, Houben AJ, Koster A, Berendschot TT, Schouten JS, Kroon AA, van der Kallen CJ, Henry RM, Savelberg HC, van Dongen CJ, Eussen JP, van der Berg JD, Dagnelie PC, Schaper NC, Schram MT, and Stehouwer CD. Higher levels of daily physical activity are associated with better skin microvascular function in type 2 diabetes: The Maastricht Study. Submitted.

\section{Other}

1. Sörensen BM, Houben AJ, Martens RJ, and Stehouwer CD. Response by Sörensen et al to Letters Regarding Article "Prediabetes and type 2 diabetes are associated with generalized microvascular dysfunction: The Maastricht Study". Circulation. 2017; 135:e862-3.

2. Martens RJ, Houben AJ, Kooman JP, Berendschot TT, Dagnelie PC, vd Kallen CJ, Kroon AA, Leunissen KM, vd Sander FM, Schaper NC, Schouten JS, Schram MT, Sep SJ, Sörensen BM, Henry RM, and Stehouwer CD. Microvascular endothelial dysfunction is associated with albuminuria: The Maastricht Study. Submitted. 

Dankwoord 


\section{Dankwoord}

De afgelopen vier jaar was een inspirerende, geweldige en ook intensieve periode. $\mathrm{Na}$ het volgen van de Arts-Klinisch Onderzoeker master, lag het in de lijn der verwachting dat ik een promotietraject zou gaan volgen. Mijn afstudeerwetenschapsstage bij De Maastricht Studie heb ik als heel prettig ervaren. Ik ben mede daarom zeer erkentelijk dat ik de kans en het vertrouwen heb gekregen het onderzoek verder door te kunnen zetten. Ik wil graag iedereen hartelijk bedanken die op wat voor manier dan ook heeft bijgedragen aan de totstandkoming van dit proefschrift. Een aantal personen wil ik hiervoor in het bijzonder bedanken:

Mijn promotieteam, prof. dr. Coen Stehouwer, dr. Miranda Schram en dr. Boy Houben, wat fijn dat jullie mijn team vormden. Ik ben jullie heel dankbaar voor alles!

Beste Coen, in alle proefschriften die onder jouw begeleiding tot stand kwamen staan enkel lovende woorden, ik sluit mij daar volledig bij aan. Van jouw ideeën en glasheldere uitleg heb ik veel geleerd. Jouw punctualiteit werkte voor mij inspirerend. Dat jij mij jouw waardevolle commentaar vrijwel altijd binnen een aantal uren mailde heb ik ontzettend gewaardeerd.

Beste Miranda, wat had ik zonder jouw hulp gemoeten? Altijd stond je voor mij klaar, voor overleg, voor ideeën of voor gewoon even een gesprekje tussendoor. Jij bent een belangrijke factor geweest in het aanleren van gestructureerd methodologisch denken.

Beste Boy, wat was jij een fijne copromotor. Ook bij jou kon ik altijd terecht. Je kwam met verhelderende ideeën en hebt mij veel geleerd over de microcirculatie. Zoveel dat wij, mijn kamergenoten en ik, er zelfs een heel whiteboard over hebben kunnen volschrijven. Ik zal dat bord, dat wij "The Mechanism" noemden, niet snel vergeten.

Leden van de leescommissie, prof. dr. Harry Struijker-Boudier, prof. dr. Harry Crijns, Prof. dr. Matthijs Hesselink, dr. Joline Beulens en dr. Richard IJzerman, hartelijk dank voor de tijd die u heeft besteed aan het beoordelen van mijn proefschrift.

Beste coauteurs, in alfabetische volgorde dr. Tos Berendschot, dr. Julianne van der Berg, prof. dr. Pieter Dagnelie, dr. Martien van Dongen, dr. Simone Eussen, dr. Ronald Henry, dr. Carla van der Kallen, dr. Annemarie Koster, prof. dr. Bram Kroon, dr. Remy Martens, dr. Koen Reesink, prof. dr. Hans Savelberg, prof. dr. Casper Schalkwijk, prof. dr. Nicolaas Schaper, dr. Jan Schouten en dr. Simone Sep, veel dank voor de kritische blikken op de manuscripten.

Richard Koopmans en Coen Stehouwer, ik dank jullie voor de mogelijkheid die mij is gegeven de opleiding tot internist te volgen. Dr. Stijn Konings, ik verheug me erop om bij jullie in het Catharina Ziekenhuis in Eindhoven te komen werken. 
Deelnemers van De Maastricht Studie, bedankt voor uw inzet. Mede promovendi en collega's bij De Maastricht Studie en/of elders in het ziekenhuis of de universiteit, dank voor de gezelligheid, uitjes, (spelletjes)pauzes, wandelingen, gesprekken, etentjes, congressen en noem maar op. Een drietal van hen wil ik persoonlijk bedanken.

Beste Ellis en Elton, met jullie beiden heb ik veel tijd doorgebracht en plezier gehad. Bedankt voor jullie steun, gastvrijheid, etentjes, gezelligheid, spelletjesavonden en El Picu-feestjes.

Beste Marnix, mijn kamergenoot, ik heb jou niet toevallig als paranimf gekozen. Jouw steun en humor, alsmede onze (onnozele) gesprekken en honderden weddenschapjes om 'tientjes' hebben bijgedragen aan een toptijd. Ik kijk ernaar uit in de toekomst weer samen te werken.

Lieve vrienden en (schoon)familie, bedankt voor jullie positivisme, interesse en dat jullie altijd voor mij klaarstaan.

Lieve mama en zusje, wat is het fijn om te weten dat er altijd een basis is waarop ik kan terugvallen. Bedankt voor alle liefde en onvoorwaardelijke steun die jullie mij al mijn hele leven geven. Dat mijn zusje eveneens mijn paranimf is, ontroert mij.

Lieve papa en opa, ik hoop dat jullie op een of andere manier toch hebben kunnen meekrijgen dat ik in jullie voetsporen ben getreden.

Liefste, of een van de vele andere koosnaampjes die we voor elkaar gebruiken, wat ben ik trots dat jij voor mij gekozen hebt. Jouw liefde en steun zijn van onschatbare waarde. 

Curriculum vitae 



\section{Curriculum vitae}

Ben Sörensen was born on September 11, 1987 in Bombay, India. He was raised in the small village of Enspijk, Gelderland, The Netherlands. He graduated from secondary school in 2005 (VWO, O.R.S. Lek \& Linge, Culemborg). In 2006 he started Biomedical Sciences at the University of Amsterdam, where he took his bachelor's degree in 2009. That same year he started the combined medical master (Medicine and Clinical Science; Arts-Klinisch Onderzoeker (A-KO)), at Maastricht University, where he obtained his medical and Master of Science degree in 2013. Subsequently, he started his PhD research under supervision of Prof. dr. Coen Stehouwer, dr. Boy Houben, and dr. Miranda Schram at the Department of Internal Medicine of Maastricht University Medical Centre, within the CARIM School for Cardiovascular Disease. From January 1, 2018, Ben has worked as a resident internal medicine at Catharina Ziekenhuis Eindhoven. 
